\title{
Ag-Catalyzed Asymmetric Interrupted Barton-Zard Reaction Enabling the Enantioselective Dearomatization of 2- and 3-Nitroindoles
}

Wei-Cheng Yuan, ${ }^{* \dagger}{ }^{\dagger}$ Xin-Meng Chen, ${ }^{\ddagger}$ Jian-Qiang Zhao, ${ }^{*}{ }^{\dagger}$ Yan-Ping Zhang, ${ }^{\dagger}$ Zhen-Hua Wang, ${ }^{\dagger}$ and Yong You ${ }^{\dagger}$

'Innovation Research Center of Chiral Drugs, Institute for Advanced Study; School of Pharmacy, Chengdu University, Chengdu, 610106, China

National Engineering Research Center of Chiral Drugs, Chengdu Institute of Organic Chemistry, Chinese Academy of Sciences, Chengdu, 610041, China

\author{
Email: yuanwc@cioc.ac.cn \\ zhaojianqiang@cdu.edu.cn
}

\section{Supporting Information}

\section{Table of Contents}

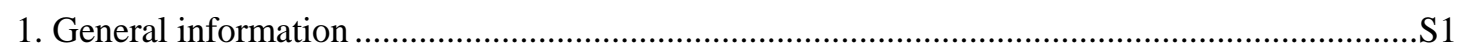

2. General experimental procedures for synthesis of L1-L4 ......................................................... 1

3. General experimental procedures for asymmetric synthesis of compounds 3 ..........................S1

4. Optimization of reaction conditions for the 3-nitroindoles and isocyanoesters ...........................S9

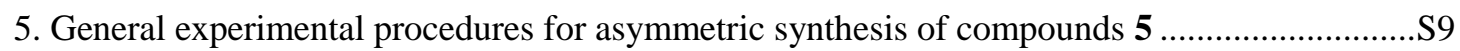

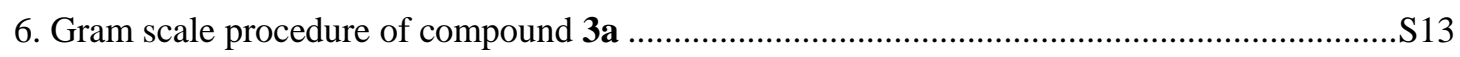

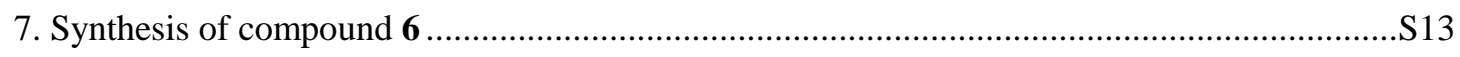

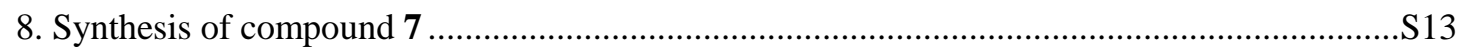

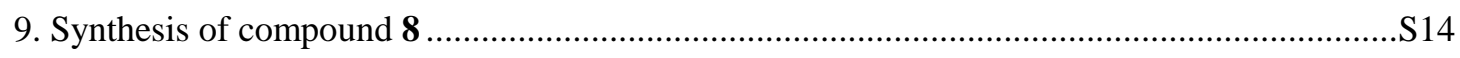

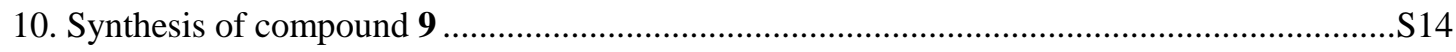

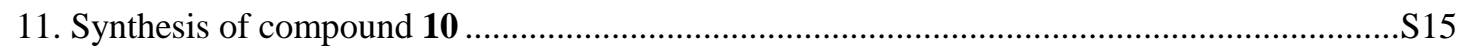

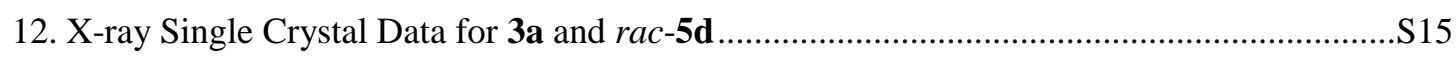

13. Determination of the absolute configuration of $\mathbf{5 d}$ by ECD spectrum..................................... 17

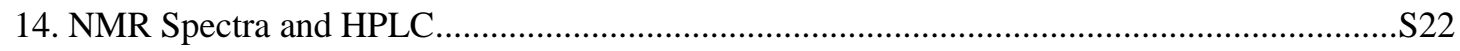




\section{General information}

Reagents were purchased from commercial sources and were used as received unless mentioned otherwise. Reactions were monitored by TLC. ${ }^{1} \mathrm{H}$ NMR and ${ }^{13} \mathrm{C}$ NMR (300 and $75 \mathrm{MHz}$, respectively) spectra were recorded in $\mathrm{CDCl}_{3} .{ }^{1} \mathrm{H}$ NMR chemical shifts are reported in ppm relative to tetramethylsilane (TMS) with the solvent resonance employed as the internal standard $\left(\mathrm{CDCl}_{3}\right.$ at $7.26 \mathrm{ppm}$ ). Data are reported as follows: chemical shift, multiplicity ( $\mathrm{s}=$ singlet, br s = broad singlet, $\mathrm{d}=$ doublet, $\mathrm{t}=$ triplet, $\mathrm{q}=$ quartet, $\mathrm{m}=$ multiplet $)$, coupling constants $(\mathrm{Hz})$ and integration. ${ }^{13} \mathrm{C}$ NMR chemical shifts are reported in ppm from tetramethylsilane (TMS) with the solvent resonance as the internal standard $\left(\mathrm{CDCl}_{3}\right.$ at $77.20 \mathrm{ppm}$,). Melting points were recorded on a melting point apparatus. The enantiomeric excesses were determined by chiral HPLC analysis or ${ }^{1} \mathrm{H}$ NMR. HPLC analysis was performed on Shimadzu SCL-10AVP HPLC systems consisting of the followings: pump, LC-10AD; detector, SPD-10A measured at $254 \mathrm{~nm}$. HRMS was recorded on Bruker Q TOF. Optical rotations were measured with a Perkin-Elmer-341 polarimeter.

2. General experimental procedures for synthesis of L1-L4 (with the synthesis of L3 as example). ${ }^{1}$

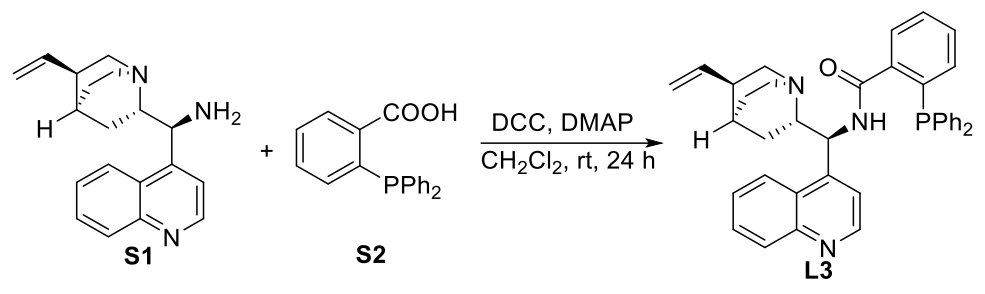

9-Amino epicinchonine $\mathbf{S 1}(1.47 \mathrm{~g}, 5 \mathrm{mmol})$ was dissolved in dry $\mathrm{CH}_{2} \mathrm{Cl}_{2}(25 \mathrm{~mL})$. Then, 2(diphenylphosphino)benzoic acid S2 (1.53, $5 \mathrm{mmol}$ ), 4-dimethylaminopyridine (122 $\mathrm{mg}, 20 \mathrm{~mol} \%$ ) and $N, N^{\prime}$-Dicyclohexylcarbodiimide (1.24 g, 1.2 equiv.) were sequentially added. After stirring for $24 \mathrm{~h}$ at room temperature (TLC monitored), the reaction mixture was concentrated and purified by flash chromatography on silica gel $(\mathrm{EtOAc} / \mathrm{MeOH}=20: 1)$ to give product $\mathbf{L 3}$ as the white solid ( $2.1 \mathrm{~g}, 72 \%$ yield). According to similar method to obtain $\mathbf{L 1}, \mathbf{L 2}$ and $\mathbf{L 4}{ }^{1}$

\section{General experimental procedures for asymmetric synthesis of compounds 3}

To a flame dried reaction tube were added $\mathrm{AgSbF}_{6}(1.7 \mathrm{mg}, 5 \mathrm{~mol} \%)$ and chiral ligand $\mathbf{L 3}(2.8$ $\mathrm{mg}, 6 \mathrm{~mol} \%)$, followed by addition distilled toluene $(1.0 \mathrm{~mL})$. The reaction solution was stirred at room temperature for $1 \mathrm{~h}$ under an argon atmosphere and then the 2-nitroindoles $1(0.1 \mathrm{mmol})$ was added. The mixture was placed for $-20^{\circ} \mathrm{C}$ for $15-20 \mathrm{~min}, \alpha$-phenylisocyano acetates $2(0.12 \mathrm{mmol})$ was dissolved in distilled toluene $(1 \mathrm{~mL})$ was added. After stirring for the specific time at $-20{ }^{\circ} \mathrm{C}$, the reaction mixture was directly purified by flash chromatography on silica gel (petroleum ether/ethyl acetate $=10: 1 \sim 5: 1$ ) to give the desired product 3 .

\section{Reference:}

1. Sladojevich, F.; Trabocchi, A.; Guarna, A.; Dixon, D. A New Family of Cinchona-Derived Amino Phosphine Precatalysts: Application to the Highly Enantio- and Diastereoselective Silver-Catalyzed Isocyanoacetate Aldol Reaction. J. Am. Chem. Soc. 2011, 133, 1710. (b) Shao, P.-L.; Liao, J.-Y.; Ho, Y. A.; Zhao, Y. Highly Diastereoand Enantioselective Silver-Catalyzed Double [3+2] Cyclization of $\alpha$-Imino Esters with Isocyanoacetate. Angew. Chem., Int. Ed. 2014, 53, 5435. 


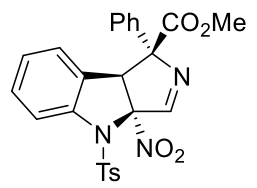

Methyl (1R,3aS,8bS)-3a-nitro-1-phenyl-4-tosyl-1,3a,4,8b-tetrahydropyrrolo[3,4-b]indole-1carboxylate (3a)

The product 3a was purified by flash column chromatography (petroleum ether/ethyl acetate = 7:1). White solid; $48.9 \mathrm{mg}$, 99\% yield; $86: 14 \mathrm{dr}$, 94\% ee; HPLC (IC, $i$-propanol $/ n$-hexane $=30 / 70$, flow rate $=1 \mathrm{~mL} / \mathrm{min}, \lambda=254 \mathrm{~nm}$ ) $t_{\mathrm{R}}=13.180 \mathrm{~min}$ (major), $17.747 \mathrm{~min}$ (minor); $[\alpha]_{\mathrm{D}}{ }^{20}=-88.5(\mathrm{c}$ 1.8, $\mathrm{CH}_{2} \mathrm{Cl}_{2}$ ); m.p. 151.2-152.4 ${ }^{\circ} \mathrm{C} ;{ }^{1} \mathrm{H} \mathrm{NMR}\left(300 \mathrm{MHz}, \mathrm{CDCl}_{3}\right) \delta$ (major diastereoisomer) $\delta 8.64$ (s, 1H), $8.04-7.82(\mathrm{~m}, 2 \mathrm{H}), 7.68(\mathrm{dt}, J=6.4,1.4 \mathrm{~Hz}, 2 \mathrm{H}), 7.57-7.43(\mathrm{~m}, 2 \mathrm{H}), 7.45-7.24(\mathrm{~m}$, $6 \mathrm{H}), 7.15-7.05(\mathrm{~m}, 1 \mathrm{H}), 4.55(\mathrm{~s}, 1 \mathrm{H}), 2.62(\mathrm{~s}, 3 \mathrm{H}), 2.43(\mathrm{~s}, 3 \mathrm{H}){ }^{13} \mathrm{C} \mathrm{NMR}\left(100 \mathrm{MHz}, \mathrm{CDCl}_{3}\right) \delta$ (major diastereoisomer) 167.7, 158.8, 145.7, 140.9, 139.2, 134.8, 130.3, 130.1, 128.7, 128.6, 128.1, 127.1, 126.1, 124.6, 124.0, 114.4, 112.5, 91.5, 64.3, 52.1, 21.7; HRMS (ESI) calcd. for $\mathrm{C}_{25} \mathrm{H}_{21} \mathrm{~N}_{3} \mathrm{NaO}_{6} \mathrm{~S}[\mathrm{M}+\mathrm{Na}]^{+}$514.1043, found: 514.1048 .

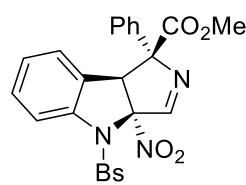

\section{Methyl (1R,3aS,8bS)-3a-Nitro-1-phenyl-4-(phenylsulfonyl)-1,3a,4,8b-tetrahydropyrrolo}

\section{$[3,4 b]$ indole-1-carboxylate (3b)}

The product 3b was purified by flash column chromatography (petroleum ether/ethyl acetate = 7:1). White solid; $43.8 \mathrm{mg}, 92 \%$ yield; $86: 14 \mathrm{dr}$, 93\% ee; HPLC (IC, $i$-propanol $/ n$-hexane $=20 / 80$, flow rate $=1 \mathrm{~mL} / \mathrm{min}, \lambda=254 \mathrm{~nm}$ ) $\mathrm{t}_{\mathrm{R}}=13.480 \mathrm{~min}$ (major), $18.787 \mathrm{~min}$ (minor); $[\alpha]_{\mathrm{D}}{ }^{20}=-84.6(\mathrm{c}$ 1.2, $\mathrm{CH}_{2} \mathrm{Cl}_{2}$ ); m.p. 98.3-99.9 ${ }^{\circ} \mathrm{C} ;{ }^{1} \mathrm{H} \mathrm{NMR}\left(400 \mathrm{MHz}, \mathrm{CDCl}_{3}\right) \delta$ (major diastereoisomer) $8.67(\mathrm{~s}$, 1H), $8.18-7.98(\mathrm{~m}, 2 \mathrm{H}), 7.73-7.68(\mathrm{~m}, 3 \mathrm{H}), 7.66-7.60(\mathrm{~m}, 2 \mathrm{H}), 7.54-7.46(\mathrm{~m}, 2 \mathrm{H}), 7.45-7.32$ $(\mathrm{m}, 4 \mathrm{H}), 7.18-7.11(\mathrm{~m}, 1 \mathrm{H}), 4.58(\mathrm{~s}, 1 \mathrm{H}), 2.62(\mathrm{~s}, 3 \mathrm{H}) ;{ }^{13} \mathrm{C} \mathrm{NMR}\left(100 \mathrm{MHz}, \mathrm{CDCl}_{3}\right) \delta($ major diastereoisomer) 162.9, 153.9, 136.0, 134.4, 133.0, 129.6, 125.6, 124.8, 123.9, 123.8, 123.3, 122.3, 121.4, 119.8, 119.4, 109.6, 107.8, 86.8, 72.6, 59.6, 47.3; HRMS (ESI) calcd. for $\mathrm{C}_{24} \mathrm{H}_{20} \mathrm{~N}_{3} \mathrm{NaO}_{6} \mathrm{~S}$ $[\mathrm{M}+\mathrm{Na}]^{+}$500.0887, found: 500.0905 .

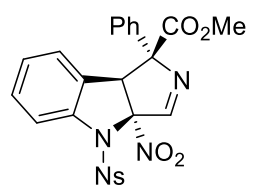

\section{Methyl (1R,3aS,8bS)-3a-Nitro-4-((4-nitrophenyl)sulfonyl)-1-phenyl-1,3a,4,8b- tetrahydropyrrolo[3,4-b]indole-1-carboxylate $(3 \mathrm{c})$}

The product 3c was purified by flash column chromatography (petroleum ether/ethyl acetate $=$ 8:1). Yellow solid; $44.5 \mathrm{mg}, 85 \%$ yield; $88: 12 \mathrm{dr}$, $90 \%$ ee; HPLC (IC, $i$-propanol $/ n$-hexane $=20 / 80$, flow rate $=1 \mathrm{~mL} / \mathrm{min}, \lambda=254 \mathrm{~nm}) \mathrm{t}_{\mathrm{R}}=23.337 \mathrm{~min}$ (major), $39.930 \mathrm{~min}$ (minor); $[\alpha]_{\mathrm{D}}{ }^{20}=-80.9(\mathrm{c}$ 2.1, $\mathrm{CH}_{2} \mathrm{Cl}_{2}$ ); m.p. 84.5-85.4 ${ }^{\circ} \mathrm{C}$; ${ }^{1} \mathrm{H}$ NMR $\left(300 \mathrm{MHz}, \mathrm{CDCl}_{3}\right) \delta$ (major diastereoisomer) $\delta 8.56(\mathrm{~s}$, $1 \mathrm{H}), 8.51-8.35(\mathrm{~m}, 2 \mathrm{H}), 8.33-8.17(\mathrm{~m}, 2 \mathrm{H}), 7.88-7.61(\mathrm{~m}, 2 \mathrm{H}), 7.60-7.30(\mathrm{~m}, 6 \mathrm{H}), 7.17(\mathrm{~m}$, $1 \mathrm{H}), 4.64(\mathrm{~s}, 1 \mathrm{H}), 2.85(\mathrm{~s}, 3 \mathrm{H})$; ${ }^{13} \mathrm{C} \mathrm{NMR}\left(100 \mathrm{MHz}, \mathrm{CDCl}_{3}\right) \delta$ (major diastereoisomer) $\delta 167.7$, 157.9 , 150.9, 143.2, 140.4, 138.8, 130.6, 129.3, 128.8, 128.7, 127.1, 126.4, 124.7, 124.6, 124.6, 
114.2, 112.7, 91.7, 64.1, 52.5; HRMS (ESI) calcd. for $\mathrm{C}_{24} \mathrm{H}_{18} \mathrm{~N}_{4} \mathrm{NaO}_{8} \mathrm{~S}[\mathrm{M}+\mathrm{Na}]^{+} 545.0738$, found: 545.0753 .

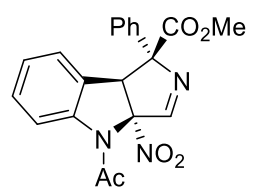

Methyl $(1 R, 3 a R, 8 b S)$-4-acetyl-3a-nitro-1-phenyl-1,3a,4,8b-tetrahydropyrrolo[3,4-b]indole-1carboxylate (3d)

The product 3d was purified by flash column chromatography (petroleum ether/ethyl acetate = 5:1). Yellow solid; $36.4 \mathrm{mg}, 96 \%$ yield; $82: 18 \mathrm{dr}, 92 \%$ ee; HPLC (IC, ethanol $/ n$-hexane $=20 / 80$, flow rate $=1 \mathrm{~mL} / \mathrm{min}, \lambda=254 \mathrm{~nm}$ ) $t_{\mathrm{R}}=12.117 \mathrm{~min}$ (major), $10.257 \mathrm{~min}$ (minor); $[\alpha]_{\mathrm{D}}{ }^{20}=-83.4(\mathrm{c}$ 1.0, $\mathrm{CH}_{2} \mathrm{Cl}_{2}$ ); m.p. $78.8-79.8^{\circ} \mathrm{C} ;{ }^{1} \mathrm{H}$ NMR $\left(300 \mathrm{MHz}, \mathrm{CDCl}_{3}\right) \delta$ (major diastereoisomer) $\delta 8.53(\mathrm{~s}$, 1H), $7.88-7.63(\mathrm{~m}, 2 \mathrm{H}), 7.62-7.28(\mathrm{~m}, 6 \mathrm{H}), 7.28-7.15(\mathrm{~m}, 1 \mathrm{H}), 4.47(\mathrm{~s}, 1 \mathrm{H}), 3.13(\mathrm{~s}, 3 \mathrm{H}), 2.61$ (s, 3H); ${ }^{13} \mathrm{C}$ NMR $\left(75 \mathrm{MHz}, \mathrm{CDCl}_{3}\right.$ ) $\delta$ (major diastereoisomer) 168.7, 168.3, 159.3, 141.1, 139.4, 130.3, 128.6, 128.5, 127.2, 127.0, 126.4, 124.3, 113.6, 113.5, 91.6, 61.5, 52.6, 25.2; HRMS (ESI) calcd. for $\mathrm{C}_{20} \mathrm{H}_{17} \mathrm{~N}_{3} \mathrm{NaO}_{5}[\mathrm{M}+\mathrm{Na}]^{+} 402.1060$, found: 402.1062 .

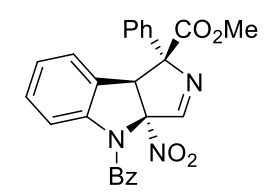

Methyl (1R,3aR,8bS)-4-benzoyl-3a-nitro-1-phenyl-1,3a,4,8b-tetrahydropyrrolo[3,4-b] indole1-carboxylate (3e)

The product 3e was purified by flash column chromatography (petroleum ether/ethyl acetate $=$ 6:1). White solid; $42.7 \mathrm{mg}, 97 \%$ yield; $58: 42 \mathrm{dr}, 80 \%$ ee; HPLC (IC, ethanol $/ n$-hexane $=5 / 95$, flow rate $=1 \mathrm{~mL} / \mathrm{min}, \lambda=254 \mathrm{~nm}$ ) $\mathrm{t}_{\mathrm{R}}=25.227 \min$ (major), $21.940 \min$ (minor); $[\alpha]_{\mathrm{D}^{20}}=-179.4(\mathrm{c} 1.0$, $\mathrm{CH}_{2} \mathrm{Cl}_{2}$ ); m.p. 126.4-127.6 ${ }^{\circ} \mathrm{C}$; ${ }^{1} \mathrm{H}$ NMR $\left(300 \mathrm{MHz}, \mathrm{CDCl}_{3}\right) \delta$ (major diastereoisomer) $\delta 8.57$ (s, $1 \mathrm{H}), 7.98-7.71(\mathrm{~m}, 2 \mathrm{H}), 7.70-7.59(\mathrm{~m}, 3 \mathrm{H}), 7.58-7.36(\mathrm{~m}, 6 \mathrm{H}), 7.19-6.93(\mathrm{~m}, 2 \mathrm{H}), 6.16(\mathrm{~d}, J$ $=7.7 \mathrm{~Hz}, 1 \mathrm{H}), 4.54(\mathrm{~s}, 1 \mathrm{H}), 3.39-2.97(\mathrm{~m}, 3 \mathrm{H}) ;{ }^{13} \mathrm{C} \mathrm{NMR}\left(100 \mathrm{MHz}, \mathrm{CDCl}_{3}\right) \delta$ (major diastereoisomer) 168.5, 168.3 158.9, 141.9, 139.4, 133.7, 132.3, 129.5, 129.1, 128.7, 128.5, 127.9, 127.2, 126.4, 126.1, 124.3, 115.1, 114.0, 91.5, 62.2, 52.8; HRMS (ESI) calcd. for $\mathrm{C}_{25} \mathrm{H}_{19} \mathrm{~N}_{3} \mathrm{NaO}_{5}$ $[\mathrm{M}+\mathrm{Na}]^{+} 464.1217$, found: 464.1223 .

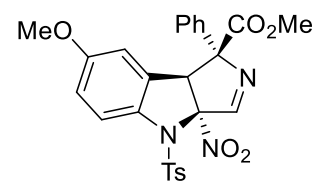

Methyl (1R,3aS,8bS)-7-methoxy-3a-nitro-1-phenyl-4-tosyl-1,3a,4,8b-tetrahydropyrrolo [3,4b]indole-1-carboxylate (3f)

The product $3 \mathbf{f}$ was purified by flash column chromatography (petroleum ether/ethyl acetate $=$ 8:1). White solid; $51.6 \mathrm{mg}, 99 \%$ yield; $76: 24 \mathrm{dr}, 92 \%$ ee; HPLC (IC, ethanol $/ n$-hexane $=1 / 99$, flow rate $=1 \mathrm{~mL} / \mathrm{min}, \lambda=254 \mathrm{~nm}$ ) $\mathrm{t}_{\mathrm{R}}=18.020 \min$ (major), $20.360 \mathrm{~min}\left(\right.$ minor); $[\alpha]_{\mathrm{D}}{ }^{20}=-99.4(\mathrm{c} 1.3$, $\mathrm{CH}_{2} \mathrm{Cl}_{2}$ ); m.p. 139.9-140.7 ${ }^{\circ} \mathrm{C}$; ${ }^{1} \mathrm{H}$ NMR $\left(300 \mathrm{MHz}, \mathrm{CDCl}_{3}\right) \delta$ (major diastereoisomer) $\delta 8.61(\mathrm{~s}$, 1H), $7.96-7.86(\mathrm{~m}, 2 \mathrm{H}), 7.68-7.61(\mathrm{~m}, 2 \mathrm{H}), 7.51-7.43(\mathrm{~m}, 2 \mathrm{H}), 7.43-7.34(\mathrm{~m}, 3 \mathrm{H}), 7.31-7.21$ (m, 2H), $6.93-6.88(\mathrm{~m}, 1 \mathrm{H}), 6.88-6.80(\mathrm{~m}, 1 \mathrm{H}), 4.50(\mathrm{~s}, 1 \mathrm{H}), 3.77(\mathrm{~s}, 3 \mathrm{H}), 2.70(\mathrm{~s}, 3 \mathrm{H}), 2.43(\mathrm{~s}$, 
$3 \mathrm{H}$ ); ${ }^{13} \mathrm{C}$ NMR (75 MHz, $\left.\mathrm{CDCl}_{3}\right) \delta$ (major diastereoisomer) $\delta 167.6,158.7,156.6,145.4,139.2$, 134.9, 134.3, 130.0, 128.7, 128.5, 128.0, 127.0, 125.9, 115.0, 114.7, 113.1, 112.5, 91.2, 64.2, 55.9, 52.1, 21.6; HRMS (ESI) calcd. for $\mathrm{C}_{26} \mathrm{H}_{23} \mathrm{~N}_{3} \mathrm{NaO}_{7} \mathrm{~S}[\mathrm{M}+\mathrm{Na}]^{+} 544.1149$, found: 544.1147.

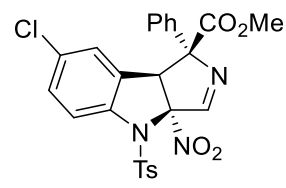

\section{Methyl (1R,3aS,8bS)-7-chloro-3a-nitro-1-phenyl-4-tosyl-1,3a,4,8b-tetrahydropyrrolo[3,4-b] indole-1-carboxylate (3g)}

The product $\mathbf{3 g}$ was purified by flash column chromatography (petroleum ether/ethyl acetate $=$ 7:1). White solid; $52.2 \mathrm{mg}$, 99\% yield; $86: 14 \mathrm{dr}$, 94\% ee; HPLC (IC, $i$-propanol $/ n$-hexane $=3 / 97$, flow rate $=1 \mathrm{~mL} / \mathrm{min}, \lambda=254 \mathrm{~nm}) \mathrm{t}_{\mathrm{R}}=10.733 \mathrm{~min}$ (major), $9.907 \mathrm{~min}$ (minor); $[\alpha]_{\mathrm{D}}{ }^{20}=-88.9(\mathrm{c}$ 1.6, $\mathrm{CH}_{2} \mathrm{Cl}_{2}$ ); m.p. $152.7-153.8{ }^{\circ} \mathrm{C} ;{ }^{1} \mathrm{H}$ NMR $\left(300 \mathrm{MHz}, \mathrm{CDCl}_{3}\right) \delta$ (major diastereoisomer) $\delta 8.60$ (s, 1H), $7.93-7.84(\mathrm{~m}, 2 \mathrm{H}), 7.68-7.60(\mathrm{~m}, 1 \mathrm{H}), 7.56-7.49(\mathrm{~m}, 1 \mathrm{H}), 7.44-7.33(\mathrm{~m}, 5 \mathrm{H}), 7.33-$ $7.28(\mathrm{~m}, 2 \mathrm{H}), 7.09-7.01(\mathrm{~m}, 1 \mathrm{H}), 4.86(\mathrm{~d}, J=1.0 \mathrm{~Hz}, 1 \mathrm{H}), 3.00(\mathrm{~s}, 3 \mathrm{H}), 2.44(\mathrm{~s}, 3 \mathrm{H}) ;{ }^{13} \mathrm{C}$ NMR $\left(75 \mathrm{MHz}, \mathrm{CDCl}_{3}\right) \delta$ (major diastereoisomer) $\delta 167.1,159.1,145.5,141.0,136.2,134.8,133.4,131.6$, 130.3, 130.1, 130.0, 128.0, 128.0, 127.4, 126.7, 124.6, 123.8, 114.7, 112.5, 90.4, 61.0, 52.5, 21.7; HRMS (ESI) calcd. for $\mathrm{C}_{25} \mathrm{H}_{20}{ }^{35} \mathrm{ClN}_{3} \mathrm{NaO}_{6} \mathrm{~S}[\mathrm{M}+\mathrm{Na}]^{+}$548.0654, found: 548.0650; $\mathrm{C}_{25} \mathrm{H}_{20}{ }^{37} \mathrm{ClN}_{3} \mathrm{NaO}_{6} \mathrm{~S}[\mathrm{M}+\mathrm{Na}]^{+}$550.0634; found: 550.0614.

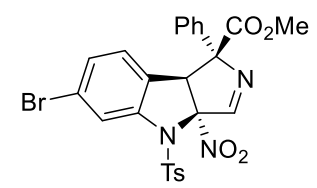

Methyl (1R,3aS,8bS)-6-bromo-3a-nitro-1-phenyl-4-tosyl-1,3a,4,8b-tetrahydropyrrolo[3,4-b] indole-1-carboxylate (3h)

The product $\mathbf{3 h}$ was purified by flash column chromatography (petroleum ether/ethyl acetate = 7:1). White solid; $56.7 \mathrm{mg}$, 99\% yield; 89:11 dr, 94\% ee; HPLC (IC, ethanol $/ n$-hexane $=5 / 95$, flow rate $=1 \mathrm{~mL} / \mathrm{min}, \lambda=254 \mathrm{~nm}$ ) $t_{\mathrm{R}}=15.627 \mathrm{~min}$ (major), $17.850 \mathrm{~min}($ minor $) ;[\alpha]_{\mathrm{D}}{ }^{20}=-150.3(\mathrm{c}$ 1.8, $\mathrm{CH}_{2} \mathrm{Cl}_{2}$ ); m.p.67.7-68.6 ${ }^{\circ} \mathrm{C} ;{ }^{1} \mathrm{H}$ NMR $\left(300 \mathrm{MHz}, \mathrm{CDCl}_{3}\right) \delta$ (major diastereoisomer) $\delta 8.61(\mathrm{~d}, J$ $=0.9 \mathrm{~Hz}, 1 \mathrm{H}), 7.91(\mathrm{~d}, J=8.1 \mathrm{~Hz}, 2 \mathrm{H}), 7.76-7.57(\mathrm{~m}, 2 \mathrm{H}), 7.57-7.35(\mathrm{~m}, 6 \mathrm{H}), 7.32-7.21(\mathrm{~m}$, $2 \mathrm{H}), 4.47(\mathrm{~s}, 1 \mathrm{H}), 2.84-2.63(\mathrm{~m}, 3 \mathrm{H}), 2.45(\mathrm{~s}, 3 \mathrm{H}) ;{ }^{13} \mathrm{C} \mathrm{NMR}\left(75 \mathrm{MHz}, \mathrm{CDCl}_{3}\right) \delta$ (major diastereoisomer) $\delta 167.6,145.4,145.2,138.6,138.4,132.3,129.8,128.3,128.0,128.0,127.6,127.4$, $127.1,126.3,118.8,100.5,84.2,81.2,78.5,57.1,27.5,21.6$; HRMS (ESI) calcd. for $\mathrm{C}_{25} \mathrm{H}_{20}{ }^{79} \mathrm{BrN}_{3} \mathrm{NaO}_{6} \mathrm{~S}[\mathrm{M}+\mathrm{Na}]^{+}$592.0148, found: 592.0156; $\mathrm{C}_{25} \mathrm{H}_{20}{ }^{81} \mathrm{BrN}_{3} \mathrm{NaO}_{6} \mathrm{~S}[\mathrm{M}+\mathrm{Na}]^{+}$ 594.0131, found: 594.0139.

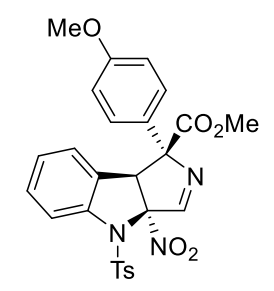

Methyl (1R,3aS,8bS)-1-(4-methoxyphenyl)-3a-nitro-4-tosyl-1,3a,4,8b-tetrahydropyrrolo [3,4b]indole-1-carboxylate (3i) 
The product $3 \mathbf{i}$ was purified by flash column chromatography (petroleum ether/ethyl acetate $=$ 7:1). White solid; $51.8 \mathrm{mg}$, 99\% yield; $87: 13 \mathrm{dr}, 96 \%$ ee; HPLC (IC, ethanol $/ n$-hexane $=20 / 80$, flow rate $=1 \mathrm{~mL} / \mathrm{min}, \lambda=254 \mathrm{~nm}$ ) $t_{\mathrm{R}}=11.810 \mathrm{~min}$ (major), $16.937 \mathrm{~min}$ (minor); $[\alpha]_{\mathrm{D}^{20}}=-106.4(\mathrm{c} 1.9$, $\mathrm{CH}_{2} \mathrm{Cl}_{2}$ ); m.p. $78.9-79.9{ }^{\circ} \mathrm{C} ;{ }^{1} \mathrm{H} \mathrm{NMR}\left(300 \mathrm{MHz}, \mathrm{CDCl}_{3}\right) \delta$ (major diastereoisomer) $\delta 8.63(\mathrm{~s}, 1 \mathrm{H})$, $8.08-7.81(\mathrm{~m}, 2 \mathrm{H}), 7.68-7.51(\mathrm{~m}, 2 \mathrm{H}), 7.45-7.29(\mathrm{~m}, 5 \mathrm{H}), 7.11(\mathrm{ddd}, J=7.5,6.1,2.5 \mathrm{~Hz}, 1 \mathrm{H})$, $7.05-6.93(\mathrm{~m}, 2 \mathrm{H}), 4.65-4.41(\mathrm{~m}, 1 \mathrm{H}), 3.85(\mathrm{~s}, 3 \mathrm{H}), 2.62(\mathrm{~s}, 3 \mathrm{H}), 2.44(\mathrm{~s}, 3 \mathrm{H}){ }^{13} \mathrm{C}$ NMR $(100$ $\mathrm{MHz}, \mathrm{CDCl}_{3}$ ) $\delta$ (major diastereoisomer) 168.0, 159.6, 158.6, 145.6, 140.8, 134.8, 131.3, 130.3, 130.1, 128.3, 128.1, 126.1, 124.6, 124.0, 114.5, 114.0, 112.5, 91.2, 64.5, 55.4, 52.0, 21.7; HRMS (ESI) calcd. for $\mathrm{C}_{26} \mathrm{H}_{23} \mathrm{~N}_{3} \mathrm{NaO}_{7} \mathrm{~S}[\mathrm{M}+\mathrm{Na}]^{+} 544.1149$, found: 544.1148

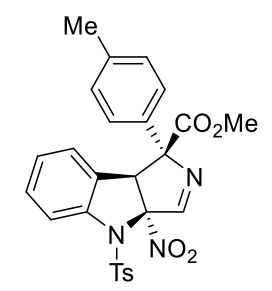

Methyl (1R,3aS,8bS)-3a-nitro-1-(p-tolyl)-4-tosyl-1,3a,4,8b-tetrahydropyrrolo[3,4-b]indole-1carboxylate (3j)

The product $\mathbf{3 j}$ was purified by flash column chromatography (petroleum ether/ethyl acetate $=$ 7:1). White solid; $50.1 \mathrm{mg}$, 99\% yield; $87: 13 \mathrm{dr}, 96 \%$ ee; HPLC (IC, ethanol $/ n$-hexane $=10 / 90$, flow rate $=1 \mathrm{~mL} / \mathrm{min}, \lambda=254 \mathrm{~nm}$ ) $t_{\mathrm{R}}=13.677 \mathrm{~min}$ (major), $19.107 \mathrm{~min}($ minor $) ;[\alpha]_{\mathrm{D}}{ }^{20}=-95.6(\mathrm{c} 1.5$, $\mathrm{CH}_{2} \mathrm{Cl}_{2}$ ); m.p. $72.9-74.0{ }^{\circ} \mathrm{C}$; ${ }^{1} \mathrm{H}$ NMR $\left(300 \mathrm{MHz}, \mathrm{CDCl}_{3}\right) \delta$ (major diastereoisomer) $\delta 8.64(\mathrm{~s}, 1 \mathrm{H})$, $7.99-7.90(\mathrm{~m}, 2 \mathrm{H}), 7.62-7.53(\mathrm{~m}, 2 \mathrm{H}), 7.44-7.25(\mathrm{~m}, 6 \mathrm{H}), 7.17-7.06(\mathrm{~m}, 1 \mathrm{H}), 4.58-4.52(\mathrm{~m}$, $1 \mathrm{H}), 2.63(\mathrm{~s}, 3 \mathrm{H}), 2.45(\mathrm{~s}, 3 \mathrm{H}), 2.41(\mathrm{~s}, 3 \mathrm{H}) ;{ }^{13} \mathrm{C} \mathrm{NMR}\left(100 \mathrm{MHz}, \mathrm{CDCl}_{3}\right) \delta$ (major diastereoisomer) 167.9, 158.7, 145.6, 140.9, 138.4, 136.3, 134.8, 130.2, 130.1, 129.4, 128.1, 126.9, 126.2, 124.7, 124.0, 114.4, 112.5, 91.5, 64.3, 52.0, 21.7, 21.1; HRMS (ESI) calcd. for $\mathrm{C}_{26} \mathrm{H}_{23} \mathrm{~N}_{3} \mathrm{NaO}_{6} \mathrm{~S}[\mathrm{M}+\mathrm{Na}]^{+}$ 528.1200 , found: 528.1196 .

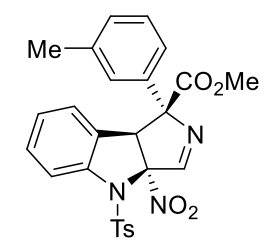

Methyl (1R,3aS,8bS)-3a-nitro-1-(m-tolyl)-4-tosyl-1,3a,4,8b-tetrahydropyrrolo[3,4-b]indole-1carboxylate (3k)

The product 3k was purified by flash column chromatography (petroleum ether/ethyl acetate $=8: 1$ ). White solid; $50.0 \mathrm{mg}, 99 \%$ yield; $87: 13 \mathrm{dr}, 93 \%$ ee; HPLC (IC, ethanol $/ n$-hexane $=10 / 90$, flow rate $=1 \mathrm{~mL} / \mathrm{min}, \lambda=254 \mathrm{~nm}$ ) $t_{\mathrm{R}}=13.120 \mathrm{~min}$ (major), $14.367 \mathrm{~min}$ (minor); $[\alpha]_{\mathrm{D}^{20}}^{20}=-77.8(\mathrm{c}$ 1.6, $\mathrm{CH}_{2} \mathrm{Cl}_{2}$ ); m.p. $61.8-62.9{ }^{\circ} \mathrm{C} ;{ }^{1} \mathrm{H} \mathrm{NMR}\left(300 \mathrm{MHz}, \mathrm{CDCl}_{3}\right) \delta$ (major diastereoisomer) $\delta 8.65(\mathrm{~s}$, $1 \mathrm{H}), 8.02-7.88(\mathrm{~m}, 2 \mathrm{H}), 7.56-7.44(\mathrm{~m}, 2 \mathrm{H}), 7.43-7.26(\mathrm{~m}, 6 \mathrm{H}), 7.26-7.19(\mathrm{~m}, 1 \mathrm{H}), 7.20-7.06$ $(\mathrm{m}, 1 \mathrm{H}), 4.58(\mathrm{~s}, 1 \mathrm{H}), 2.63(\mathrm{~s}, 3 \mathrm{H}), 2.45(\mathrm{~s}, 6 \mathrm{H}) ;{ }^{13} \mathrm{C} \mathrm{NMR}\left(100 \mathrm{MHz}, \mathrm{CDCl}_{3}\right) \delta$ (major diastereoisomer) 167.8, 158.7, 145.6, 140.9, 139.1, 138.4, 134.8, 130.3, 130.1, 129.3, 128.6, 128.1, $127.7,126.2,124.6,124.2,124.0,114.3,112.5,91.6,64.1,52.0,21.7,21.7$; HRMS (ESI) calcd. for $\mathrm{C}_{26} \mathrm{H}_{23} \mathrm{~N}_{3} \mathrm{NaO}_{6} \mathrm{~S}[\mathrm{M}+\mathrm{Na}]^{+}$528.1200, found: 528.1208. 


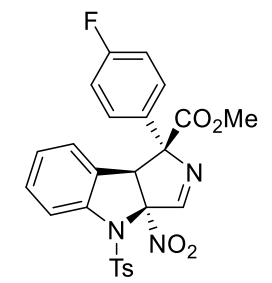

\section{Methyl (1R,3aS,8bS)-1-(4-fluorophenyl)-3a-nitro-4-tosyl-1,3a,4,8b-tetrahydropyrrolo [3,4-} b]indole-1-carboxylate (3I)

The product 31 was purified by flash column chromatography (petroleum ether/ethyl acetate $=$ 8:1). White solid; $47.1 \mathrm{mg}, 98 \%$ yield; $85: 15 \mathrm{dr}$, 92\% ee; HPLC (As, ethanol $/ n$-hexane $=10 / 90$, flow rate $=1 \mathrm{~mL} / \mathrm{min}, \lambda=254 \mathrm{~nm}$ ) $t_{\mathrm{R}}=27.160 \mathrm{~min}$ (major), $20.713 \mathrm{~min}$ (minor); $[\alpha]_{\mathrm{D}}{ }^{20}=+94.8(\mathrm{c}$ 1.5, $\mathrm{CH}_{2} \mathrm{Cl}_{2}$ ); m.p. 91.3-92.5 ${ }^{\circ} \mathrm{C} ;{ }^{1} \mathrm{H}$ NMR $\left(300 \mathrm{MHz}, \mathrm{CDCl}_{3}\right) \delta$ (major diastereoisomer) $\delta 8.64$ (s, $1 \mathrm{H}), 8.04-7.81(\mathrm{~m}, 2 \mathrm{H}), 7.79-7.57(\mathrm{~m}, 2 \mathrm{H}), 7.42-7.36(\mathrm{~m}, 2 \mathrm{H}), 7.36-7.30(\mathrm{~m}, 3 \mathrm{H}), 7.18-7.07$ $(\mathrm{m}, 3 \mathrm{H}), 4.53(\mathrm{~s}, 1 \mathrm{H}), 2.61(\mathrm{~s}, 3 \mathrm{H}), 2.43(\mathrm{~s}, 3 \mathrm{H}){ }^{13} \mathrm{C} \mathrm{NMR}\left(75 \mathrm{MHz}, \mathrm{CDCl}_{3}\right) \delta$ (major diastereoisomer) $\delta 167.5,162.6(\mathrm{~d}, J=246.0 \mathrm{~Hz}), 159.0,145.7,140.8,135.1,135.0(\mathrm{~d}, J=3.0 \mathrm{~Hz})$, 134.7, 130.4, 130.0, 128.9 (d, $J=8.0 \mathrm{~Hz}), 128.1,126.0,124.1(\mathrm{~d}, J=24.8 \mathrm{~Hz}), 124.0,115.6$ (d, $J=$ $21.0 \mathrm{~Hz}), 114.4,112.5,90.9,64.5,52.1,21.6 ;{ }^{19} \mathrm{~F} \mathrm{NMR}\left(376 \mathrm{MHz}, \mathrm{CDCl}_{3}\right) \delta$ (major diastereoisomer) -113.2; HRMS (ESI) calcd. for $\mathrm{C}_{25} \mathrm{H}_{20} \mathrm{FN}_{3} \mathrm{NaO}_{6} \mathrm{~S}[\mathrm{M}+\mathrm{Na}]^{+}$532.0949, found: 532.0946.

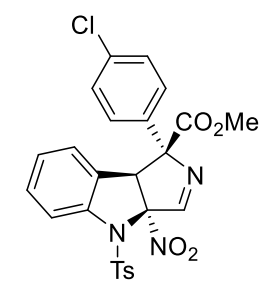

Methyl (1R,3aS,8bS)-1-(4-chlorophenyl)-3a-nitro-4-tosyl-1,3a,4,8b-tetrahydropyrrolo[3,4-b] indole- 1-carboxylate (3m)

The product $3 \mathrm{~m}$ was purified by flash column chromatography (petroleum ether/ethyl acetate $=8: 1$ ). White solid; $51.5 \mathrm{mg}, 99 \%$ yield; $84: 16 \mathrm{dr}, 91 \%$ ee; HPLC (AS, ethanol $/ n$-hexane $=10 / 90$, flow rate $=1 \mathrm{~mL} / \mathrm{min}, \lambda=254 \mathrm{~nm}$ ) $\mathrm{t}_{\mathrm{R}}=9.847 \mathrm{~min}$ (major), $9.119 \mathrm{~min}$ (minor); $[\alpha]_{\mathrm{D}}{ }^{20}=-44.2(\mathrm{c} 1.0$, $\mathrm{CH}_{2} \mathrm{Cl}_{2}$ ); m.p. 71.5-72.6 ${ }^{\circ} \mathrm{C} ;{ }^{1} \mathrm{H}$ NMR $\left(400 \mathrm{MHz}, \mathrm{CDCl}_{3}\right) \delta$ (major diastereoisomer) $8.60(\mathrm{~s}, 1 \mathrm{H})$, $7.93-7.84(\mathrm{~m}, 2 \mathrm{H}), 7.68-7.60(\mathrm{~m}, 1 \mathrm{H}), 7.56-7.49(\mathrm{~m}, 1 \mathrm{H}), 7.44-7.33(\mathrm{~m}, 5 \mathrm{H}), 7.33-7.28(\mathrm{~m}$, 2H), $7.09-7.01(\mathrm{~m}, 1 \mathrm{H}), 4.86(\mathrm{~d}, J=1.0 \mathrm{~Hz}, 1 \mathrm{H}), 3.00(\mathrm{~s}, 3 \mathrm{H}), 2.44(\mathrm{~s}, 3 \mathrm{H}) ;{ }^{13} \mathrm{C} \mathrm{NMR}(100 \mathrm{MHz}$, $\mathrm{CDCl}_{3}$ ) $\delta$ (major diastereoisomer) 167.1, 159.1, 145.5, 141.0, 136.2, 134.8, 133.4, 131.6, 130.3, 130.1, 130.0, 128.0, 128.0, 127.4, 126.7, 124.6, 123.8, 114.7, 112.5, 90.4, 61.0, 52.5, 21.7; HRMS (ESI) calcd. for $\mathrm{C}_{25} \mathrm{H}_{20}{ }^{35} \mathrm{ClN}_{3} \mathrm{NaO}_{6} \mathrm{~S}[\mathrm{M}+\mathrm{Na}]^{+} 548.0654$, found: $548.0663 ; \mathrm{C}_{25} \mathrm{H}_{20}{ }^{37} \mathrm{ClN}_{3} \mathrm{NaO}_{6} \mathrm{~S}$ $[\mathrm{M}+\mathrm{Na}]^{+} 550.0634$, found: 550.0644 .

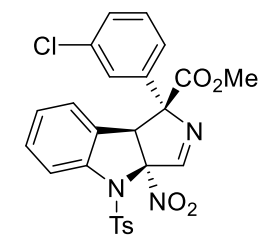

Methyl (1R,3aS,8bS)-1-(3-chlorophenyl)-3a-nitro-4-tosyl-1,3a,4,8b-tetrahydropyrrolo [3,4b]indole-1-carboxylate (3n) 
The product 3n was purified by flash column chromatography (petroleum ether/ethyl acetate $=8: 1$ ). White solid; $52.0 \mathrm{mg}, 99 \%$ yield; $78: 22 \mathrm{dr}, 81 \%$ ee; HPLC (AS, ethanol $/ n$-hexane $=10 / 90$, flow rate $=1 \mathrm{~mL} / \mathrm{min}, \lambda=254 \mathrm{~nm}$ ) $t_{\mathrm{R}}=28.810 \mathrm{~min}$ (major), $21.020 \mathrm{~min}\left(\right.$ minor); $[\alpha]_{\mathrm{D}^{20}}^{20}=-51.2(\mathrm{c}$ 1.5, $\mathrm{CH}_{2} \mathrm{Cl}_{2}$ ); m.p. $168.2-169.4{ }^{\circ} \mathrm{C} ;{ }^{1} \mathrm{H}$ NMR $\left(300 \mathrm{MHz}, \mathrm{CDCl}_{3}\right) \delta$ (major diastereoisomer) $\delta 8.65$ (s, 1H), $8.00-7.85(\mathrm{~m}, 2 \mathrm{H}), 7.70(\mathrm{~d}, J=2.1 \mathrm{~Hz}, 1 \mathrm{H}), 7.55(\mathrm{dt}, J=7.0,1.9 \mathrm{~Hz}, 1 \mathrm{H}), 7.42-7.29(\mathrm{~m}$, 7H), $7.20-7.04(\mathrm{~m}, 1 \mathrm{H}), 4.49$ (s, 1H), 2.61 (s, 3H), 2.44 (s, 3H); ${ }^{13} \mathrm{C}$ NMR $\left(75 \mathrm{MHz}, \mathrm{CDCl}_{3}\right) \delta$ (major diastereoisomer) $\delta 167.2,159.4,145.7,141.1,140.9,134.7,134.7,130.5,130.1,129.9,128.8$, 128.1, 127.5, 126.0, 125.4, 124.2, 124.1, 114.3, 112.6, 91.0, 64.2, 52.2, 21.7; HRMS (ESI) calcd. for $\mathrm{C}_{25} \mathrm{H}_{20}{ }^{35} \mathrm{ClN}_{3} \mathrm{NaO}_{6} \mathrm{~S}[\mathrm{M}+\mathrm{Na}]^{+}$548.0654, found: 548.0664; $\mathrm{C}_{25} \mathrm{H}_{20}{ }^{37} \mathrm{ClN}_{3} \mathrm{NaO}_{6} \mathrm{~S}[\mathrm{M}+\mathrm{Na}]^{+}$ 550.0634, found: 550.0653 .

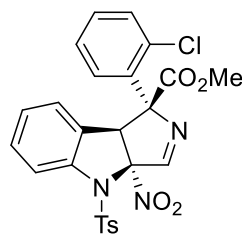

\section{Methyl (1R,3aS,8bS)-1-(2-chlorophenyl)-3a-nitro-4-tosyl-1,3a,4,8b-tetrahydropyrrolo [3,4-b]indole-1-carboxylate (3o)}

The product 30 was purified by flash column chromatography (petroleum ether/ethyl acetate $=$ 7:1). White solid; $51.8 \mathrm{mg}, 82 \%$ yield; $67: 33 \mathrm{dr}, 80 \%$ ee; HPLC (OD, ethanol $/ n$-hexane $=10 / 90$, flow rate $=1 \mathrm{~mL} / \mathrm{min}, \lambda=254 \mathrm{~nm}) \mathrm{t}_{\mathrm{R}}=17.553 \mathrm{~min}$ (major), $26.853 \mathrm{~min}$ (minor); $[\alpha]_{\mathrm{D}^{20}}=-47.1(\mathrm{c}$ 0.7, $\mathrm{CH}_{2} \mathrm{Cl}_{2}$ ); m.p. 81.8-83.0 ${ }^{\circ} \mathrm{C} ;{ }^{1} \mathrm{H}$ NMR $\left(300 \mathrm{MHz}, \mathrm{CDCl}_{3}\right) \delta$ (major diastereoisomer) $\delta 8.60(\mathrm{~s}$, 1H), $7.97-7.80(\mathrm{~m}, 2 \mathrm{H}), 7.72-7.60(\mathrm{~m}, 1 \mathrm{H}), 7.55-7.46(\mathrm{~m}, 1 \mathrm{H}), 7.44-7.32(\mathrm{~m}, 5 \mathrm{H}), 7.34-7.28$ (m, 2H), $7.10-6.98(\mathrm{~m}, 1 \mathrm{H}), 4.85(\mathrm{~s}, 1 \mathrm{H}), 2.99(\mathrm{~s}, 3 \mathrm{H}), 2.43(\mathrm{~s}, 3 \mathrm{H}) ;{ }^{13} \mathrm{C} \mathrm{NMR}\left(75 \mathrm{MHz}, \mathrm{CDCl}_{3}\right)$ $\delta$ (major diastereoisomer) $\delta 167.1,159.1,145.5,141.0,136.2,134.8,133.3,131.5,130.3,130.1$, 130.0, 128.0, 127.9, 127.3, 126.6, 124.6, 123.8, 114.7, 112.5, 90.4, 61.0, 52.5, 21.7; HRMS (ESI) calcd. for $\mathrm{C}_{25} \mathrm{H}_{20} \mathrm{ClN}_{3} \mathrm{NaO}_{6} \mathrm{~S}[\mathrm{M}+\mathrm{Na}]^{+} 548.0654$, found: 548.0660. $\mathrm{C}_{25} \mathrm{H}_{20}{ }^{35} \mathrm{ClN}_{3} \mathrm{NaO}_{6} \mathrm{~S}[\mathrm{M}+\mathrm{Na}]^{+}$ 548.0654, found: 548.0673; $\mathrm{C}_{25} \mathrm{H}_{20}{ }^{37} \mathrm{ClN}_{3} \mathrm{NaO}_{6} \mathrm{~S}[\mathrm{M}+\mathrm{Na}]^{+}$550.0634, found: 550.0656.

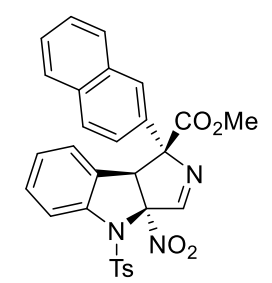

Methyl (1R,3aS,8bS)-1-(naphthalen-2-yl)-3a-nitro-4-tosyl-1,3a,4,8b-tetrahydropyrrolo [3,4b]indole-1-carboxylate (3p)

The product 3p was purified by flash column chromatography (petroleum ether/ethyl acetate = 8:1). White solid; $49.0 \mathrm{mg}, 90 \%$ yield; 83:17 dr, 90\% ee; HPLC (IC, ethanol $/ n$-hexane $=10 / 90$, flow rate $=1 \mathrm{~mL} / \mathrm{min}, \lambda=254 \mathrm{~nm}$ ) $t_{\mathrm{R}}=17.250 \mathrm{~min}$ (major), $19.697 \mathrm{~min}\left(\right.$ minor); $[\alpha]_{\mathrm{D}}{ }^{20}=-260.9(\mathrm{c}$ 0.7, $\mathrm{CH}_{2} \mathrm{Cl}_{2}$ ); m.p. $156.5-157.6{ }^{\circ} \mathrm{C} ;{ }^{1} \mathrm{H}$ NMR $\left(300 \mathrm{MHz}, \mathrm{CDCl}_{3}\right) \delta$ (major diastereoisomer) $\delta 8.71$ $(\mathrm{s}, 1 \mathrm{H}), 8.16(\mathrm{~d}, J=1.9 \mathrm{~Hz}, 1 \mathrm{H}), 8.06-7.83(\mathrm{~m}, 4 \mathrm{H}), 7.77(\mathrm{dd}, J=8.6,2.0 \mathrm{~Hz}, 1 \mathrm{H}), 7.55(\mathrm{dt}, J=$ $6.1,3.3 \mathrm{~Hz}, 2 \mathrm{H}), 7.49-7.43(\mathrm{~m}, 1 \mathrm{H}), 7.43-7.32(\mathrm{~m}, 4 \mathrm{H}), 7.14(\mathrm{ddd}, J=7.6,6.2,2.4 \mathrm{~Hz}, 1 \mathrm{H}), 4.67$ $(\mathrm{d}, J=0.9 \mathrm{~Hz}, 1 \mathrm{H}), 2.64(\mathrm{~s}, 3 \mathrm{H}), 2.44(\mathrm{~s}, 3 \mathrm{H}) ;{ }^{13} \mathrm{C} \mathrm{NMR}\left(75 \mathrm{MHz}, \mathrm{CDCl}_{3}\right) \delta$ (major diastereoisomer) $\delta 167.8,159.0,145.7,140.9,136.4,134.8,133.0,132.9,130.4,130.1,128.6,128.5,128.2,127.6$, 
126.9, 126.7, 126.2, 126.1, 125.0, 124.6, 124.0, 114.4, 112.6, 91.7, 64.1, 52.1, 21.7; HRMS (ESI) calcd. for $\mathrm{C}_{29} \mathrm{H}_{23} \mathrm{~N}_{3} \mathrm{NaO}_{6} \mathrm{~S}[\mathrm{M}+\mathrm{Na}]^{+}$564.1200, found: 564.1201.

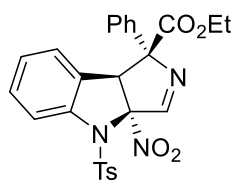

Ethyl (1R,3aS,8bS)-3a-nitro-1-phenyl-4-tosyl-1,3a,4,8b-tetrahydropyrrolo[3,4-b]indole-1carboxylate (3q)

The product $\mathbf{3 q}$ was purified by flash column chromatography (petroleum ether/ethyl acetate $=8: 1$ ). Colorless oil; $47.1 \mathrm{mg}, 93 \%$ yield; $86: 14 \mathrm{dr}, 93 \%$ ee; $\mathrm{HPLC}$ (IC, $i$-propanol $/ n$-hexane $=10 / 90$, flow rate $=1 \mathrm{~mL} / \mathrm{min}, \lambda=254 \mathrm{~nm}$ ) $t_{\mathrm{R}}=10.293 \mathrm{~min}$ (major), $12.598 \mathrm{~min}\left(\right.$ minor); $[\alpha]_{\mathrm{D}}^{20}=-88.0(\mathrm{c}$ 2.3, $\left.\mathrm{CH}_{2} \mathrm{Cl}_{2}\right) ;{ }^{1} \mathrm{H} \mathrm{NMR}\left(300 \mathrm{MHz}, \mathrm{CDCl}_{3}\right) \delta$ (major diastereoisomer) $\delta 8.51(\mathrm{~s}, 0.3 \mathrm{H}), 8.31(\mathrm{~s}, 0.7 \mathrm{H})$, $8.03(\mathrm{~d}, J=8.3 \mathrm{~Hz}, 0.7 \mathrm{H}), 7.77-7.68(\mathrm{~m}, 0.3 \mathrm{H}), 7.63(\mathrm{~d}, J=8.2 \mathrm{~Hz}, 0 \mathrm{H}), 7.56-7.33(\mathrm{~m}, 5 \mathrm{H}), 7.17$ $(\mathrm{t}, J=7.4 \mathrm{~Hz}, 1 \mathrm{H}), 4.61-4.28(\mathrm{~m}, 3 \mathrm{H}), 3.10(\mathrm{~d}, J=5.1 \mathrm{~Hz}, 3 \mathrm{H}), 1.50(\mathrm{t}, J=7.2 \mathrm{~Hz}, 1 \mathrm{H}), 1.44-$ $1.13(\mathrm{~m}, 2 \mathrm{H}){ }^{13} \mathrm{C}$ NMR $\left(100 \mathrm{MHz}, \mathrm{CDCl}_{3}\right) \delta$ (major diastereoisomer) $\delta 167.3,158.6,145.7,141.1$, 139.6, 134.8, 130.3, 130.1, 128.6, 128.5, 128.2, 127.1, 126.3, 124.5, 124.1, 114.5, 112.6, 91.1, 64.3, 62.0, 21.7, 13.0.; HRMS (ESI) calcd. for $\mathrm{C}_{26} \mathrm{H}_{23} \mathrm{~N}_{3} \mathrm{NaO}_{6} \mathrm{~S}$ [M + Na] $]^{+}$528.1200, found: 528.1190.

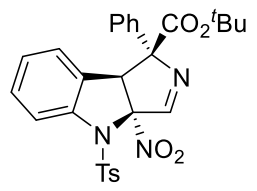

Tert-butyl (1R,3aS,8bS)-3a-Nitro-1-phenyl-4-tosyl-1,3a,4,8b-tetrahydropyrrolo[3,4-b] indole1-carboxylate (3r)

The product $3 \mathbf{r}$ was purified by flash column chromatography (petroleum ether/ethyl acetate $=$ 10:1). White solid; $52.8 \mathrm{mg}, 99 \%$ yield; $81: 19 \mathrm{dr}, 94 \%$ ee; HPLC (IC, $i$-propanol $/ n$-hexane $=1 / 99$, flow rate $=1 \mathrm{~mL} / \mathrm{min}, \lambda=254 \mathrm{~nm}$ ) $t_{\mathrm{R}}=51.170 \mathrm{~min}$ (major), $49.607 \mathrm{~min}$ (minor); $[\alpha]_{\mathrm{D}^{20}}=-64.9(\mathrm{c}$ 0.9, $\mathrm{CH}_{2} \mathrm{Cl}_{2}$ ); m.p. $156.5-157.6{ }^{\circ} \mathrm{C} ;{ }^{1} \mathrm{H}$ NMR $\left(400 \mathrm{MHz}, \mathrm{CDCl}_{3}\right) \delta$ (major diastereoisomer) 8.59 (s, 1H), $7.97-7.91(\mathrm{~m}, 2 \mathrm{H}), 7.70-7.65(\mathrm{~m}, 2 \mathrm{H}), 7.49-7.42(\mathrm{~m}, 3 \mathrm{H}), 7.40-7.32(\mathrm{~m}, 5 \mathrm{H}), 7.16-7.09$ $(\mathrm{m}, 1 \mathrm{H}), 4.52(\mathrm{~d}, J=1.0 \mathrm{~Hz}, 1 \mathrm{H}), 2.43(\mathrm{~s}, 3 \mathrm{H}), 0.82(\mathrm{~s}, 9 \mathrm{H}) ;{ }^{13} \mathrm{C} \mathrm{NMR}\left(100 \mathrm{MHz}, \mathrm{CDCl}_{3}\right) \delta$ (major diastereoisomer) 166.2, 157.9, 145.6, 141.6, 140.2, 134.9, 130.1, 130.1, 128.5, 128.2, 128.1, 127.0, 127.0, 124.6, 124.0, 114.7, 112.7, 90.9, 83.3, 64.2, 26.9, 21.7; HRMS (ESI) calcd. for $\mathrm{C}_{28} \mathrm{H}_{27} \mathrm{~N}_{3} \mathrm{NaO}_{6} \mathrm{~S}[\mathrm{M}+\mathrm{Na}]^{+}$556.1513, found: 556.1514. 
4. Optimization of reaction conditions for the 3-nitroindoles and isocyanoesters

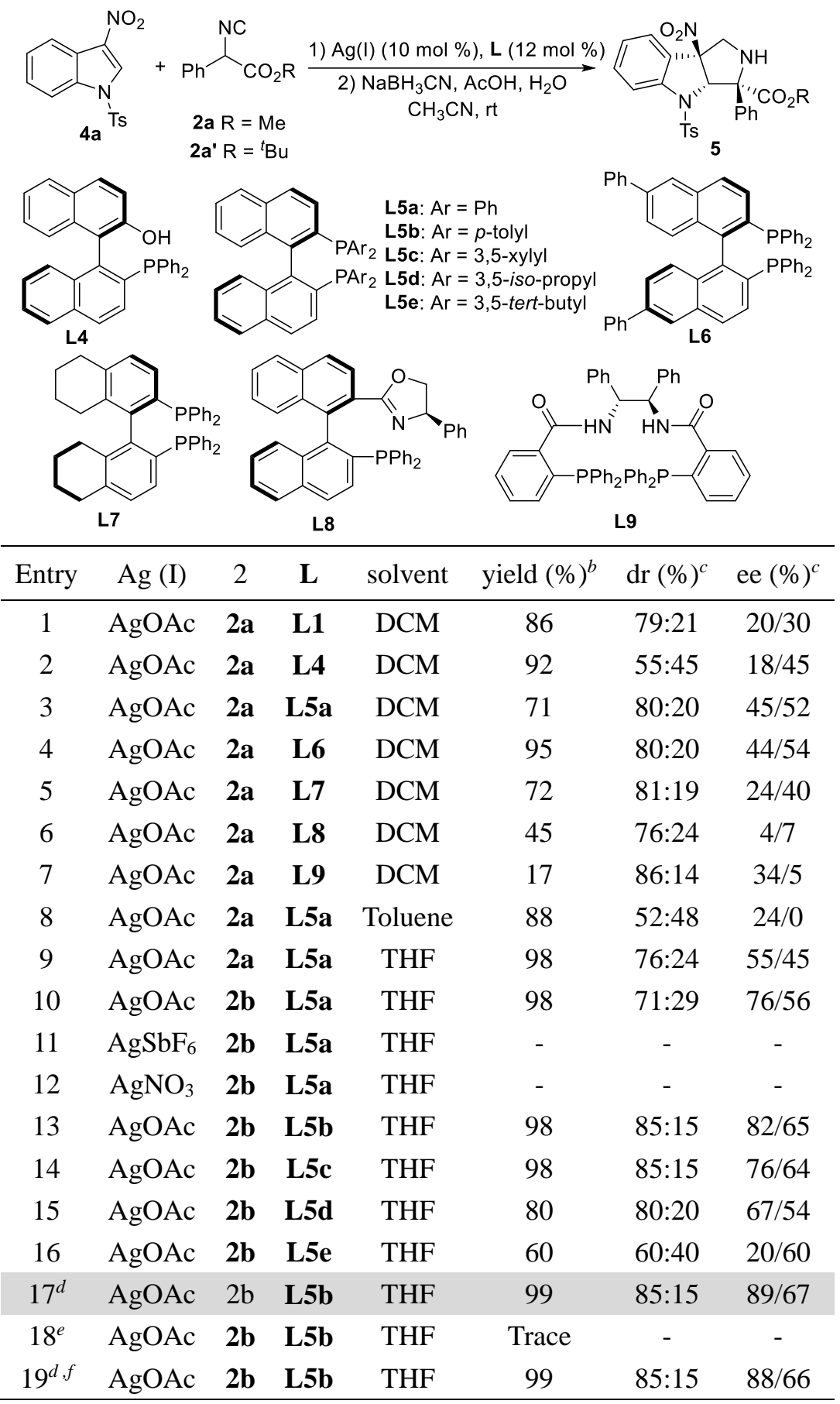

${ }^{a}$ Unless noted, the reactions were carried out with $1 \mathbf{a}(0.1 \mathrm{mmol}), \mathbf{2 a}(0.12 \mathrm{mmol}), 10 \mathrm{~mol} \% \mathrm{Ag}(\mathrm{I})$ catalyst and $12 \mathrm{~mol} \%$ ligand in $2.0 \mathrm{~mL}$ of solvent at room temperature for $12 \mathrm{~h} .{ }^{b}$ Yield of isolated product as a mixture of diastereoisomers. ${ }^{c}$ Determined by chiral HPLC. ${ }^{d}$ Run at $-20{ }^{\circ} \mathrm{C}$. ${ }^{e}$ Run at $30{ }^{\circ} \mathrm{C}^{f}{ }^{f} \mathrm{AgSbF}_{6}(20 \mathrm{~mol} \%)$ and $\mathbf{L 5 b}(24 \mathrm{~mol} \%)$ were used.

\section{General experimental procedures for asymmetric synthesis of compounds 5}

To a flame dried reaction tube were added AgOAc $(1.7 \mathrm{mg}, 10 \mathrm{~mol} \%)$ and chiral ligand $\mathbf{L 5 b}$ $(\boldsymbol{R})$-tol-BINAP $(2.8 \mathrm{mg}, 6 \mathrm{~mol} \%)$, followed by addition freshly distilled THF $(1.0 \mathrm{~mL})$. The reaction solution was stirred at room temperature for $1 \mathrm{~h}$ under an argon atmosphere and then the 3nitroindoles $4(0.1 \mathrm{mmol})$ was added. The mixture was placed for $-20^{\circ} \mathrm{C}$ for $15-20 \mathrm{mins}$, tert-butyl 
2-isocyano-2-phenylacetate 2a' $(0.12 \mathrm{mmol})$ was dissolved in freshly distilled THF (1 mL) was added slowly. After stirring for the specific time at $-20^{\circ} \mathrm{C}$, the reaction mixture was directly purified by flash chromatography on silica gel (petroleum ether/ethyl acetate $=10: 1 \sim 5: 1$ ) to give the crude product. The crude product was dissolved in $\mathrm{CH}_{3} \mathrm{CN}(2 \mathrm{~mL})$ in room temperature with the addition of $\mathrm{H}_{2} \mathrm{O}(50 \mu \mathrm{L})$ and $\mathrm{NaBH}_{3} \mathrm{CN}(12.6 \mathrm{mg}, 2 \mathrm{eq})$, and $\mathrm{HOAc}(50 \mu \mathrm{L})$ in turn. After stirring for $3 \mathrm{~h}$ at room temperature, the mixture was concentrated and purified by flash chromatography on silica gel (petroleum ether/ethyl acetate $=8: 1 \sim 6: 1)$ to give the desired product 5 .

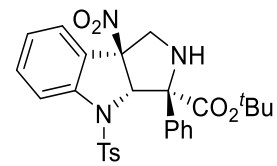

\section{tert-Butyl (3S,3aS,8bS)-8b-nitro-3-phenyl-4-tosyl-1,2,3,3a,4,8b-hexahydropyrrolo[3,4-b] indole-3-carboxylate (5a)}

The product 5a was purified by flash column chromatography (petroleum ether/ethyl acetate $=$ 7:1). White solid; $53.1 \mathrm{mg}, 99 \%$ yield; $85: 15 \mathrm{dr}, 89 \%$ ee; HPLC (OD, $i$-propanol $/ n$-hexane $=5 / 95$, flow rate $=1 \mathrm{~mL} / \mathrm{min}, \lambda=254 \mathrm{~nm}$ ) $\mathrm{t}_{\mathrm{R}}=5.294 \mathrm{~min}$ (major), $4.921 \mathrm{~min}$ (minor); $[\alpha]_{\mathrm{D}}{ }^{20}=-49.5(\mathrm{c} 1.5$, $\mathrm{CH}_{2} \mathrm{Cl}_{2}$ ); m.p. 64.5-65.6 ${ }^{\circ} \mathrm{C} ;{ }^{1} \mathrm{H}$ NMR $\left(300 \mathrm{MHz}, \mathrm{CDCl}_{3}\right) \delta$ (major diastereoisomer) $\delta 8.29-8.03$ (m, 2H), $7.93-7.71(\mathrm{~m}, 1 \mathrm{H}), 7.65-7.22(\mathrm{~m}, 7 \mathrm{H}), 7.08(\mathrm{~d}, J=8.1 \mathrm{~Hz}, 2 \mathrm{H}), 5.93(\mathrm{~s}, 1 \mathrm{H}), 3.69(\mathrm{~d}, J$ $=8.9 \mathrm{~Hz}, 1 \mathrm{H}), 3.33(\mathrm{~d}, J=10.1 \mathrm{~Hz}, 2 \mathrm{H}), 2.31(\mathrm{~s}, 3 \mathrm{H}), 1.51(\mathrm{~s}, 9 \mathrm{H}) ;{ }^{13} \mathrm{C} \mathrm{NMR}\left(75 \mathrm{MHz}, \mathrm{CDCl}_{3}\right) \delta$ (major diastereoisomer) $\delta 170.0,145.3,142.5,139.1,136.6,130.2,130.0,128.7,128.6,127.2,127.0$, 126.0, 124.9, 123.6, 114.3, 113.1, 78.3, 67.5, 54.6, 52.4, 21.7; HRMS (ESI) calcd. for $\mathrm{C}_{28} \mathrm{H}_{29} \mathrm{~N}_{3} \mathrm{O}_{6} \mathrm{~S}$ $[\mathrm{M}+\mathrm{Na}]^{+}$558.1699, found: 558.1674 .

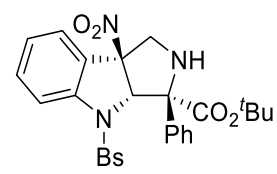

tert-Butyl (3S,3aS,8bS)-4-((4-bromophenyl)sulfonyl)-8b-nitro-3-phenyl-1,2,3,3a,4,8bhexahydropyrrolo[3,4-b]indole-3-carboxylate (5b)

The product $\mathbf{5 b}$ was purified by flash column chromatography (petroleum ether/ethyl acetate = 7:1). White solid; $51.2 \mathrm{mg}, 99 \%$ yield; $82: 18 \mathrm{dr}, 84 \%$ ee; HPLC (OD, $i$-propanol $/ n$-hexane $=5 / 95$, flow rate $=1 \mathrm{~mL} / \mathrm{min}, \lambda=254 \mathrm{~nm}$ ) $t_{\mathrm{R}}=6.497 \mathrm{~min}$ (major), $5.213 \mathrm{~min}$ (minor); $[\alpha]_{\mathrm{D}^{20}}=-53.3(\mathrm{c} 1.4$, $\mathrm{CH}_{2} \mathrm{Cl}_{2}$ ); m.p. 66.5-67.6 ${ }^{\circ} \mathrm{C} ;{ }^{1} \mathrm{H}$ NMR $\left(300 \mathrm{MHz}, \mathrm{CDCl}_{3}\right) \delta$ (major diastereoisomer) $\delta 8.15-7.99$ $(\mathrm{m}, 2 \mathrm{H}), 7.79(\mathrm{~d}, J=8.1 \mathrm{~Hz}, 1 \mathrm{H}), 7.66-7.40(\mathrm{~m}, 7 \mathrm{H}), 7.42-7.18(\mathrm{~m}, 4 \mathrm{H}), 5.90(\mathrm{~s}, 1 \mathrm{H}), 3.66(\mathrm{~d}, J$ $=8.7 \mathrm{~Hz}, 1 \mathrm{H}), 3.31(\mathrm{~d}, J=9.9 \mathrm{~Hz}, 2 \mathrm{H}), 1.50(\mathrm{~s}, 9 \mathrm{H}) ;{ }^{13} \mathrm{C} \mathrm{NMR}\left(75 \mathrm{MHz}, \mathrm{CDCl}_{3}\right) \delta$ (major diastereoisomer) $\delta 167.7,144.1,138.9,135.4,134.0,132.3,129.1,128.3,128.1,128.0,127.9,127.7$, 126.9, 126.6, 118.8, 101.0, 84.1, 80.8, 78.5, 57.3, 27.6; HRMS (ESI) calcd. for $\mathrm{C}_{27} \mathrm{H}_{27} \mathrm{~N}_{3} \mathrm{O}_{6} \mathrm{~S}[\mathrm{M}+$ $\mathrm{Na}]^{+}$544.1513, found: 544.1515 .<smiles>COc1ccc(N(c2ccccc2)c2ccccc2)cc1</smiles>

tert-Butyl (3S,3aS,8bS)-4-((4-methoxyphenyl)sulfonyl)-8b-nitro-3-phenyl-1,2,3,3a,4,8b- 


\section{hexahydropyrrolo[3,4-b]indole-3-carboxylate (5c)}

The product $\mathbf{5} \mathbf{c}$ was purified by flash column chromatography (petroleum ether/ethyl acetate $=$ 7:1). White solid; $54.7 \mathrm{mg}$, 99\% yield; $91: 9 \mathrm{dr}, 81 \%$ ee; HPLC (OD, $i$-propanol $/ n$-hexane $=5 / 95$, flow rate $=1 \mathrm{~mL} / \mathrm{min}, \lambda=254 \mathrm{~nm}$ ) $\mathrm{t}_{\mathrm{R}}=8.813 \mathrm{~min}$ (major), $8.090 \mathrm{~min}$ (minor); $[\alpha]_{\mathrm{D}}{ }^{20}=-47.1(\mathrm{c} 1.6$, $\mathrm{CH}_{2} \mathrm{Cl}_{2}$ ); m.p. $61.8-62.9{ }^{\circ} \mathrm{C} ;{ }^{1} \mathrm{H} \mathrm{NMR}\left(300 \mathrm{MHz}, \mathrm{CDCl}_{3}\right) \delta$ (major diastereoisomer $\delta 8.07(\mathrm{~m}, 2 \mathrm{H})$, $7.84-7.68(\mathrm{~m}, 1 \mathrm{H}), 7.56-7.38(\mathrm{~m}, 6 \mathrm{H}), 7.40-7.24(\mathrm{~m}, 2 \mathrm{H}), 6.87-6.60(\mathrm{~m}, 2 \mathrm{H}), 5.88(\mathrm{~s}, 1 \mathrm{H})$, $3.75(\mathrm{~s}, 3 \mathrm{H}), 3.71-3.59(\mathrm{~m}, 1 \mathrm{H}), 3.39-3.21(\mathrm{~m}, 2 \mathrm{H}), 1.49(\mathrm{~s}, 9 \mathrm{H}) ;{ }^{13} \mathrm{C} \mathrm{NMR}\left(75 \mathrm{MHz}, \mathrm{CDCl}_{3}\right) \delta$ (major diastereoisomer) $\delta 167.7,164.0,144.3,139.0,132.3,129.9,128.3,128.1,128.0,127.9,127.0$, $126.8,126.5,118.8,114.3,101.1,84.0,80.8,78.5,57.3,55.6,27.6$; HRMS (ESI) calcd. for $\mathrm{C}_{28} \mathrm{H}_{28} \mathrm{~N}_{3} \mathrm{O}_{7} \mathrm{~S}[\mathrm{M}+\mathrm{Na}]^{+}$574.1618, found: 574.1620.

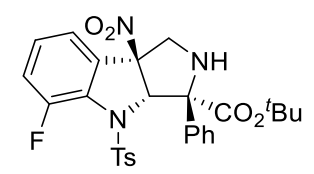

\section{tert-Butyl (3S,3aS,8bS)-5-fluoro-8b-nitro-3-phenyl-4-tosyl-1,2,3,3a,4,8b-hexahydropyrrolo} [3,4-b]indole-3-carboxylate (5d)

The product $\mathbf{5 d}$ was purified by flash column chromatography (petroleum ether/ethyl acetate = 6:1). White solid; $55.0 \mathrm{mg}, 99 \%$ yield; $90: 10 \mathrm{dr}, 91 \%$ ee; HPLC (OD, $i$-propanol $/ n$-hexane $=5 / 95$, flow rate $=1 \mathrm{~mL} / \mathrm{min}, \lambda=254 \mathrm{~nm}$ ) $\mathrm{t}_{\mathrm{R}}=8.577 \min$ (major), $7.313 \min$ (minor); $[\alpha]_{\mathrm{D}^{20}}=-45.5(\mathrm{c} 1.5$, $\mathrm{CH}_{2} \mathrm{Cl}_{2}$ ); m.p. $116.5-117.6{ }^{\circ} \mathrm{C} ;{ }^{1} \mathrm{H}$ NMR $\left(300 \mathrm{MHz}, \mathrm{CDCl}_{3}\right) \delta$ (major diastereoisomer) $\delta 8.05(\mathrm{~m}$, 2H), $7.54-7.36(\mathrm{~m}, 5 \mathrm{H}), 7.36-7.24(\mathrm{~m}, 3 \mathrm{H}), 7.10(\mathrm{~d}, J=7.9 \mathrm{~Hz}, 2 \mathrm{H}), 6.02(\mathrm{~d}, J=4.5 \mathrm{~Hz}, 1 \mathrm{H})$, $3.59(\mathrm{dd}, J=11.5,4.4 \mathrm{~Hz}, 1 \mathrm{H}), 3.44-3.09(\mathrm{~m}, 2 \mathrm{H}), 2.35(\mathrm{~d}, J=4.8 \mathrm{~Hz}, 3 \mathrm{H}), 1.53(\mathrm{~s}, 9 \mathrm{H}) ;{ }^{13} \mathrm{C} \mathrm{NMR}$ $\left(75 \mathrm{MHz}, \mathrm{CDCl}_{3}\right) \delta$ (major diastereoisomer) $\delta 167.6,153.2(\mathrm{~d}, J=257.4 \mathrm{~Hz}), 145.4,138.7,132.2$, $131.7,131.6(\mathrm{~d}, J=9.9 \mathrm{~Hz}), 129.8,128.7(\mathrm{~d}, J=6.75 \mathrm{~Hz}), 128.1(\mathrm{~d}, J=24.8 \mathrm{~Hz}), 128.0,128.0,127.9$, $122.6(\mathrm{~d}, J=3.6 \mathrm{~Hz}), 119.8(\mathrm{~d}, J=19.7 \mathrm{~Hz}), 100.5,84.3,81.9,78.6,58.6,27.5,21.5 ;{ }^{19} \mathrm{~F}$ NMR $(376$ $\mathrm{MHz}, \mathrm{CDCl}_{3}$ ) $\delta$ (major diastereoisomer) -118.1; HRMS (ESI) calcd. for $\mathrm{C}_{28} \mathrm{H}_{28} \mathrm{FN}_{3} \mathrm{O}_{6} \mathrm{~S}[\mathrm{M}+\mathrm{H}]^{+}$ 576.1575, found: 576.1568 .

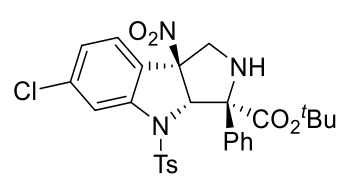

\section{tert-Butyl (3S,3aS,8bS)-6-chloro-8b-nitro-3-phenyl-4-tosyl-1,2,3,3a,4,8b-hexahydropyrrolo [3,4-b]indole-3-carboxylate (5e)}

The product 5e was purified by flash column chromatography (petroleum ether/ethyl acetate $=$ 7:1). White solid; $56.5 \mathrm{mg}$, 99\% yield; $66: 34 \mathrm{dr}$, 90\% ee; HPLC (IC, $i$-propanol $/ n$-hexane $=5 / 95$, flow rate $=1 \mathrm{~mL} / \mathrm{min}, \lambda=254 \mathrm{~nm}$ ) $\mathrm{t}_{\mathrm{R}}=5.140 \mathrm{~min}$ (major), $4.613 \mathrm{~min}$ (minor); $[\alpha]_{\mathrm{D}}{ }^{20}=-167.5(\mathrm{c}$ 1.8, $\mathrm{CH}_{2} \mathrm{Cl}_{2}$ ); m.p. 79.8-80.8 ${ }^{\circ} \mathrm{C} ;{ }^{1} \mathrm{H}$ NMR $\left(300 \mathrm{MHz}, \mathrm{CDCl}_{3}\right) \delta$ (major diastereoisomer) $\delta 8.15-$ $7.95(\mathrm{~m}, 2 \mathrm{H}), 7.93(\mathrm{~d}, J=1.7 \mathrm{~Hz}, 1 \mathrm{H}), 7.52-7.30(\mathrm{~m}, 7 \mathrm{H}), 7.11(\mathrm{~d}, J=8.1 \mathrm{~Hz}, 2 \mathrm{H}), 5.89(\mathrm{~s}, 1 \mathrm{H})$, $3.75-3.54(\mathrm{~m}, 1 \mathrm{H}), 3.31$ (d, $J=4.3 \mathrm{~Hz}, 2 \mathrm{H}), 2.32(\mathrm{~s}, 3 \mathrm{H}), 1.49$ (s, 9H); ${ }^{13} \mathrm{C} \mathrm{NMR}\left(75 \mathrm{MHz}, \mathrm{CDCl}_{3}\right)$ $\delta$ (major diastereoisomer) $\delta 167.5,145.4,145.3,138.6,132.3,130.0,129.8,128.3,128.0,128.0$, 127.6, 126.8, 126.4 121.7, 100.6, 84.2, 81.1, 78.5, 57.1, 27.5, 21.6; HRMS (ESI) calcd. for $\mathrm{C}_{28} \mathrm{H}_{28}{ }^{35} \mathrm{ClN}_{3} \mathrm{O}_{6} \mathrm{~S}[\mathrm{M}+\mathrm{Na}]^{+}$592.1280, found: 592.1284; $\mathrm{C}_{28} \mathrm{H}_{28}{ }^{37} \mathrm{ClN}_{3} \mathrm{O}_{6} \mathrm{~S}[\mathrm{M}+\mathrm{Na}]^{+}$594.1262, found: 594.1264 . 


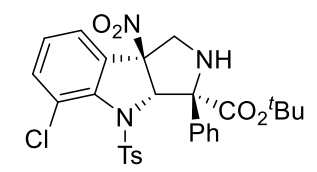

tert-Butyl (3S,3aS,8bS)-5-chloro-8b-nitro-3-phenyl-4-tosyl-1,2,3,3a,4,8b-hexahydropyrrolo [3,4-b]indole-3-carboxylate (5f)

The product $\mathbf{5 f}$ was purified by flash column chromatography (petroleum ether/ethyl acetate $=$ 7:1). White solid; $56.0 \mathrm{mg}$, 98\% yield; $67: 33 \mathrm{dr}$, $85 \%$ ee; HPLC (IC, $i$-propanol $/ n$-hexane $=5 / 95$, flow rate $=1 \mathrm{~mL} / \mathrm{min}, \lambda=254 \mathrm{~nm}$ ) $t_{\mathrm{R}}=8.745 \min$ (major), $5.951 \mathrm{~min}\left(\right.$ minor); $[\alpha]_{\mathrm{D}}{ }^{20}=-87.1(\mathrm{c} 1.1$, $\mathrm{CH}_{2} \mathrm{Cl}_{2}$ ); m.p. $119.9-120.9{ }^{\circ} \mathrm{C}$; ${ }^{1} \mathrm{H}$ NMR $\left(300 \mathrm{MHz}, \mathrm{CDCl}_{3}\right) \delta$ (major diastereoisomer) $\delta 8.19-7.99$ (m, 2H), $7.79(\mathrm{~m}, 1 \mathrm{H}), 7.53-7.33(\mathrm{~m}, 6 \mathrm{H}), 7.32-7.23(\mathrm{~m}, 1 \mathrm{H}), 7.13(\mathrm{~d}, J=8.0 \mathrm{~Hz}, 2 \mathrm{H}), 5.92(\mathrm{~s}$, $1 \mathrm{H}), 3.75-3.54(\mathrm{~m}, 1 \mathrm{H}), 3.33(\mathrm{~d}, J=4.4 \mathrm{~Hz}, 2 \mathrm{H}), 2.33(\mathrm{~s}, 3 \mathrm{H}), 1.51(\mathrm{~s}, 9 \mathrm{H}) ;{ }^{13} \mathrm{C} \mathrm{NMR}(75 \mathrm{MHz}$, $\left.\mathrm{CDCl}_{3}\right) \delta$ (major diastereoisomer) $\delta 167.6,145.4,145.2,138.6,138.4,132.3,129.8,128.3,128.0$, 128.0, 127.6, 127.4, 127.1, 126.3, 118.8, 100.5, 84.2, 81.2, 78.5, 57.1, 27.5, 21.6; HRMS (ESI) calcd. for $\mathrm{C}_{28} \mathrm{H}_{28}{ }^{35} \mathrm{ClN}_{3} \mathrm{O}_{6} \mathrm{~S}[\mathrm{M}+\mathrm{Na}]^{+}$592.1280, found: 592.1284; $\mathrm{C}_{28} \mathrm{H}_{28}{ }^{37} \mathrm{ClN}_{3} \mathrm{O}_{6} \mathrm{~S}[\mathrm{M}+\mathrm{Na}]^{+}$ 594.1262, found: 594.1269 .

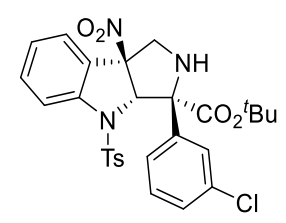

tert-Butyl (3S,3aS,8bS)-3-(3-chlorophenyl)-8b-nitro-4-tosyl-1,2,3,3a,4,8b-hexahydropyrrolo [3,4-b]indole-3-carboxylate $(5 \mathrm{~g})$

The product $\mathbf{5 g}$ was purified by flash column chromatography (petroleum ether/ethyl acetate $=$ 8:1). White solid; $52.6 \mathrm{mg}, 92 \%$ yield; $87: 13 \mathrm{dr}$, $87 \%$ ee; HPLC (IC, $i$-propanol $/ n$-hexane $=5 / 95$, flow rate $=1 \mathrm{~mL} / \mathrm{min}, \lambda=254 \mathrm{~nm}$ ) $t_{\mathrm{R}}=12.439 \mathrm{~min}$ (major), $8.264 \mathrm{~min}$ (minor); $[\alpha]_{\mathrm{D}}{ }^{20}=-49.2(\mathrm{c}$ 1.3, $\mathrm{CH}_{2} \mathrm{Cl}_{2}$ ); m.p. 96.5-97.6 ${ }^{\circ} \mathrm{C} ;{ }^{1} \mathrm{H}$ NMR $\left(300 \mathrm{MHz}, \mathrm{CDCl}_{3}\right) \delta$ (major diastereoisomer) $\delta 8.13-$ $7.97(\mathrm{~m}, 2 \mathrm{H}), 7.76(\mathrm{~d}, J=8.0 \mathrm{~Hz}, 1 \mathrm{H}), 7.55-7.45(\mathrm{~m}, 2 \mathrm{H}), 7.44-7.27(\mathrm{~m}, 5 \mathrm{H}), 7.07(\mathrm{~d}, J=8.0$ $\mathrm{Hz}, 2 \mathrm{H}), 5.81(\mathrm{~s}, 1 \mathrm{H}), 3.68(\mathrm{~d}, J=6.2 \mathrm{~Hz}, 1 \mathrm{H}), 3.31(\mathrm{~d}, J=6.4 \mathrm{~Hz}, 2 \mathrm{H}), 2.30(\mathrm{~s}, 3 \mathrm{H}), 1.50(\mathrm{~s}, 10 \mathrm{H})$; ${ }^{13} \mathrm{C} \mathrm{NMR}\left(75 \mathrm{MHz}, \mathrm{CDCl}_{3}\right) \delta$ (major diastereoisomer) $\delta 167.0,145.2,144.2,141.3,134.2,132.4$, 132.4, 129.7, 129.6, 128.9, 128.2, 127.7, 127.6, 127.3, 126.9, 126.6, 118.7, 101.0, 84.5, 81.0, 78.2, 57.3, 27.6, 21.6; HRMS (ESI) calcd. for $\mathrm{C}_{28} \mathrm{H}_{28}{ }^{35} \mathrm{ClN}_{3} \mathrm{O}_{6} \mathrm{~S}[\mathrm{M}+\mathrm{Na}]^{+}$592.1280, found: 592.1279; $\mathrm{C}_{28} \mathrm{H}_{28}{ }^{37} \mathrm{ClN}_{3} \mathrm{O}_{6} \mathrm{~S}[\mathrm{M}+\mathrm{Na}]^{+}$594.1262, found: 594.1261.

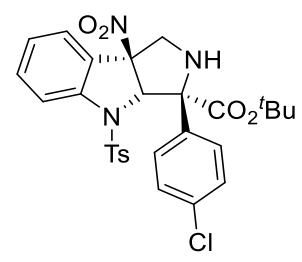

\section{tert-Butyl (3S,3aS,8bS)-3-(4-chlorophenyl)-8b-nitro-4-tosyl-1,2,3,3a,4,8b-hexahydropyrrolo [3,4-b]indole-3-carboxylate (5h)}

The product $\mathbf{5 h}$ was purified by flash column chromatography (petroleum ether/ethyl acetate = 7:1). White solid; $56.7 \mathrm{mg}, 92 \%$ yield; $72: 18 \mathrm{dr}$, $86 \%$ ee; HPLC (IC, $i$-propanol $/ n$-hexane $=5 / 95$, flow rate $=0.5 \mathrm{~mL} / \mathrm{min}, \lambda=254 \mathrm{~nm}$ ) $t_{\mathrm{R}}=9.847 \mathrm{~min}$ (major), $9.119 \mathrm{~min}$ (minor); $[\alpha]_{\mathrm{D}^{20}}=-31.4(\mathrm{c}$ 1.1, $\mathrm{CH}_{2} \mathrm{Cl}_{2}$ ); m.p. 99.5-100.6 ${ }^{\circ} \mathrm{C} ;{ }^{1} \mathrm{H}$ NMR $\left(300 \mathrm{MHz}, \mathrm{CDCl}_{3}\right) \delta$ (major diastereoisomer) $\delta 8.14-$ 
$7.99(\mathrm{~m}, 2 \mathrm{H}), 7.79(\mathrm{~d}, J=8.2 \mathrm{~Hz}, 1 \mathrm{H}), 7.58-7.49(\mathrm{~m}, 2 \mathrm{H}), 7.47-7.41(\mathrm{~m}, 2 \mathrm{H}), 7.41-7.36(\mathrm{~m}$, 2H), $7.33(\mathrm{td}, J=7.6,1.0 \mathrm{~Hz}, 1 \mathrm{H}), 7.09(\mathrm{~d}, J=8.1 \mathrm{~Hz}, 2 \mathrm{H}), 5.82(\mathrm{~s}, 1 \mathrm{H}), 3.76-3.61(\mathrm{~m}, 1 \mathrm{H}), 3.31$ $(\mathrm{t}, J=3.0 \mathrm{~Hz}, 2 \mathrm{H}), 2.33(\mathrm{~s}, 3 \mathrm{H}), 1.52(\mathrm{~s}, 9 \mathrm{H}) ;{ }^{13} \mathrm{C} \mathrm{NMR}\left(75 \mathrm{MHz}, \mathrm{CDCl}_{3}\right) \delta$ (major diastereoisomer) $\delta 167.2,145.2,144.1,137.7,134.0,132.4,129.7,129.7,128.5,127.6,127.6,127.0,126.9,126.6$, 118.7, 101.0, 84.4, 81.0, 78.2, 57.3, 27.6, 21.6; HRMS (ESI) calcd. for $\mathrm{C}_{28} \mathrm{H}_{28}{ }^{35} \mathrm{ClN}_{3} \mathrm{O}_{6} \mathrm{~S}[\mathrm{M}+\mathrm{Na}]^{+}$ 592.1280, found: 592.1283; $\mathrm{C}_{28} \mathrm{H}_{28}{ }^{37} \mathrm{ClN}_{3} \mathrm{O}_{6} \mathrm{~S}[\mathrm{M}+\mathrm{Na}]^{+}$594.1262, found: 594.1276.

\section{Gram scale procedure of compound 3a}

To a flame dried reaction tube were added $\mathrm{AgSbF}_{6}(42.5 \mathrm{mg}, 5 \mathrm{~mol} \%)$ and chiral ligand $\mathbf{L 3}$ (70 $\mathrm{mg}, 6 \mathrm{~mol} \%$ ), followed by addition distilled toluene $(25.0 \mathrm{~mL})$. The reaction solution was stirred at room temperature for $1 \mathrm{~h}$ under an argon atmosphere and then the 2-nitroindole $1 \mathrm{a}(2.5 \mathrm{mmol})$ was added. The mixture was placed for $-20{ }^{\circ} \mathrm{C}$ for $15-20 \mathrm{~min}, \alpha$-phenylisocyano acetate $2 \mathrm{a}$ (3.0 mmol) was dissolved in distilled toluene $(1 \mathrm{~mL})$ was added. After stirring for $24 \mathrm{~h}$ at $-20{ }^{\circ} \mathrm{C}$, the reaction mixture was concentrated and directly purified by flash chromatography on silica gel (petroleum ether/ethyl acetate $=7: 1)$ to give the desired product 3a $(1.22 \mathrm{~g}, 84: 16 \mathrm{dr}, 94 \%$ ee) .

\section{Synthesis of compound 6}

Compound 3a (49.2 mg, $0.1 \mathrm{mmol}$ ) was dissolved in $\mathrm{CH}_{3} \mathrm{CN}$ ( $2 \mathrm{~mL}$ ) in room temperature with the addition of $\mathrm{H}_{2} \mathrm{O}(50 \mu \mathrm{L})$ and $\mathrm{NaBH}_{3} \mathrm{CN}(12.6 \mathrm{mg}, 2 \mathrm{eq})$, and $\mathrm{HOAc}(50 \mu \mathrm{L})$ in sequence. After stirring for $36 \mathrm{~h}$ at room temperature, the mixture was concentrated and purified by flash chromatography on silica gel (petroleum ether/dichloromethane $=3: 1$ ) to give the desired product 6.

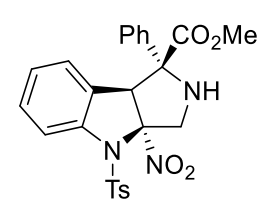

Methyl (1R,3aS,8bS)-3a-nitro-1-phenyl-4-tosyl-1,2,3,3a,4,8b-hexahydropyrrolo[3,4-b]indole1-carboxylate (6)

White solid; $29.5 \mathrm{mg}, 60 \%$ yield; >99:1 dr, $94 \%$ ee; HPLC (IC, $i$-propanol $/ n$-hexane $=30 / 70$, flow rate $=1 \mathrm{~mL} / \mathrm{min}, \lambda=254 \mathrm{~nm}) \mathrm{t}_{\mathrm{R}}=19.054 \mathrm{~min}$ (major), $21.655 \mathrm{~min}$ (minor); $[\alpha]_{\mathrm{D}}{ }^{20}=-80.5(\mathrm{c}$ 1.3, $\mathrm{CH}_{2} \mathrm{Cl}_{2}$ ); m.p. 132.1-133.2 ${ }^{\circ} \mathrm{C} ;{ }^{1} \mathrm{H}$ NMR (300 MHz, $\left.\mathrm{CDCl}_{3}\right) \delta 7.99-7.87(\mathrm{~m}, 2 \mathrm{H}), 7.80-7.63$ $(\mathrm{m}, 2 \mathrm{H}), 7.52(\mathrm{~d}, J=8.3 \mathrm{~Hz}, 1 \mathrm{H}), 7.49-7.34(\mathrm{~m}, 5 \mathrm{H}), 7.33-7.21(\mathrm{~m}, 2 \mathrm{H}), 7.07(\mathrm{~m}, 1 \mathrm{H}), 4.65(\mathrm{~s}$, 1H), 4.32 (dd, $J=14.1,7.2 \mathrm{~Hz}, 1 \mathrm{H}), 4.26-4.08(\mathrm{~m}, 1 \mathrm{H}), 3.25-3.13(\mathrm{~m}, 4 \mathrm{H}), 2.45(\mathrm{~s}, 3 \mathrm{H}) ;{ }^{13} \mathrm{C}$ $\operatorname{NMR}\left(75 \mathrm{MHz}, \mathrm{CDCl}_{3}\right) \delta 170.0,145.3,142.5,139.1,136.6,130.2,130.0,128.7,127.2,127.0,126.0$, 124.9, 123.6, 114.3, 113.1, 78.3, 67.4, 54.6, 52.4, 21.7; HRMS (ESI) calcd. for $\mathrm{C}_{25} \mathrm{H}_{24} \mathrm{~N}_{3} \mathrm{O}_{6} \mathrm{~S}[\mathrm{M}+$ $\mathrm{H}]^{+}$494.1380, found: 494.1378 .

\section{Synthesis of compound 7}

Compound 3a (49.2 mg, $0.1 \mathrm{mmol})$ was dissolved in chloroform $(1 \mathrm{~mL})$ in room temperature with addition of NBS $(21.3 \mathrm{mg}, 1.2 \mathrm{eq})$ and $\mathrm{TsOH} \cdot \mathrm{H}_{2} \mathrm{O}(4.0 \mathrm{mg}, 0.2 \mathrm{eq})$ in sequence. After stirring for $36 \mathrm{~h}$ at room temperature, the mixture was concentrated and purified by flash chromatography on silica gel (petroleum ether/ethyl acetate $=10: 1$ ) to give the desired product 7 .

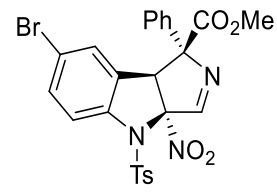


White solid; $22.6 \mathrm{mg}, 40 \%$ yield; $>99: 1 \mathrm{dr}$, $93 \%$ ee; HPLC (IC, $i$-propanol $/ n$-hexane $=30 / 70$, flow rate $=1 \mathrm{~mL} / \mathrm{min}, \lambda=254 \mathrm{~nm}$ ) $t_{\mathrm{R}}=12.418 \mathrm{~min}$ (major), $11.381 \mathrm{~min}$ (minor); $[\alpha]_{\mathrm{D}}{ }^{20}=-98.3(\mathrm{c}$ 0.8, $\left.\mathrm{CH}_{2} \mathrm{Cl}_{2}\right)$; m.p. $156.4-157.5^{\circ} \mathrm{C} ;{ }^{1} \mathrm{H}$ NMR $\left(300 \mathrm{MHz}, \mathrm{CDCl}_{3}\right) \delta 8.61(\mathrm{~s}, 1 \mathrm{H}), 7.95-7.84(\mathrm{~m}, 2 \mathrm{H})$, $7.74-7.55(\mathrm{~m}, 2 \mathrm{H}), 7.52-7.35(\mathrm{~m}, 7 \mathrm{H}), 7.24-7.19(\mathrm{~m}, 1 \mathrm{H}), 4.51(\mathrm{~s}, 1 \mathrm{H}), 2.80(\mathrm{~s}, 3 \mathrm{H}), 2.44(\mathrm{~s}$, $3 \mathrm{H}) ;{ }^{13} \mathrm{C}$ NMR $\left(75 \mathrm{MHz}, \mathrm{CDCl}_{3}\right) \delta 167.5,158.4,145.9,140.1,138.8,134.4,133.2,130.1,129.1$, 128.8, 128.7, 128.0, 126.9, 126.7, 116.5, 114.3, 113.9, 91.4, 63.6, 52.3, 21.7; HRMS (ESI) calcd. for $\mathrm{C}_{25} \mathrm{H}_{20}{ }^{79} \mathrm{BrN}_{3} \mathrm{NaO}_{6} \mathrm{~S}[\mathrm{M}+\mathrm{Na}]^{+} 592.0148$, found: 592.0129; $\mathrm{C}_{25} \mathrm{H}_{20}{ }^{81} \mathrm{BrN}_{3} \mathrm{NaO}_{6} \mathrm{~S}[\mathrm{M}+\mathrm{Na}]^{+}$ 594.0131, found: 594.0114.

\section{Synthesis of compound 8}

Compound 3a (49.2 mg, $0.1 \mathrm{mmol})$ was dissolved in dichloromethane $(1 \mathrm{~mL})$ with addition of DBU (72.8 mg, 5 eq). After stirring for $36 \mathrm{~h}$ at room temperature, the resulting mixture was then quenched with $10 \mathrm{~mL}$ saturated $\mathrm{NH}_{4} \mathrm{Cl}$ solution and extracted with DCM three times. The combined organic layers were dried over $\mathrm{Na}_{2} \mathrm{SO}_{4}$, filtered and concentrated. The crude was purified by flash chromatography on silica gel (petroleum ether/ethyl acetate $=5: 1$ ) to give the desired product 8 (37.9 $\mathrm{mg}, 85 \%$ yield).

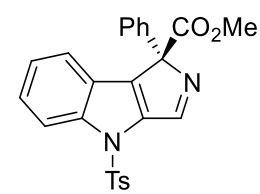

Methyl ( $S$ )-1-phenyl-4-tosyl-1,4-dihydropyrrolo[3,4-b]indole-1-carboxylate (8)

White solid; 99\% ee; HPLC (IC, $i$-propanol $/ n$-hexane $=30 / 70$, flow rate $=1 \mathrm{~mL} / \mathrm{min}, \lambda=254$ $\mathrm{nm}) \mathrm{t}_{\mathrm{R}}=12.447$ min (major), 14.516 min (minor); $[\alpha]_{\mathrm{D}}{ }^{20}=+173.9$ (c 1.3, $\mathrm{CH}_{2} \mathrm{Cl}_{2}$ ); m.p. 156.5$157.6{ }^{\circ} \mathrm{C} ;{ }^{1} \mathrm{H}$ NMR $\left(300 \mathrm{MHz}, \mathrm{CDCl}_{3}\right) \delta 8.84(\mathrm{~d}, J=1.1 \mathrm{~Hz}, 1 \mathrm{H}), 8.13-8.01(\mathrm{~m}, 1 \mathrm{H}), 7.88-7.81$ (m, 1H), $7.81-7.70(\mathrm{~m}, 2 \mathrm{H}), 7.59-7.50(\mathrm{~m}, 2 \mathrm{H}), 7.50-7.36(\mathrm{~m}, 2 \mathrm{H}), 7.37-7.28(\mathrm{~m}, 3 \mathrm{H}), 7.29$ $-7.20(\mathrm{~m}, 2 \mathrm{H}), 3.73(\mathrm{~d}, J=1.2 \mathrm{~Hz}, 3 \mathrm{H}), 2.35(\mathrm{~s}, 3 \mathrm{H}) ;{ }^{13} \mathrm{C} \mathrm{NMR}\left(75 \mathrm{MHz}, \mathrm{CDCl}_{3}\right) \delta 168.9,154.4$, 145.7, 142.9, 142.1, 140.9, 135.7, 134.4, 130.2, 128.7, 128.6, 127.5, 127.0, 126.5, 125.1, 124.7, 121.9, 115.1, 84.4, 53.3, 21.6; HRMS (ESI) calcd. for $\mathrm{C}_{25} \mathrm{H}_{20} \mathrm{~N}_{2} \mathrm{NaO}_{4} \mathrm{~S}[\mathrm{M}+\mathrm{Na}]^{+} 467.1036$, found: 467.1046.

\section{Synthesis of compound 9}

To compound 8 ( $44.4 \mathrm{mg}, 0.1 \mathrm{mmol})$ in $2 \mathrm{~mL}$ of ethanol was added $\mathrm{NaBH}_{4}(37.8 \mathrm{mg}, 1.0 \mathrm{mmol})$ and stirred overnight at room temperature. The resulting mixture was then quenched with $10 \mathrm{~mL}$ saturated $\mathrm{NH}_{4} \mathrm{Cl}$ solution and extracted with DCM three times. The combined organic layers were dried over $\mathrm{Na}_{2} \mathrm{SO}_{4}$, filtered and concentrated. The crude product was dissolved in DCM (1 mL) and cooled to $0{ }^{\circ} \mathrm{C}$ with the addition of piperidine ( $16 \mu \mathrm{L}, 0.2 \mathrm{~mol}, 2.0$ equiv), $\mathrm{Et}_{3} \mathrm{~N}(28 \mu \mathrm{L}, 0.2 \mathrm{mmol}$, 2.0 equiv) and $\mathrm{Boc}_{2} \mathrm{O}$ (46 $\mu \mathrm{L}, 0.2 \mathrm{mmol}, 2.0$ equiv), DMAP (2.4 mg, $20 \mathrm{~mol} \%$,) in turn. Then the mixture was warmed to room temperature. After stirring for overnight, the reaction mixture was directly purified by flash chromatography on silica gel (petroleum ether/ethyl acetate $=15: 1$ ) to give the desired product 9 .

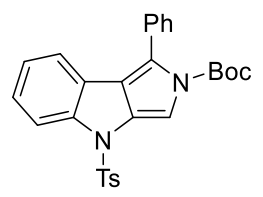


tert-Butyl-1-phenyl-4-tosylpyrrolo[3,4-b]indole-2(4H)-carboxylate (9)

White solid; $43.6 \mathrm{mg}$, $90 \%$ yield; m.p. 156.5-157.6 ${ }^{\circ} \mathrm{C} ;{ }^{1} \mathrm{H}$ NMR $\left(300 \mathrm{MHz}, \mathrm{CDCl}_{3}\right) \delta 7.97(\mathrm{~d}$, $J=8.3 \mathrm{~Hz}, 1 \mathrm{H}), 7.78(\mathrm{~d}, J=8.3 \mathrm{~Hz}, 2 \mathrm{H}), 7.56(\mathrm{~s}, 1 \mathrm{H}), 7.49-7.30(\mathrm{~m}, 5 \mathrm{H}), 7.29-7.23(\mathrm{~m}, 2 \mathrm{H})$, $7.18(\mathrm{~d}, J=8.0 \mathrm{~Hz}, 2 \mathrm{H}), 7.08-7.00(\mathrm{~m}, 1 \mathrm{H}), 2.32(\mathrm{~s}, 3 \mathrm{H}), 1.41(\mathrm{~s}, 9 \mathrm{H}) ;{ }^{13} \mathrm{C} \mathrm{NMR}\left(75 \mathrm{MHz}, \mathrm{CDCl}_{3}\right)$ $\delta$ 149.7, 144.8, 143.7, 134.3, 133.0, 131.4, 129.8, 129.7, 129.4, 127.9, 127.7, 126.9, 126.8, 123.8, 123.7, 123.1, 120.8, 114.7, 103.0, 84.3, 27.6, 21.6; HRMS (ESI) calcd. for $\mathrm{C}_{28} \mathrm{H}_{26} \mathrm{~N}_{2} \mathrm{NaO}_{4} \mathrm{~S}[\mathrm{M}+$ $\mathrm{Na}]^{+}$509.1505, found: 509.1513 .

\section{Synthesis of compound 10}

Compound 8 (44.4 mg, $0.1 \mathrm{mmol}$ ) was dissolved in $\mathrm{CH}_{3} \mathrm{CN}(2 \mathrm{~mL})$ in room temperature with the addition of $\mathrm{H}_{2} \mathrm{O}(50 \mu \mathrm{L})$ and $\mathrm{NaBH}_{3} \mathrm{CN}(12.6 \mathrm{mg}, 2 \mathrm{eq})$, and $\mathrm{HOAc}(50 \mu \mathrm{L})$ in turn. After stirring for $36 \mathrm{~h}$ at room temperature, the mixture was concentrated and purified by flash chromatography on silica gel (petroleum ether/ethyl acetate $=10: 1$ ) to give the desired product $\mathbf{1 0}$.

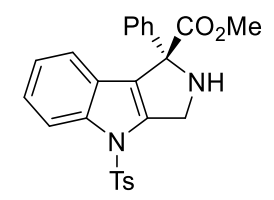

Methyl (S)-1-phenyl-4-tosyl-1,2,3,4-tetrahydropyrrolo[3,4-b]indole-1-carboxylate (10)

White solid; $44.4 \mathrm{mg}$, 99\% yield; 99\% ee; HPLC (IC, $i$-propanol $/ n$-hexane $=1 / 99$, flow rate $=$ $1 \mathrm{~mL} / \mathrm{min}, \lambda=254 \mathrm{~nm}$ ) $\mathrm{t}_{\mathrm{R}}=8.016 \mathrm{~min}$ (major), $9.250 \mathrm{~min}$ (minor); $[\alpha]_{\mathrm{D}}{ }^{20}=-144.2$ (c 1.1, $\mathrm{CH}_{2} \mathrm{Cl}_{2}$ ); m.p. 156.5-157.6 ${ }^{\circ} \mathrm{C} ;{ }^{1} \mathrm{H}$ NMR $\left(300 \mathrm{MHz}, \mathrm{CDCl}_{3}\right) \delta 8.11-7.94(\mathrm{~m}, 1 \mathrm{H}), 7.87-7.72(\mathrm{~m}, 2 \mathrm{H}), 7.65$ $-7.48(\mathrm{~m}, 1 \mathrm{H}), 7.41-7.13(\mathrm{~m}, 10 \mathrm{H}), 4.64(\mathrm{~d}, J=15.3 \mathrm{~Hz}, 1 \mathrm{H}), 4.52(\mathrm{~d}, J=15.4 \mathrm{~Hz}, 1 \mathrm{H}), 3.73(\mathrm{~s}$, $3 \mathrm{H}), 2.38(\mathrm{~s}, 3 \mathrm{H}) ;{ }^{13} \mathrm{C} \mathrm{NMR}\left(75 \mathrm{MHz}, \mathrm{CDCl}_{3}\right) \delta 173.8,145.3,143.8,142.0,140.3,135.1,130.1$, 128.8, 128.0, 126.8, 126.1, 125.6, 124.0, 123.8, 123.7, 120.6, 114.2, 73.3, 52.8, 47.7, 21.6; HRMS (ESI) calcd. for $\mathrm{C}_{25} \mathrm{H}_{20} \mathrm{~N}_{2} \mathrm{NaO}_{4} \mathrm{~S}[\mathrm{M}+\mathrm{H}]^{+} 447.1373$, found: 447.1367 .

\section{X-ray Single Crystal Data for 3a and rac-5d}

Single crystals of compound 3a and 5d were prepared from the mixture solvent of dichloromethane and $\mathrm{EtOH}(v: v=1: 15)$ at room temperature. A suitable crystal was selected for structure determination on a Xcalibur, Eos, Gemini diffractometer. The crystal was kept at 293(2) $\mathrm{K}$ during data collection. Using Olex $2^{[1]}$, the structure was solved with the ShelXS ${ }^{[2]}$ structure solution program using Direct Methods and refined with the ShelXL ${ }^{[3]}$ refinement package using Least Squares minimisation.

[1] Dolomanov, O. V.; Bourhis, L. J.; Gildea, R. J, Howard, J. A. K.; Puschmann, H. 2009, J. Appl. Cryst. 42, 339-341.

[2] Sheldrick, G. M. 2008, Acta Cryst. A64, 112-122.

[3] Sheldrick, G. M. 2015, Acta Cryst. C71, 3-8.

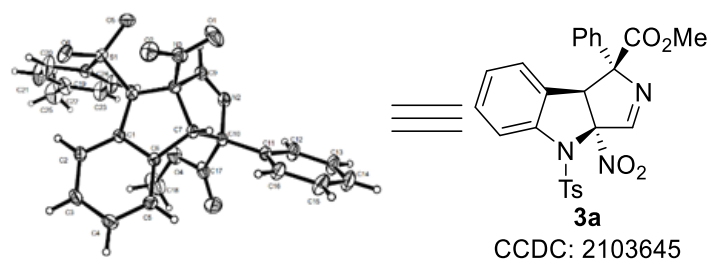

ORTEP of 3a (at $50 \%$ level)

Crystal data and structure refinement for $3 a$. 
Empirical formula

Formula weight

Temperature/K

Crystal system

Space group

$\mathrm{a} / \AA$

$\mathrm{b} / \AA$

$\mathrm{c} / \AA$

$\alpha /{ }^{\circ}$

$\beta /{ }^{\circ}$

$\gamma /{ }^{\circ}$

Volume $/ \AA^{3}$

Z

$$
\begin{gathered}
\rho_{\text {calc }} \mathrm{g} / \mathrm{cm}^{3} \\
\mu / \mathrm{mm}^{-1}
\end{gathered}
$$

$\mathrm{F}(000)$

Crystal size $/ \mathrm{mm}^{3}$

Radiation

$2 \Theta$ range for data collection/ ${ }^{\circ}$

Index ranges

Reflections collected

Independent reflections

Data/restraints/parameters

Goodness-of-fit on $\mathrm{F}^{2}$

Final $R$ indexes $[\mathrm{I}>=2 \sigma(\mathrm{I})]$

Final $\mathrm{R}$ indexes [all data]

Largest diff. peak/hole / e $\AA^{-3}$
$\mathrm{C}_{25} \mathrm{H}_{21} \mathrm{~N}_{3} \mathrm{O}_{6} \mathrm{~S}$

491.51

291(2)

orthorhombic

$\mathrm{P} 2{ }_{1} 2{ }_{1}{ }_{1}$

$11.7947(2)$

13.7557(2)

14.5939(2)

90

90

90

2367.78(6)

4

1.379

1.617

1024.0

$0.250 \times 0.240 \times 0.220$

$\mathrm{CuK} \alpha(\lambda=1.54184)$

8.834 to 142.662

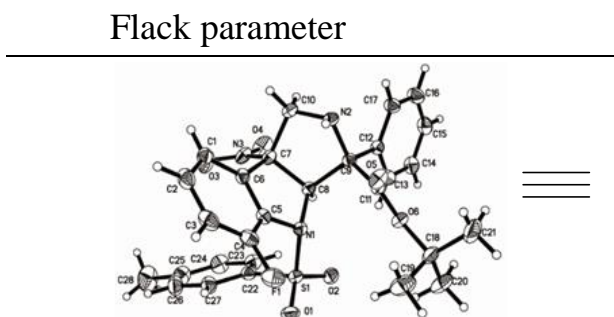

8347

$4418\left[R_{\text {int }}=0.0302, R_{\text {sigma }}=0.0372\right]$

$4418 / 0 / 318$

1.058

$\mathrm{R}_{1}=0.0418, \mathrm{wR}_{2}=0.1168$

$\mathrm{R}_{1}=0.0438, \mathrm{wR}_{2}=0.1192$

$0.53 /-0.31$

$0.009(14)$

ORTEP of 5d (at 50\% level)

Crystal data and structure refinement for $5 \mathrm{~d}$.

Identification code

Empirical formula

Formula weight

Temperature/K

Crystal system
$5 d$

$\mathrm{C}_{28} \mathrm{H}_{28} \mathrm{FN}_{3} \mathrm{O}_{6} \mathrm{~S}$

553.59

293(2)

triclinic 


\begin{tabular}{|c|c|}
\hline Space group & $\mathrm{P}-1$ \\
\hline $\mathrm{a} / \AA ̊$ & $10.9058(4)$ \\
\hline $\mathrm{b} / \AA ̊ \AA$ & $11.6137(5)$ \\
\hline $\mathrm{c} / \AA$ & $11.8341(5)$ \\
\hline$\alpha /^{\circ}$ & $75.357(4)$ \\
\hline$\beta /{ }^{\circ}$ & $73.082(3)$ \\
\hline$\gamma /{ }^{\circ}$ & $82.823(3)$ \\
\hline Volume $/ \AA^{3}$ & $1385.29(10)$ \\
\hline $\mathrm{Z}$ & 2 \\
\hline$\rho_{\text {calc } g / \mathrm{cm}^{3}}$ & 1.327 \\
\hline$\mu / \mathrm{mm}^{-1}$ & 1.494 \\
\hline $\mathrm{F}(000)$ & 580.0 \\
\hline Crystal size $/ \mathrm{mm}^{3}$ & $0.15 \times 0.13 \times 0.1$ \\
\hline Radiation & $\mathrm{CuK} \alpha(\lambda=1.54184)$ \\
\hline ge for data collection ${ }^{\circ}$ & 7.88 to 134.146 \\
\hline Index ranges & $-13 \leq \mathrm{h} \leq 13,-13 \leq \mathrm{k} \leq 13,-14 \leq 1 \leq 14$ \\
\hline flections collected & 19126 \\
\hline ependent reflections & $4924\left[R_{\text {int }}=0.0407, R_{\text {sigma }}=0.0296\right]$ \\
\hline restraints/parameters & $4924 / 0 / 360$ \\
\hline odness-of-fit on $\mathrm{F}^{2}$ & 1.048 \\
\hline $\mathrm{R}$ indexes $[\mathrm{I}>=2 \sigma(\mathrm{I})]$ & $\mathrm{R}_{1}=0.0449, \mathrm{wR}_{2}=0.1241$ \\
\hline $1 \mathrm{R}$ indexes [all data] & $\mathrm{R}_{1}=0.0527, \mathrm{wR}_{2}=0.1319$ \\
\hline t diff. peak/hole / e $\AA^{-3}$ & $0.27 /-0.24$ \\
\hline
\end{tabular}

\section{Determination of the absolute configuration of 5d by ECD spectrum}

\subsection{Computational details}

Based on the experimental crystal structure of enantiomers, there are only two possible configurations $(\boldsymbol{C} 7 \boldsymbol{S}, \boldsymbol{C} 8 \boldsymbol{R}, \boldsymbol{C 1 0 R})-\mathbf{5 d}$ and $(\boldsymbol{C} 7 \boldsymbol{R}, \boldsymbol{C} 8 \mathrm{~S}, \boldsymbol{C} 10 S)-5 \mathrm{~d}$. To further determine the precise configuration of our chiral product, theoretical calculation of the ECD spectrum has been performed and compared with the experimental spectrum. For the ECD spectrum calculations, all structures were optimized at CAM-B3LYP ${ }^{1}$ level of theory with Def2SVP ${ }^{2}$ basis set for all atoms. Empirical dispersion correction has been considered by using Grimme's DFT empirical dispersion correction with the Becke-Jonson (D3BJ) damping function. ${ }^{3}$ Optimized minima were verified at the same level of theory by harmonic vibrational analysis to have no imaginary frequency. Solvent effect was included by using polarizable continuum model ${ }^{4}$ (PCM) with solvent parameters of methanol. CD spectra were calculated by TDDFT at the CAM-B3LYP/Def2SVP level. All DFT geometry optimizations and TDDFT calculations were performed with Gaussian 09 program. ${ }^{5}$ 


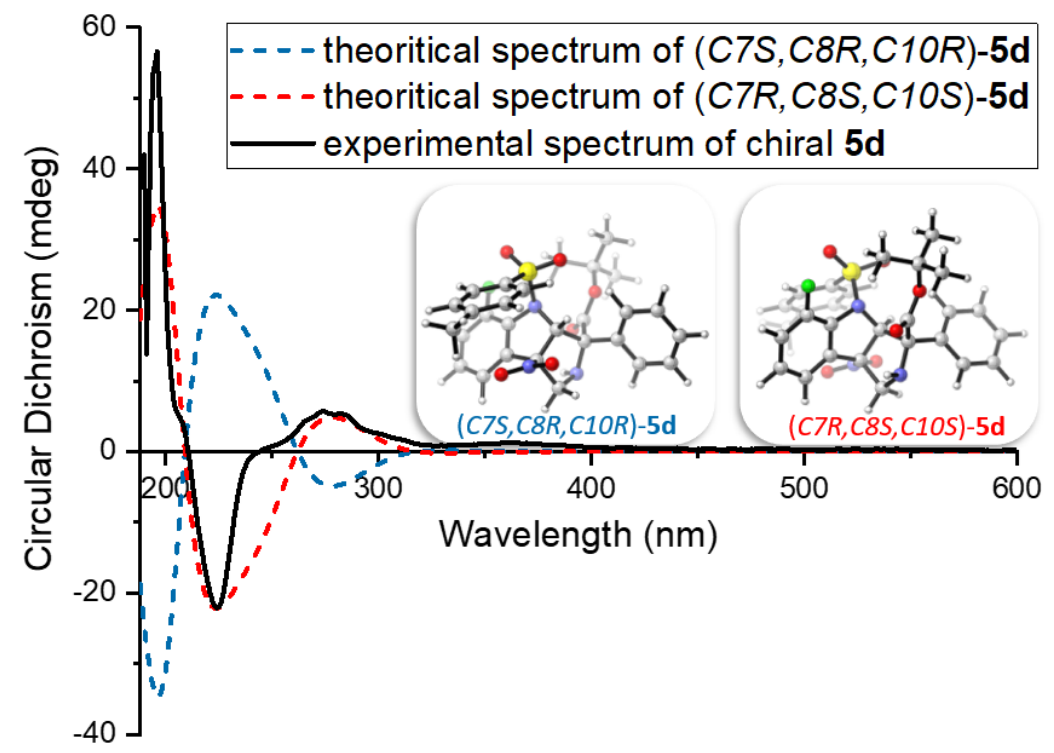

\section{Reference:}

1. Yanai, T.; Tew, D. P.; Handy, N. C. Chem. Phys. Lett. 2004, 393, 51-57.

2. Weigend, F.; Ahlrichs, R. Phys. Chem. Chem. Phys. 2005, 7, 3297-3305.

3. (a) Grimme, S.; Antony, J.; Ehrlich, S.; Krieg, H. J. Chem. Phys. 2010, 132, 154104; (b) Grimme, S.; Ehrlich, S.; Goerigk, L. J. Compt. Chem. 2011, 32, 1456-1465.

4. (a) Cancès, E.; Mennucci, B.; Tomasi, J. J. Chem. Phys. 1997, 107, 3032-3041. (b) Tomasi, J.; Mennucci, B.; Cancès, E. J. Mol. Struct. (Theochem) 1999, 464, 211-226.

5. Frisch, M. J.; Trucks, G. W.; Schlegel, H. B.; Scuseria, G. E.; Robb, M. A.; Cheeseman, J. R.; Scalmani, G.; Barone, V.; Mennucci, B.; Petersson, G. A.; Nakatsuji, H.; Caricato, M.; Li, X.; Hratchian, H. P.; Izmaylov, A. F.; Bloino, J.; Zheng, G.; Sonnenberg, J. L.; Hada, M.; Ehara, M.; Toyota, K.; Fukuda, R.; Hasegawa, J.; Ishida, M.; Nakajima, T.; Honda, Y.; Kitao, O.; Nakai, H.; Vreven, T.; Montgomery, Jr., J. A.; Peralta, J. E.; Ogliaro, F.; Bearpark, M.; Heyd, J. J.; Brothers, E.; Kudin, K. N.; Staroverov, V. N.; Kobayashi, R.; Normand, J.; Raghavachari, K.; Rendell, A.; Burant, J. C.; Iyengar, S. S.; Tomasi, J.; Cossi, M.; Rega, N.; Millam, J. M.; Klene, M.; Knox, J. E.; Cross, J. B.; Bakken, V.; Adamo, C.; Jaramillo, J.; Gomperts, R.; Stratmann, R. E.; Yazyev, O.; Austin, A. J.; Cammi, R.; Pomelli, C.; Ochterski, J. W.; Martin, R. L.; Morokuma, K.; Zakrzewski, V. G.; Voth, G. A.; Salvador, P.; Dannenberg, J. J.; Dapprich, S.; Daniels, A. D.; Farkas, Ö.; Foresman, J. B.; Ortiz, J. V.; Cioslowski, J.; Fox, D. J.; Gaussian, Inc.: Wallingford, CT, 2009.

\subsection{Cartesian coordinates for the optimized structures}

\section{$(C 7 S, C 8 R, C 10 R)-5 \mathrm{~d}$}

$\begin{array}{llll}\text { S } & -2.29452700 & -4.07033600 & -3.35477100 \\ \text { F } & -2.10326200 & -6.30158600 & -0.71358800 \\ \text { O } & -1.33873700 & -5.11416800 & -3.02881900 \\ \text { O } & -2.93580100 & -4.01849600 & -4.66199800 \\ \text { O } & -3.46403200 & -0.48506000 & -0.58289200 \\ \text { O } & -4.68380900 & -0.41659700 & -2.34842200 \\ \text { O } & -6.03361900 & -6.17968300 & -1.53610100 \\ \text { O } & -5.50514300 & -5.87312900 & -3.70114000 \\ \text { N } & -3.57271800 & -4.21891700 & -2.23421500\end{array}$




$\begin{array}{lrrr}\mathrm{N} & -6.72611600 & -3.63543500 & -1.18645700 \\ \mathrm{H} & -6.53731500 & -4.38252200 & -0.51990400 \\ \mathrm{~N} & -4.22526600 & -0.98201800 & -1.37873200 \\ \mathrm{C} & -3.67294500 & -2.98406500 & 1.18943700 \\ \mathrm{H} & -4.12700200 & -2.13646300 & 1.70263500 \\ \mathrm{C} & -2.91323300 & -3.91938600 & 1.88846900 \\ \mathrm{H} & -2.75851400 & -3.80395500 & 2.96204900 \\ \mathrm{C} & -2.37224800 & -5.02685500 & 1.23595700 \\ \mathrm{H} & -1.81145800 & -5.78827600 & 1.78002500 \\ \mathrm{C} & -2.56513200 & -5.20178500 & -0.12718600 \\ \mathrm{C} & -3.26517700 & -4.23929100 & -0.84892700 \\ \mathrm{C} & -3.83912400 & -3.15760100 & -0.17742200 \\ \mathrm{C} & -4.70855800 & -2.39412300 & -1.13475600 \\ \mathrm{C} & -4.67300600 & -3.26204800 & -2.41712300 \\ \mathrm{H} & -4.52264300 & -2.66284900 & -3.31618000 \\ \mathrm{C} & -6.08985000 & -3.95722200 & -2.45156200 \\ \mathrm{C} & -6.21205700 & -2.36194600 & -0.75794800 \\ \mathrm{H} & -6.68907100 & -1.54464900 & -1.31975200 \\ \mathrm{H} & -6.37217100 & -2.19592800 & 0.31399400 \\ \mathrm{C} & -5.88381500 & -5.48163500 & -2.51056800 \\ \mathrm{H} & -7.13016900 & -7.78993300 & -4.52065000 \\ \mathrm{C} & -6.91191200 & -3.46067900 & -3.64256600 \\ \mathrm{C} & -6.33887600 & -3.32310000 & -4.91165100 \\ \mathrm{H} & -5.28665800 & -3.56532000 & -5.06646100 \\ \mathrm{C} & -7.10838300 & -2.89500800 & -5.98852000 \\ \mathrm{H} & -6.64719500 & -2.79236900 & -6.97306300 \\ \mathrm{C} & -8.45970400 & -2.59872400 & -5.81460100 \\ \mathrm{H} & -9.06116400 & -2.25974900 & -6.66070200 \\ \mathrm{C} & -9.03503200 & -2.73701700 & -4.55516400 \\ \mathrm{H} & -10.09246000 & -2.50758600 & -4.40689800 \\ \mathrm{C} & -8.26491100 & -3.16749900 & -3.47603300 \\ \mathrm{H} & -8.70362400 & -3.27696200 & -2.48429000 \\ \mathrm{C} & -5.09603700 & -7.24917200 & -4.01090200 \\ \mathrm{C} & -3.95191400 & -7.67597900 & -3.10063200 \\ \mathrm{H} & -4.27054400 & -7.74268700 & -2.05460800 \\ \mathrm{H} & -3.59628900 & -8.66495500 & -3.42318300 \\ \mathrm{H} & -4.62364500 & -7.13524700 & -5.45160600 \\ \mathrm{H} & -8.21565500 & -2.84962700\end{array}$




\begin{tabular}{|c|c|c|c|}
\hline 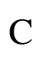 & 0 & 0 & \\
\hline & 1.95770900 & -1.38 & 0 \\
\hline & -2.60964600 & -1.51135000 & 4.62302800 \\
\hline & -1.48990700 & -0.13396800 & -3.38 \\
\hline & 4100 & 8200 & $-3.9^{\circ}$ \\
\hline & -0.65 & 5800 & -2.2 \\
\hline & -0.26 & 0400 & -1. \\
\hline & 0.40054400 & 8300 & \\
\hline & -0.72175400 & -2.37 & \\
\hline & -0.4 & 00 & -1. \\
\hline & -0.22 & 300 & 10 \\
\hline & & & \\
\hline & -0.131 & 2.08187300 & 1650 \\
\hline & -0.97659800 & 00 & 870 \\
\hline \multicolumn{4}{|c|}{$, C 8 S, C 10 S)-5 \mathrm{~d}$} \\
\hline 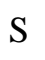 & -2.29452700 & -4.07033600 & -3.3 \\
\hline & -2.10 & 600 & 8800 \\
\hline & -1.33873700 & -5.11 & -3.02 \\
\hline & 100 & 500 & -4.6 \\
\hline & -3. & & \\
\hline & -4.68380900 & -0.41659700 & 4220 \\
\hline & -6.0 & -6.17 & -1.5 \\
\hline & & & \\
\hline & -3.5 & -4.2 & -2.2 \\
\hline & -6.72 & -3.63 & -1.1 \\
\hline & -6.53 & 00 & -0.5 \\
\hline & & 00 & \\
\hline & -3.67 & -2.98 & 370 \\
\hline & -4.12700200 & -2.13 & 350 \\
\hline & -2.91 & -3.9 & 690 \\
\hline & -2.75 & & \\
\hline & -2.37 & -5.0 & 570 \\
\hline $\mathrm{H}$ & 5800 & -5.78 & 250 \\
\hline & -2.56 & -5.20 & \\
\hline & -3.2 & -4.2 & -0.8 \\
\hline C & -3.83 & -3.1 & 20 \\
\hline & -4.70855800 & -2.39412300 & -1.1347560 \\
\hline & -4.67300600 & -3.26204800 & -2.4171230 \\
\hline & -4.52264300 & -2.66284900 & -3.3161800 \\
\hline & -6.08985000 & -3.95722200 & -2.4515620 \\
\hline & -6.21205700 & -2.36194600 & -0.7579480 \\
\hline 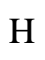 & -6.68907100 & -1.54464900 & -1.3197520 \\
\hline & -6.37217100 & -2.19592800 & 0.313994 \\
\hline C & -5.88381500 & -5.48163500 & -2.5105680 \\
\hline
\end{tabular}




$\begin{array}{lrrr}\mathrm{C} & -6.91191200 & -3.46067900 & -3.64256600 \\ \mathrm{C} & -6.33887600 & -3.32310000 & -4.91165100 \\ \mathrm{H} & -5.28665800 & -3.56532000 & -5.06646100 \\ \mathrm{C} & -7.10838300 & -2.89500800 & -5.98852000 \\ \mathrm{H} & -6.64719500 & -2.79236900 & -6.97306300 \\ \mathrm{C} & -8.45970400 & -2.59872400 & -5.81460100 \\ \mathrm{H} & -9.06116400 & -2.25974900 & -6.66070200 \\ \mathrm{C} & -9.03503200 & -2.73701700 & -4.55516400 \\ \mathrm{H} & -10.09246000 & -2.50758600 & -4.40689800 \\ \mathrm{C} & -8.26491100 & -3.16749900 & -3.47603300 \\ \mathrm{H} & -8.70362400 & -3.27696200 & -2.48429000 \\ \mathrm{C} & -5.09603700 & -7.24917200 & -4.01090200 \\ \mathrm{C} & -3.95191400 & -7.67597900 & -3.10063200 \\ \mathrm{H} & -4.27054400 & -7.74268700 & -2.05460800 \\ \mathrm{H} & -3.59628900 & -8.66495500 & -3.42318300 \\ \mathrm{H} & -3.11503500 & -6.96971700 & -3.16457700 \\ \mathrm{C} & -4.62364500 & -7.13524700 & -5.45160600 \\ \mathrm{H} & -3.79658900 & -6.41511300 & -5.51575000 \\ \mathrm{H} & -4.27922800 & -8.11356500 & -5.81398300 \\ \mathrm{H} & -5.44148600 & -6.78804900 & -6.09898000 \\ \mathrm{C} & -6.30975700 & -8.15929800 & -3.88846200 \\ \mathrm{H} & -7.13016900 & -7.78993300 & -4.52065000 \\ \mathrm{H} & -6.04402600 & -9.17140900 & -4.22511600 \\ \mathrm{H} & -6.65722600 & -8.21565500 & -2.84962700 \\ \mathrm{C} & -1.58417100 & -2.49463800 & -2.99889200 \\ \mathrm{C} & -1.95770900 & -1.38788000 & -3.75792700 \\ \mathrm{H} & -2.60964600 & -1.51135000 & -4.62302800 \\ \mathrm{C} & -1.48990700 & -0.13396800 & -3.38897400 \\ \mathrm{H} & -1.78604100 & 0.73958200 & -3.97281600 \\ \mathrm{C} & -0.65869600 & 0.02845800 & -2.27519800 \\ \mathrm{C} & -0.26714300 & -1.10840400 & -1.55696300 \\ \mathrm{H} & 0.40054400 & -1.00058300 & -0.69997100 \\ \mathrm{H} & -0.72175400 & -2.37055800 & -1.90856700 \\ \mathrm{H} & -0.22464600 & 1.39709300 & -1.83941100 \\ \mathrm{H} & -73271500 & 1.36632600 & -1.30206900 \\ \mathrm{H} & -13118200 & 2.08187300 & -2.69316500 \\ \mathrm{H} & -1.15508700\end{array}$


14. NMR Spectra and HPLC

${ }^{1} \mathrm{H}$ NMR, ${ }^{13} \mathrm{C}$ NMR and HPLC of $3 \mathrm{a}$

(300 MHz, $\mathrm{CDCl}_{3},{ }^{1} \mathrm{H}$ NMR)
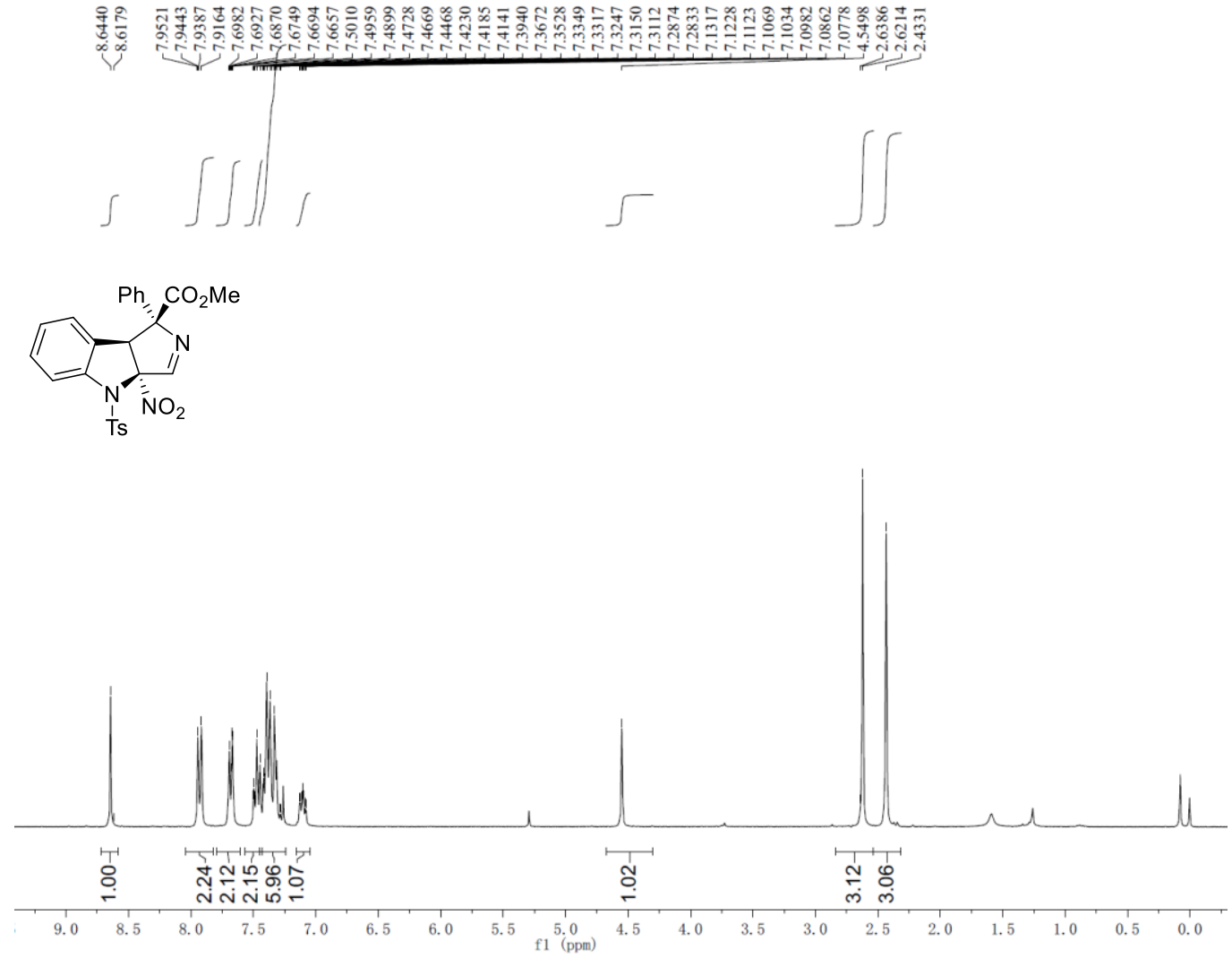

(75 MHz, $\mathrm{CDCl}_{3},{ }^{13} \mathrm{C}$ NMR)
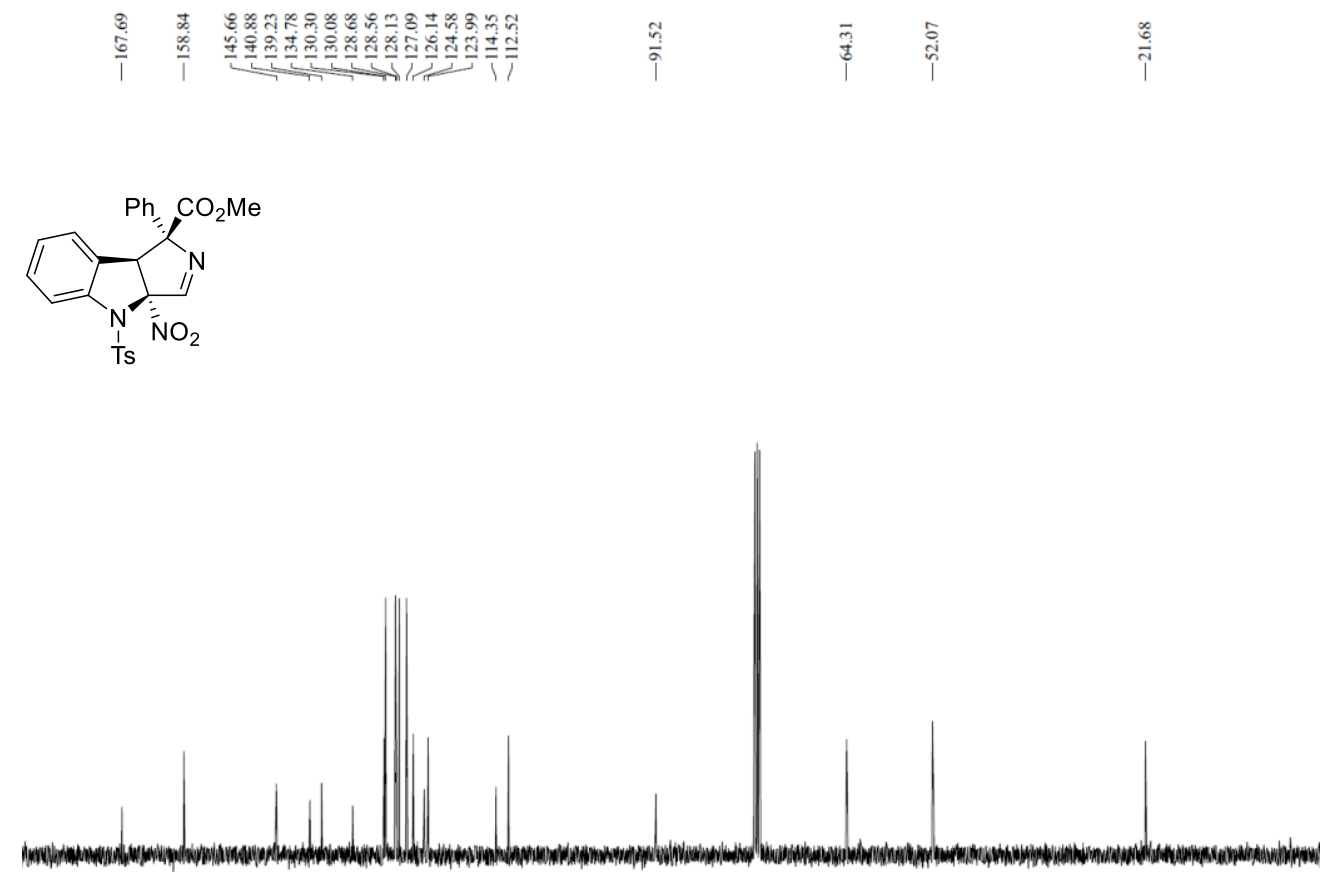

$\begin{array}{llllllllll}180 & 170 & 160 & 150 & 140 & 130 & 120 & 110 & 100 & 1\end{array}$ 


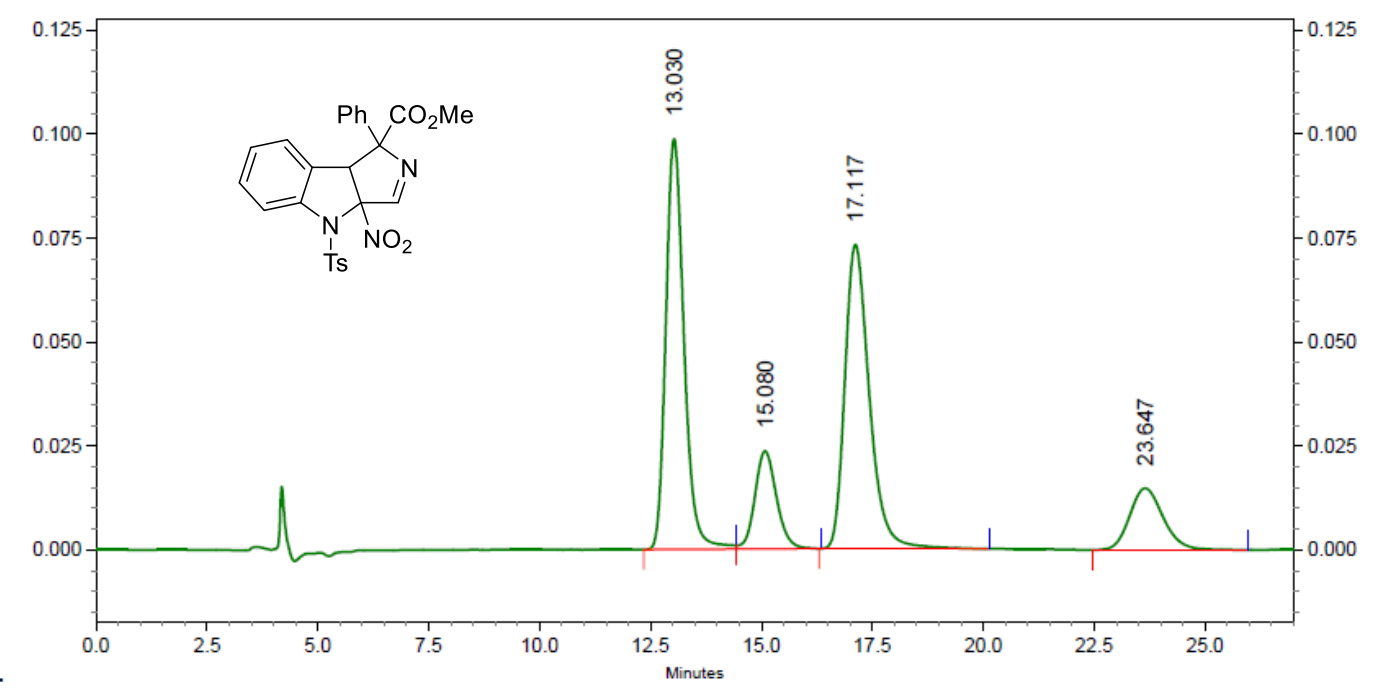

Detector

\begin{tabular}{rrrrrr}
$\begin{array}{r}\text { A (254nm) } \\
\text { Pk \# }\end{array}$ & Retention Time & Height & $\begin{array}{r}\text { Height } \\
\text { Percent }\end{array}$ & Area & Area Percent \\
\hline 1 & 13.030 & 98655 & 46.99 & 2806832 & 39.12 \\
2 & 15.080 & 23501 & 11.19 & 795230 & 11.08 \\
3 & 17.117 & 73036 & 34.78 & 2802630 & 39.07 \\
4 & 23.647 & 14773 & 7.04 & 769482 & 10.73
\end{tabular}

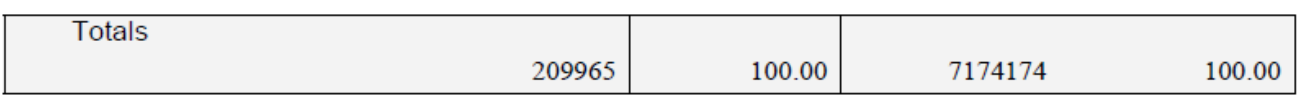

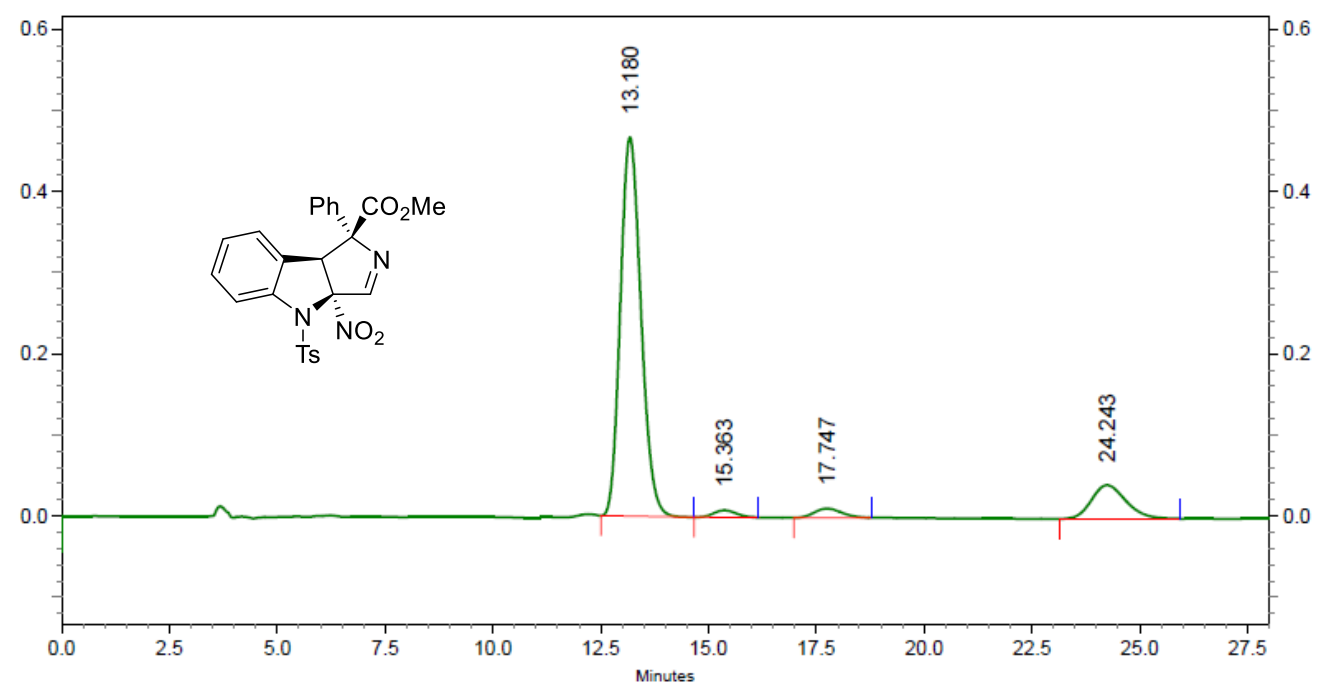

Detector

\begin{tabular}{rrrrrr}
$\begin{array}{r}\text { A (254nm) } \\
\text { Pk \# }\end{array}$ & Retention Time & Height & $\begin{array}{r}\text { Height } \\
\text { Percent }\end{array}$ & Area & Area Percent \\
\hline 1 & 13.180 & 467045 & 88.39 & 15528298 & 83.34 \\
2 & 15.363 & 8666 & 1.64 & 318937 & 1.71 \\
3 & 17.747 & 11326 & 2.14 & 500875 & 2.69 \\
4 & 24.243 & 41356 & 7.83 & 2283552 & 12.26 \\
\hline \multicolumn{7}{r}{} & 528393 & 100.00 & 18631662 & 100.00 \\
\hline
\end{tabular}


${ }^{1} \mathrm{H}$ NMR, ${ }^{13} \mathrm{C}$ NMR and HPLC of $3 b$

(300 MHz, $\mathrm{CDCl}_{3},{ }^{1} \mathrm{H}$ NMR)

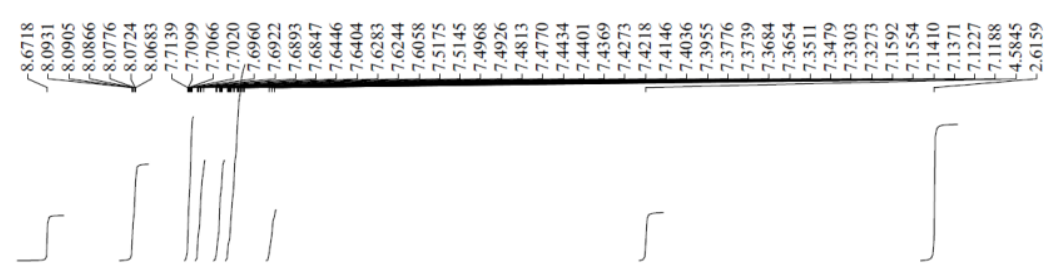

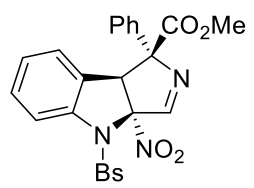

(75 $\left.\mathrm{MHz}, \mathrm{CDCl}_{3},{ }^{13} \mathrm{C} \mathrm{NMR}\right)$

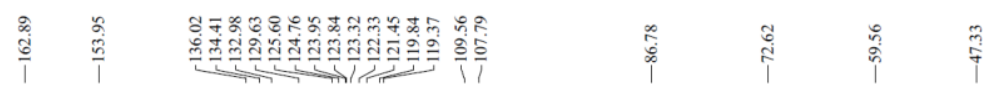
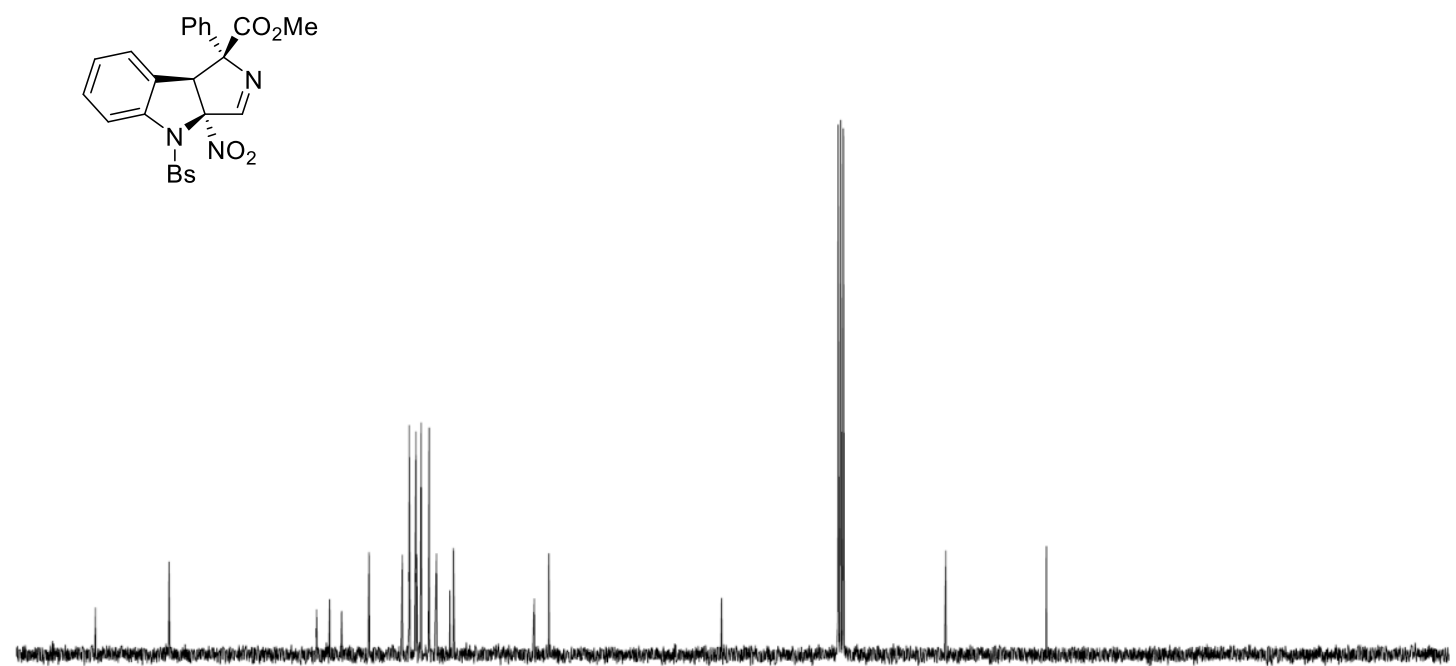

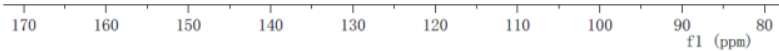




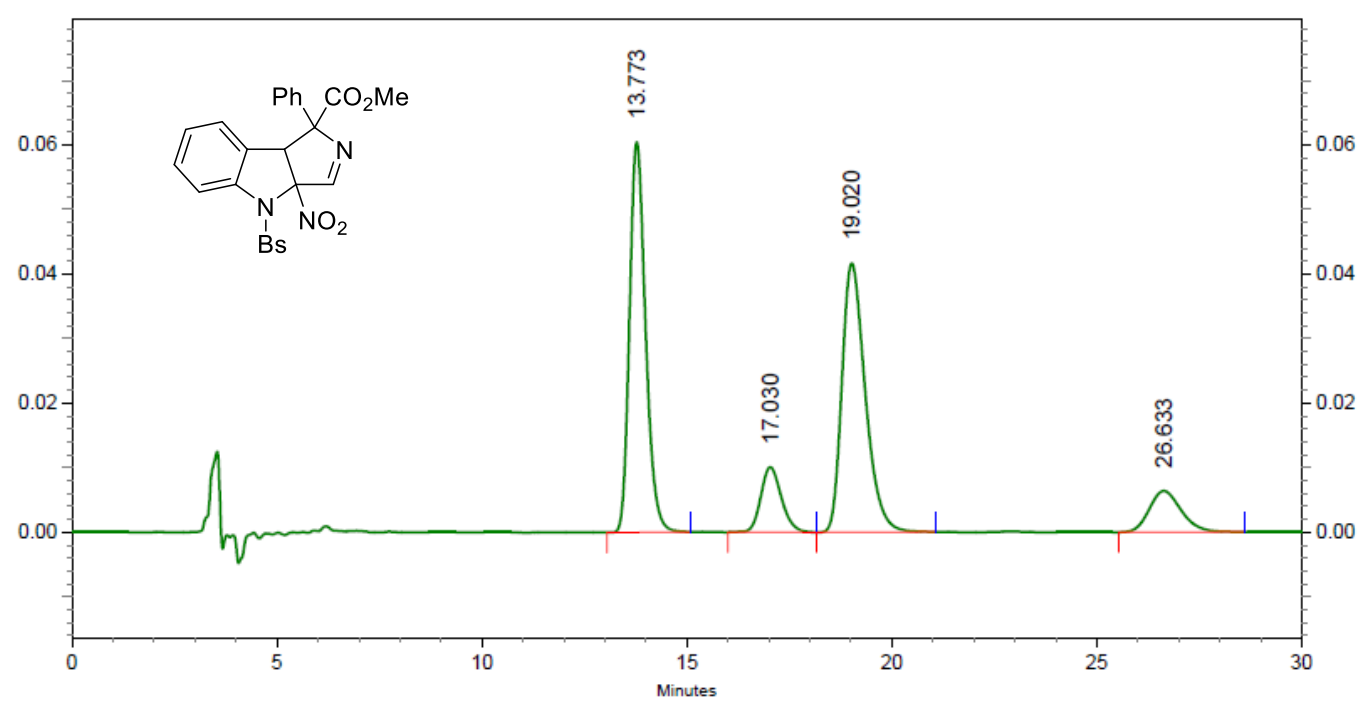

Detector

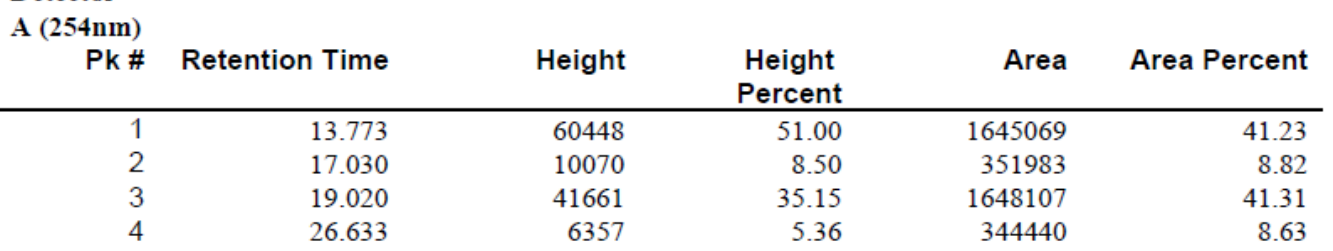

\begin{tabular}{|c|c|c|c|c|}
\hline Totals & & & & \\
\hline & 118536 & 100.00 & 3989599 & 100.00 \\
\hline
\end{tabular}

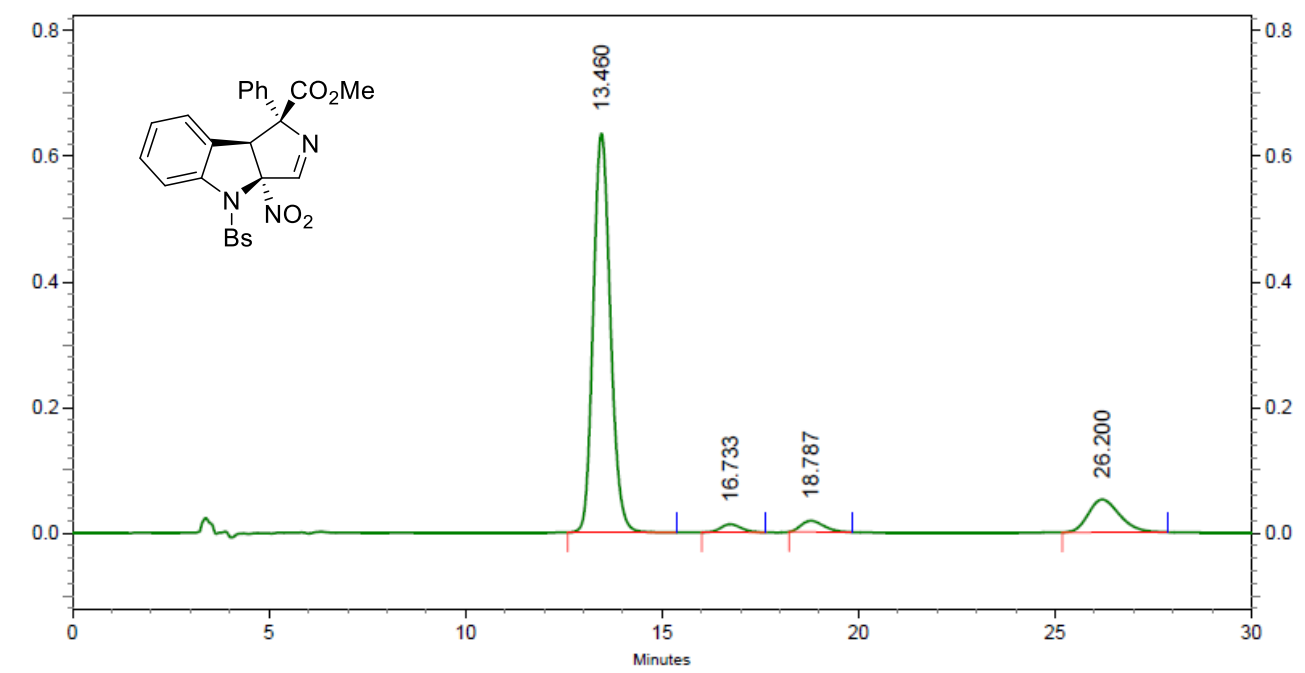

Detector

\begin{tabular}{|c|c|c|c|c|c|}
\hline \multicolumn{6}{|l|}{$A(254 \mathrm{~nm})$} \\
\hline $\mathrm{Pk} \#$ & Retention Time & Height & $\begin{array}{l}\text { Height } \\
\text { Percent }\end{array}$ & Area & Area Percent \\
\hline 1 & 13.460 & 635242 & 88.46 & 19285283 & 82.55 \\
\hline 2 & 16.733 & 12896 & 1.80 & 465409 & 1.99 \\
\hline 3 & 18.787 & 17820 & 2.48 & 720843 & 3.09 \\
\hline 4 & 26.200 & 52172 & 7.26 & 2890540 & 12.37 \\
\hline
\end{tabular}

\begin{tabular}{|l|r|r|rr|}
\hline Totals & & & & \\
& 718130 & 100.00 & 23362075 & 100.00 \\
\hline
\end{tabular}


${ }^{1} \mathrm{H}$ NMR, ${ }^{13} \mathrm{C}$ NMR and HPLC of $3 \mathrm{c}$

(300 $\mathrm{MHz}, \mathrm{CDCl}_{3},{ }^{1} \mathrm{H}$ NMR)
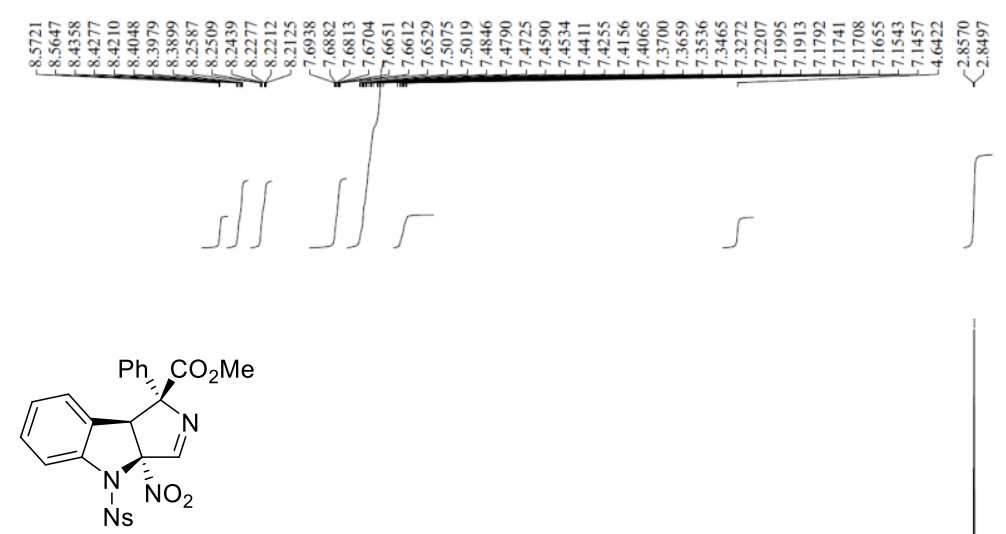

(75 MHz, $\left.\mathrm{CDCl}_{3},{ }^{13} \mathrm{C} \mathrm{NMR}\right)$
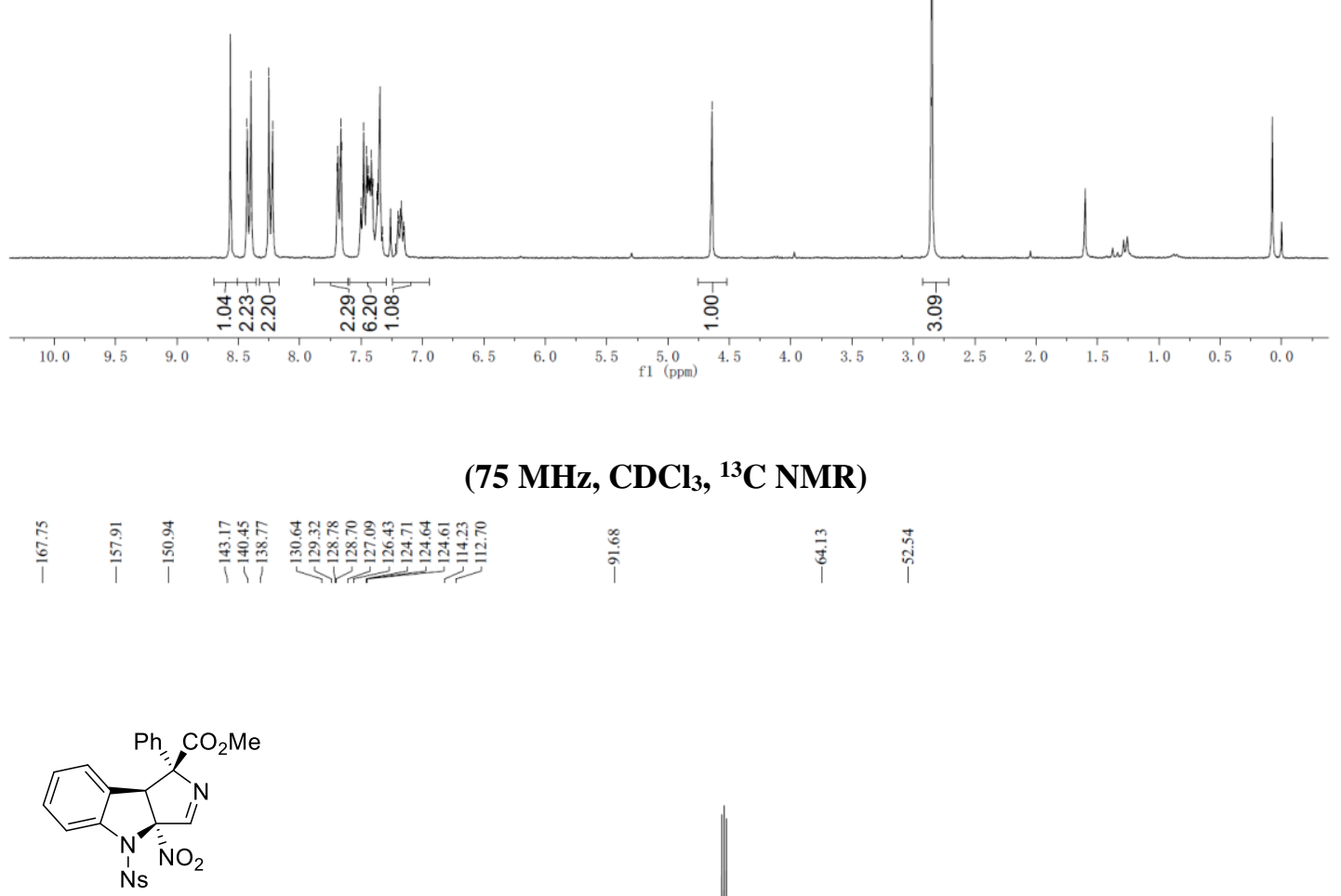

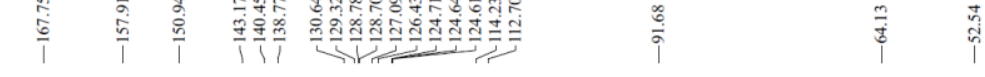

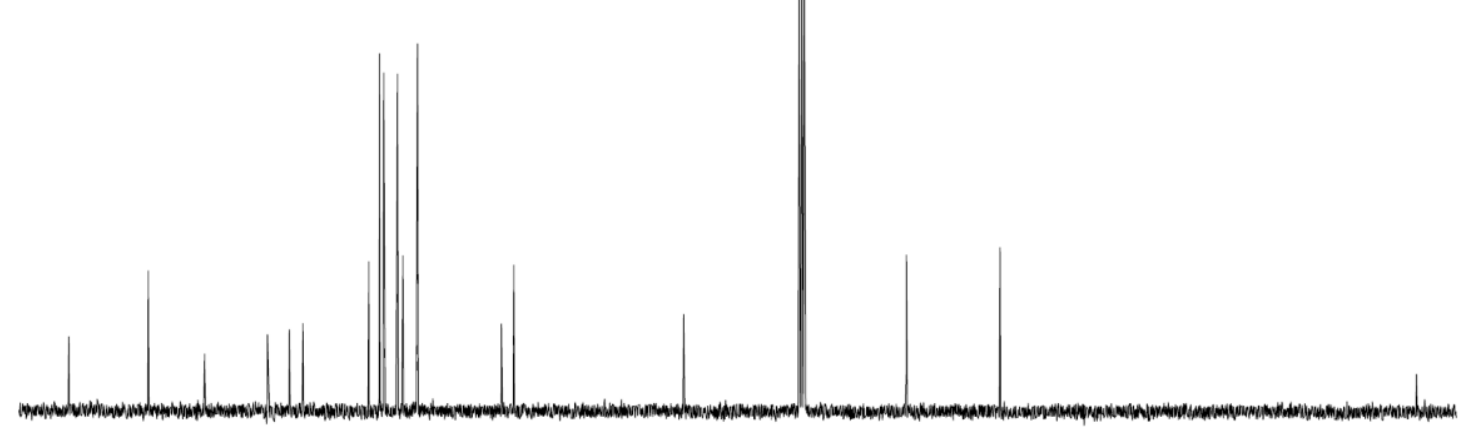

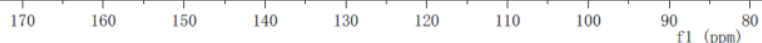




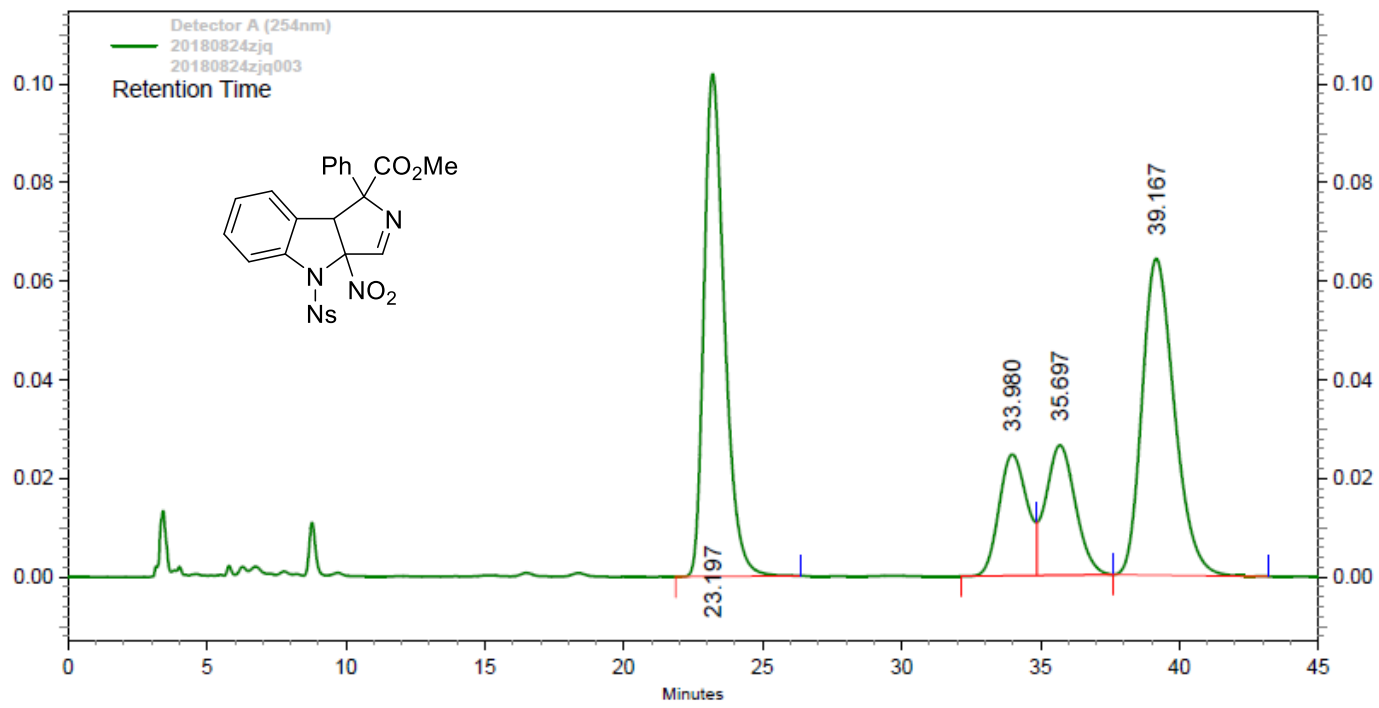

Detector

A (254nm)

Pk \# Retention Time 123.197

33.980

Height Height Percent

Area

Area Percent

$\begin{array}{ll}2 & 33.980 \\ 3 & 35.697\end{array}$

101962

46.97

11.32

5233532

37.09

39.167

26351

12.14

1957418

12.27

64181

29.57

5186234

36.76

\begin{tabular}{|c|c|c|c|c|}
\hline \multirow[t]{2}{*}{ Totals } & & & & \\
\hline & 217069 & 100.00 & 14108800 & 100.00 \\
\hline
\end{tabular}

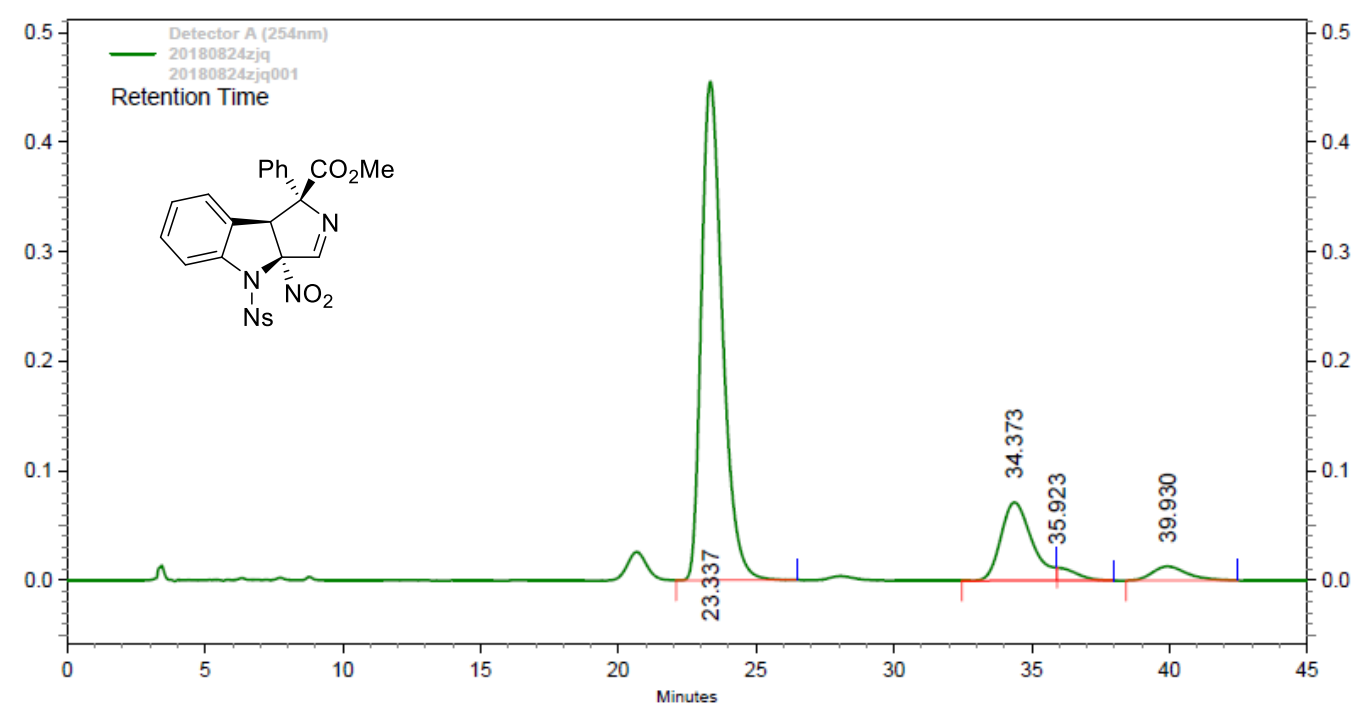

Detector

A (254nm)

\begin{tabular}{rrrrrr} 
Pk \# & Retention Time & Height & Height Percent & Area & Area Percent \\
\hline 1 & 23.337 & 455417 & 82.57 & 24201804 & 76.76 \\
2 & 34.373 & 71463 & 12.96 & 5620816 & 17.83 \\
3 & 35.923 & 11813 & 2.14 & 565498 & 1.79 \\
4 & 39.930 & 12871 & 2.33 & 1140562 & 3.62
\end{tabular}

Totals

551564

100.00

31528680

100.00 
${ }^{1} \mathrm{H}$ NMR, ${ }^{13} \mathrm{C}$ NMR and HPLC of $3 \mathrm{~d}$

(300 $\mathrm{MHz}, \mathrm{CDCl}_{3},{ }^{1} \mathrm{H}$ NMR)

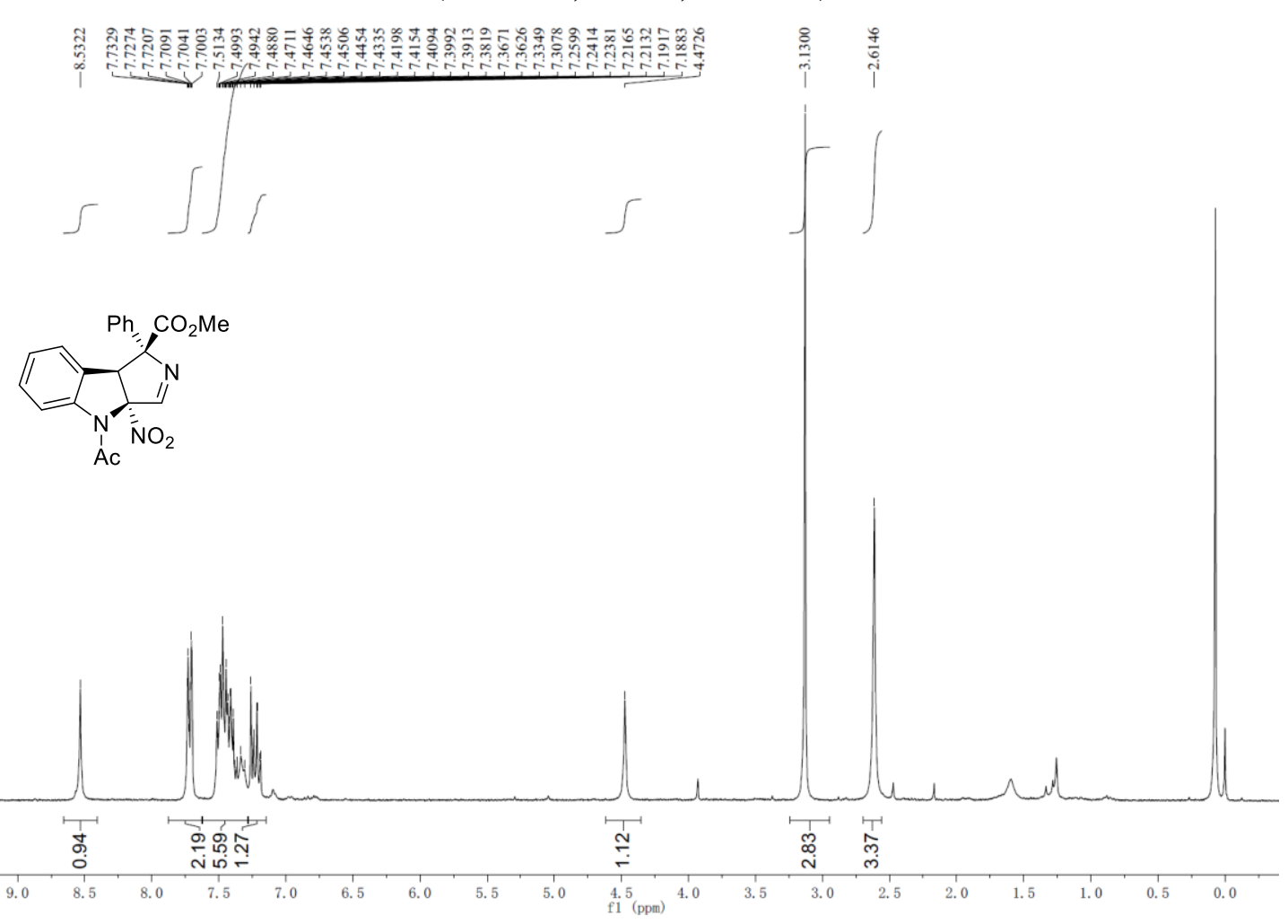

(75 $\left.\mathrm{MHz}, \mathrm{CDCl}_{3},{ }^{13} \mathrm{C} \mathrm{NMR}\right)$
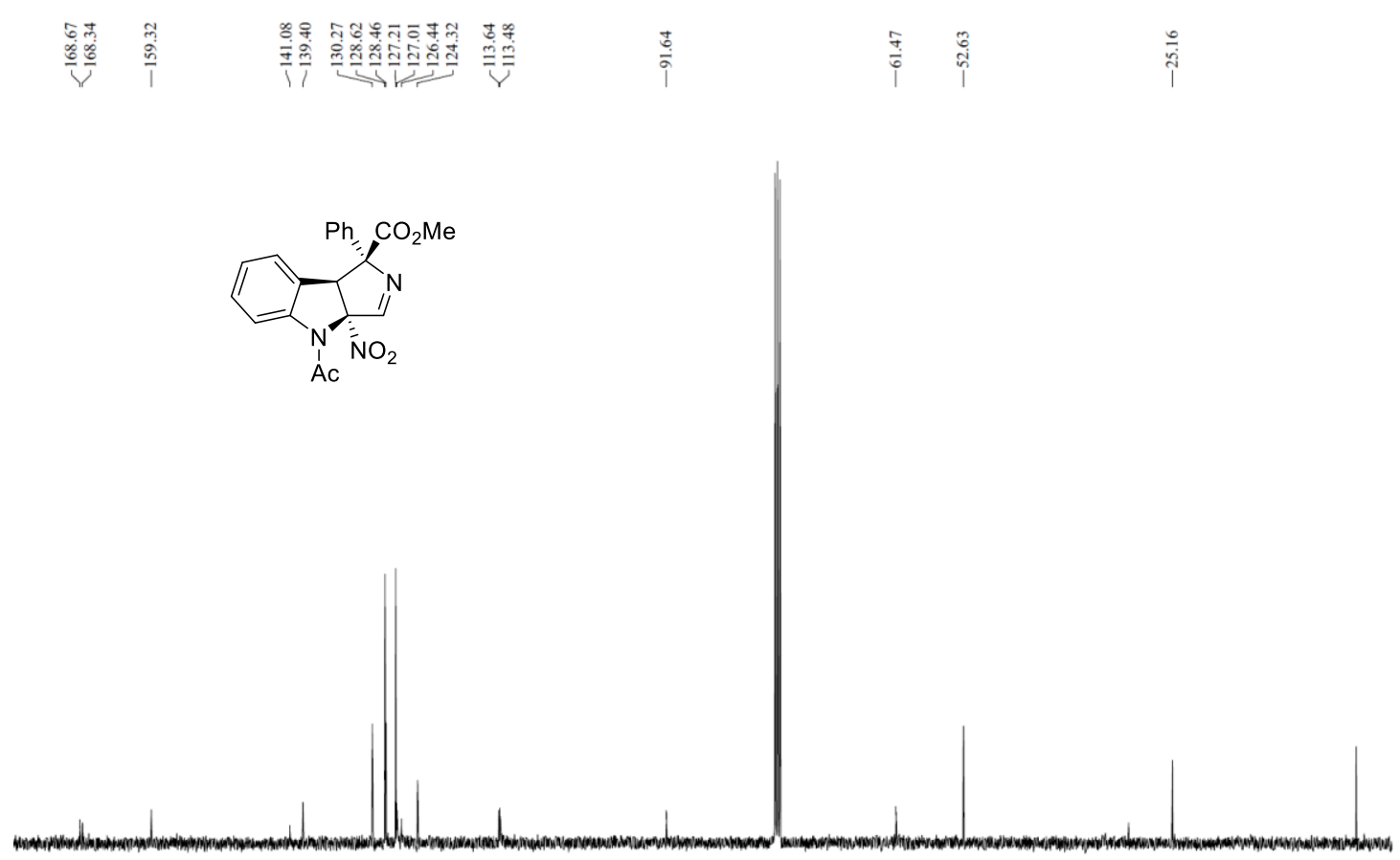

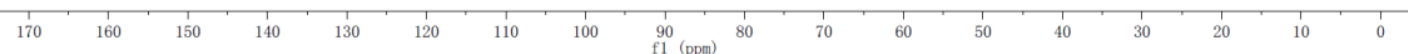




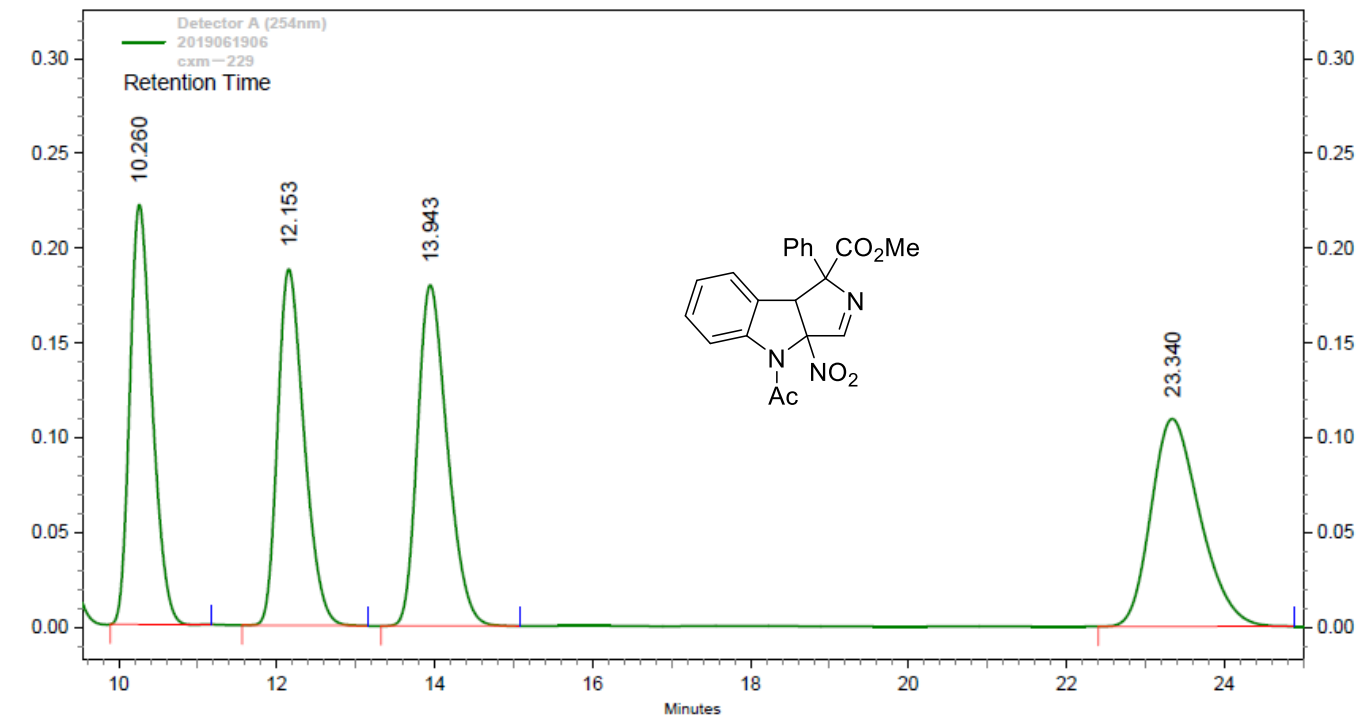

Detector

\begin{tabular}{|c|c|c|c|c|c|}
\hline $\begin{array}{r}A(254 n m) \\
P k \#\end{array}$ & Retention Time & Height & $\begin{array}{l}\text { Height } \\
\text { Percent }\end{array}$ & Area & Area Percent \\
\hline 1 & 10.260 & 221476 & 31.69 & 4334691 & 23.86 \\
\hline 2 & 12.153 & 188018 & 26.90 & 4380101 & 24.11 \\
\hline 3 & 13.943 & 179840 & 25.73 & 4720882 & 25.99 \\
\hline 4 & 23.340 & 109575 & 15.68 & 4731588 & 26.04 \\
\hline
\end{tabular}

\begin{tabular}{|l|r|r|rr|}
\hline Totals & 698909 & 100.00 & 18167262 & 100.00 \\
\hline
\end{tabular}

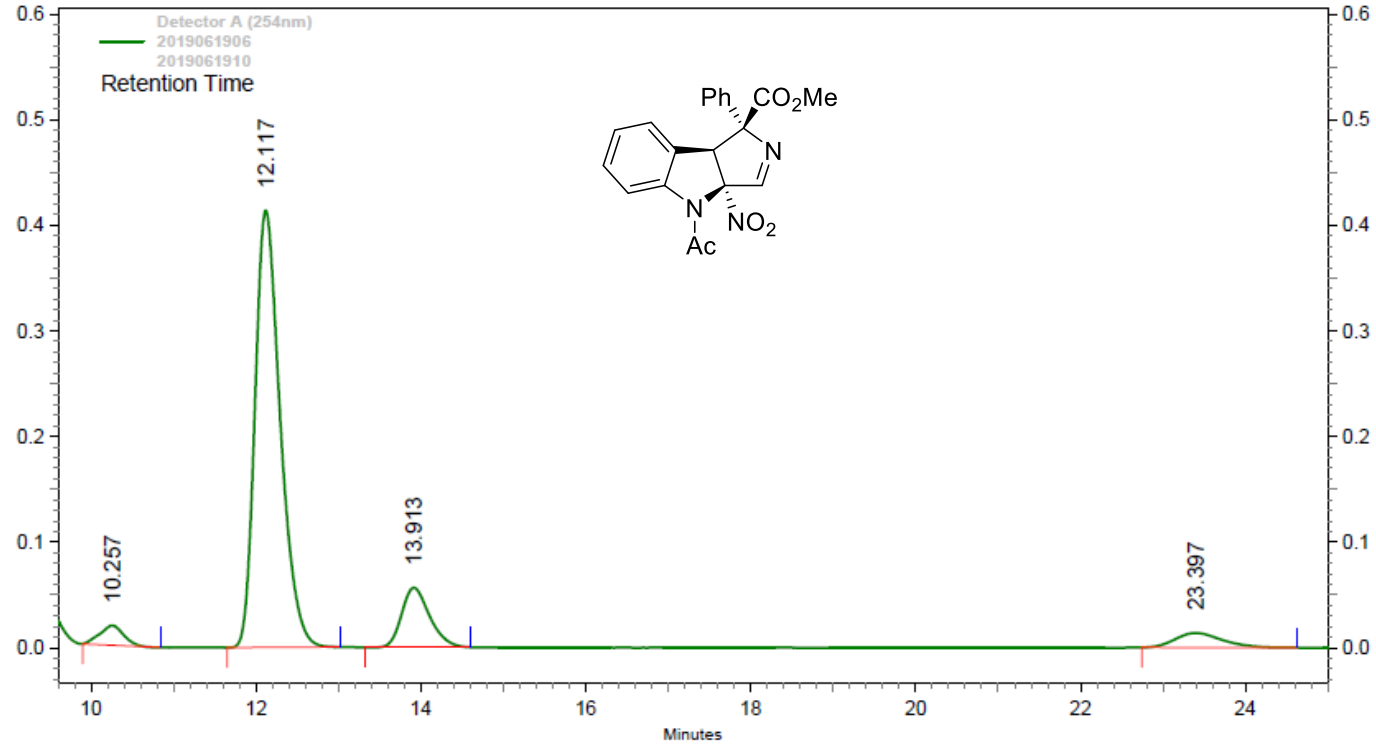

Detector

A (254nm)

\begin{tabular}{rrrrrr} 
Pk \# & Retention Time & Height & $\begin{array}{c}\text { Height } \\
\text { Percent }\end{array}$ & Area & Area Percent \\
\hline 1 & 10.257 & 18628 & 3.71 & 373399 & 3.38 \\
2 & 12.117 & 413690 & 82.32 & 8784288 & 79.60 \\
3 & 13.913 & 56413 & 11.23 & 1328088 & 12.04 \\
4 & 23.397 & 13810 & 2.75 & 549353 & 4.98
\end{tabular}

\begin{tabular}{|c|c|c|c|c|}
\hline Totals & & & & \\
\hline & 502541 & 100.00 & 11035128 & 100.00 \\
\hline
\end{tabular}


${ }^{1} \mathrm{H}$ NMR, ${ }^{13} \mathrm{C}$ NMR and HPLC of 3e (major)

(300 $\mathrm{MHz}, \mathrm{CDCl}_{3},{ }^{1} \mathrm{H} \mathrm{NMR}$ )

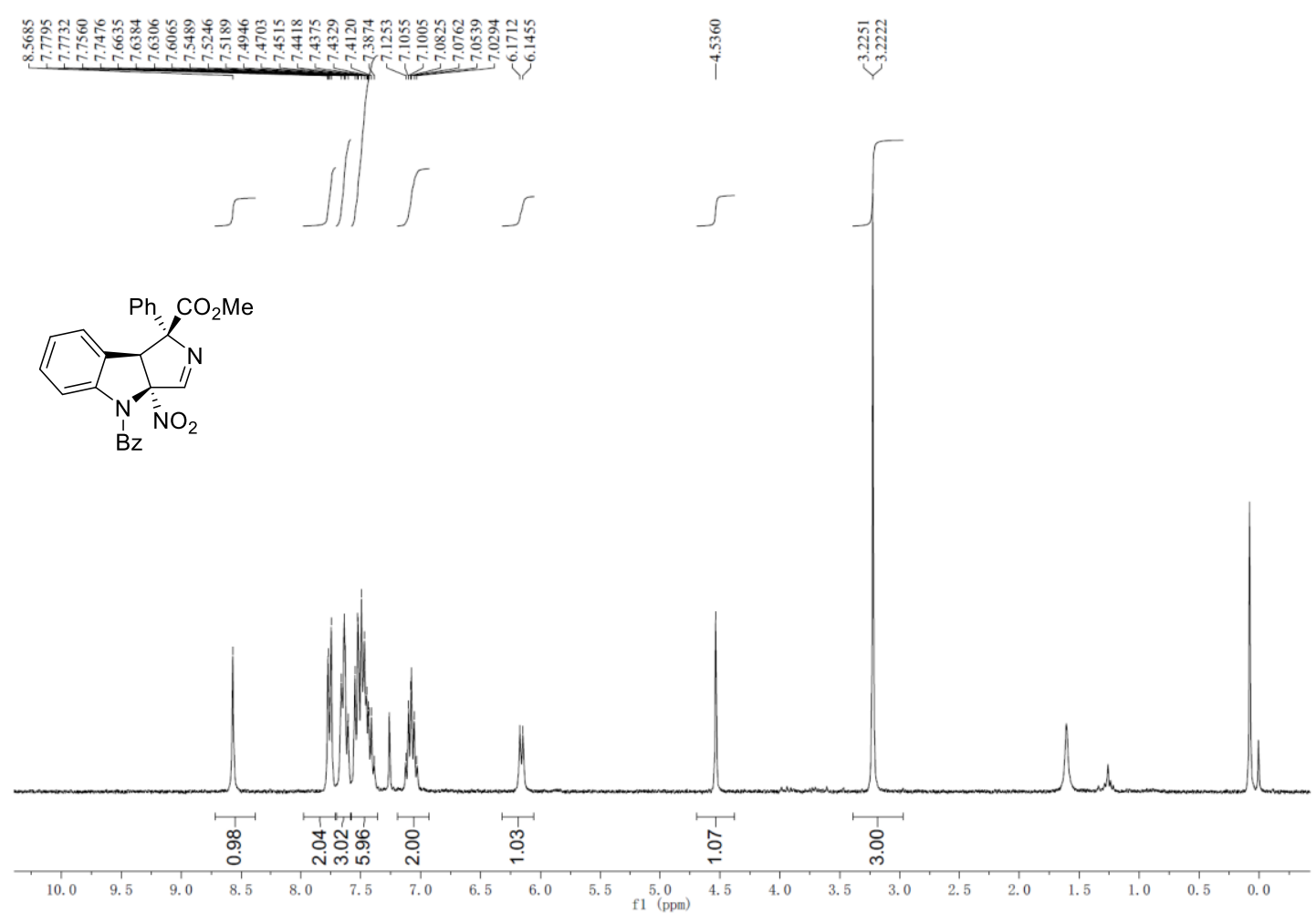

$\left(75 \mathrm{MHz}, \mathrm{CDCl}_{3},{ }^{13} \mathrm{C} \mathrm{NMR}\right)$

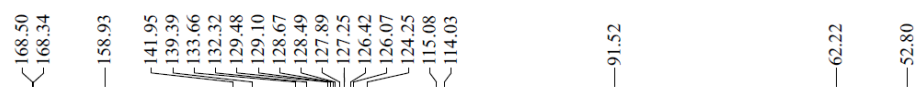
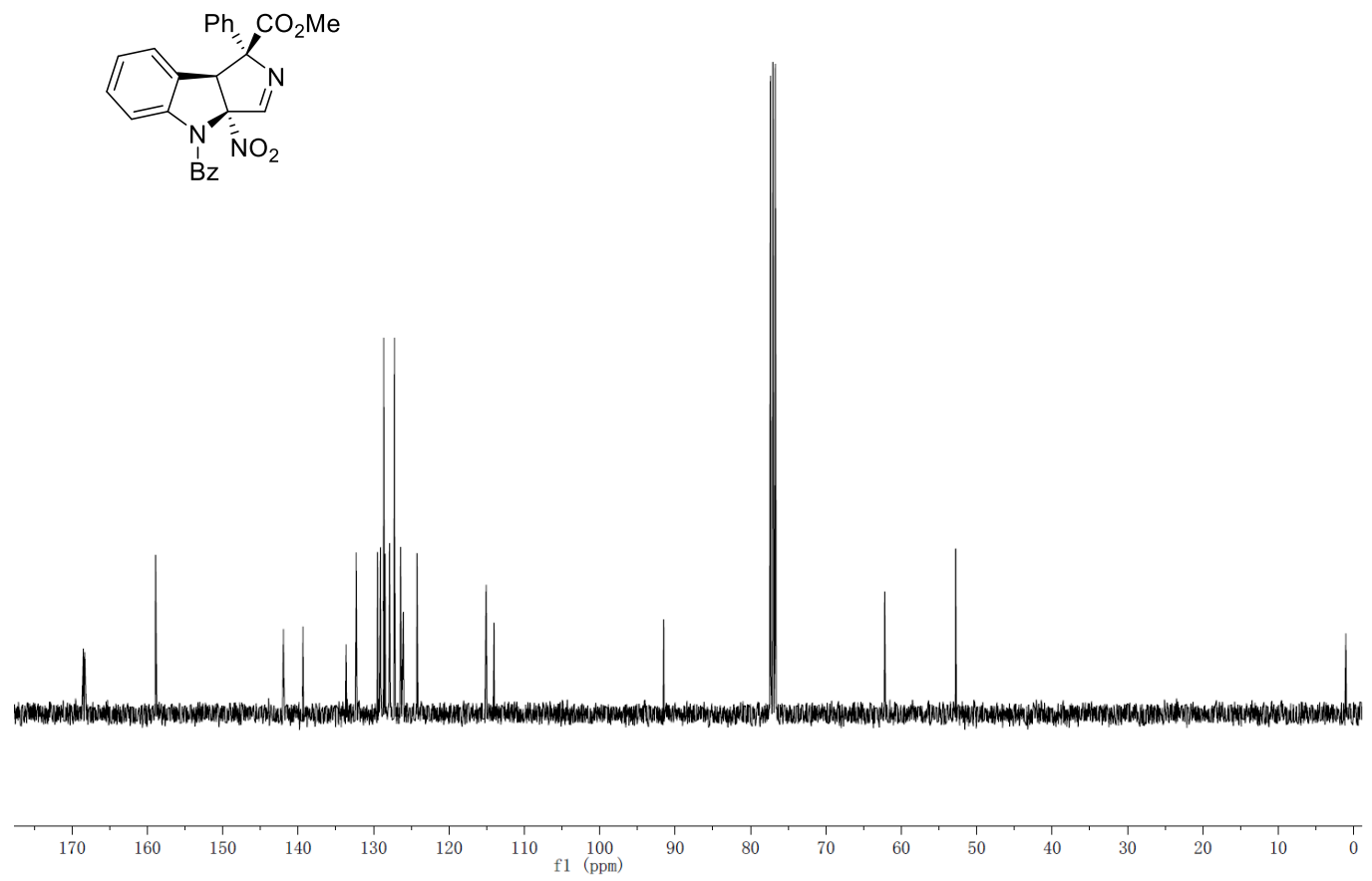
${ }^{1} \mathrm{H}$ NMR, ${ }^{13} \mathrm{C}$ NMR and HPLC of $3 \mathrm{e}$ ( minor)

(300 $\mathrm{MHz}, \mathrm{CDCl}_{3},{ }^{1} \mathrm{H}$ NMR)
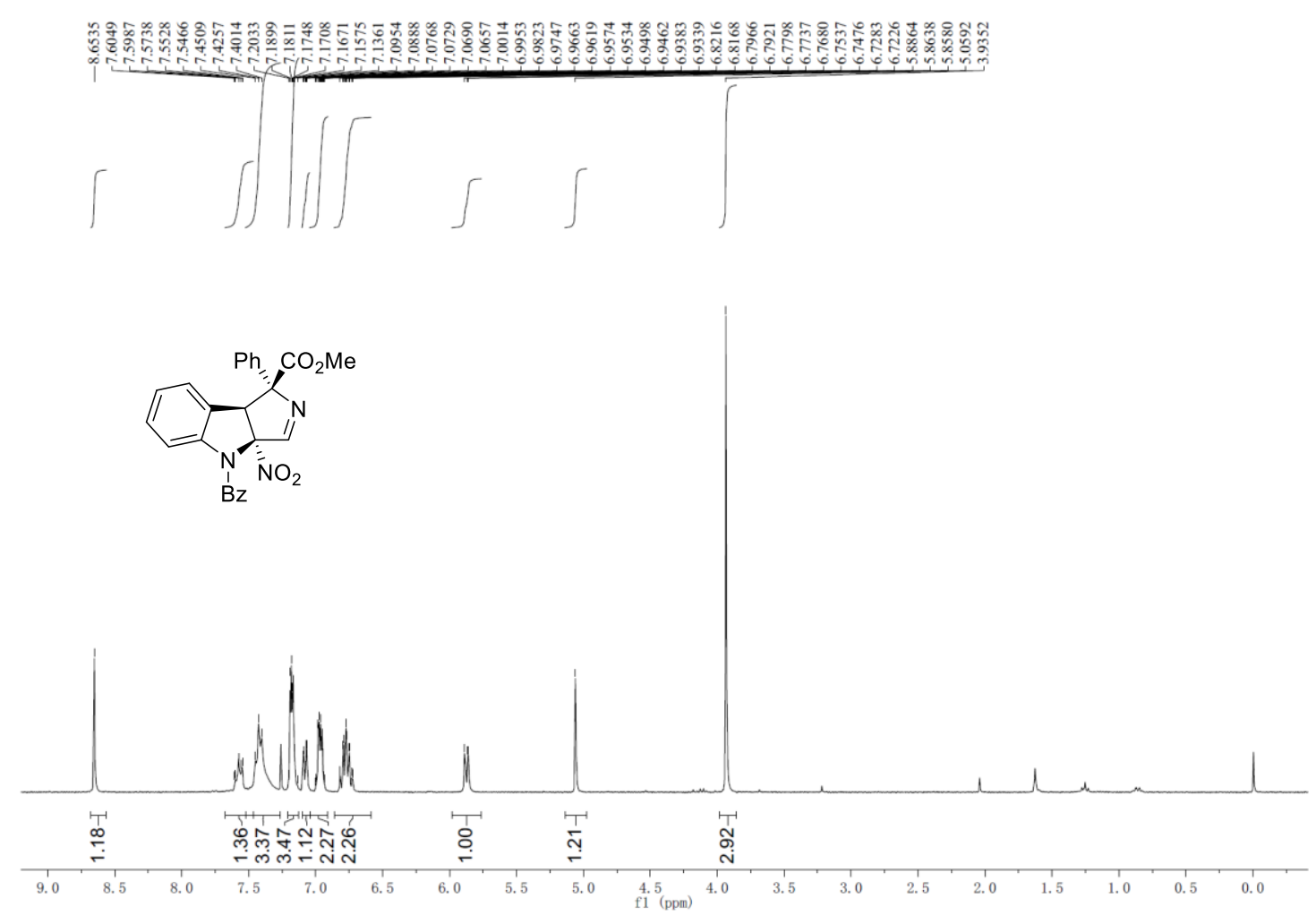

$\left(75 \mathrm{MHz}, \mathrm{CDCl}_{3},{ }^{13} \mathrm{C} \mathrm{NMR}\right)$
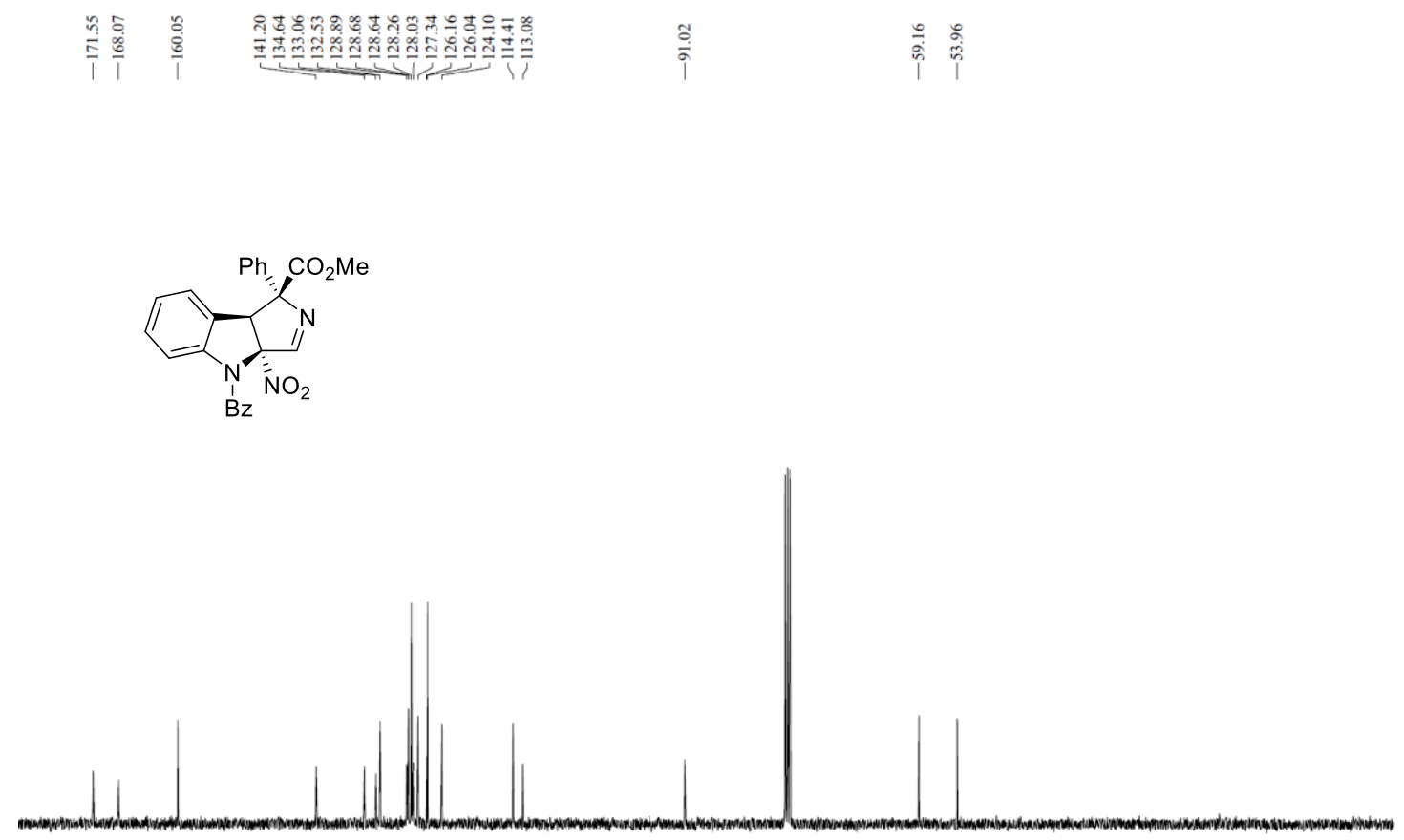

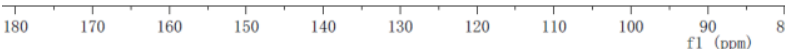




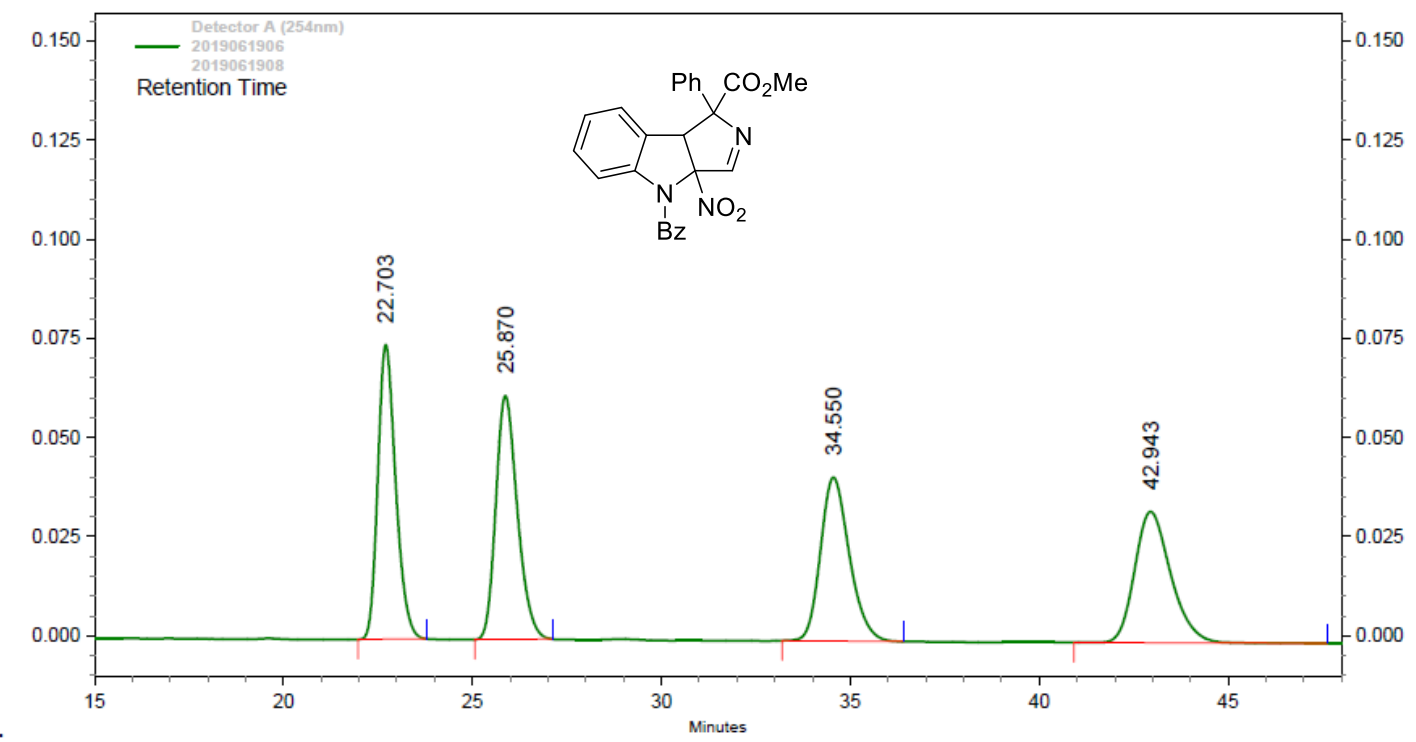

Detector

\begin{tabular}{rrrrrr}
$\begin{array}{r}\text { A (254nm) } \\
\text { Pk \# }\end{array}$ & Retention Time & Height & $\begin{array}{c}\text { Height } \\
\text { Percent }\end{array}$ & Area & Area Percent \\
\hline 1 & 22.703 & 74133 & 35.34 & 2441605 & 26.33 \\
2 & 25.870 & 61365 & 29.25 & 2440662 & 26.32 \\
3 & 34.550 & 41229 & 19.66 & 2193126 & 23.65 \\
4 & 42.943 & 33033 & 15.75 & 2198478 & 23.71 \\
\hline \multicolumn{2}{|c|}{209760} & 100.00 & 9273871 & 100.00 \\
\hline
\end{tabular}

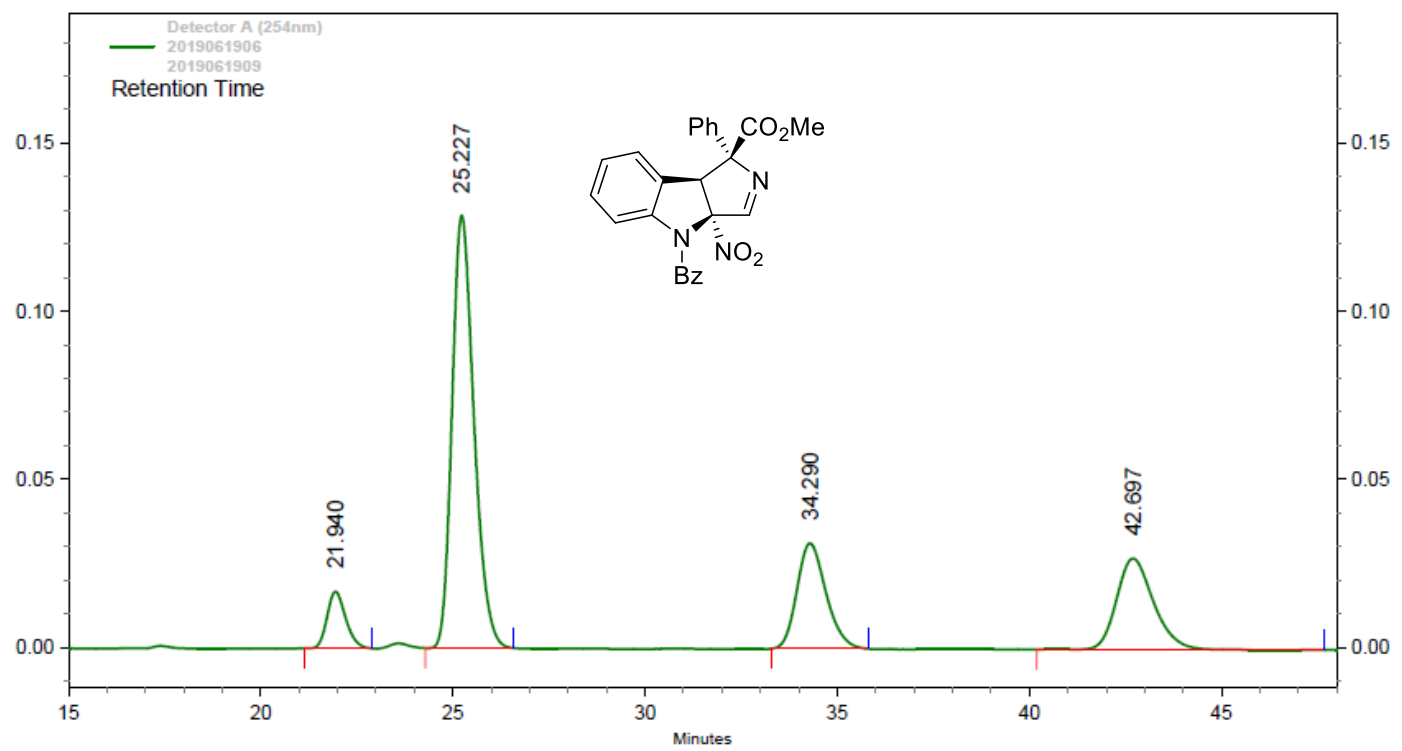

Detector

A (254nm)

Pk \# Retention Time

\begin{tabular}{rrrrr} 
Height & $\begin{array}{c}\text { Height } \\
\text { Percent }\end{array}$ & Area & Area Percent \\
\hline 70 & 16869 & 8.27 & 567770 & 6.23 \\
77 & 128683 & 63.08 & 5094759 & 55.86 \\
67 & 31340 & 15.36 & 1649178 & 18.08 \\
& 27111 & 13.29 & 1809011 & 19.83
\end{tabular}

Totals 
${ }^{1} \mathrm{H}$ NMR, ${ }^{13} \mathrm{C}$ NMR and HPLC of $3 f$

(300 $\mathrm{MHz}, \mathrm{CDCl}_{3},{ }^{1} \mathrm{H}$ NMR)
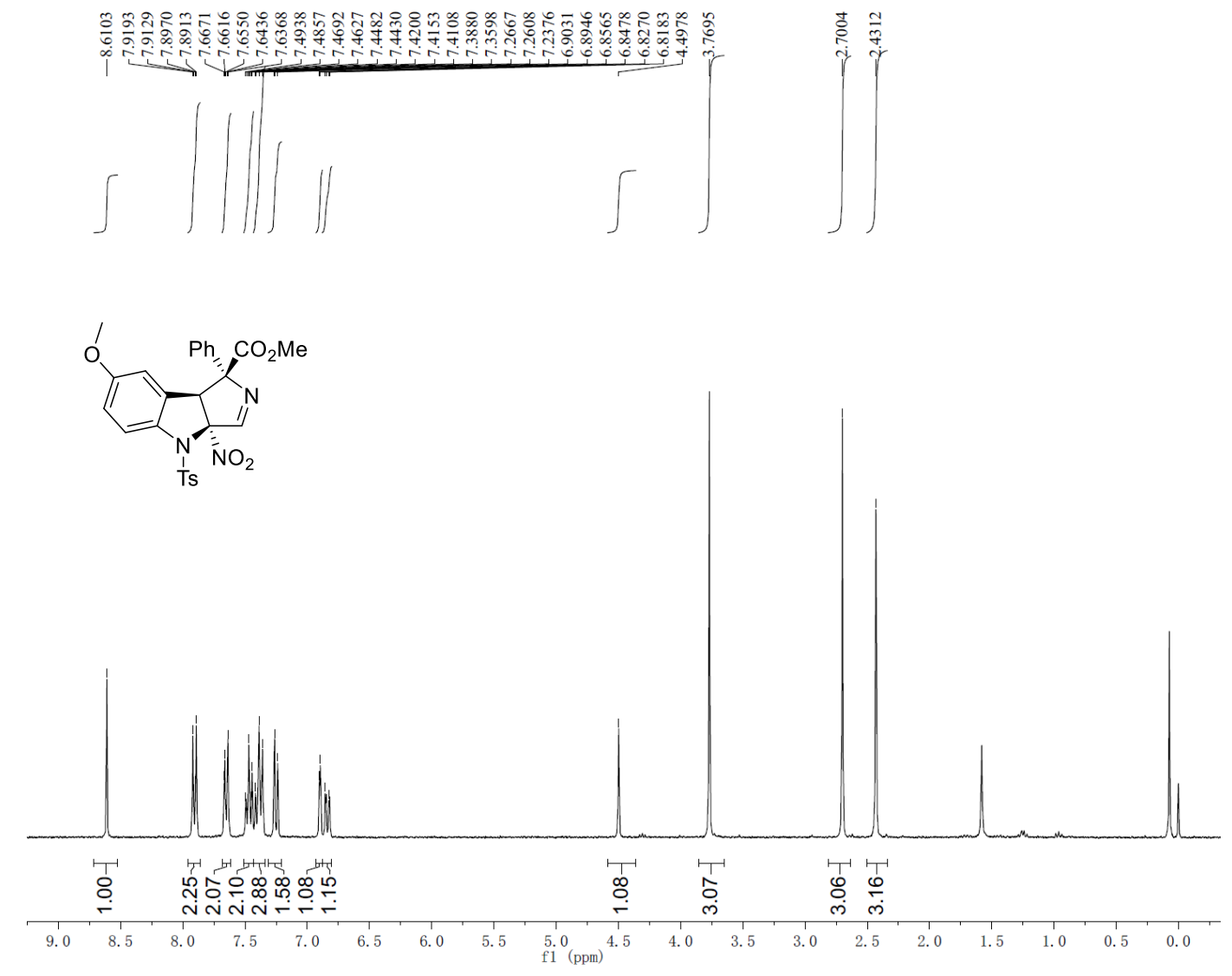

(75 MHz, $\left.\mathrm{CDCl}_{3},{ }^{13} \mathrm{C} \mathrm{NMR}\right)$

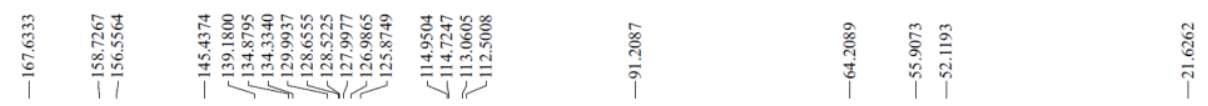

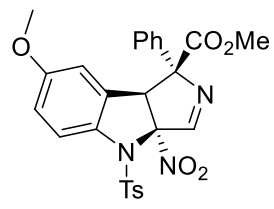

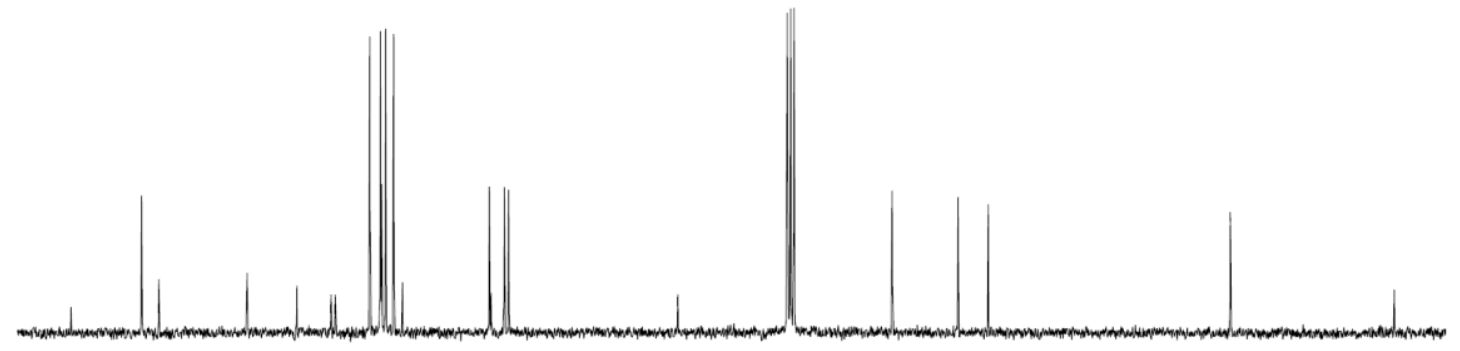

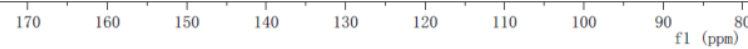




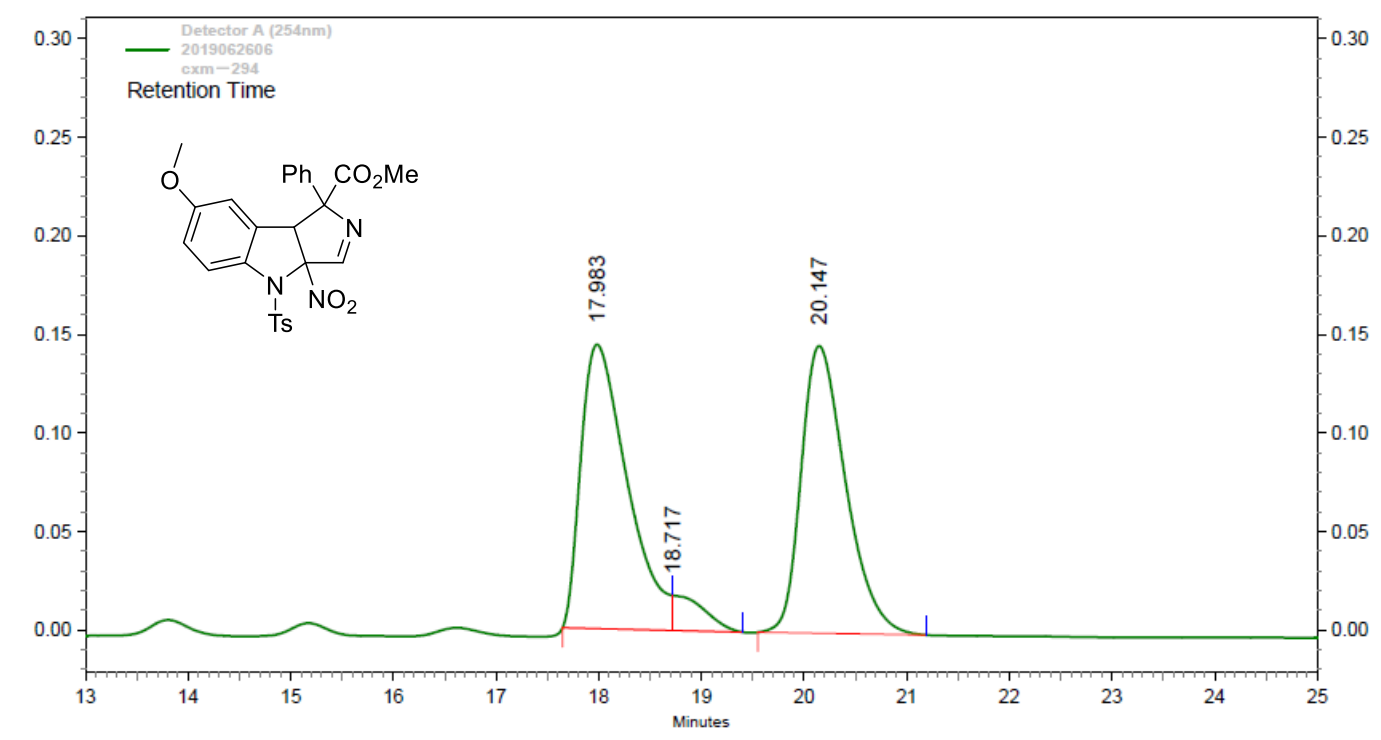

Detector

\begin{tabular}{|c|c|c|c|c|c|}
\hline $\begin{array}{r}\text { A (254nm) } \\
\text { Pk \# }\end{array}$ & Retention Time & Height & $\begin{array}{l}\text { Height } \\
\text { Percent }\end{array}$ & Area & Area Percent \\
\hline 1 & 17.983 & 144098 & 46.84 & 4528547 & 48.64 \\
\hline 2 & 18.717 & 17798 & 5.79 & 383181 & 4.12 \\
\hline 3 & 20.147 & 145752 & 47.38 & 4398304 & 47.24 \\
\hline
\end{tabular}

\begin{tabular}{|c|c|c|c|c|}
\hline \multirow[t]{2}{*}{ Totals } & & & & \\
\hline & 307648 & 100.00 & 9310032 & 100.00 \\
\hline
\end{tabular}

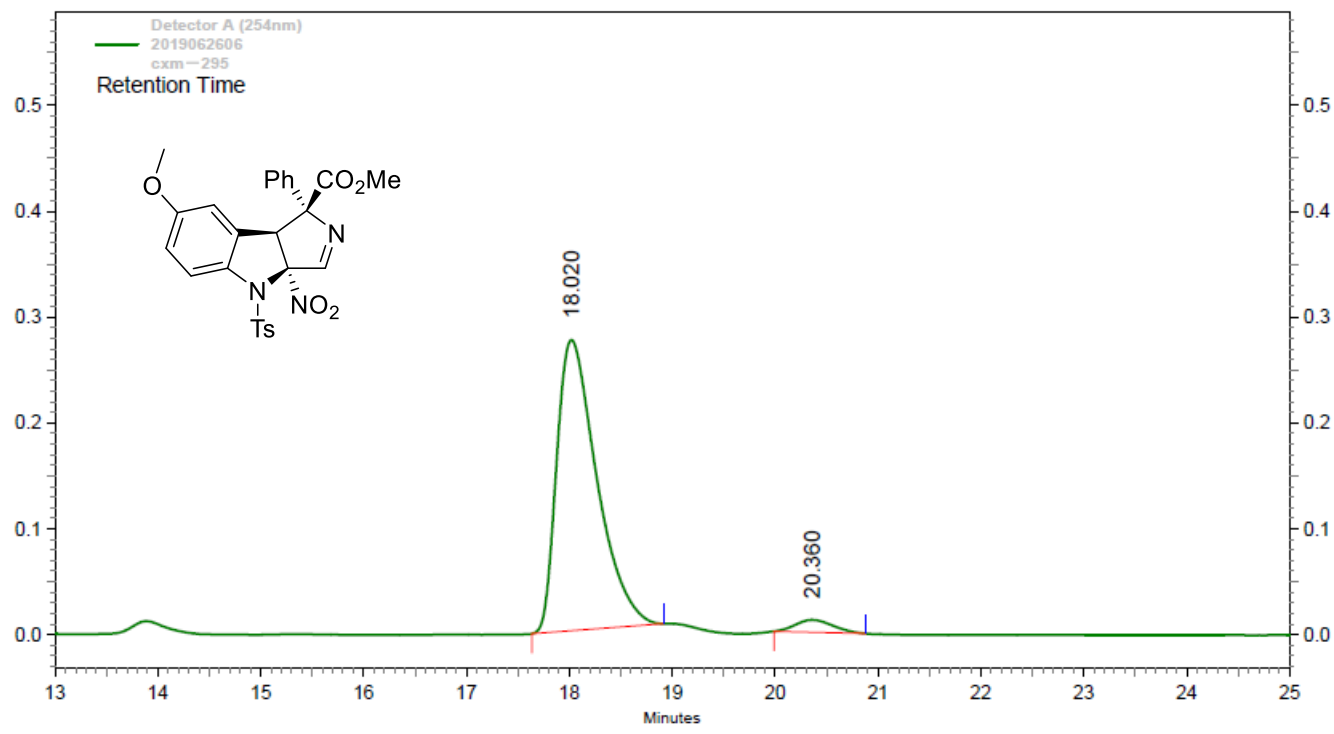

Detector

\begin{tabular}{rrrrrr}
$\begin{array}{r}\text { A (254nm) } \\
\text { Pk \# }\end{array}$ & Retention Time & Height & $\begin{array}{c}\text { Height } \\
\text { Percent }\end{array}$ & Area & Area Percent \\
\hline 1 & 18.020 & 274672 & 95.92 & 7433737 & 96.18 \\
2 & 20.360 & 11683 & 4.08 & 295485 & 3.82
\end{tabular}

\begin{tabular}{|c|c|c|c|c|}
\hline \multirow[t]{2}{*}{ Totals } & & & & \\
\hline & 286355 & 100.00 & 7729222 & 100.00 \\
\hline
\end{tabular}


${ }^{1} \mathrm{H}$ NMR, ${ }^{13} \mathrm{C}$ NMR and HPLC of $3 \mathrm{~g}$

(300 $\mathrm{MHz}, \mathrm{CDCl}_{3},{ }^{1} \mathrm{H}$ NMR)

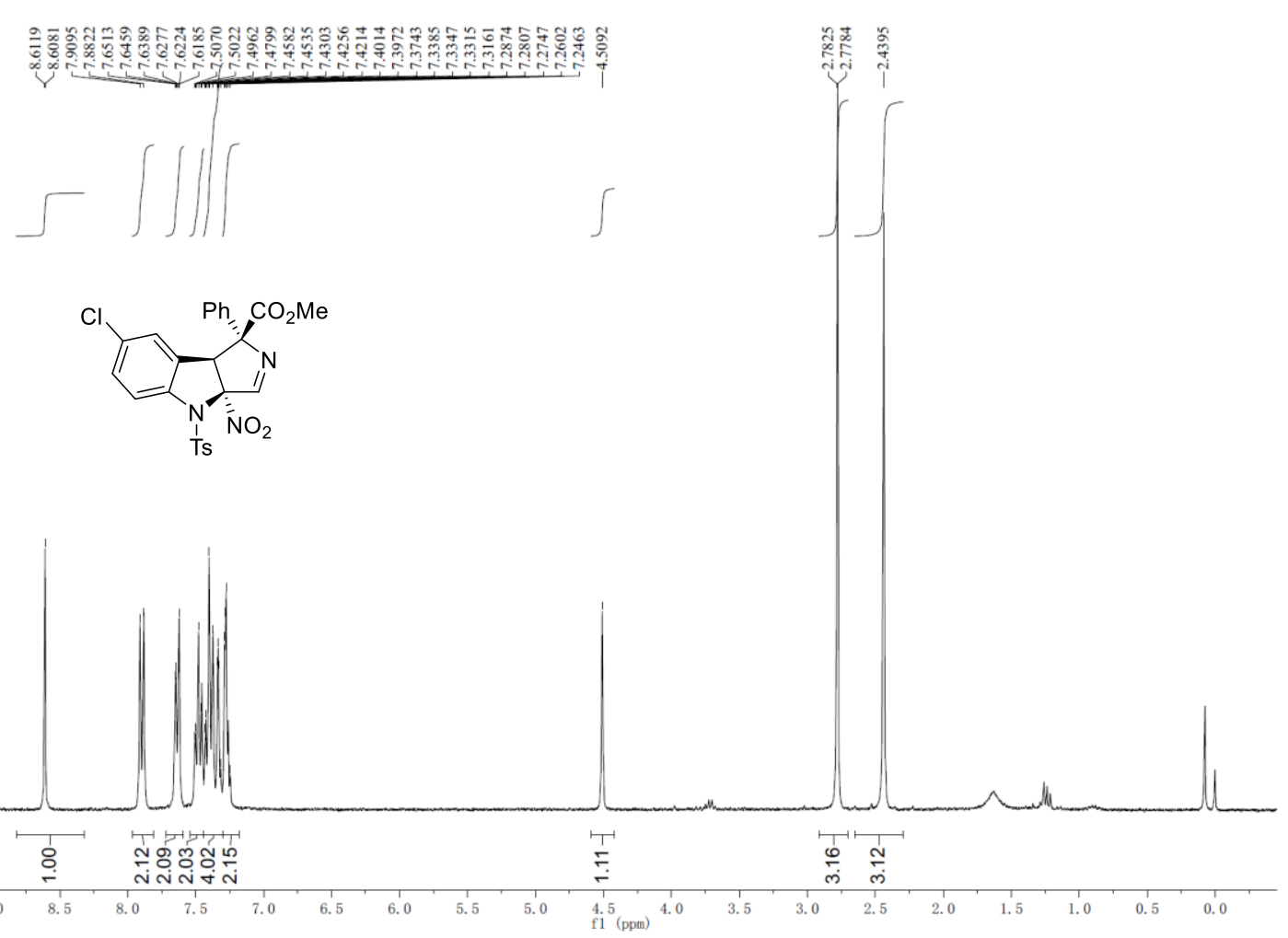

(75 MHz, $\left.\mathrm{CDCl}_{3},{ }^{13} \mathrm{C} \mathrm{NMR}\right)$

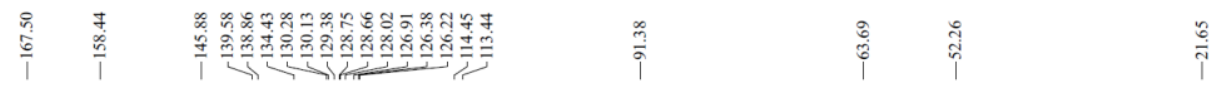

(I)

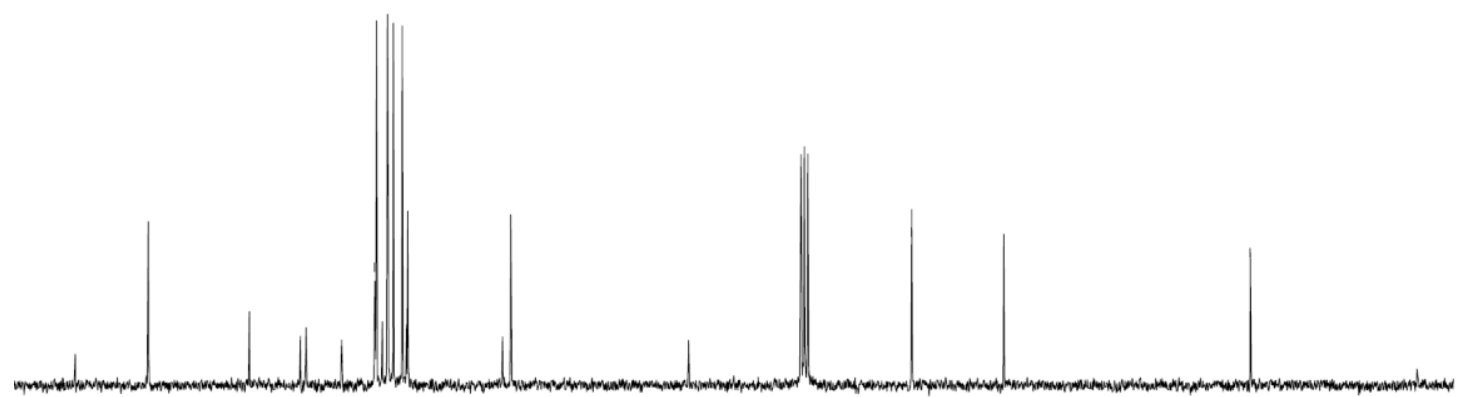

$\begin{array}{ccccccccc}170 & 160 & 150 & 140 & 130 & 120 & 110 & 100 & 90 \\ \mathrm{f} 1(\mathrm{ppm}) & 1\end{array}$ 
$\mathrm{mV}$

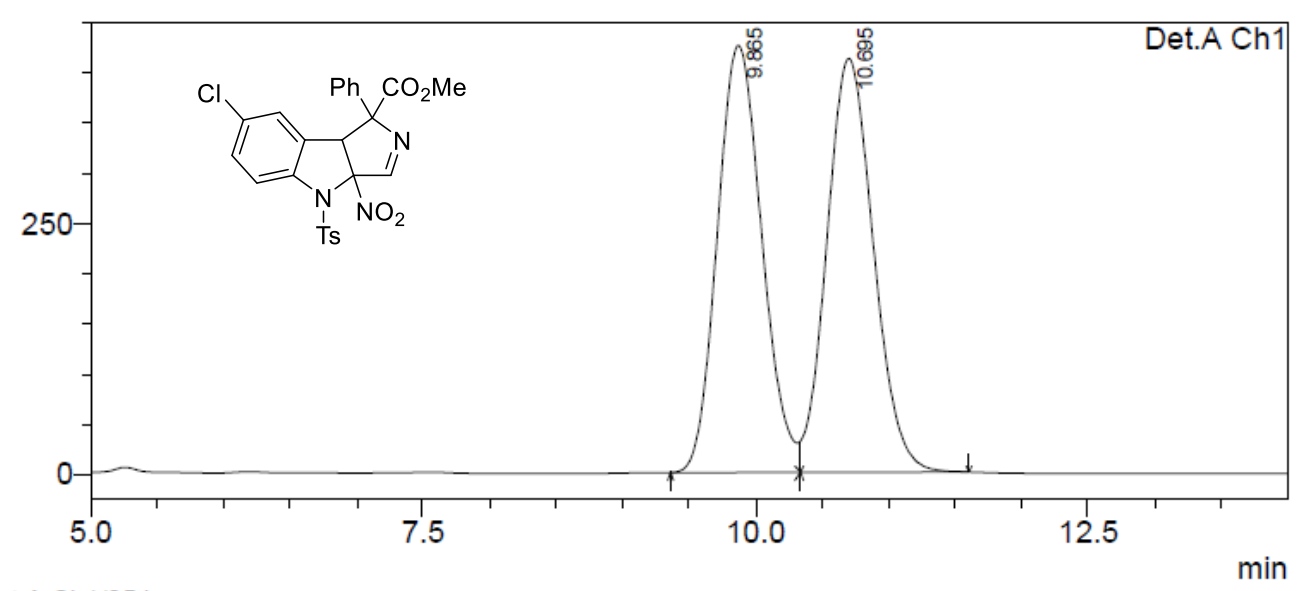

1 Det.A Ch1/254nm

PeakTable

Detector A Ch1 $254 \mathrm{~nm}$

\begin{tabular}{|c|c|c|c|c|}
\hline Peak\# & Ret. Time & Area & Height & Area \% \\
\hline 1 & 9.865 & 9961601 & 427002 & 49.630 \\
\hline 2 & 10.695 & 10110199 & 413599 & 50.370 \\
\hline Total & & 20071800 & & 100.000 \\
\hline
\end{tabular}

$\mathrm{mV}$

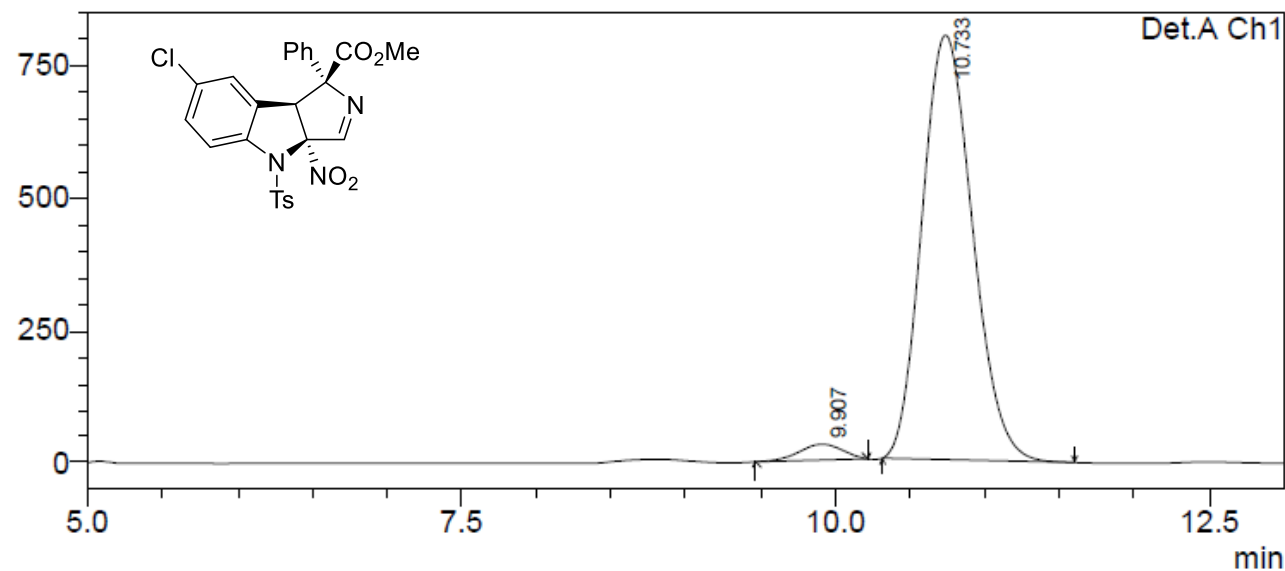

1 Det.A Ch1/254nm

PeakTable

Detector A Ch1 $254 \mathrm{~nm}$
\begin{tabular}{|c|c|c|c|c|}
\hline Peak\# & Ret. Time & Area & Height & Area $\%$ \\
\hline 1 & 9.907 & 595423 & 29706 & 3.069 \\
\hline 2 & 10.733 & 18807463 & 798877 & 96.931 \\
\hline Total & & 19402886 & & 100.000 \\
\hline
\end{tabular}


${ }^{1} \mathrm{H}$ NMR, ${ }^{13} \mathrm{C}$ NMR and HPLC of $3 \mathrm{~h}$

(300 $\mathrm{MHz}, \mathrm{CDCl}_{3},{ }^{1} \mathrm{H}$ NMR)
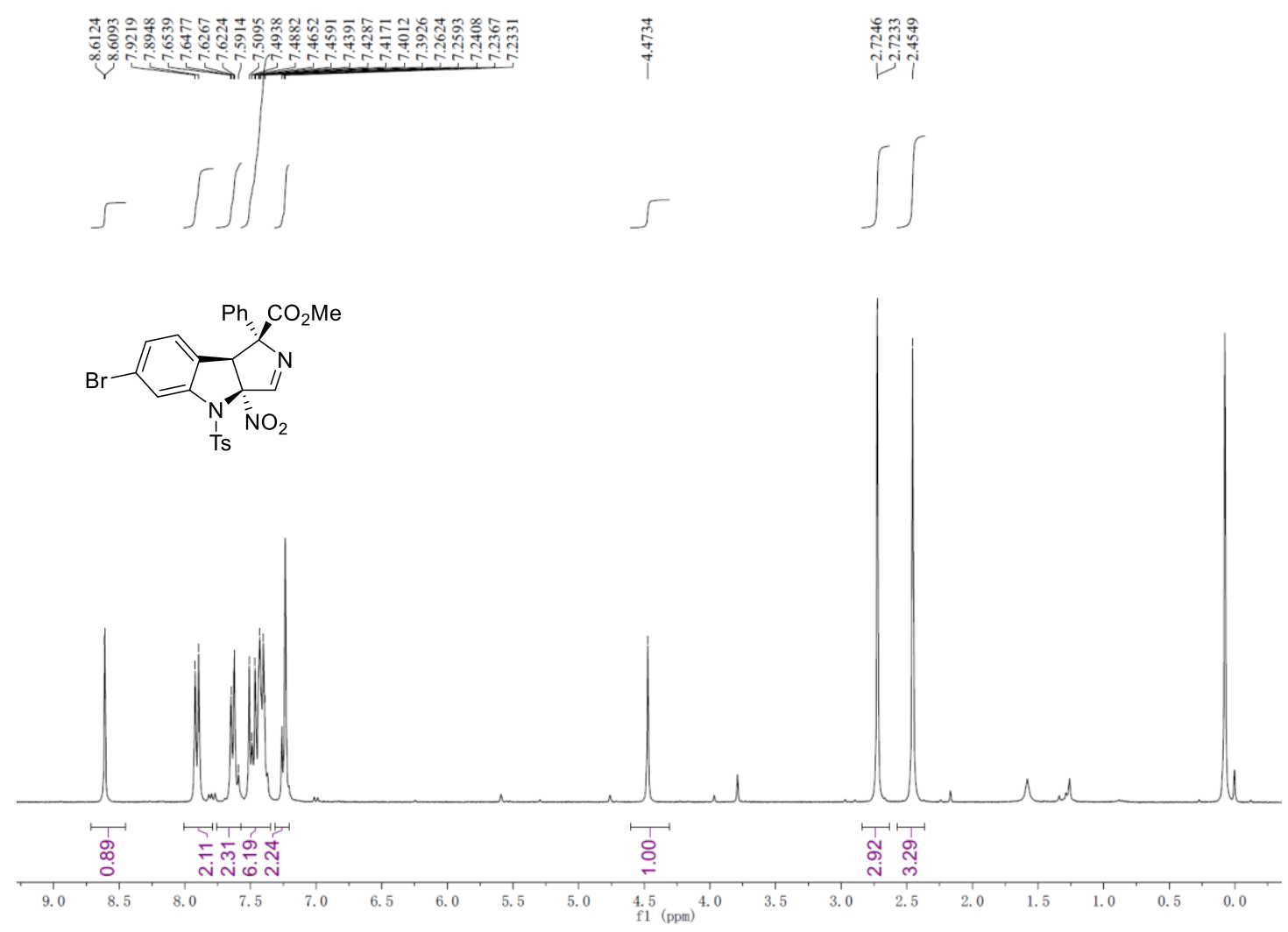

(75 MHz, $\left.\mathrm{CDCl}_{3},{ }^{13} \mathrm{C} \mathrm{NMR}\right)$
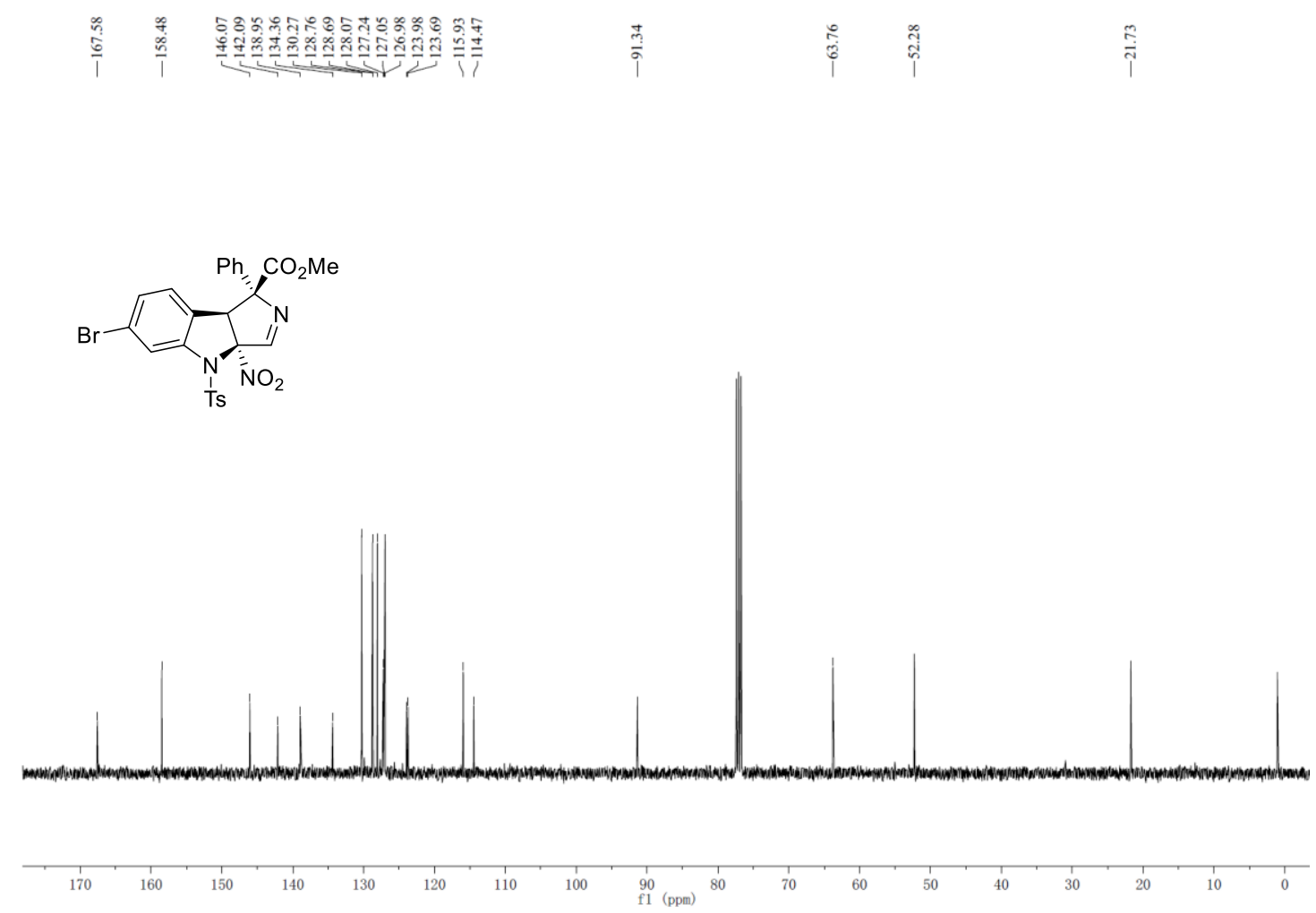


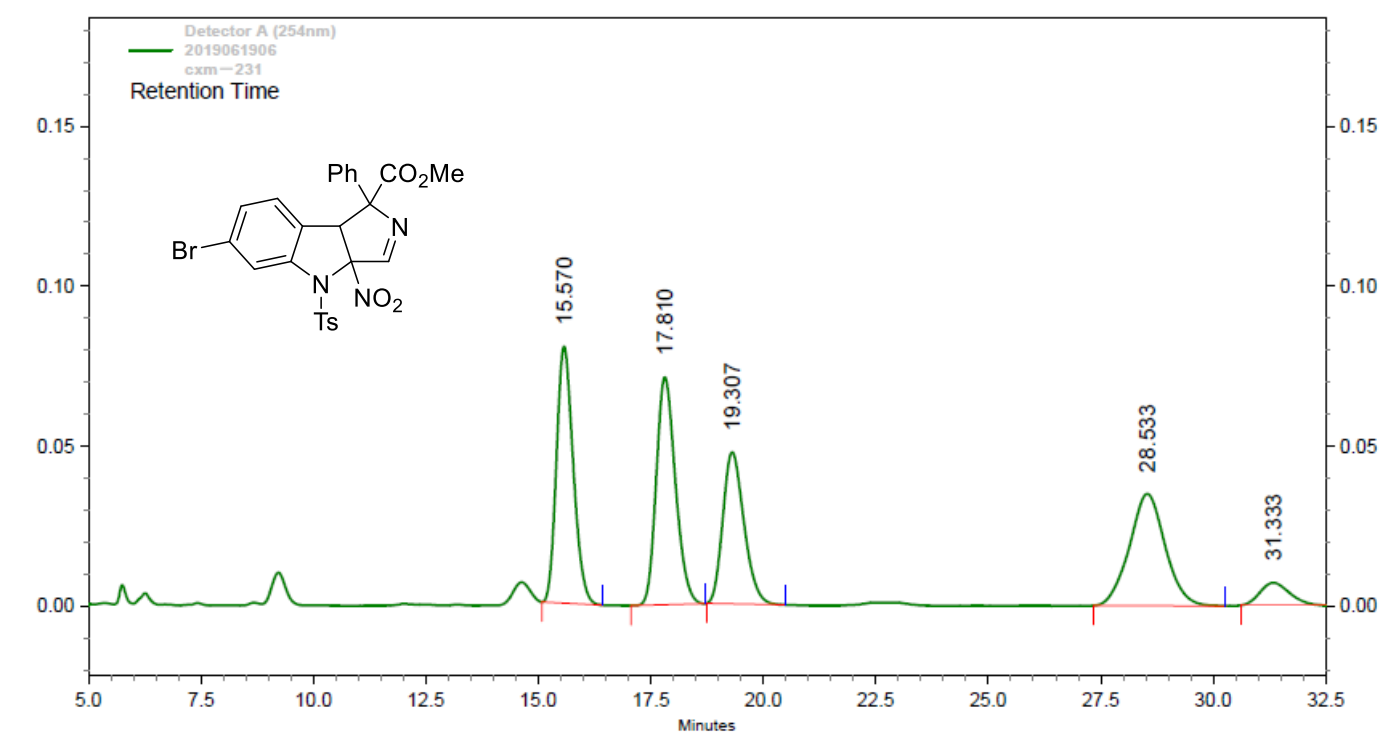

Detector

\begin{tabular}{|c|c|c|c|c|c|}
\hline $\begin{array}{r}A(254 \mathrm{~nm}) \\
\mathrm{Pk} \#\end{array}$ & Retention Time & Height & $\begin{array}{l}\text { Height } \\
\text { Percent }\end{array}$ & Area & Area Percent \\
\hline 1 & 15.570 & 80234 & 33.33 & 2079810 & 26.01 \\
\hline 2 & 17.810 & 71113 & 29.54 & 2107712 & 26.36 \\
\hline 3 & 19.307 & 47384 & 19.69 & 1553646 & 19.43 \\
\hline 4 & 28.533 & 34970 & 14.53 & 1931639 & 24.16 \\
\hline 5 & 31.333 & 7008 & 2.91 & 321866 & 4.03 \\
\hline
\end{tabular}

\begin{tabular}{|c|c|c|c|c|}
\hline \multirow[t]{2}{*}{ Totals } & & & & \\
\hline & 240709 & 100.00 & 7994673 & 100.00 \\
\hline
\end{tabular}

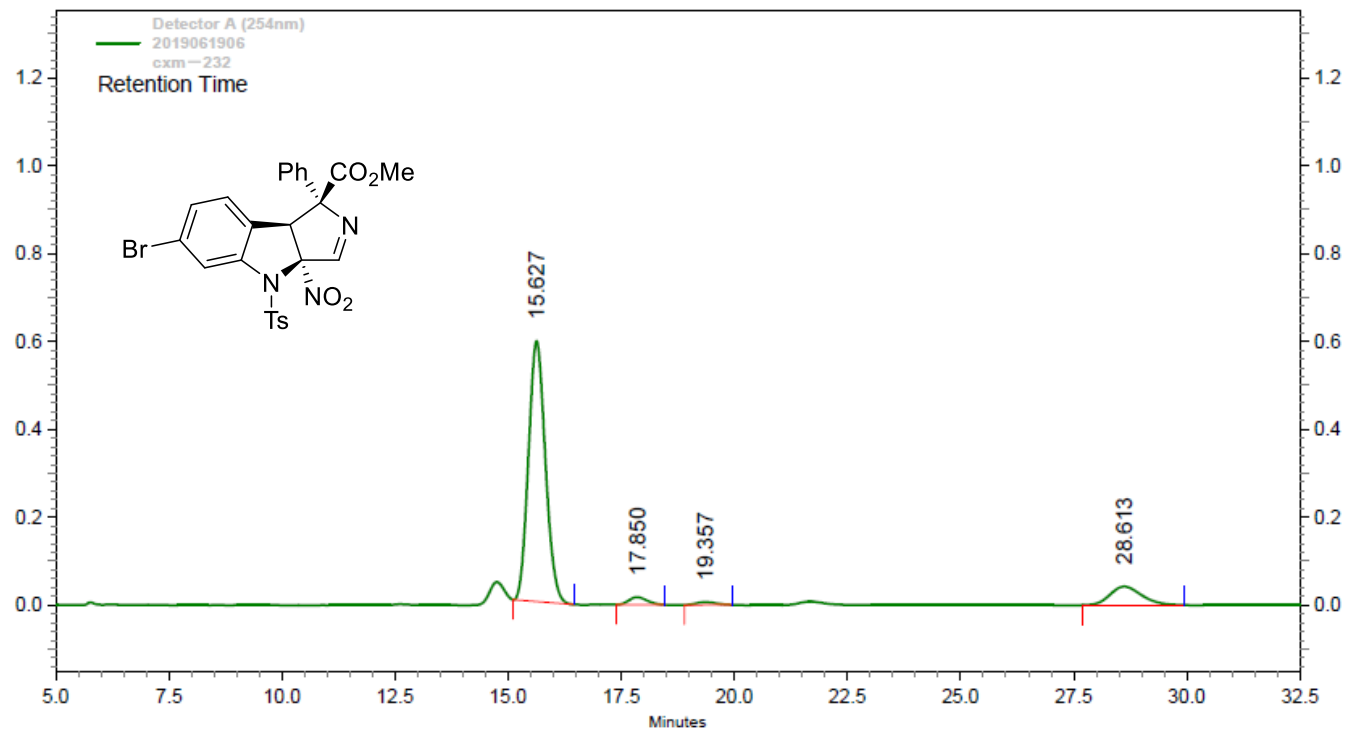

Detector

\begin{tabular}{|c|c|c|c|c|c|}
\hline $\begin{array}{r}A(254 \mathrm{~nm}) \\
\mathrm{Pk} \#\end{array}$ & Retention Time & Height & $\begin{array}{l}\text { Height } \\
\text { Percent }\end{array}$ & Area & Area Percent \\
\hline 1 & 15.627 & 592858 & 90.03 & 15453463 & 85.06 \\
\hline 2 & 17.850 & 17185 & 2.61 & 480997 & 2.65 \\
\hline 3 & 19.357 & 6426 & 0.98 & 193155 & 1.06 \\
\hline 4 & 28.613 & 42058 & 6.39 & 2039416 & 11.23 \\
\hline
\end{tabular}

\begin{tabular}{|c|c|c|c|c|}
\hline \multirow[t]{2}{*}{ Totals } & & & & \\
\hline & 658527 & 100.00 & 18167031 & 100.00 \\
\hline
\end{tabular}


${ }^{1} \mathrm{H}$ NMR, ${ }^{13} \mathrm{C}$ NMR and HPLC of $3 i$

(300 MHz, $\mathrm{CDCl}_{3},{ }^{1} \mathrm{H}$ NMR)
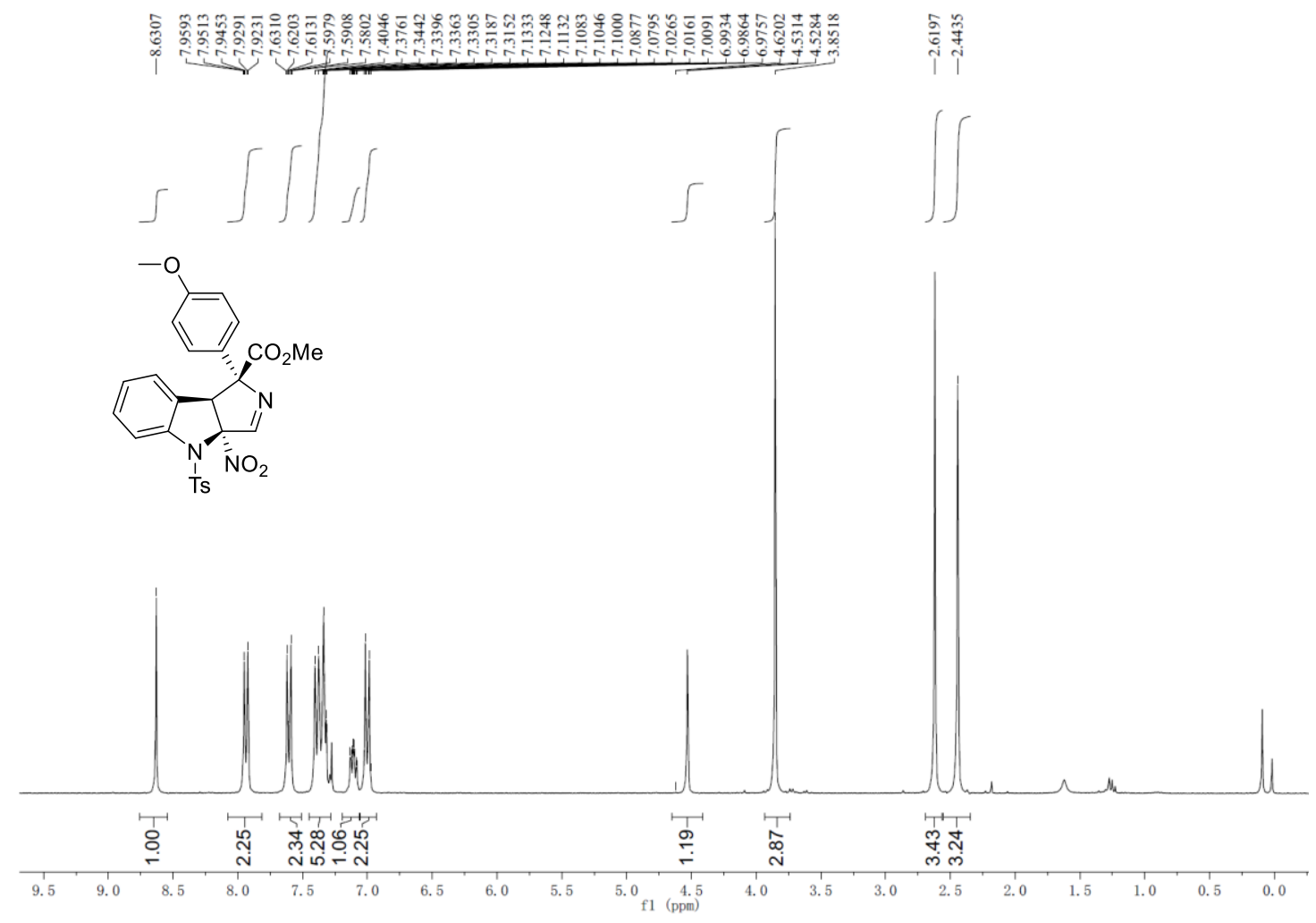

(75 $\left.\mathrm{MHz}, \mathrm{CDCl}_{3},{ }^{13} \mathrm{C} \mathrm{NMR}\right)$
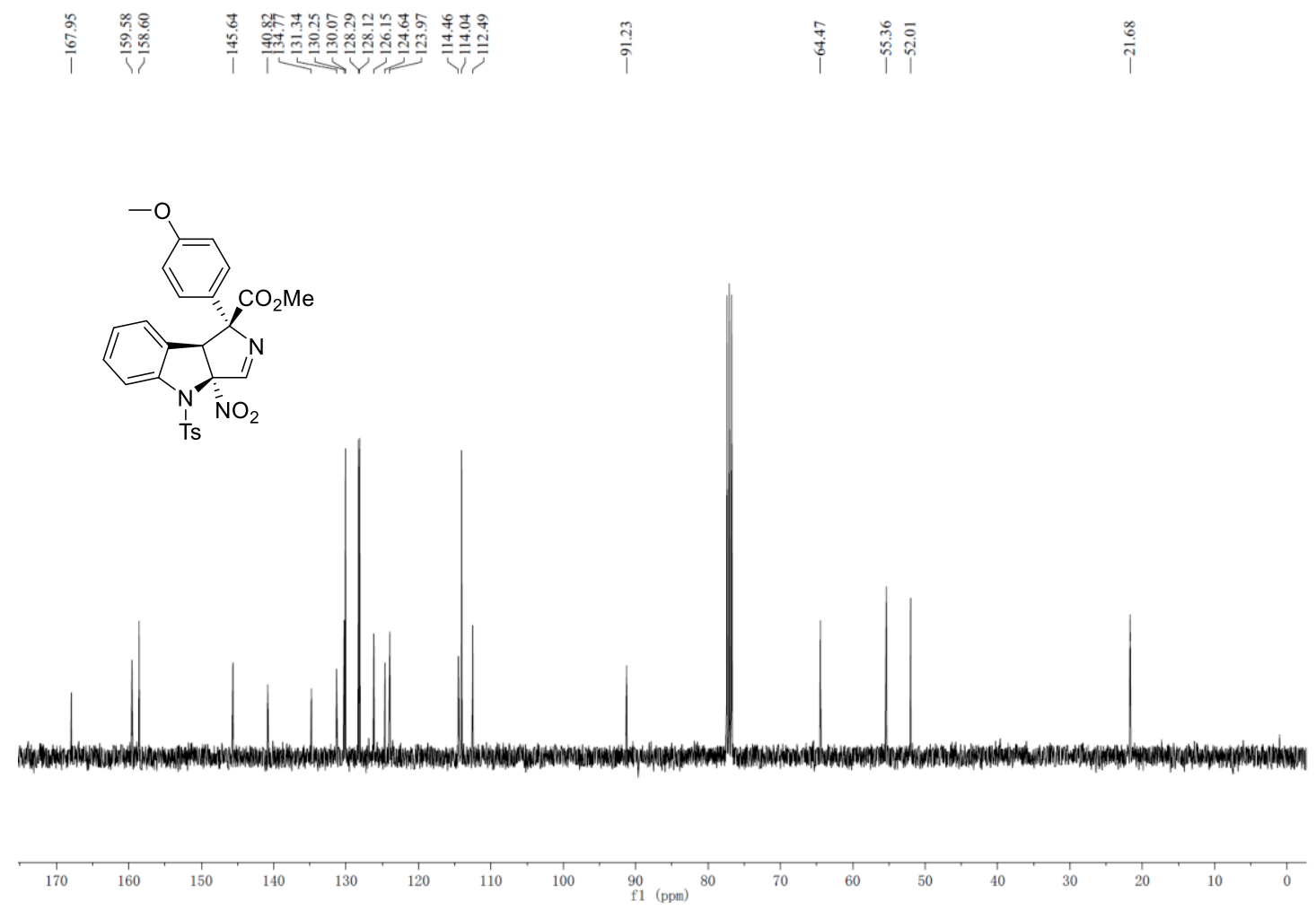

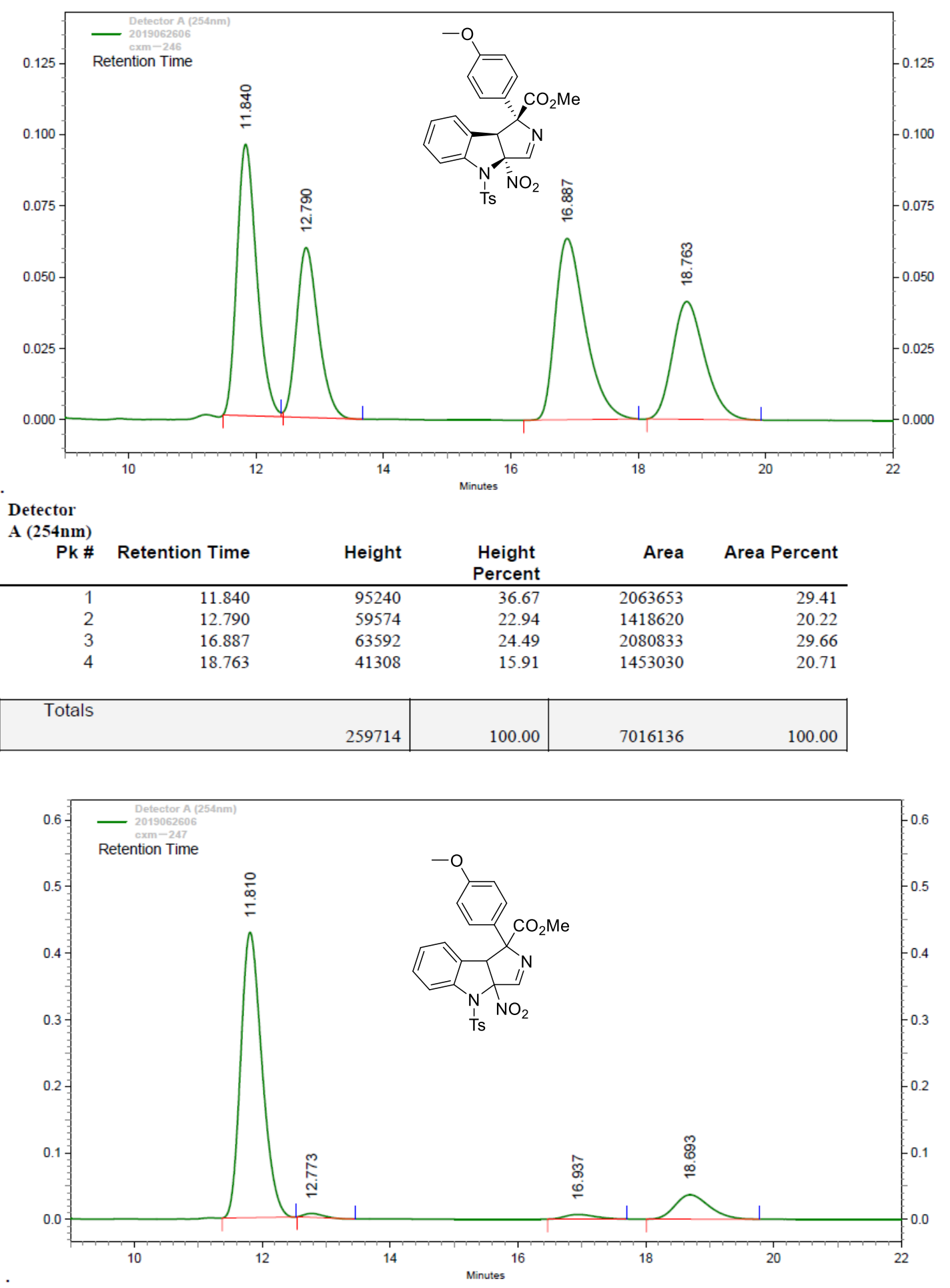

Detector

\begin{tabular}{rrrrrr}
$\begin{array}{r}\text { A (254nm) } \\
\text { Pk \# }\end{array}$ & Retention Time & Height & $\begin{array}{r}\text { Height } \\
\text { Percent }\end{array}$ & Area & Area Percent \\
\hline 1 & 11.810 & 428695 & 89.66 & 9914089 & 85.36 \\
2 & 12.773 & 5876 & 1.23 & 113150 & 0.97 \\
3 & 16.937 & 6918 & 1.45 & 227443 & 1.96 \\
4 & 18.693 & 36638 & 7.66 & 1360102 & 11.71 \\
\hline \multicolumn{2}{|c|}{} & 478127 & 100.00 & 11614784 & 100.00 \\
\hline
\end{tabular}


${ }^{1} \mathrm{H}$ NMR, ${ }^{13} \mathrm{C}$ NMR and HPLC of $3 \mathrm{j}$

(300 $\mathrm{MHz}, \mathrm{CDCl}_{3},{ }^{1} \mathrm{H}$ NMR)

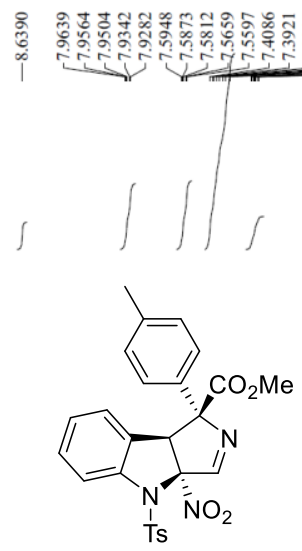

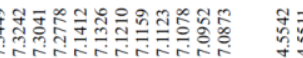

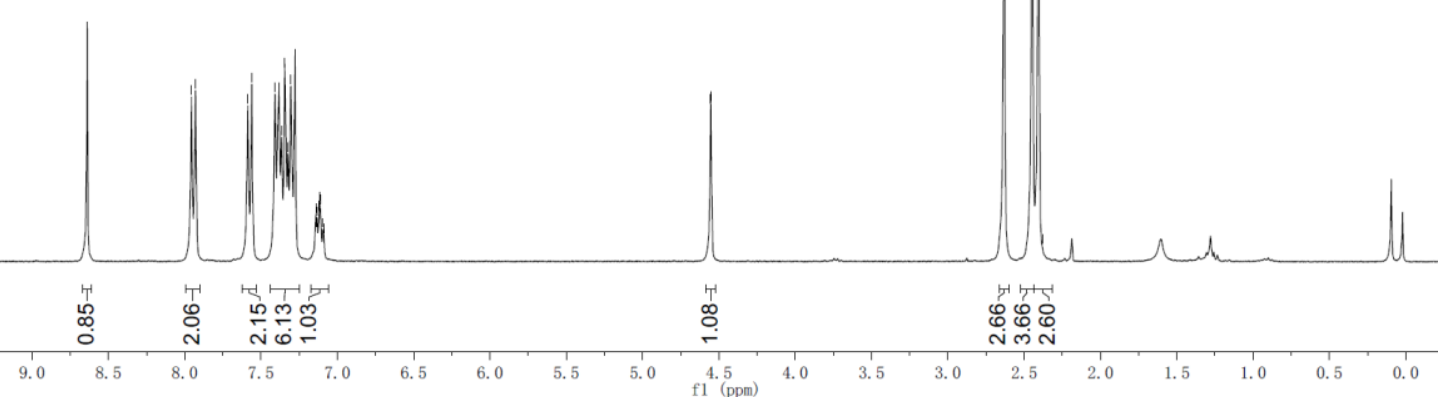

$\left(75 \mathrm{~Hz}, \mathrm{CDCl}_{3},{ }^{13} \mathrm{C} \mathrm{NMR}\right)$

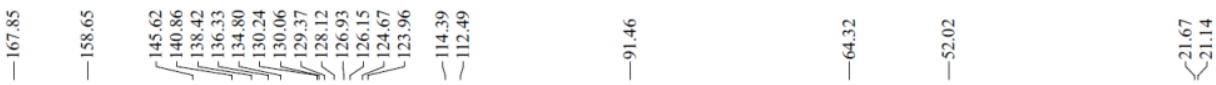<smiles>Cc1ccc(C2(C(N)([O-])O)C=NC3C(c4ccccc4N3C)N2C)cc1</smiles>

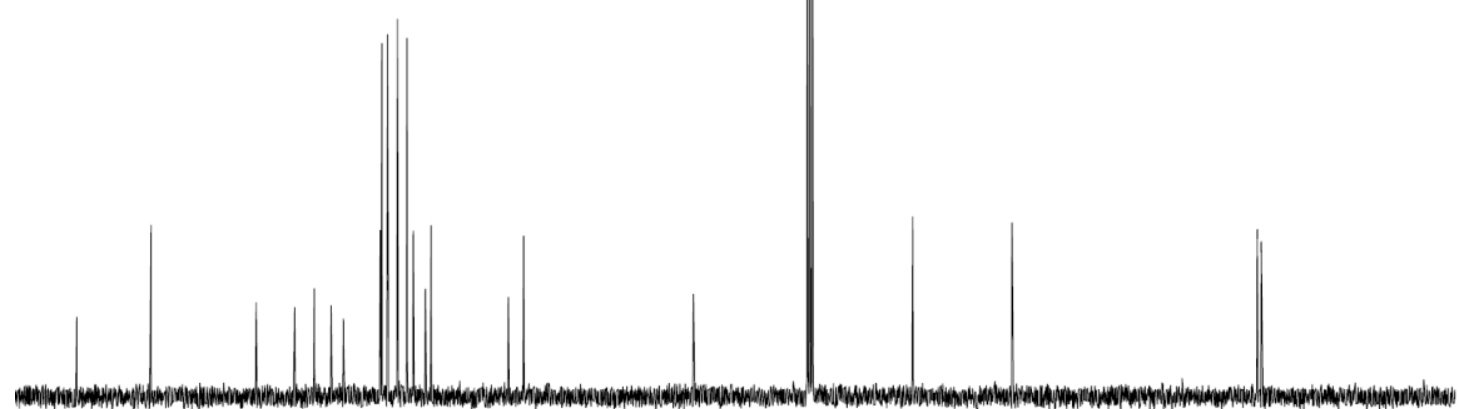

$\begin{array}{lllllllll}170 & 160 & 150 & 140 & 130 & 120 & 110 & 100 & 90 \\ \mathrm{f} 1 & 9(\mathrm{ppm})\end{array}$ 


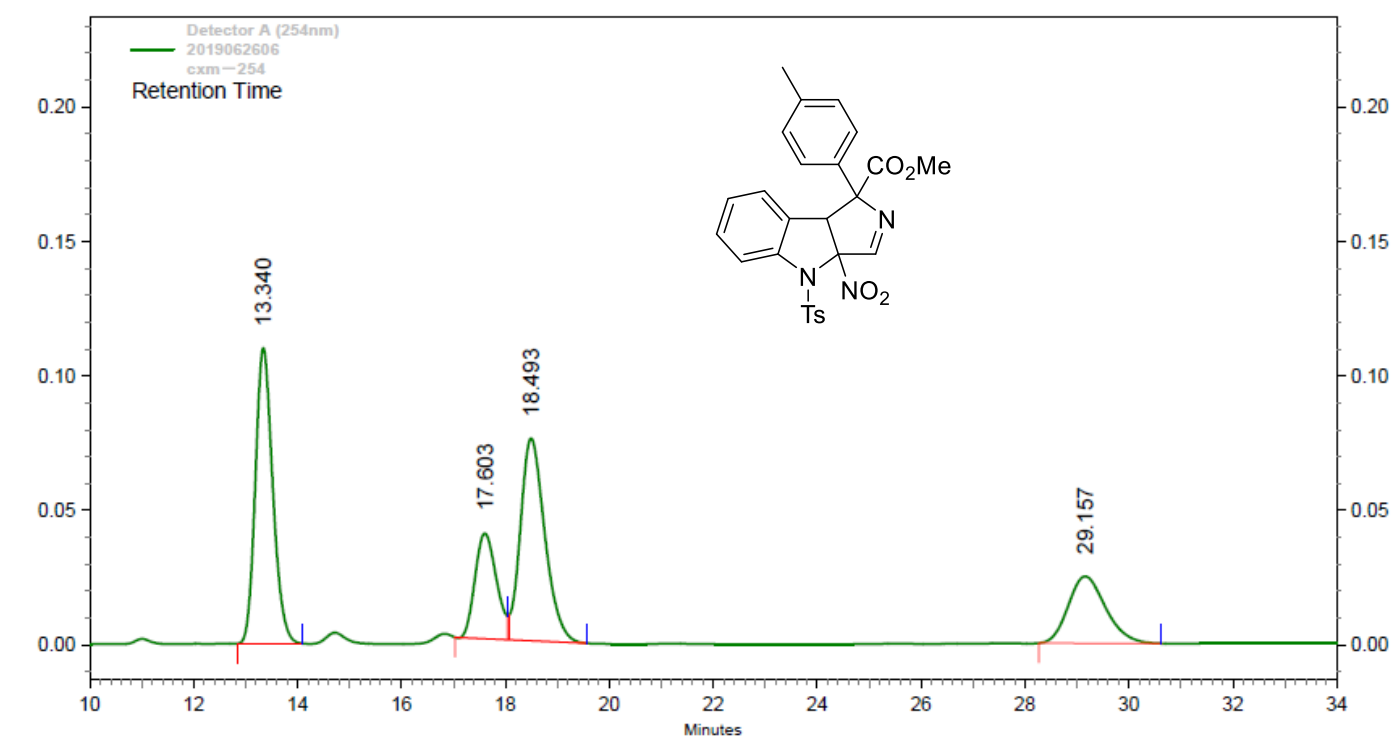

Detector

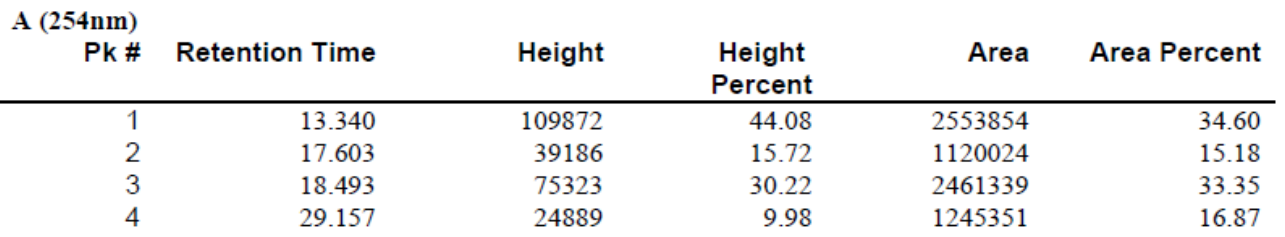

\begin{tabular}{|c|c|c|c|c|}
\hline Totals & & & & \\
\hline & 249270 & 100.00 & 7380568 & 100.00 \\
\hline
\end{tabular}

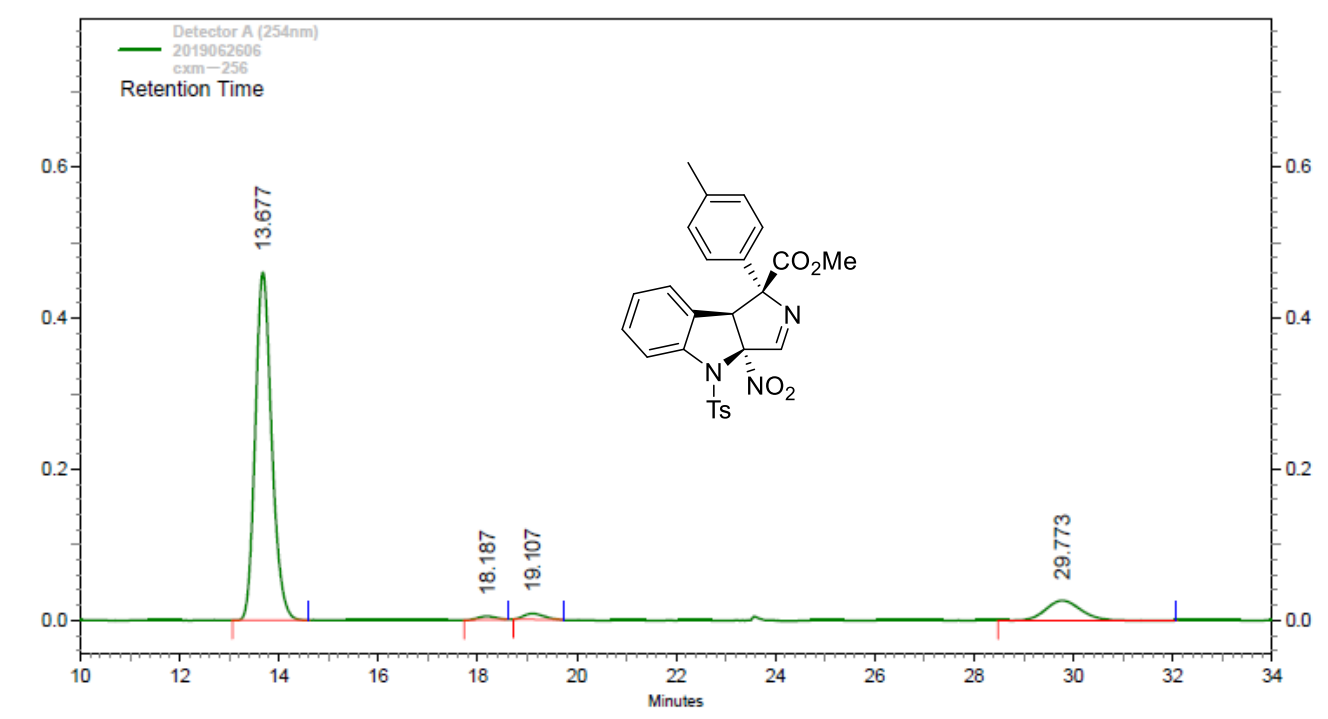

Detector

\begin{tabular}{rrrrrr}
$\begin{array}{r}\text { A (254nm) } \\
\text { Pk \# }\end{array}$ & Retention Time & Height & $\begin{array}{c}\text { Height } \\
\text { Percent }\end{array}$ & Area & Area Percent \\
\hline 1 & 13.677 & 460515 & 92.19 & 10592228 & 86.50 \\
2 & 18.187 & 4696 & 0.94 & 121348 & 0.99 \\
3 & 19.107 & 7838 & 1.57 & 222915 & 1.82 \\
4 & 29.773 & 26497 & 5.30 & 1309353 & 10.69
\end{tabular}

\begin{tabular}{|r|rrrrr|}
\hline Totals & & & & & \\
& 499546 & 100.00 & 12245844 & 100.00 \\
\hline
\end{tabular}


${ }^{1} \mathrm{H}$ NMR, ${ }^{13} \mathrm{C}$ NMR and HPLC of $3 \mathrm{k}$

(300 $\mathrm{MHz}, \mathrm{CDCl}_{3},{ }^{1} \mathrm{H} \mathrm{NMR}$ )
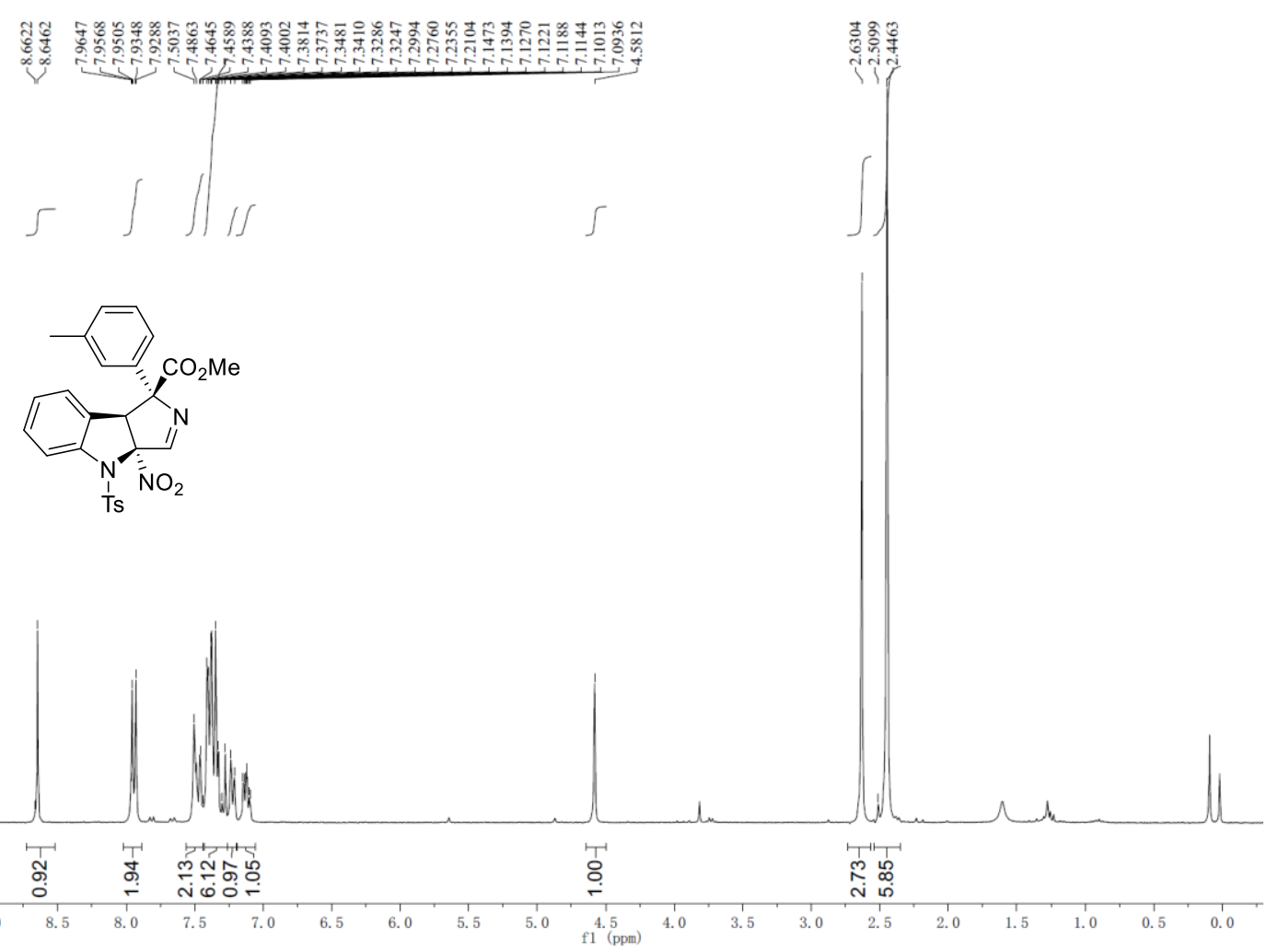

$\left(75 \mathrm{MHz}, \mathrm{CDCl}_{3},{ }^{13} \mathrm{C} \mathrm{NMR}\right)$
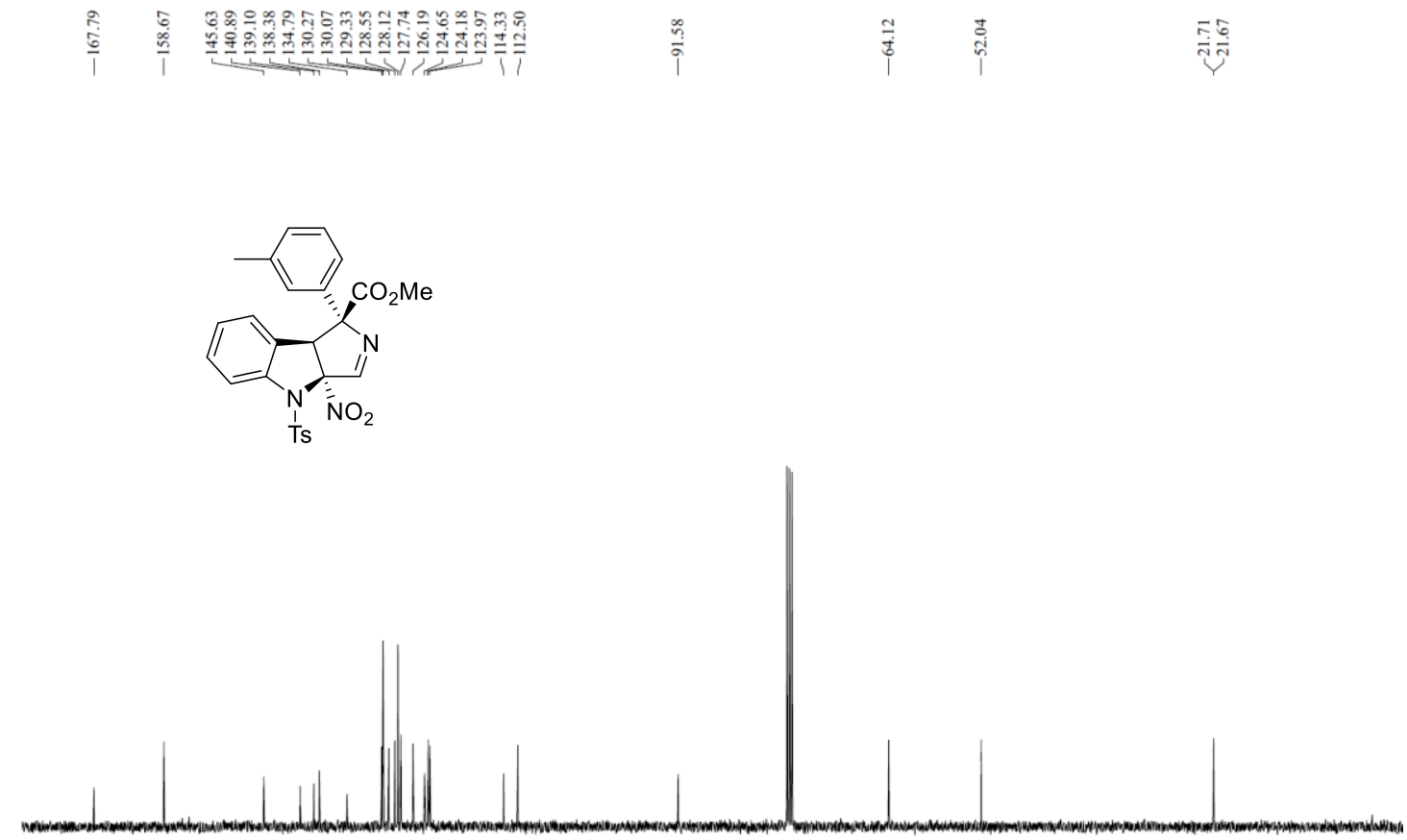

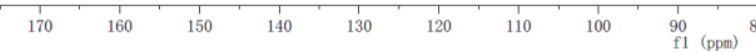




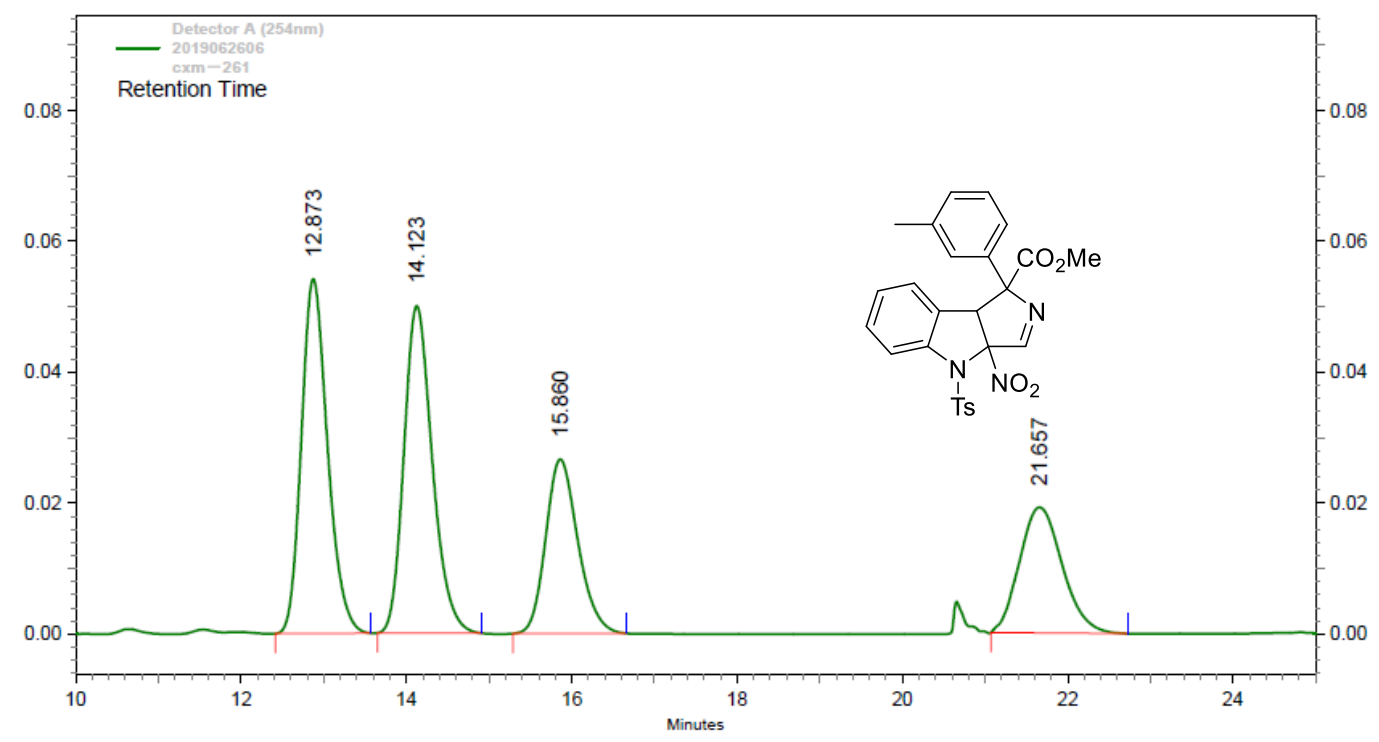

Detector

\begin{tabular}{|c|c|c|c|c|c|}
\hline $\begin{array}{r}A(254 n m) \\
P k \#\end{array}$ & Retention Time & Height & $\begin{array}{l}\text { Height } \\
\text { Percent }\end{array}$ & Area & Area Percent \\
\hline 1 & 12.873 & 54145 & 36.12 & 1189098 & 31.36 \\
\hline 2 & 14.123 & 49961 & 33.33 & 1192305 & 31.44 \\
\hline 3 & 15.860 & 26611 & 17.75 & 720209 & 18.99 \\
\hline 4 & 21.657 & 19179 & 12.79 & 690192 & 18.20 \\
\hline
\end{tabular}

\begin{tabular}{|l|r|r|rr|}
\hline Totals & 149896 & 100.00 & 3791804 & 100.00 \\
\hline
\end{tabular}

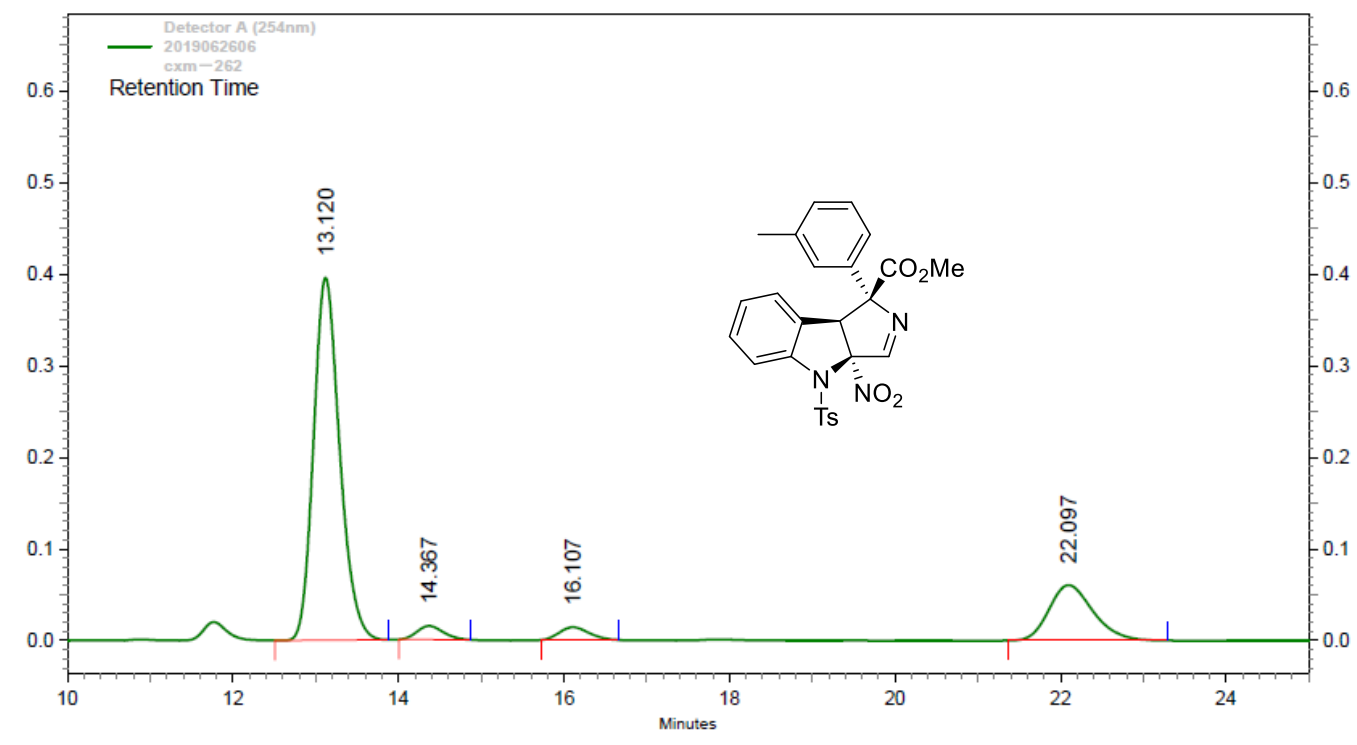

Detector

\begin{tabular}{|c|c|c|c|c|c|}
\hline $\begin{array}{r}A(254 \mathrm{~nm}) \\
\mathrm{Pk} \#\end{array}$ & Retention Time & Height & $\begin{array}{l}\text { Height } \\
\text { Percent }\end{array}$ & Area & Area Percent \\
\hline 1 & 13.120 & 395623 & 81.58 & 8670285 & 74.75 \\
\hline 2 & 14.367 & 14881 & 3.07 & 330676 & 2.85 \\
\hline 3 & 16.107 & 13924 & 2.87 & 350944 & 3.03 \\
\hline 4 & 22.097 & 60534 & 12.48 & 2247303 & 19.37 \\
\hline
\end{tabular}

\begin{tabular}{|c|c|c|c|c|}
\hline Totals & & & & \\
\hline & 484962 & 100.00 & 11599208 & 100.00 \\
\hline
\end{tabular}


${ }^{1} \mathrm{H}$ NMR, ${ }^{13} \mathrm{C}$ NMR, ${ }^{19} \mathrm{~F}$ NMR and HPLC of 31

(300 $\mathrm{MHz}, \mathrm{CDCl}_{3},{ }^{1} \mathrm{H}$ NMR)

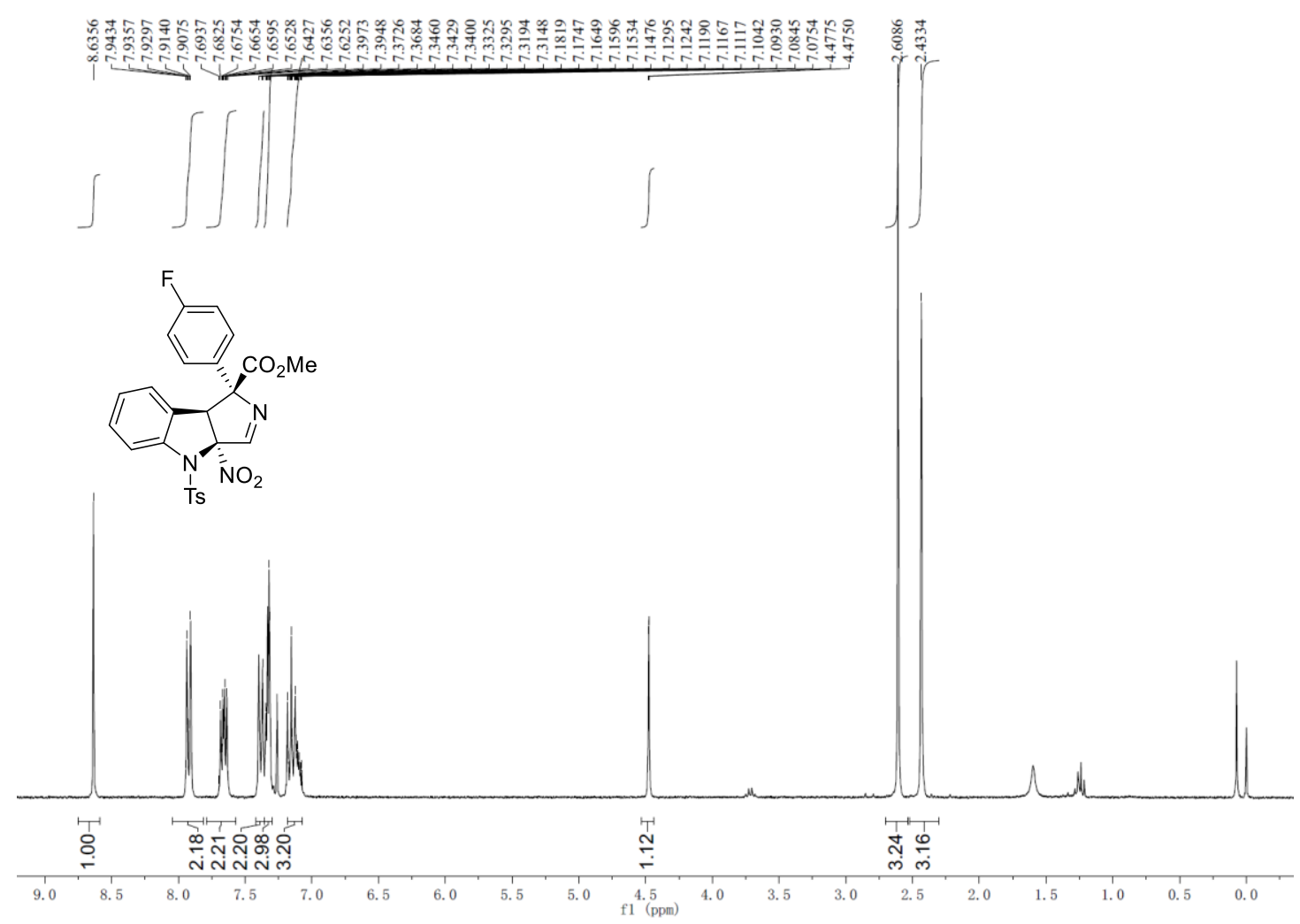

(75 $\mathrm{MHz}, \mathrm{CDCl}_{3},{ }^{13} \mathrm{C}$ NMR)
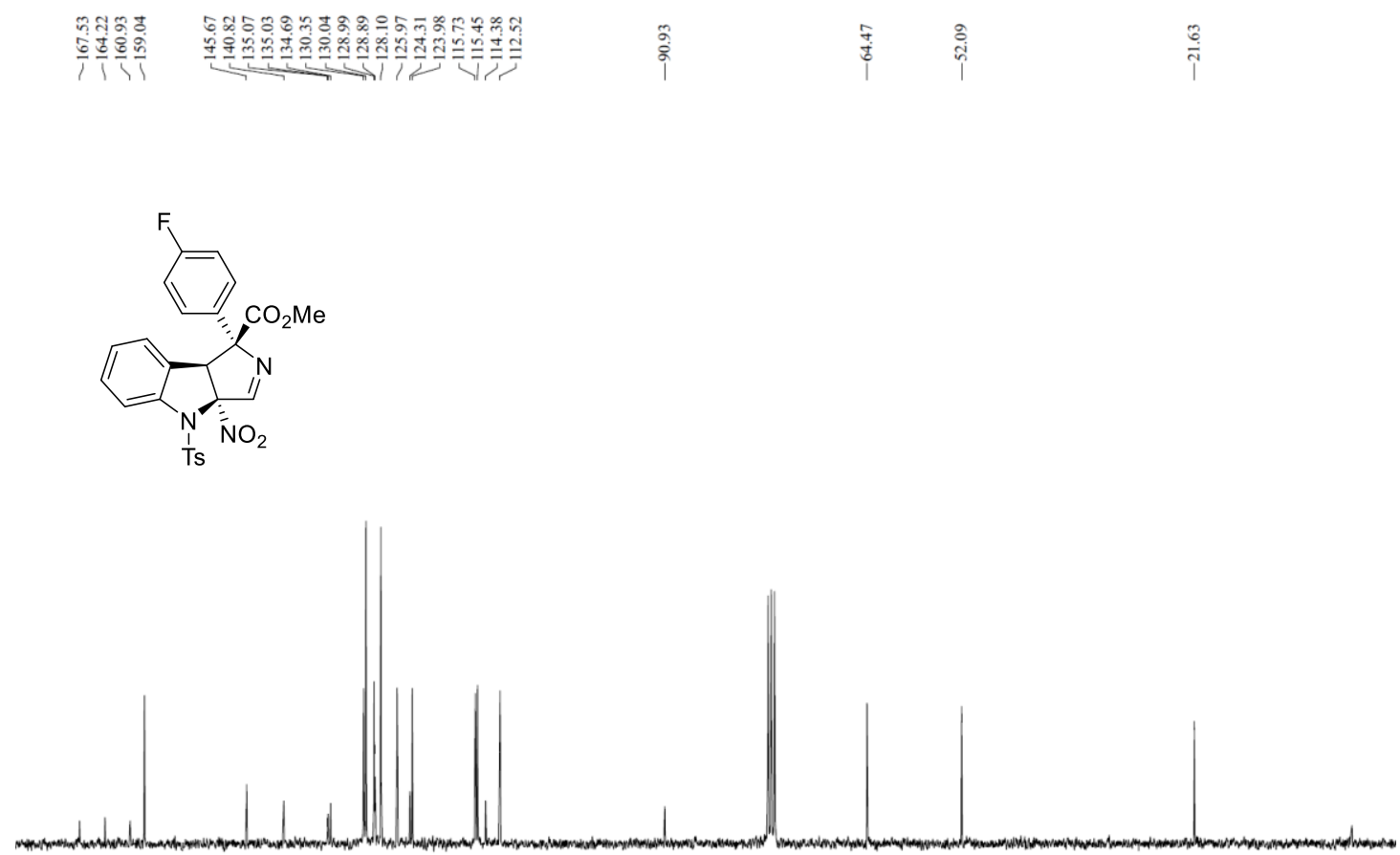

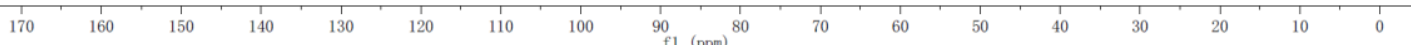


(376 MHz, $\mathrm{CDCl}_{3},{ }^{19} \mathrm{~F}$ NMR)

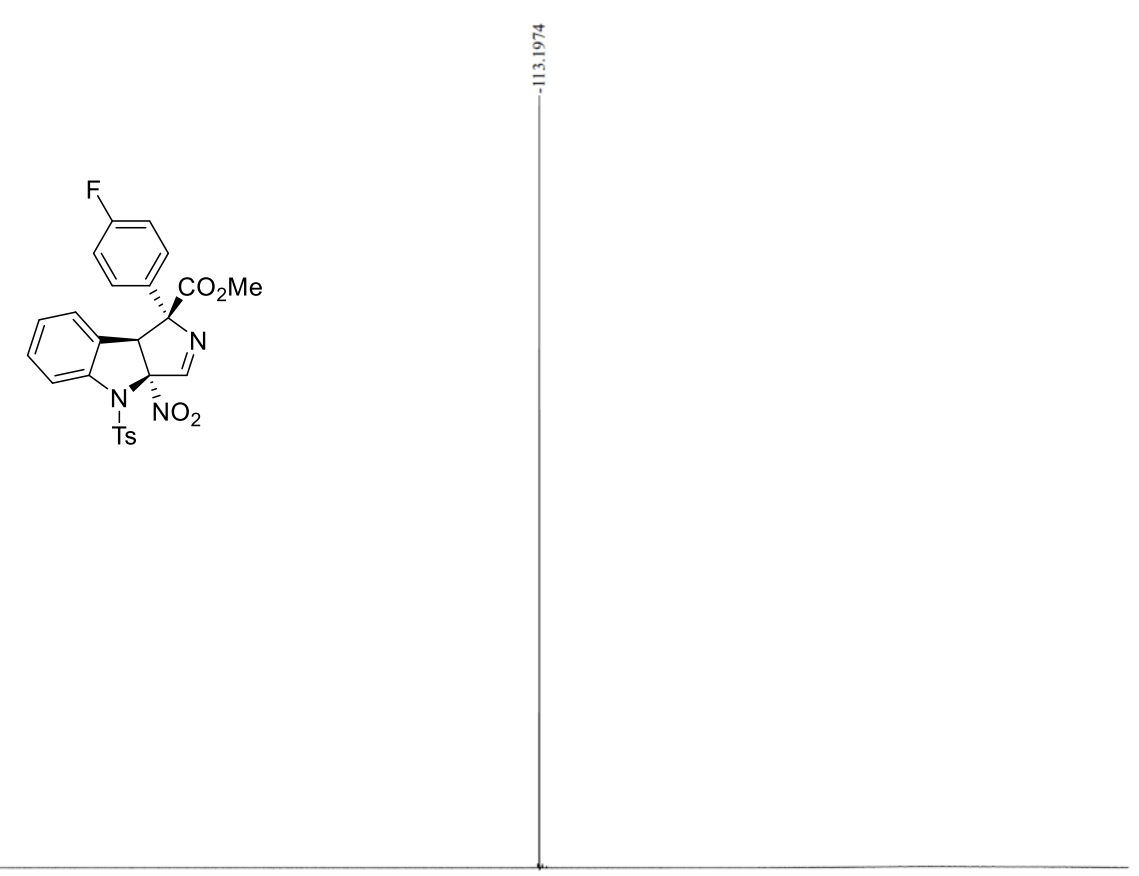

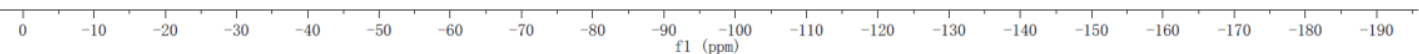




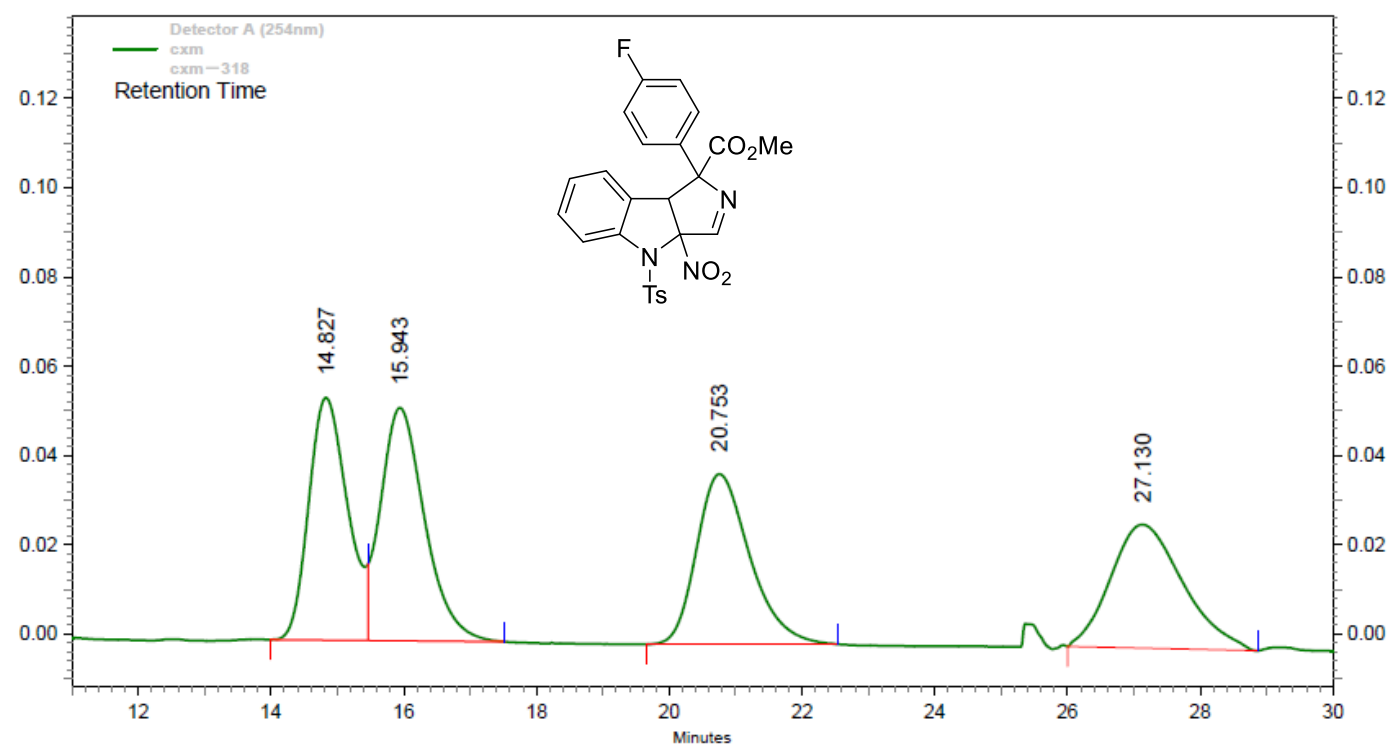

Detector

\begin{tabular}{|c|c|c|c|c|c|}
\hline $\begin{array}{r}A(254 \mathrm{~nm}) \\
\mathrm{Pk} \#\end{array}$ & Retention Time & Height & $\begin{array}{l}\text { Height } \\
\text { Percent }\end{array}$ & Area & Area Percent \\
\hline 1 & 14.827 & 54276 & 31.52 & 2223044 & 25.14 \\
\hline 2 & 15.943 & 52158 & 30.29 & 2408850 & 27.24 \\
\hline 3 & 20.753 & 38086 & 22.12 & 2121010 & 23.99 \\
\hline 4 & 27.130 & 27660 & 16.06 & 2089740 & 23.63 \\
\hline
\end{tabular}

\begin{tabular}{|c|c|c|c|c|}
\hline Totals & & & & \\
\hline & 172180 & 100.00 & 8842644 & 100.00 \\
\hline
\end{tabular}

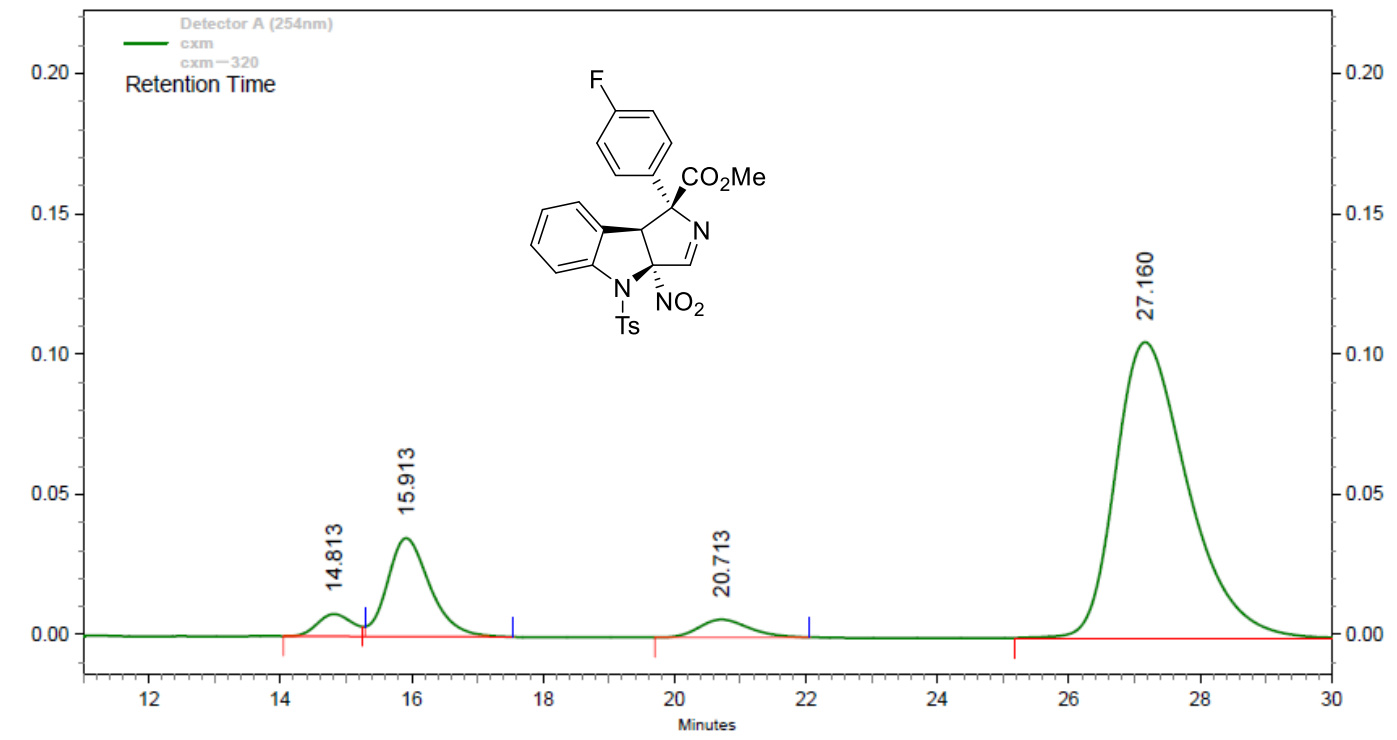

Detector

A (254nm)

Pk \# Retention Time

\begin{tabular}{ll}
\hline 1 & 14.813 \\
2 & 15.913 \\
3 & 20.713 \\
4 & 27.160
\end{tabular}

14.813

15.913

20.713

27.160

\begin{tabular}{r} 
Height \\
\hline 7915 \\
35066 \\
6359 \\
105399
\end{tabular}

Height Percent

Area Area Percent

7915

5.12

22.66

4.11

1588216

336967

8050134

(1) 
${ }^{1} \mathrm{H}$ NMR, ${ }^{13} \mathrm{C}$ NMR and HPLC of $3 \mathrm{~m}$

(300 $\mathrm{MHz}, \mathrm{CDCl}_{3},{ }^{1} \mathrm{H}$ NMR)

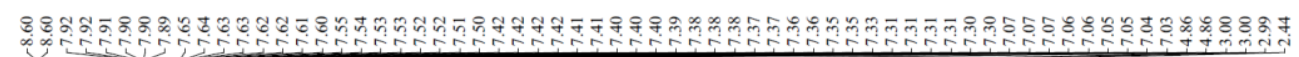
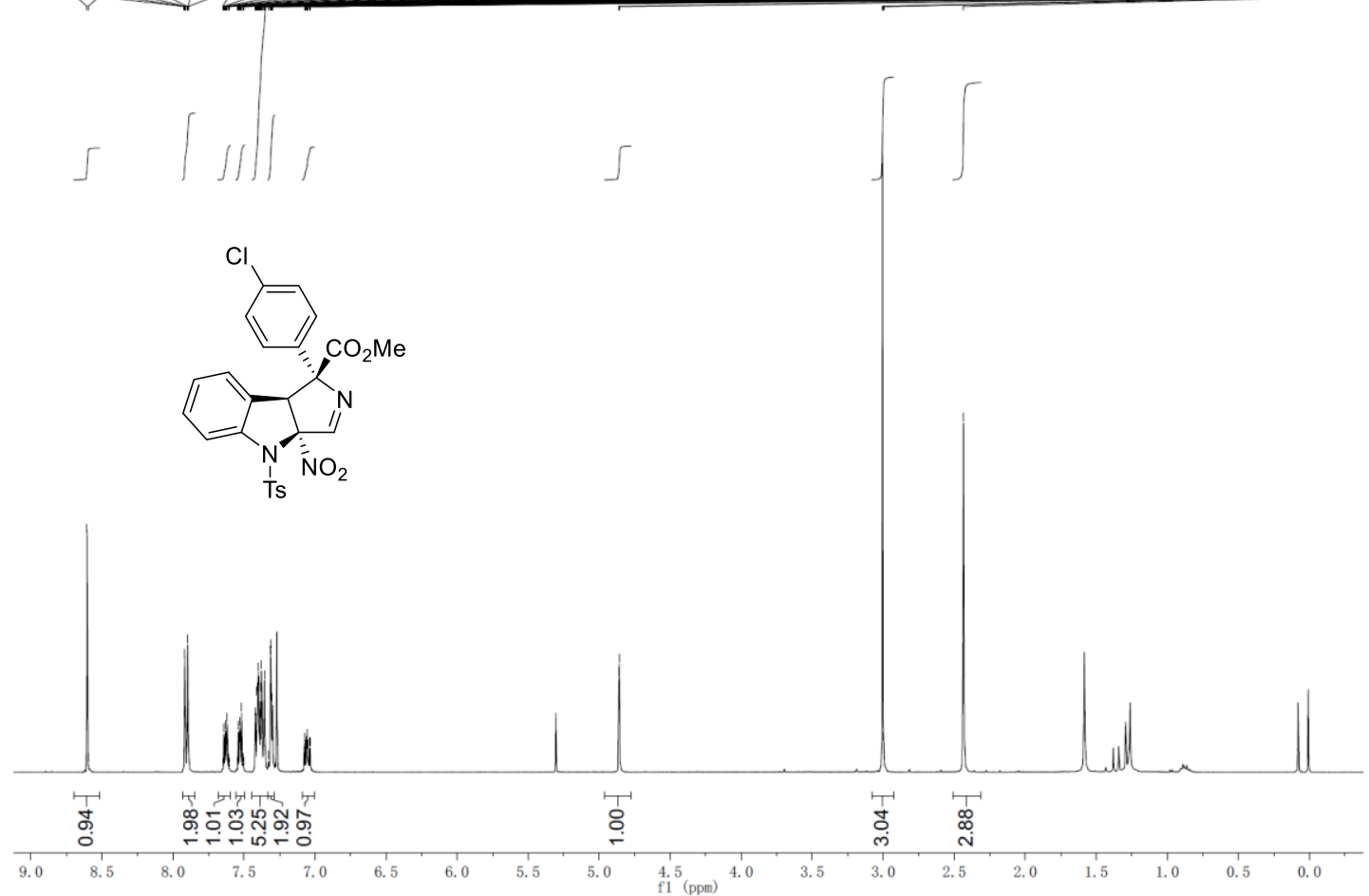

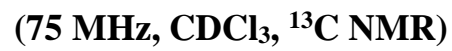

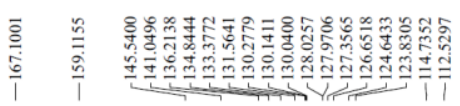

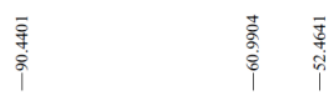

$\frac{\text { กิ }}{\frac{1}{2}}$<smiles>COC(=O)c1ccc(Cl)cc1</smiles>

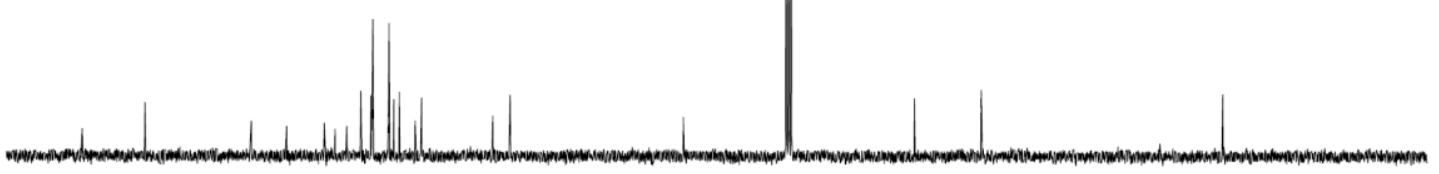

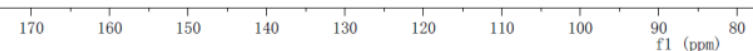




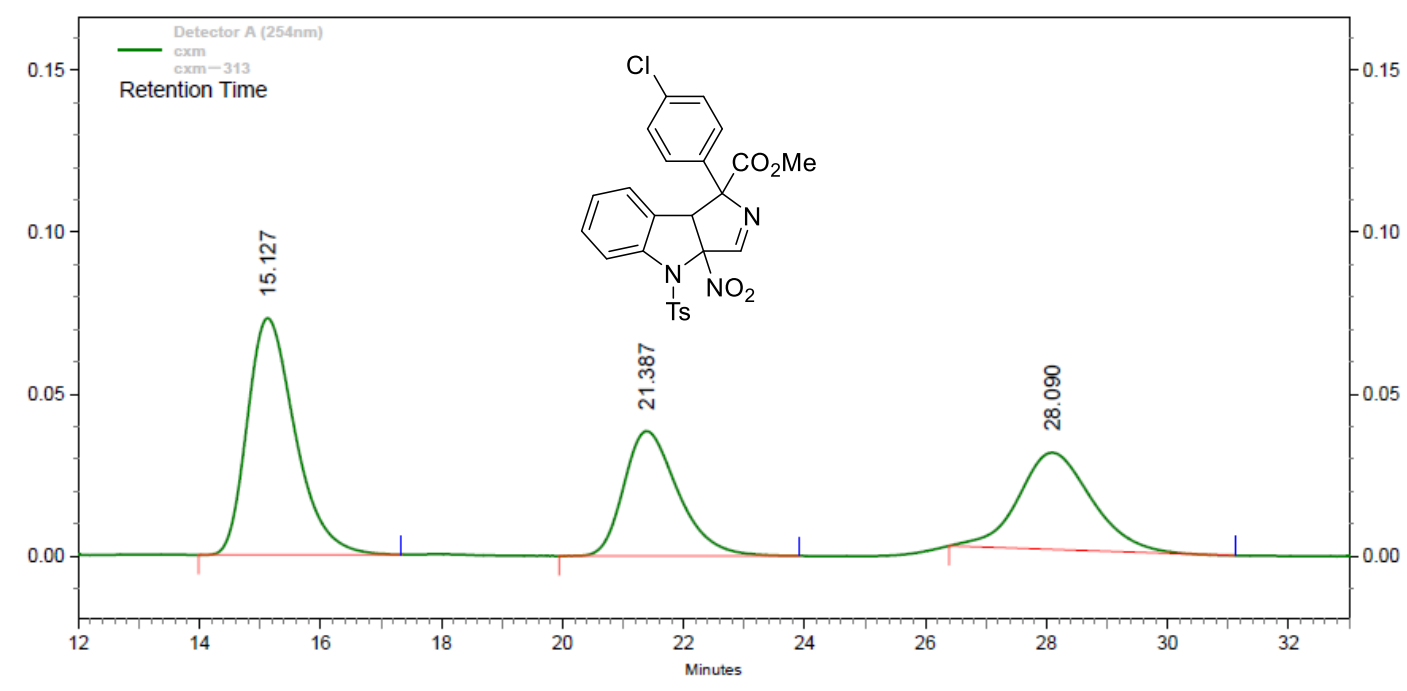

Detector

A

(254nm)

\begin{tabular}{rrrrrr} 
Pk \# & Retention Time & Height & Height Percent & Area & Area Percent \\
\hline 1 & 15.127 & 73035 & 51.66 & 4013124 & 44.98 \\
2 & 21.387 & 38486 & 27.22 & 2401018 & 26.91 \\
3 & 28.090 & 29842 & 21.11 & 2507838 & 28.11
\end{tabular}

\begin{tabular}{|r|r|r|rr|}
\hline Totals & 141363 & 100.00 & 8921980 & 100.00 \\
\hline
\end{tabular}

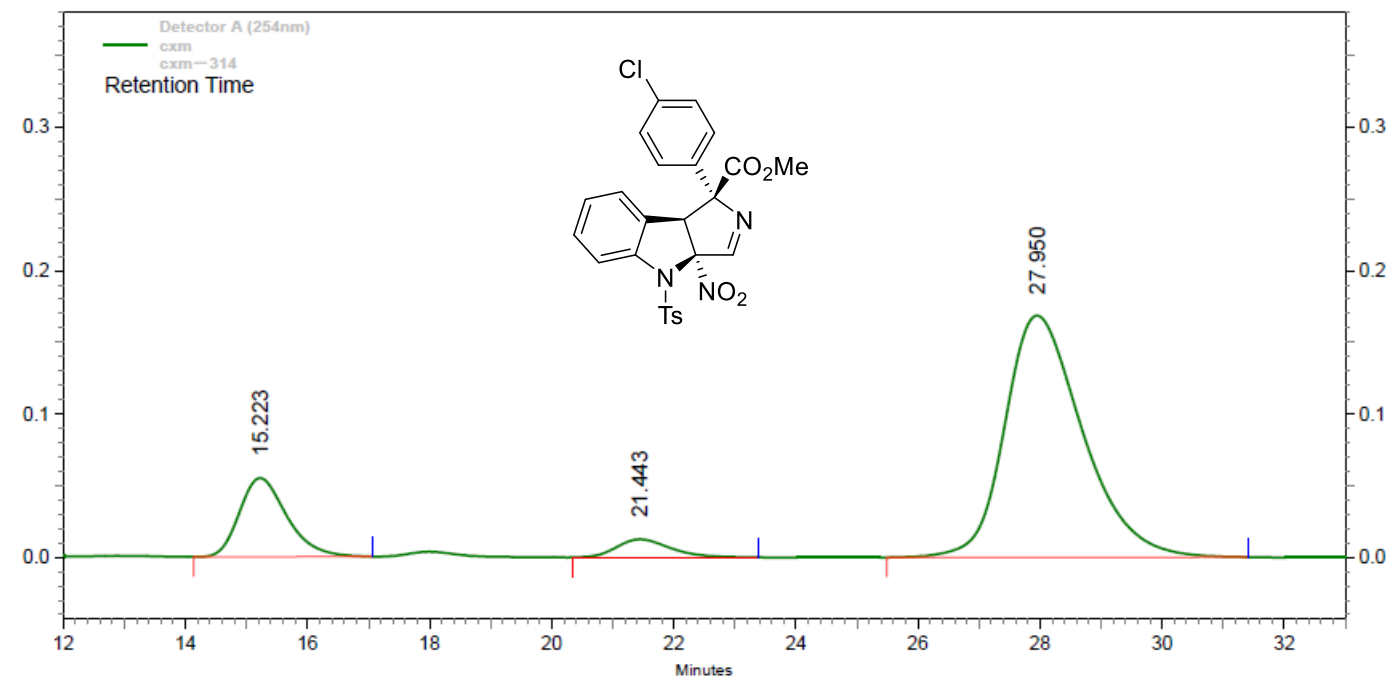

Detector

A

\begin{tabular}{rrrrrr}
$\begin{array}{r}\text { (254nm) } \\
\text { Pk \# }\end{array}$ & Retention Time & Height & Height Percent & Area & Area Percent \\
\hline 1 & 15.223 & 54885 & 23.25 & 2962947 & 15.86 \\
2 & 21.443 & 12548 & 5.32 & 765692 & 4.10 \\
3 & 27.950 & 168617 & 71.43 & 14954451 & 80.04
\end{tabular}

\begin{tabular}{|r|r|r|rr|}
\hline Totals & 236050 & 100.00 & 18683090 & 100.00 \\
\hline
\end{tabular}


${ }^{1} \mathrm{H}$ NMR, ${ }^{13} \mathrm{C}$ NMR and HPLC of 3n

(300 $\mathrm{MHz}, \mathrm{CDCl}_{3},{ }^{1} \mathrm{H}$ NMR)
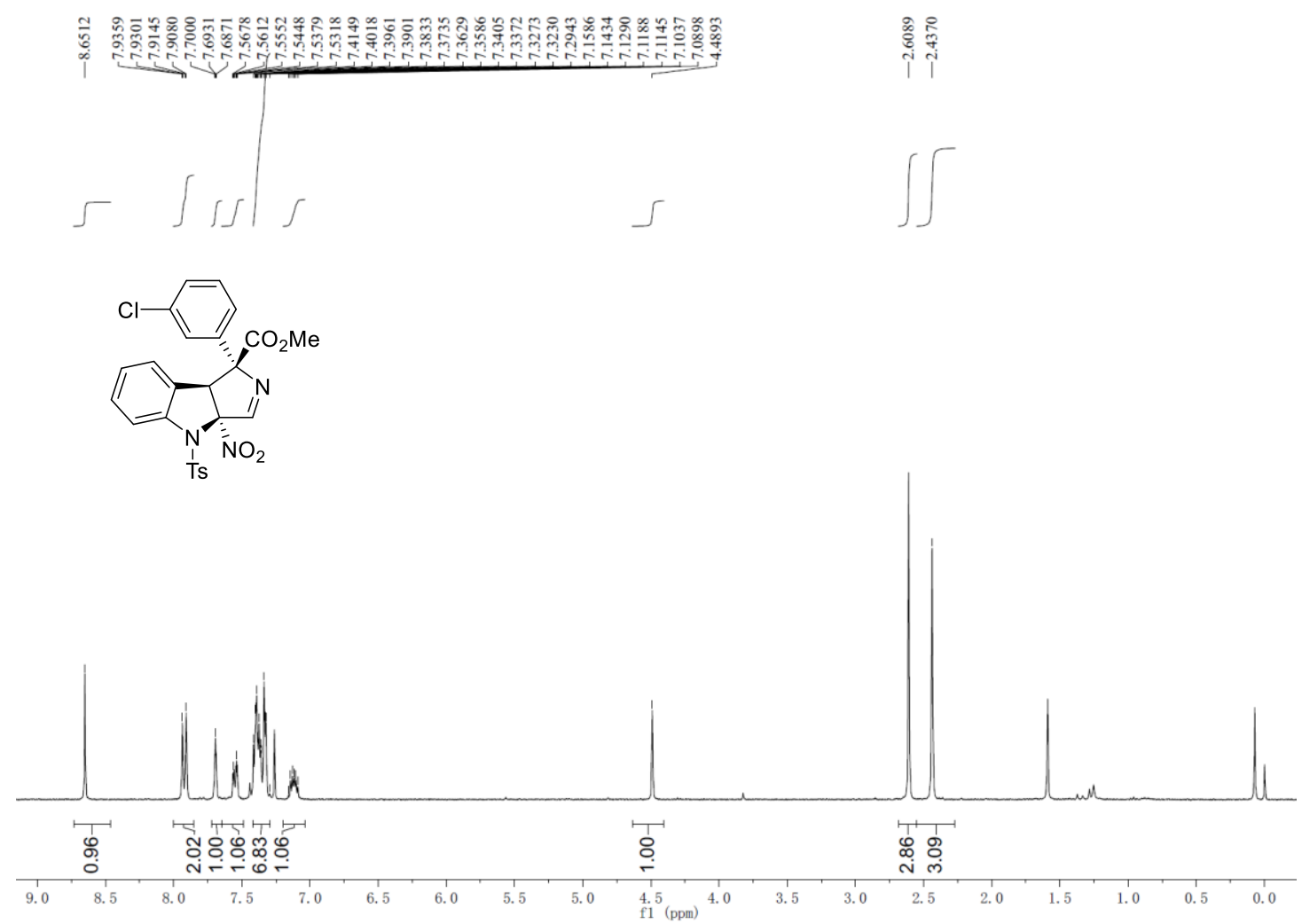

(75 MHz, $\mathrm{CDCl}_{3},{ }^{13} \mathrm{C}$ NMR)

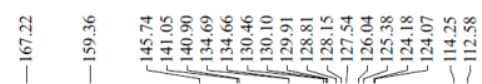

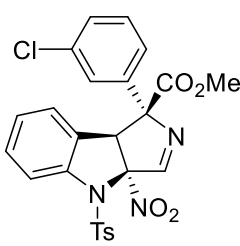

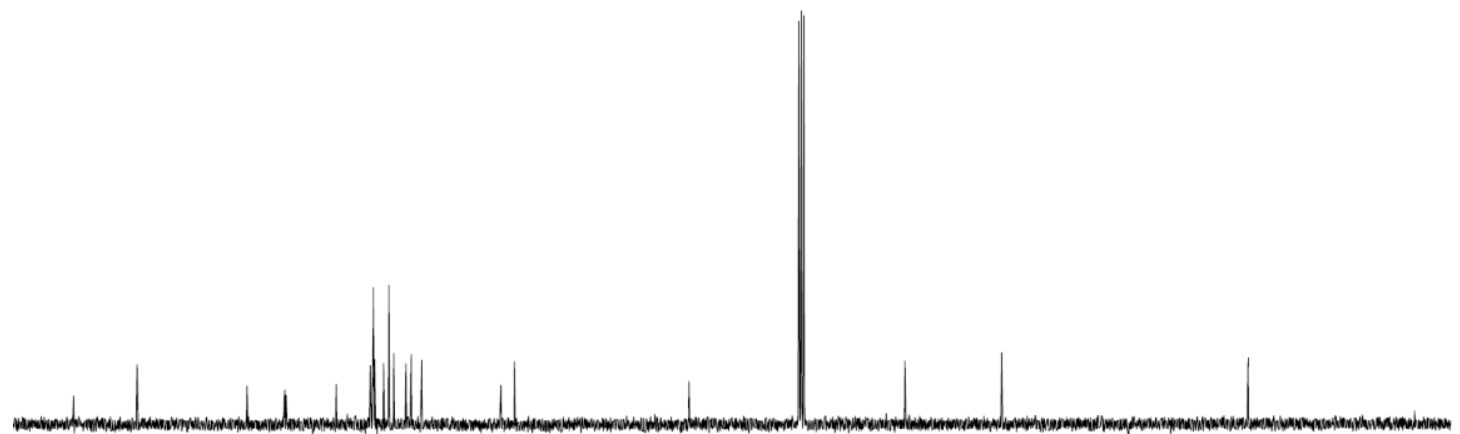




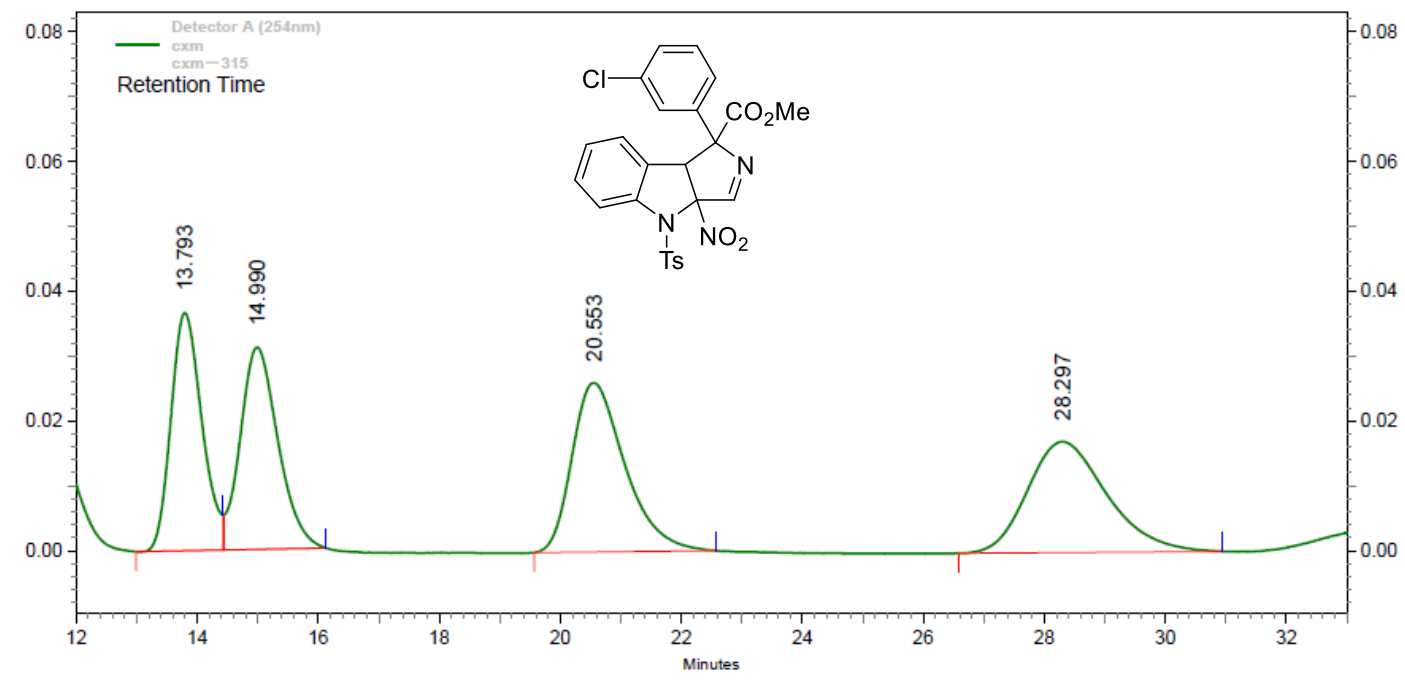

Detector

(254nm)

Pk \# Retention Time Height Height Percent $33.04 \quad 1299551 \quad 22.46$ 36657 28.06

23.50

15.40

23.33

20.553

26070

17091

27.33

28.297

110956

100.00

5785287

100.00

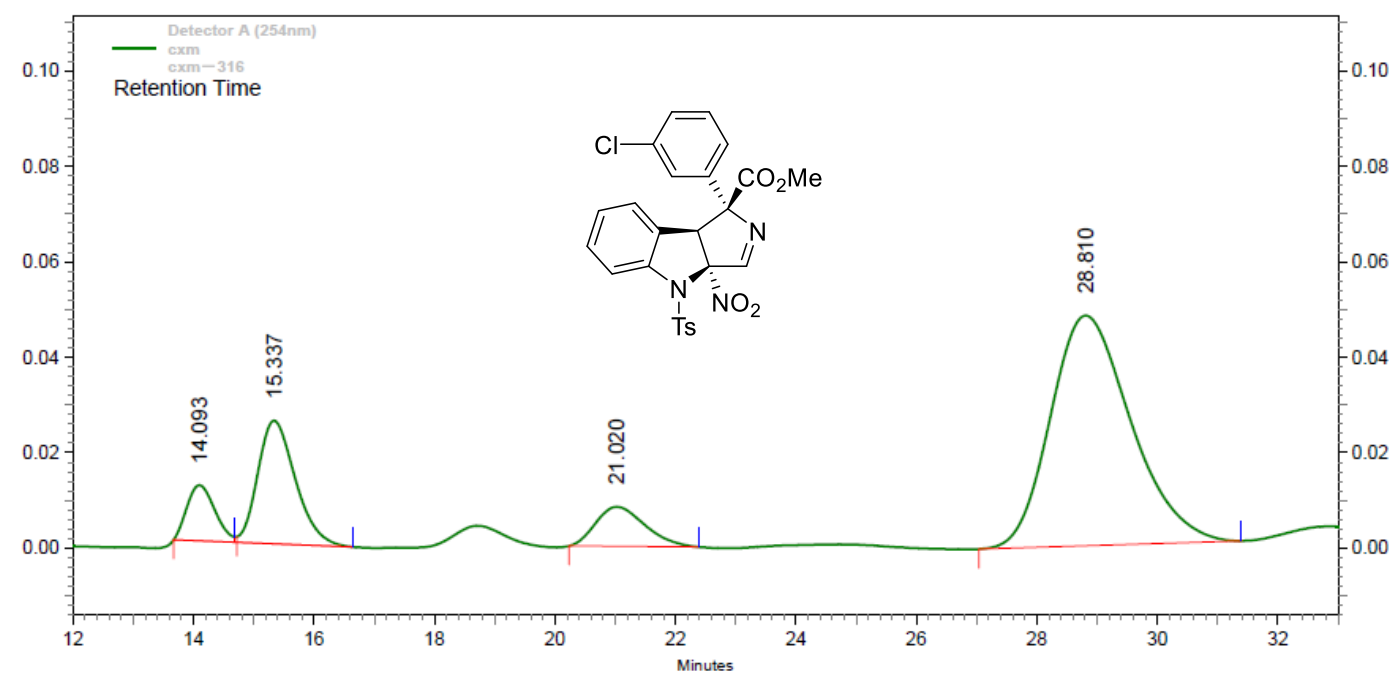

Detector

A

(254nm)

Pk \#

Retention Time

14.093

Height Height Percent

Are

11666

25778

8267

12.42
27.44

375075

Area Percent

21.020
28.810

48219

8.80
51.34

1118553

468414

17.39

4471212

69.50

\begin{tabular}{|r|r|r|rr|}
\hline Totals & 93930 & 100.00 & 6433254 & 100.00 \\
\hline
\end{tabular}


${ }^{1} \mathrm{H}$ NMR, ${ }^{13} \mathrm{C}$ NMR and HPLC of 30

(300 MHz, $\mathrm{CDCl}_{3},{ }^{1} \mathrm{H}$ NMR)
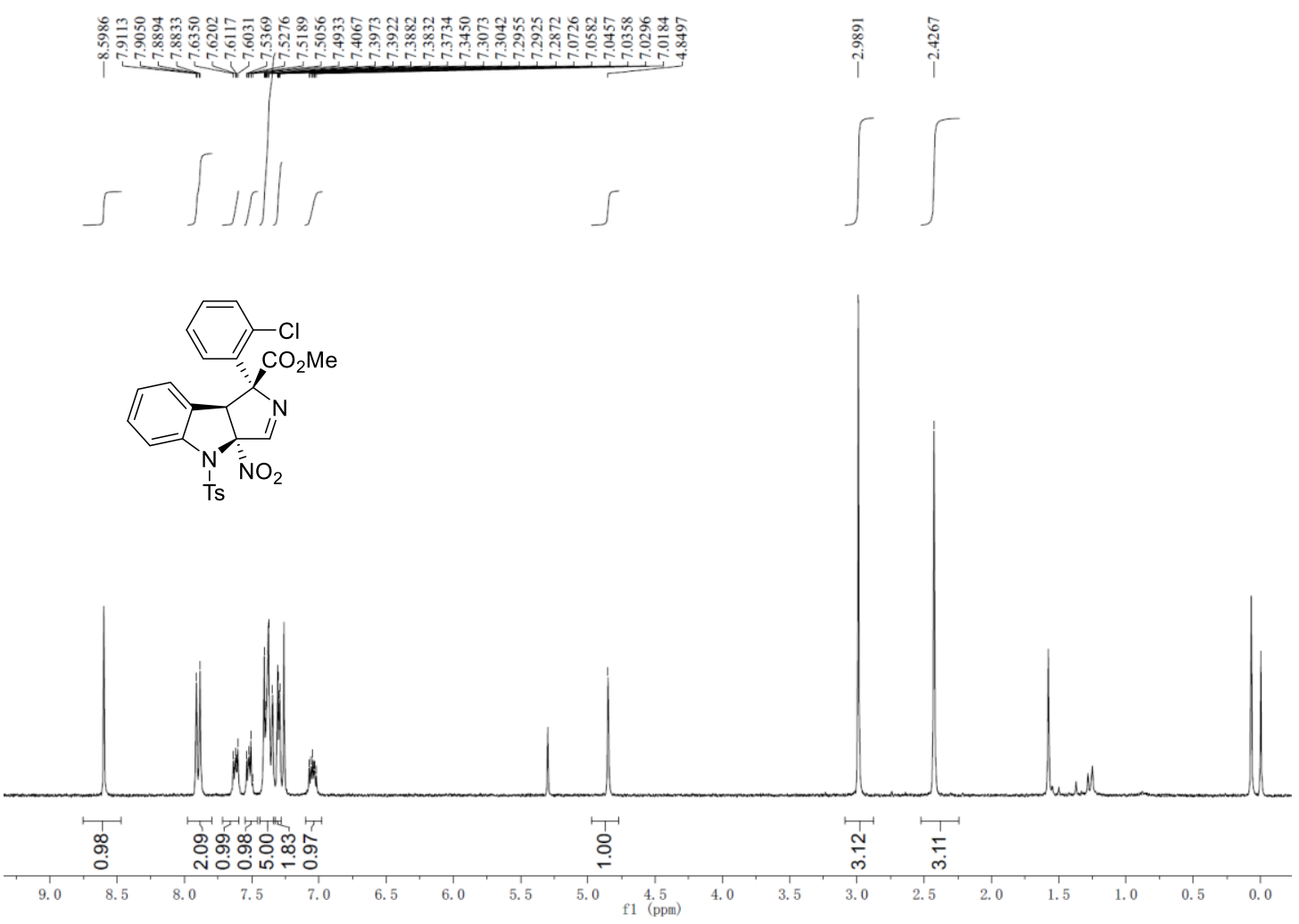

(75 $\mathrm{MHz}, \mathrm{CDCl}_{3},{ }^{13} \mathrm{C}$ NMR)

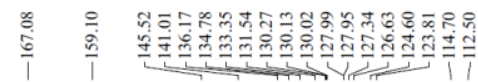
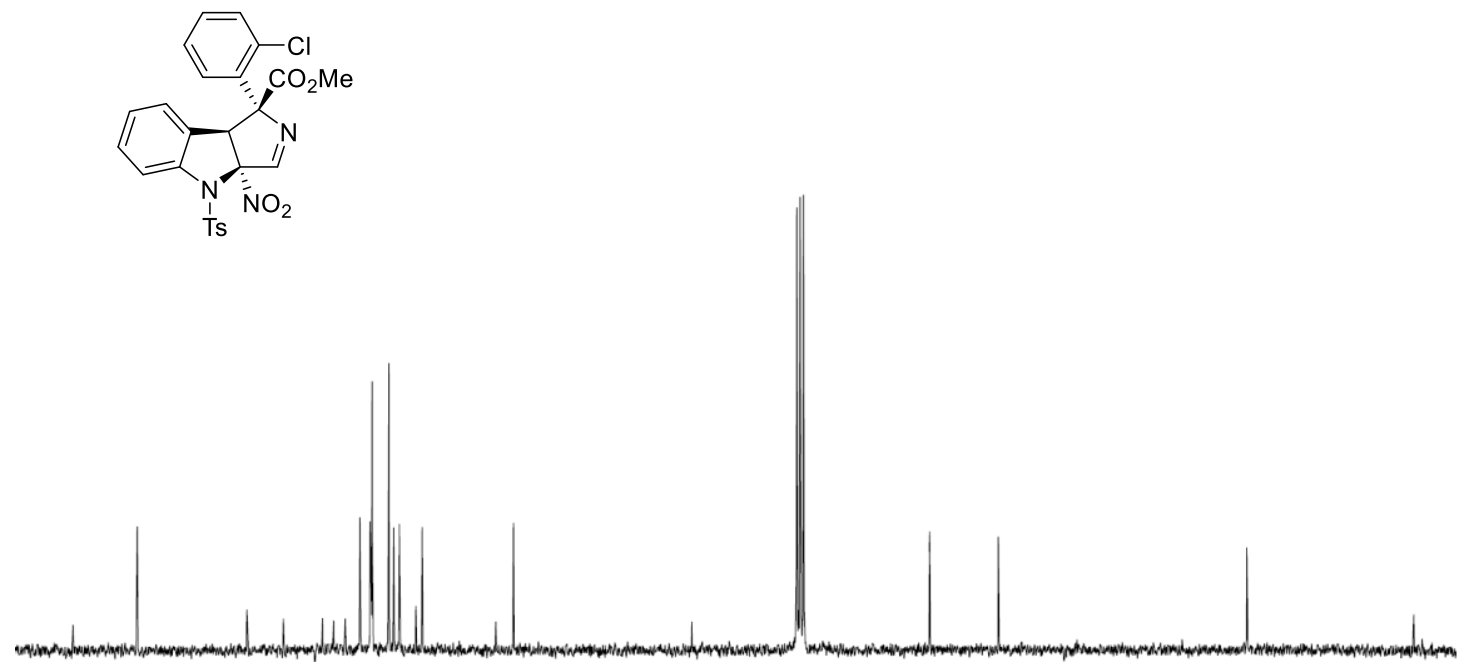

$\begin{array}{lllllllll}170 & 160 & 150 & 140 & 130 & 120 & 110 & 100 & 90 \quad \text { f1 (ppm) }\end{array}$ 


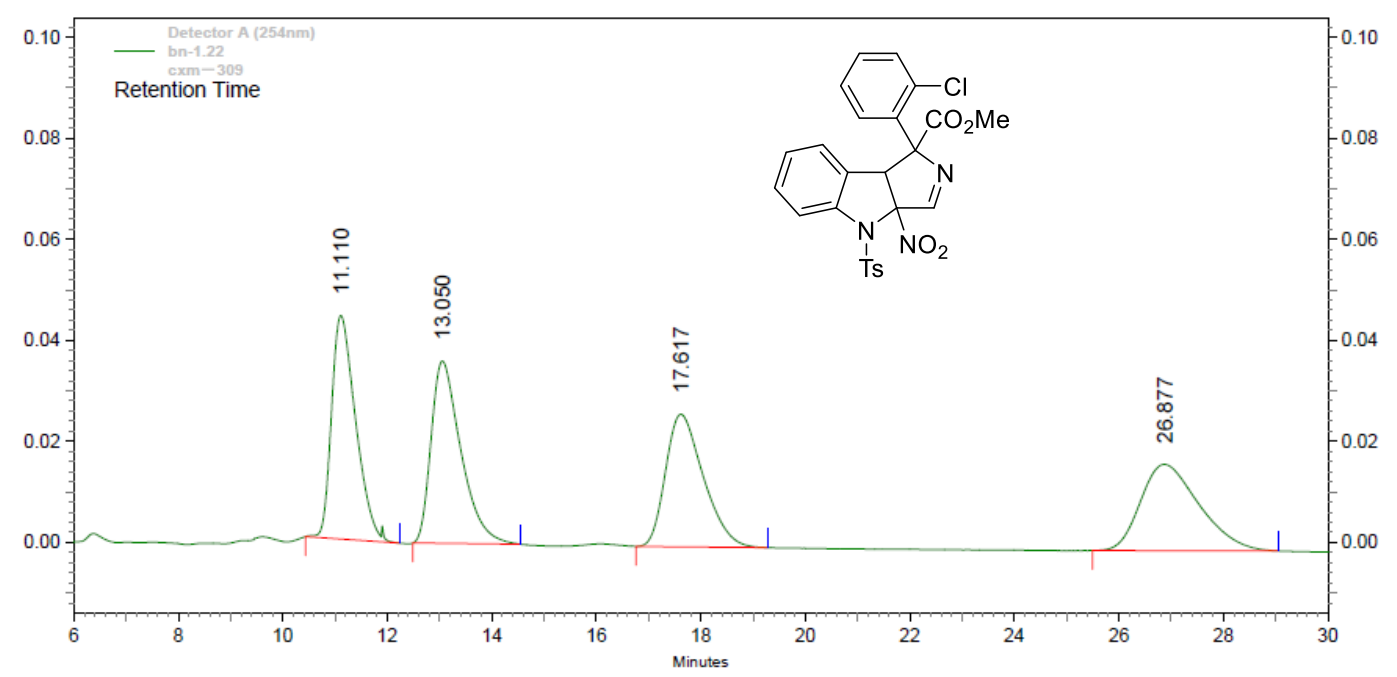

Detector

A

(254nm)

\begin{tabular}{rrrrrr}
$\begin{array}{r}(254 n m) \\
\text { Pk \# }\end{array}$ & Retention Time & Height & Height Percent & Area & Area Percent \\
\hline 1 & 11.110 & 44226 & 35.79 & 1420759 & 25.86 \\
2 & 13.050 & 36046 & 29.17 & 1428409 & 25.99 \\
3 & 17.617 & 26224 & 21.22 & 1317582 & 23.98 \\
4 & 26.877 & 17074 & 13.82 & 1328231 & 24.17
\end{tabular}

\begin{tabular}{|r|r|r|rr|}
\hline Totals & 123570 & 100.00 & 5494981 & 100.00 \\
\hline
\end{tabular}

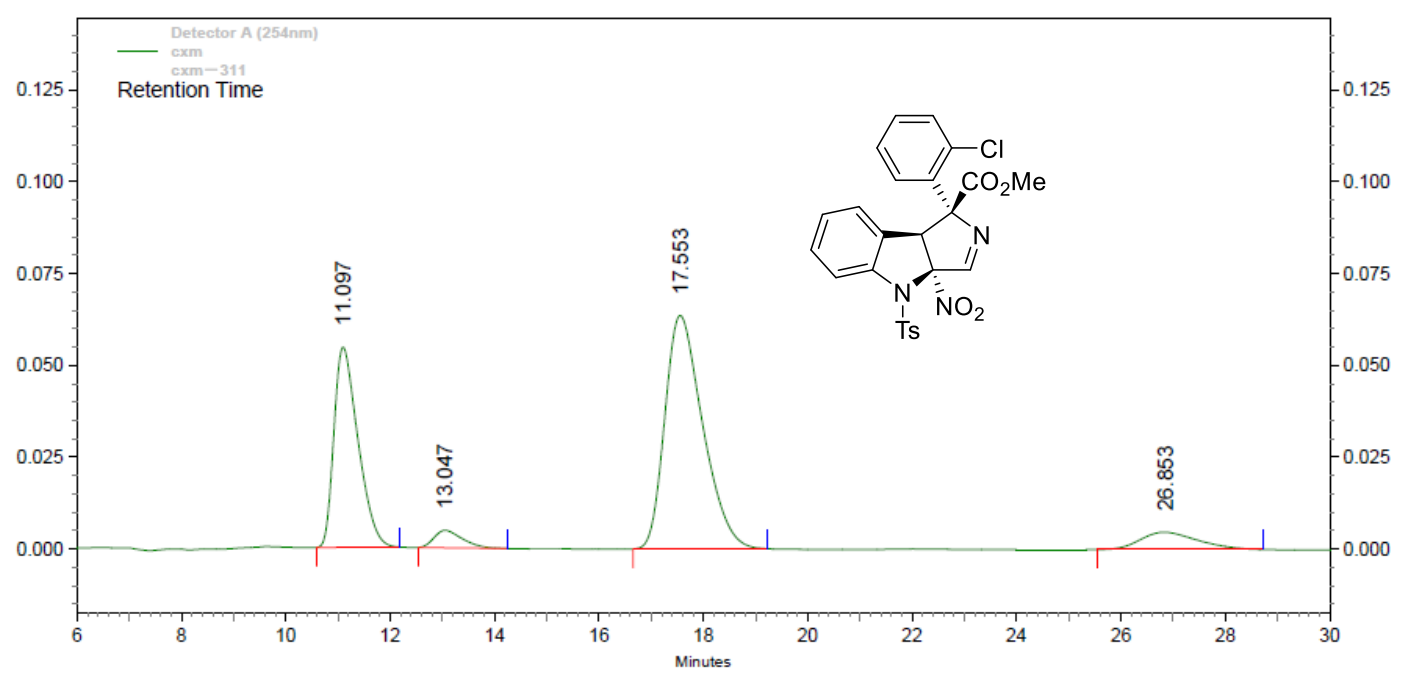

Detector

A

(254nm)

\begin{tabular}{rrrrrr} 
Pk \# & Retention Time & Height & Height Percent & Area & Area Percent \\
\hline 1 & 11.097 & 54490 & 42.75 & 1751308 & 31.90 \\
2 & 13.047 & 4739 & 3.72 & 179146 & 3.26 \\
3 & 17.553 & 63526 & 49.84 & 3201065 & 58.32 \\
4 & 26.853 & 4694 & 3.68 & 357695 & 6.52
\end{tabular}

\begin{tabular}{|r|r|r|rr|}
\hline Totals & 127449 & 100.00 & 5489214 & 100.00 \\
& & 1274 & & \\
\hline
\end{tabular}


${ }^{1} \mathrm{H}$ NMR, ${ }^{13} \mathrm{C}$ NMR and HPLC of 3p

(300 $\mathrm{MHz}, \mathrm{CDCl}_{3},{ }^{1} \mathrm{H}$ NMR)

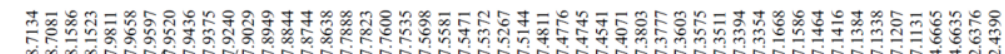

作
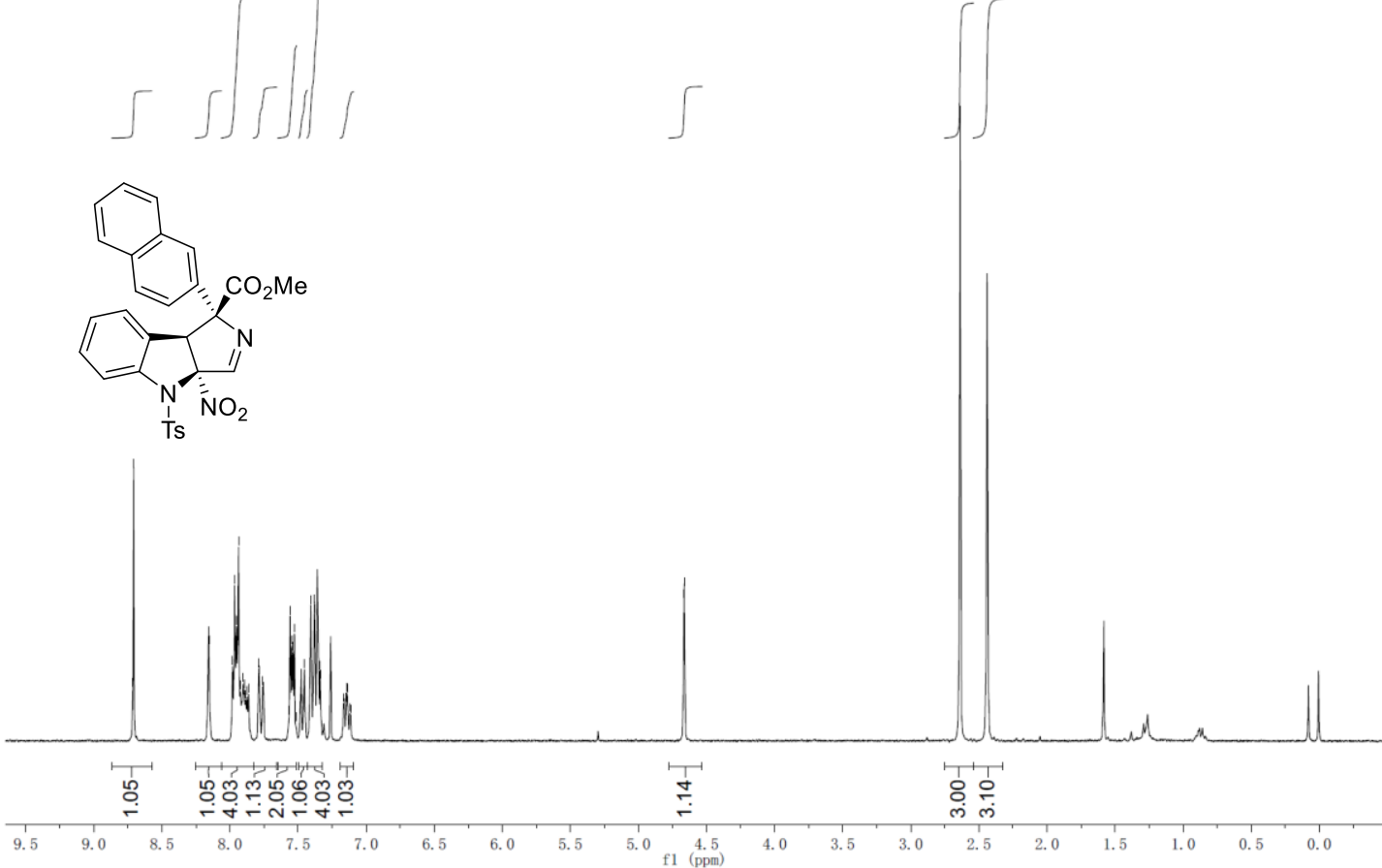

$\left(75 \mathrm{MHz}, \mathrm{CDCl}_{3},{ }^{13} \mathrm{C} \mathrm{NMR}\right)$

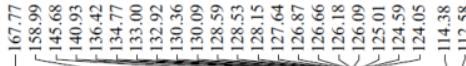

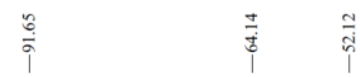

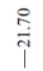

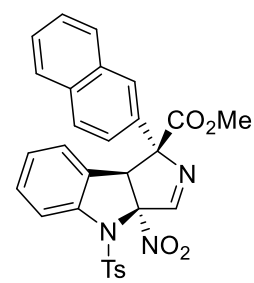

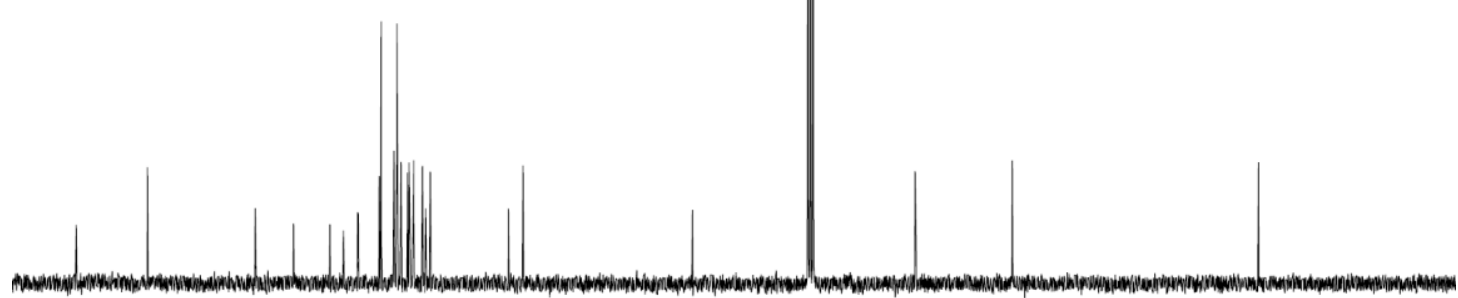

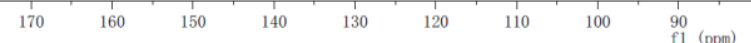




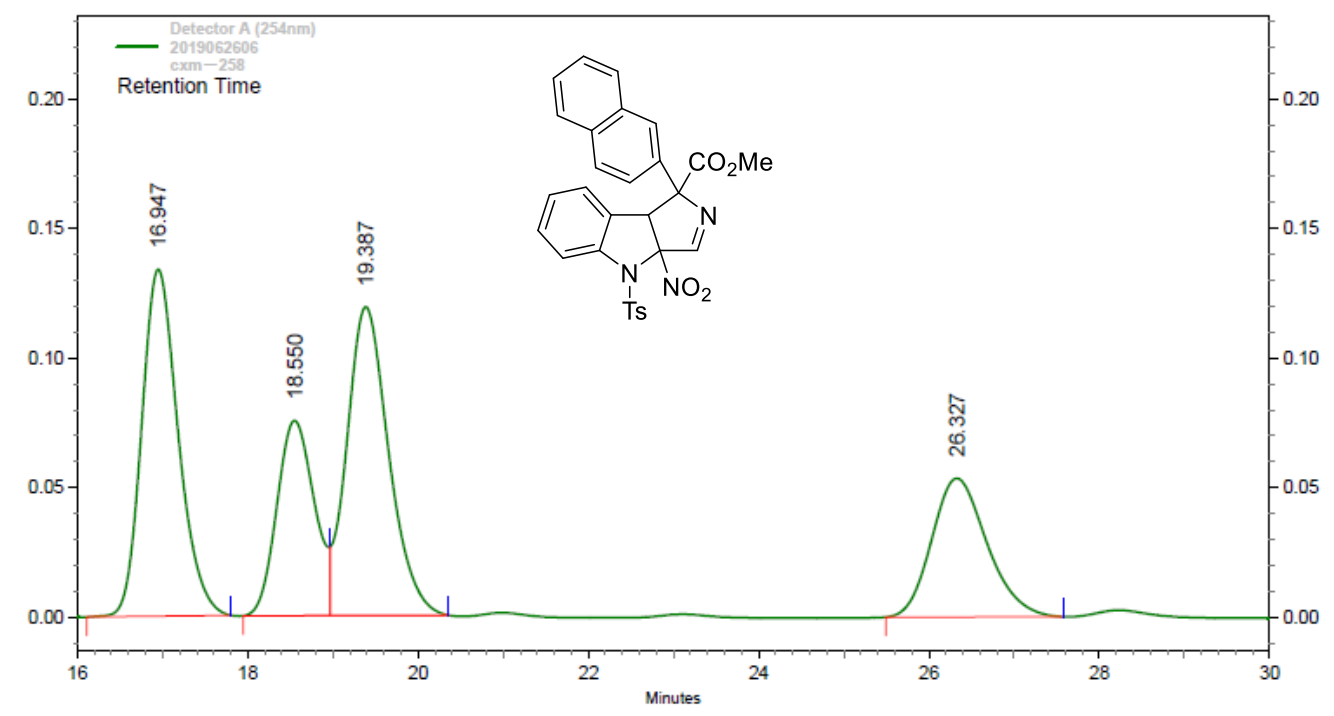

Detector

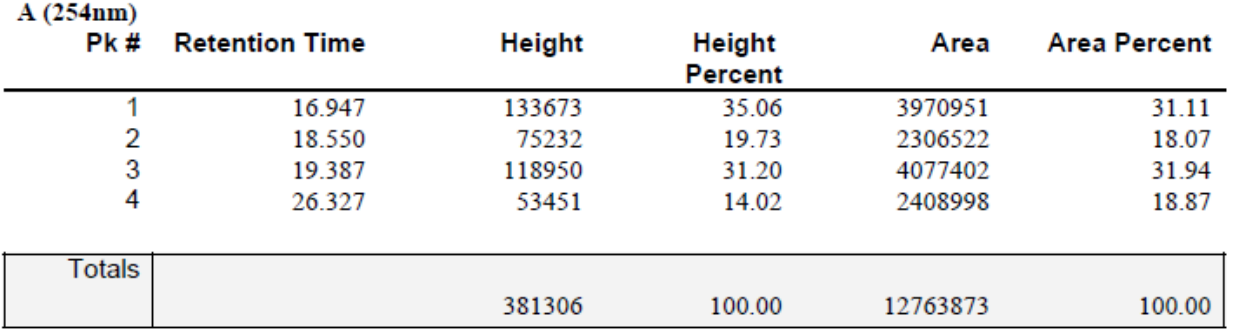

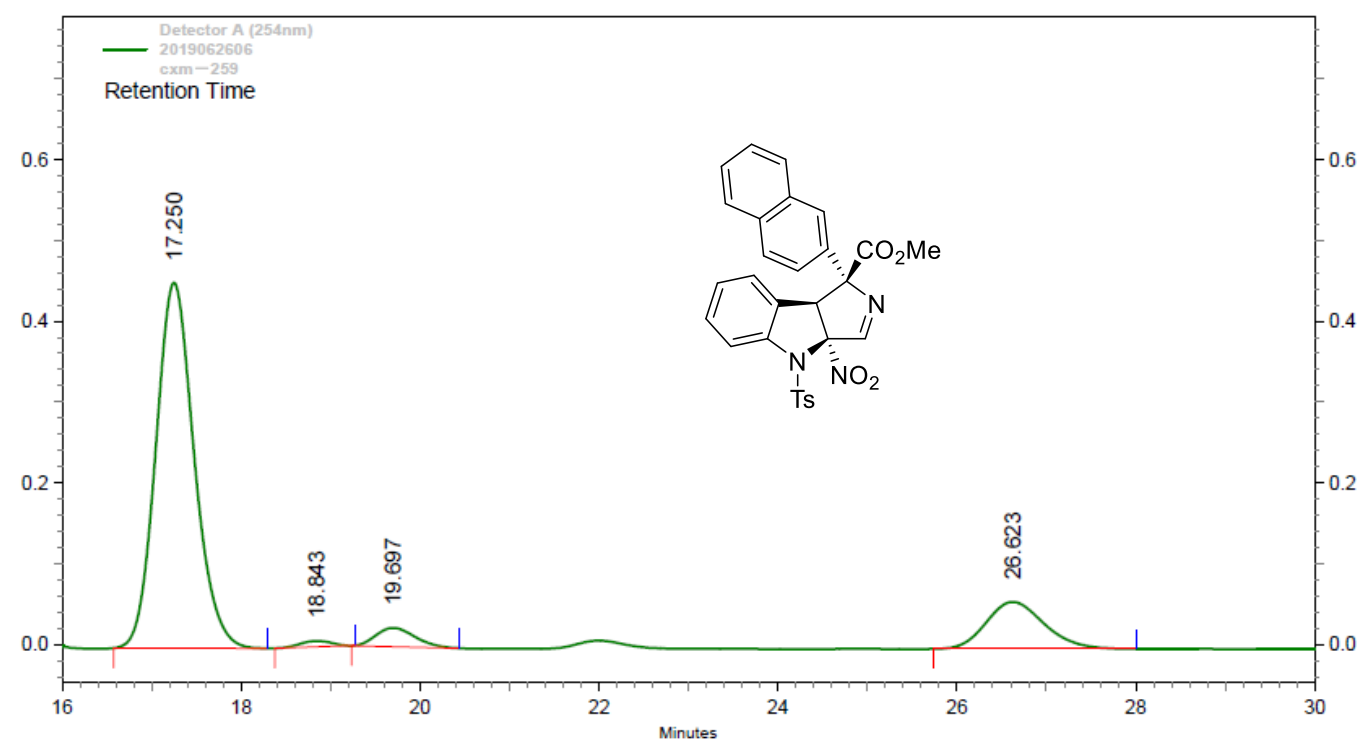

Detector

A (254nm)

Pk \# Retention Time

Height

Height

Area Area Percent

$\begin{array}{ll}1 & 17.250\end{array}$

452462

Percent

79.22

$2 \quad 18.843$

19.697

7367

23419

83.55

1.05

$26.623 \quad 58305$

4.32

713732

4.21

10.77

2633591

15.52

\begin{tabular}{|c|c|c|c|c|}
\hline \multirow[t]{2}{*}{ Totals } & & & & \\
\hline & 541553 & 100.00 & 16966798 & 100.00 \\
\hline
\end{tabular}


${ }^{1} \mathrm{H}$ NMR, ${ }^{13} \mathrm{C}$ NMR and HPLC of $3 q$

(300 $\mathrm{MHz}, \mathrm{CDCl}_{3},{ }^{1} \mathrm{H}$ NMR)

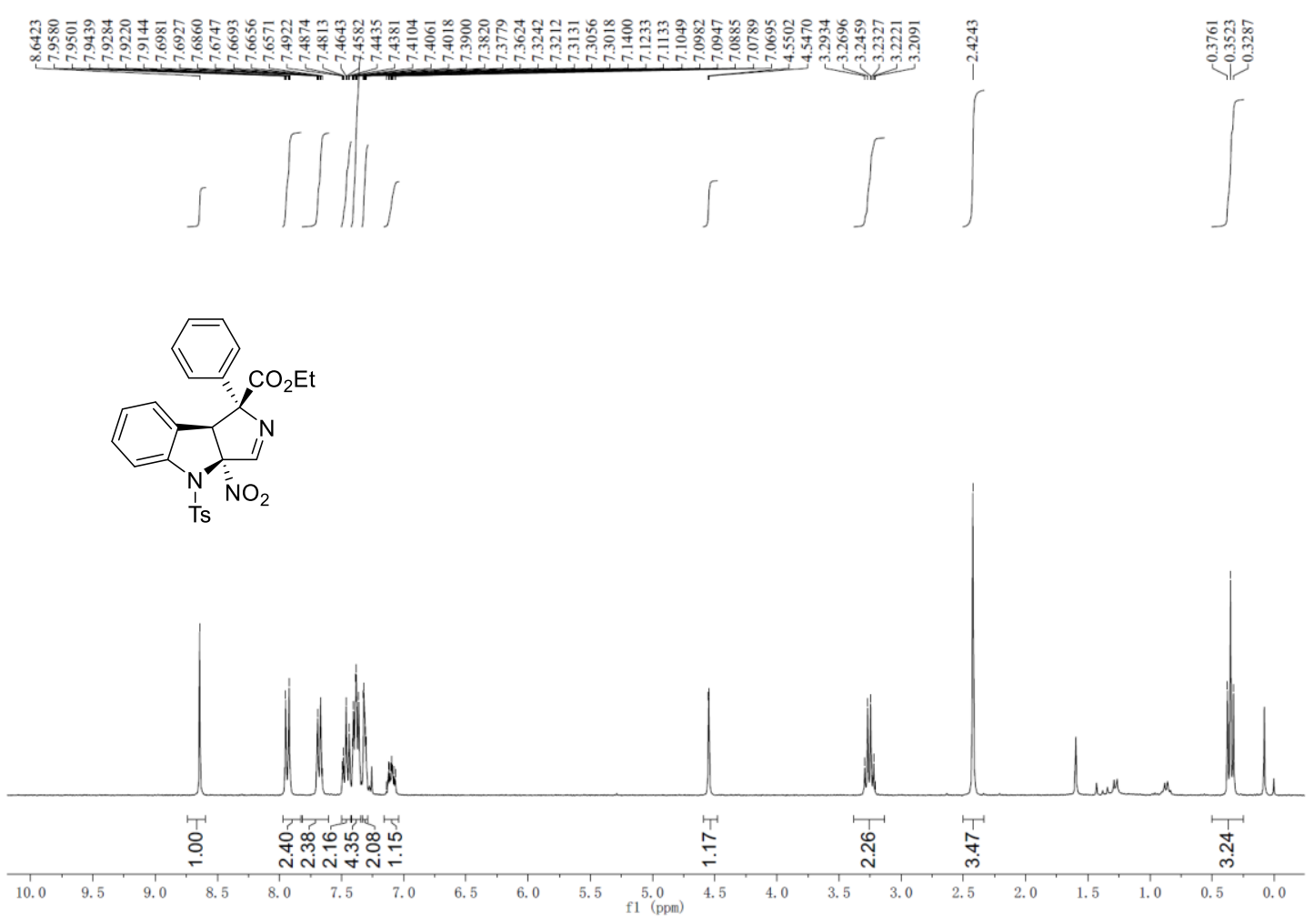

(75 MHz, $\left.\mathrm{CDCl}_{3},{ }^{13} \mathrm{C} \mathrm{NMR}\right)$

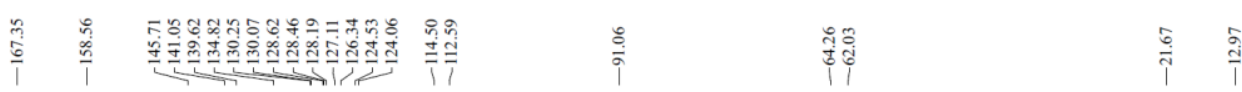
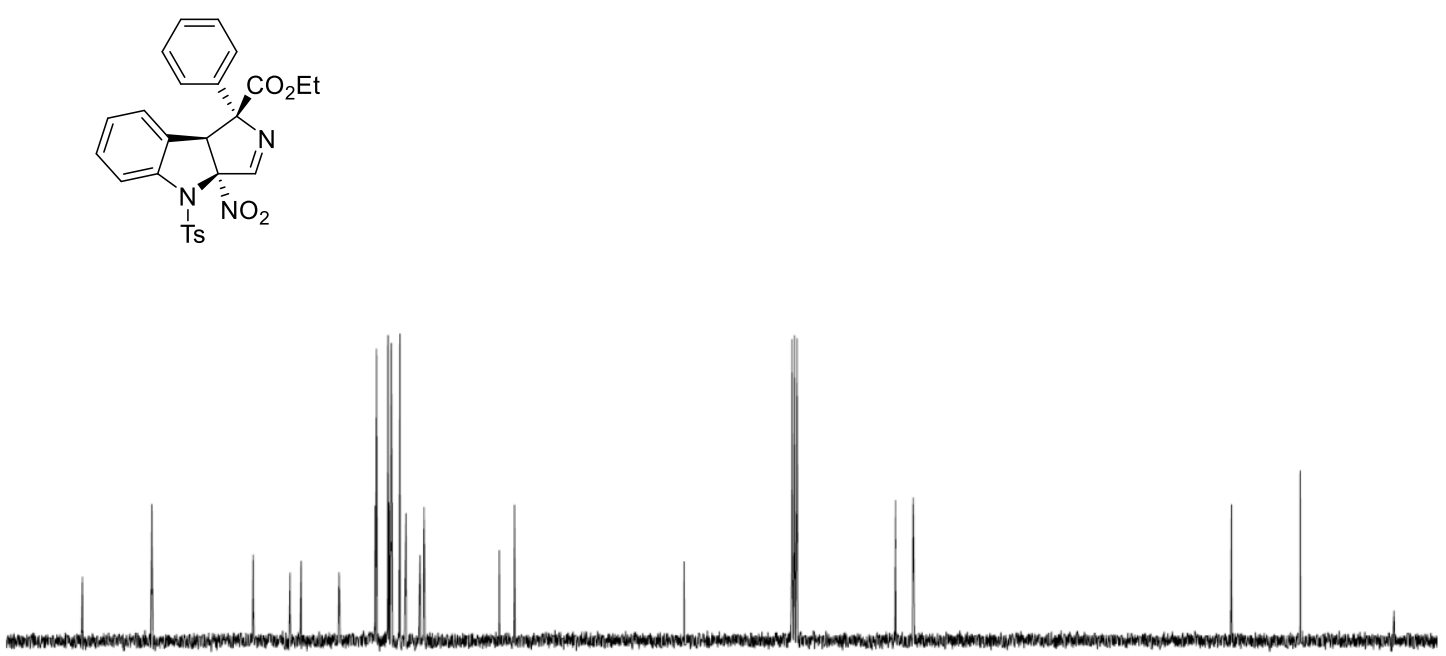

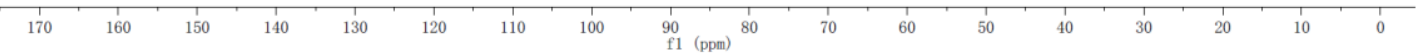


$\mathrm{mV}$

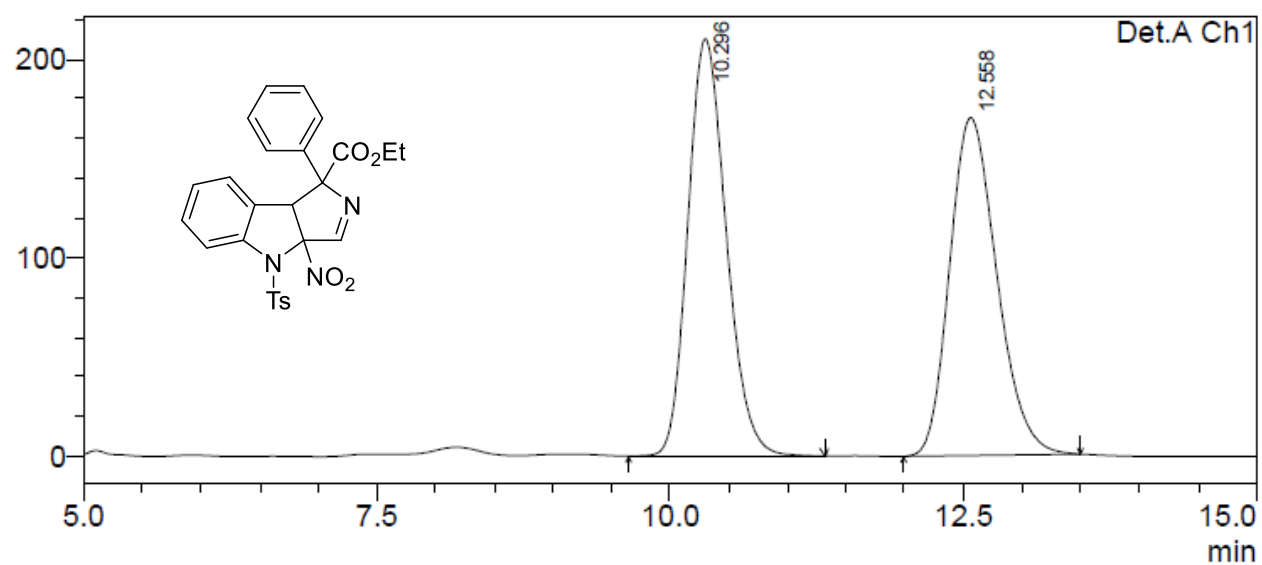

1 Det.A Ch1/254nm

PeakTable
Detector A Ch1 $254 \mathrm{~nm}$
\begin{tabular}{|c|c|c|c|c|}
\hline Peak\# & Ret. Time & Area & Height & Area $\%$ \\
\hline 1 & 10.296 & 4775187 & 209952 & 50.139 \\
\hline 2 & 12.558 & 4748664 & 169975 & 49.861 \\
\hline Total & & 9523852 & & 100.000 \\
\hline
\end{tabular}

$\mathrm{mV}$

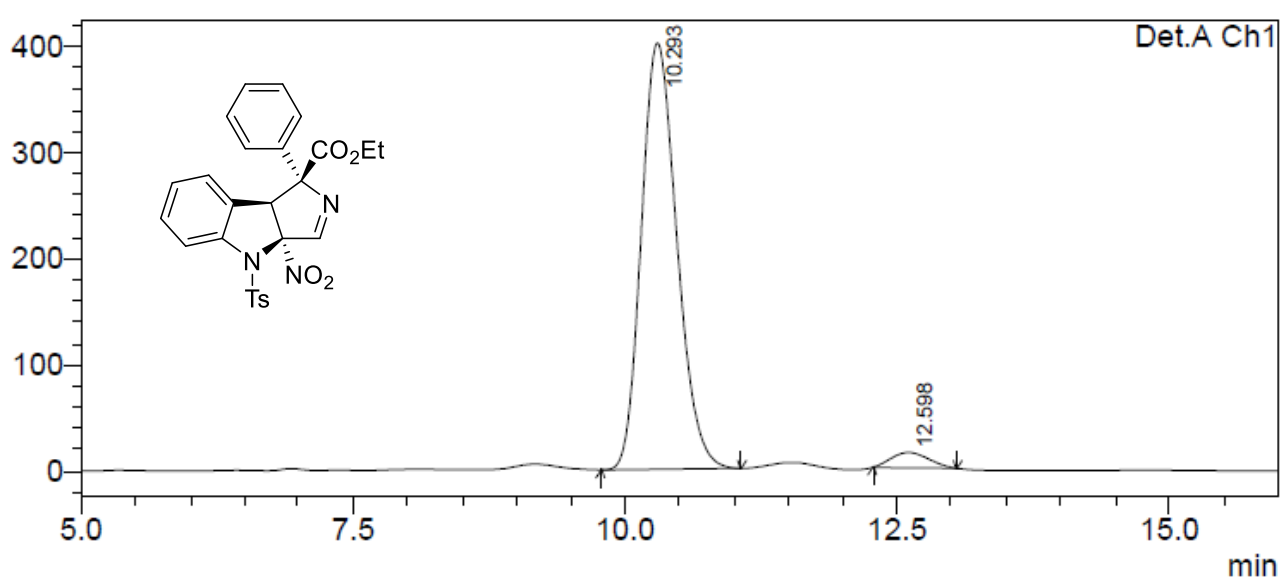

1 Det.A Ch $1 / 254 \mathrm{~nm}$

PeakTable

Detector A Ch1 254nm

\begin{tabular}{|c|c|c|c|c|}
\hline Peak\# & Ret. Time & Area & Height & Area \% \\
\hline 1 & 10.293 & 9144259 & 402558 & 96.317 \\
\hline 2 & 12.598 & 349692 & 14434 & 3.683 \\
\hline Total & & 9493950 & & 100.000 \\
\hline
\end{tabular}


${ }^{1} \mathrm{H}$ NMR, ${ }^{13} \mathrm{C}$ NMR and HPLC of $3 \mathrm{r}$

(300 $\mathrm{MHz}, \mathrm{CDCl}_{3},{ }^{1} \mathrm{H}$ NMR)

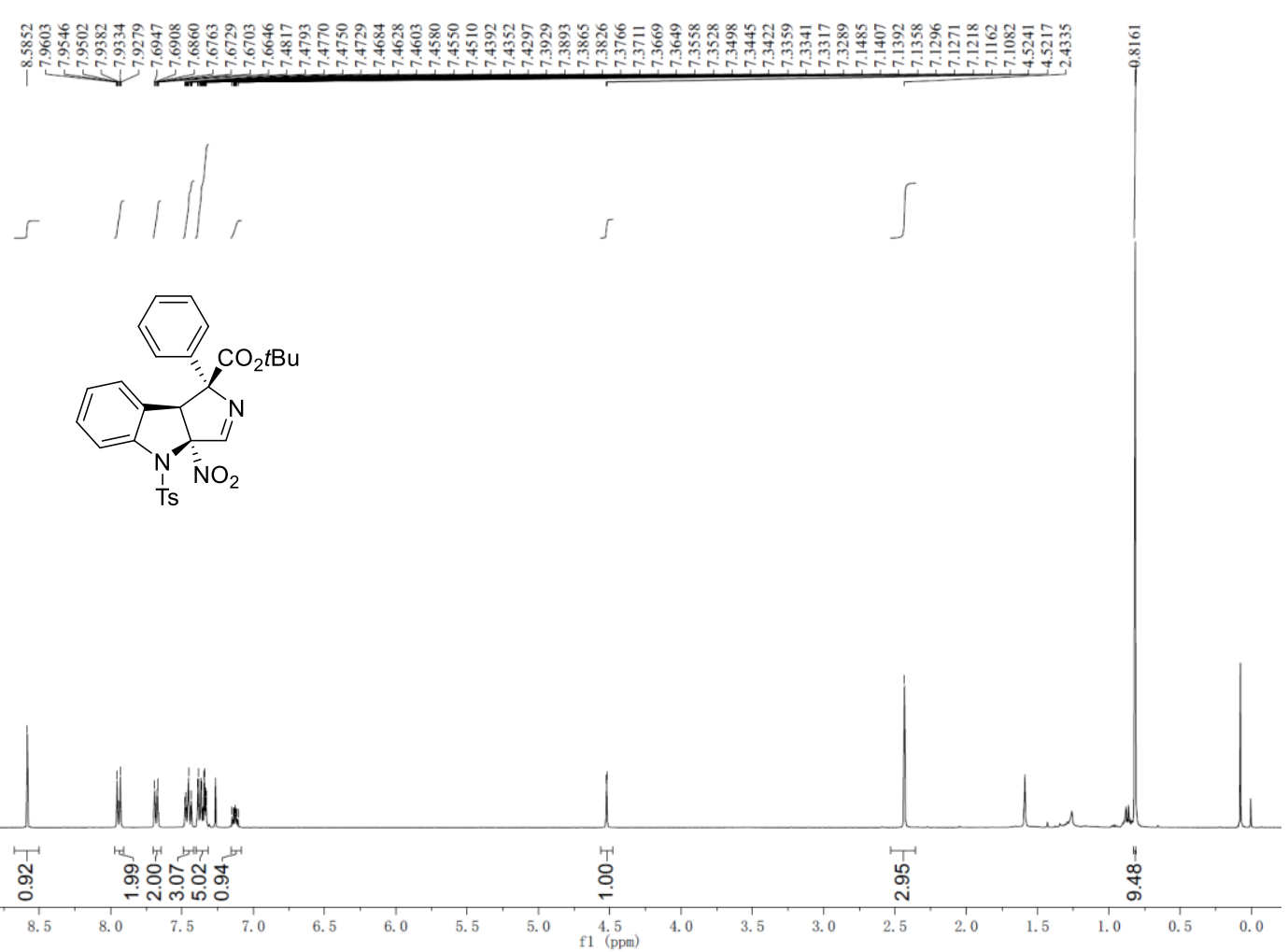

(75 $\left.\mathrm{MHz}, \mathrm{CDCl}_{3},{ }^{13} \mathrm{C} \mathrm{NMR}\right)$
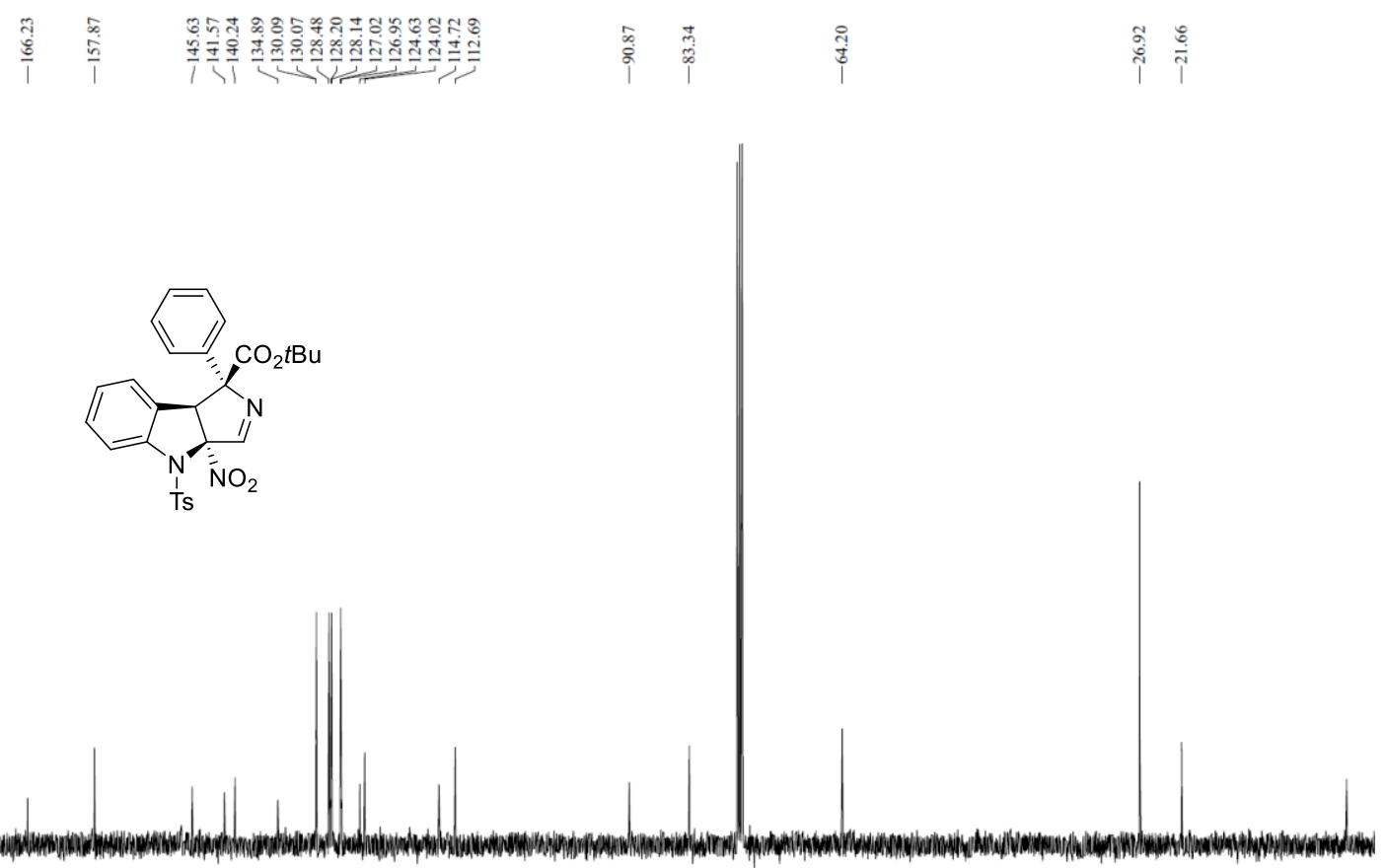

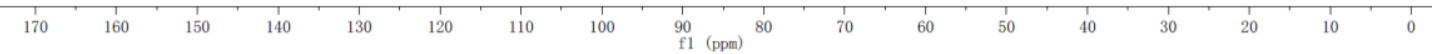




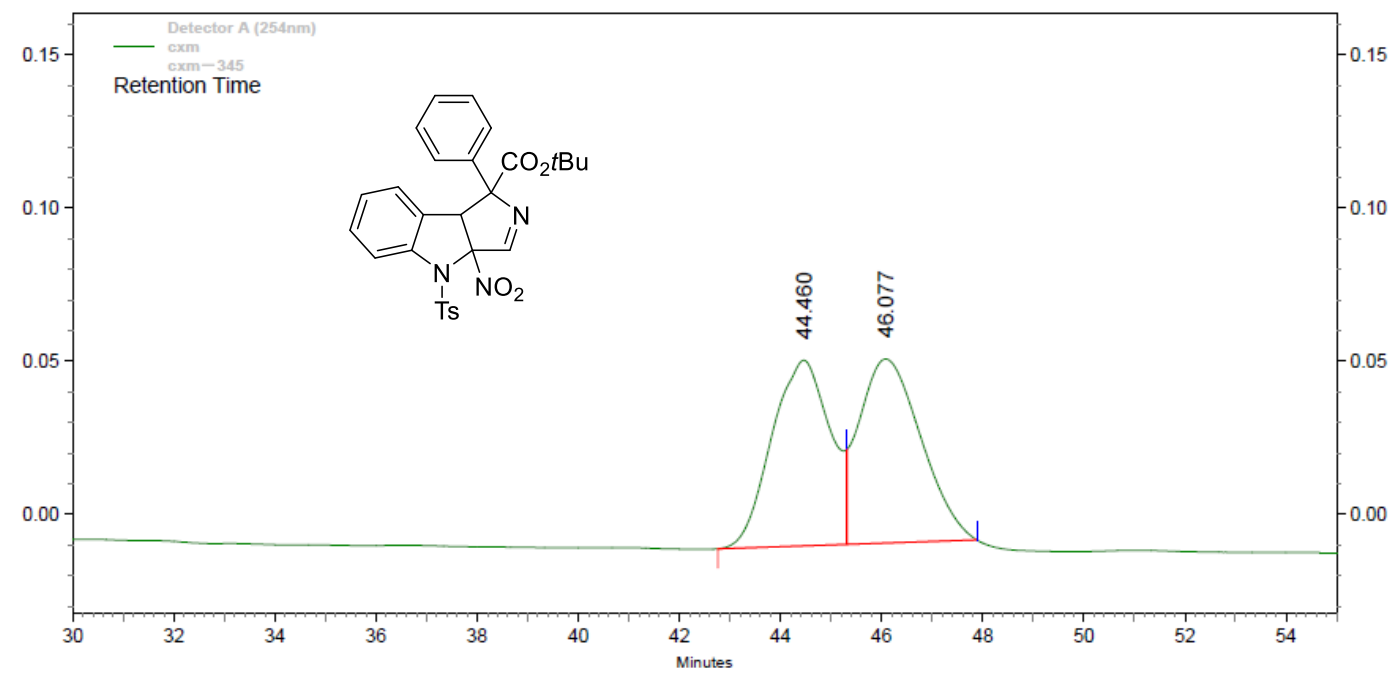

Detector

A

(254nm)

\begin{tabular}{rrrrrr} 
Pk \# & Retention Time & Height & Height Percent & Area & Area Percent \\
\hline 1 & 44.460 & 60567 & 50.21 & 4876862 & 48.76 \\
2 & 46.077 & 60066 & 49.79 & 5125458 & 51.24
\end{tabular}

\begin{tabular}{|r|r|r|rr|}
\hline Totals & 120633 & 100.00 & 10002320 & 100.00 \\
\hline
\end{tabular}

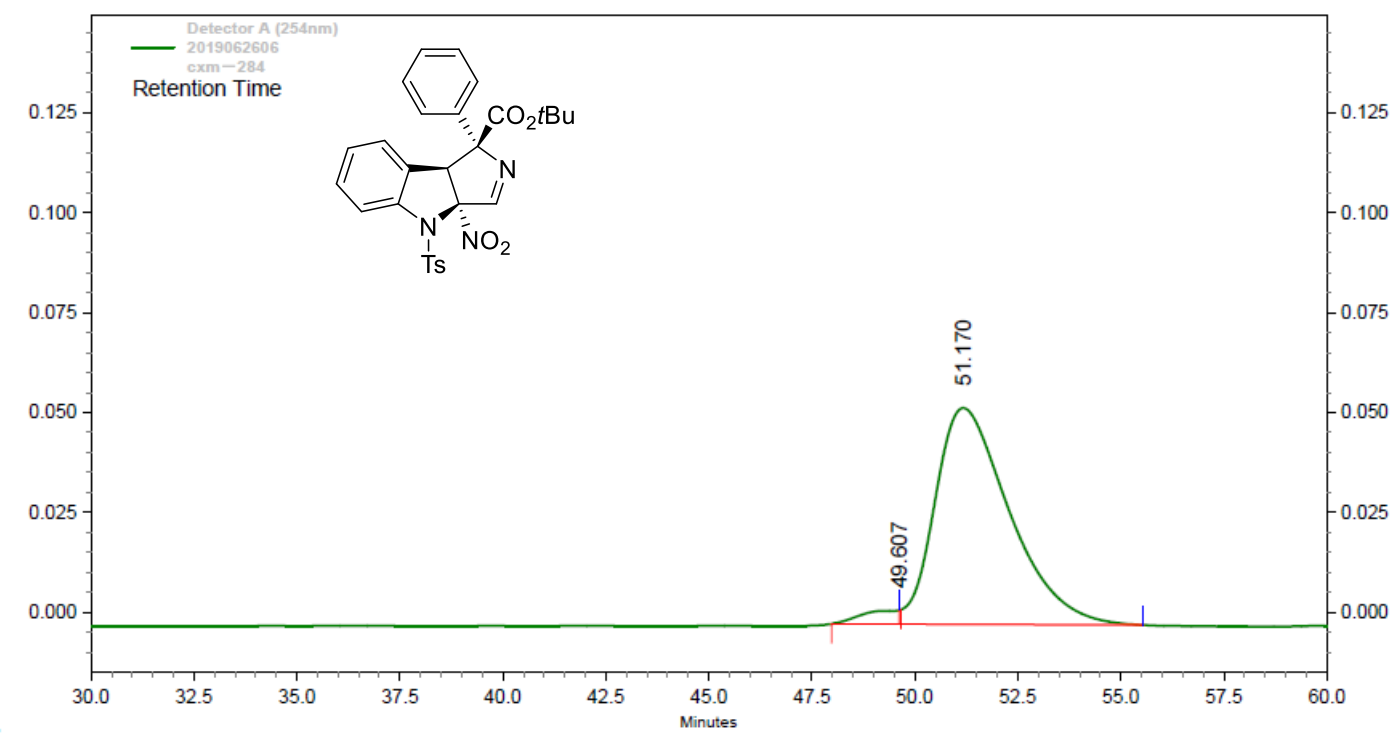

Detector

\begin{tabular}{|c|c|c|c|c|c|}
\hline \multicolumn{6}{|l|}{ A (254nm) } \\
\hline $\mathrm{Pk} \#$ & Retention Time & Height & $\begin{array}{l}\text { Height } \\
\text { Percent }\end{array}$ & Area & Area Percent \\
\hline 1 & 49.607 & 3454 & 6.00 & 214160 & 3.05 \\
\hline 2 & 51.170 & 54133 & 94.00 & 6806889 & 96.95 \\
\hline
\end{tabular}

\begin{tabular}{|c|c|c|c|c|}
\hline Totals & & & & \\
\hline & 57587 & 100.00 & 7021049 & 100.00 \\
\hline
\end{tabular}


${ }^{1} \mathrm{H}$ NMR, ${ }^{13} \mathrm{C}$ NMR and HPLC of 5a

(300 MHz, $\mathrm{CDCl}_{3},{ }^{1} \mathrm{H}$ NMR)

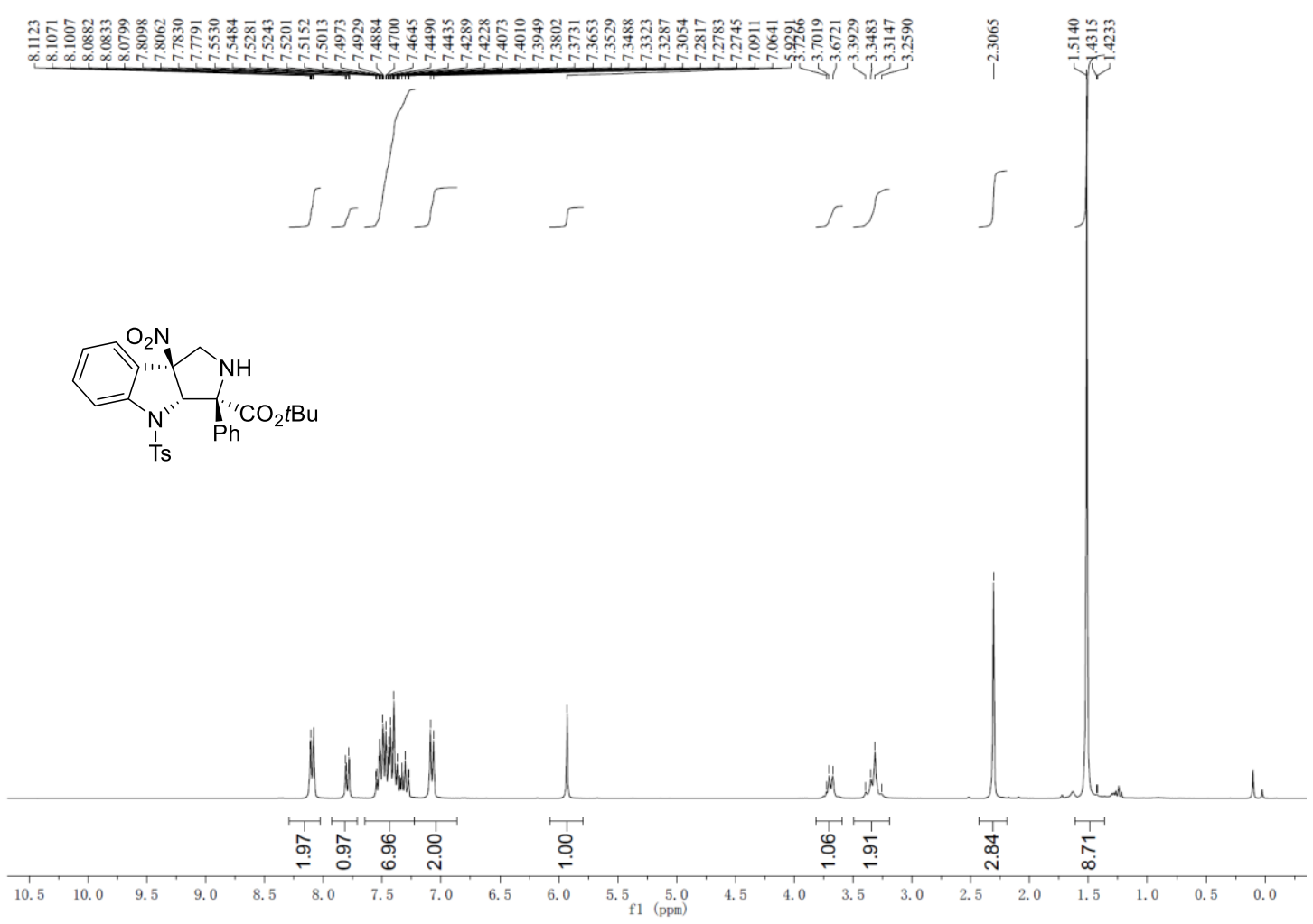

(75 MHz, $\mathrm{CDCl}_{3},{ }^{13} \mathrm{C}$ NMR)
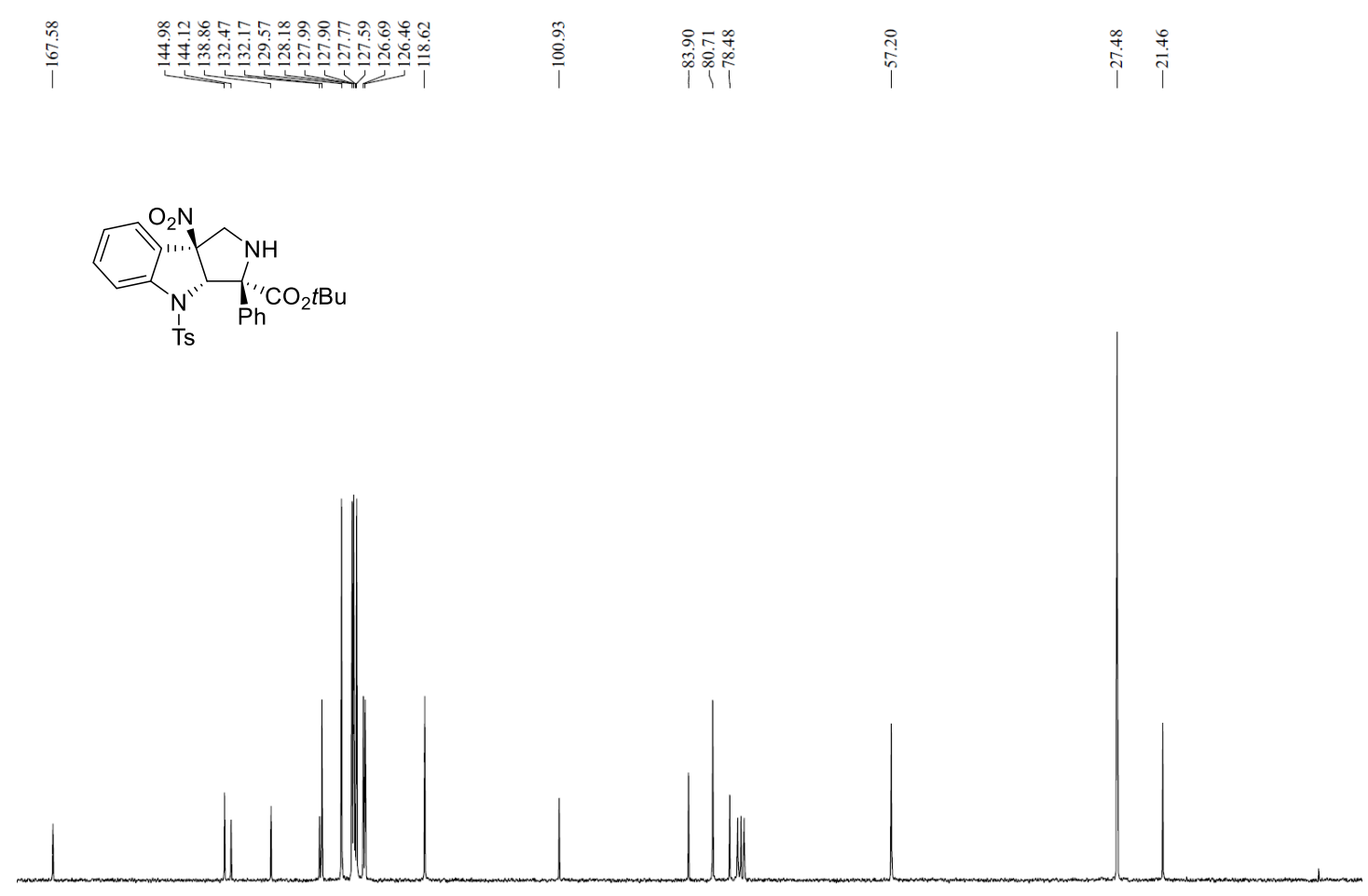

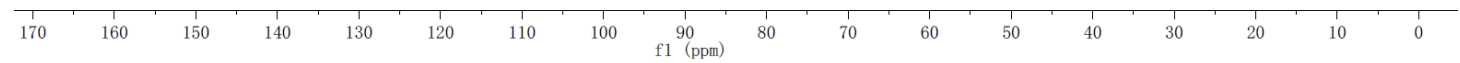




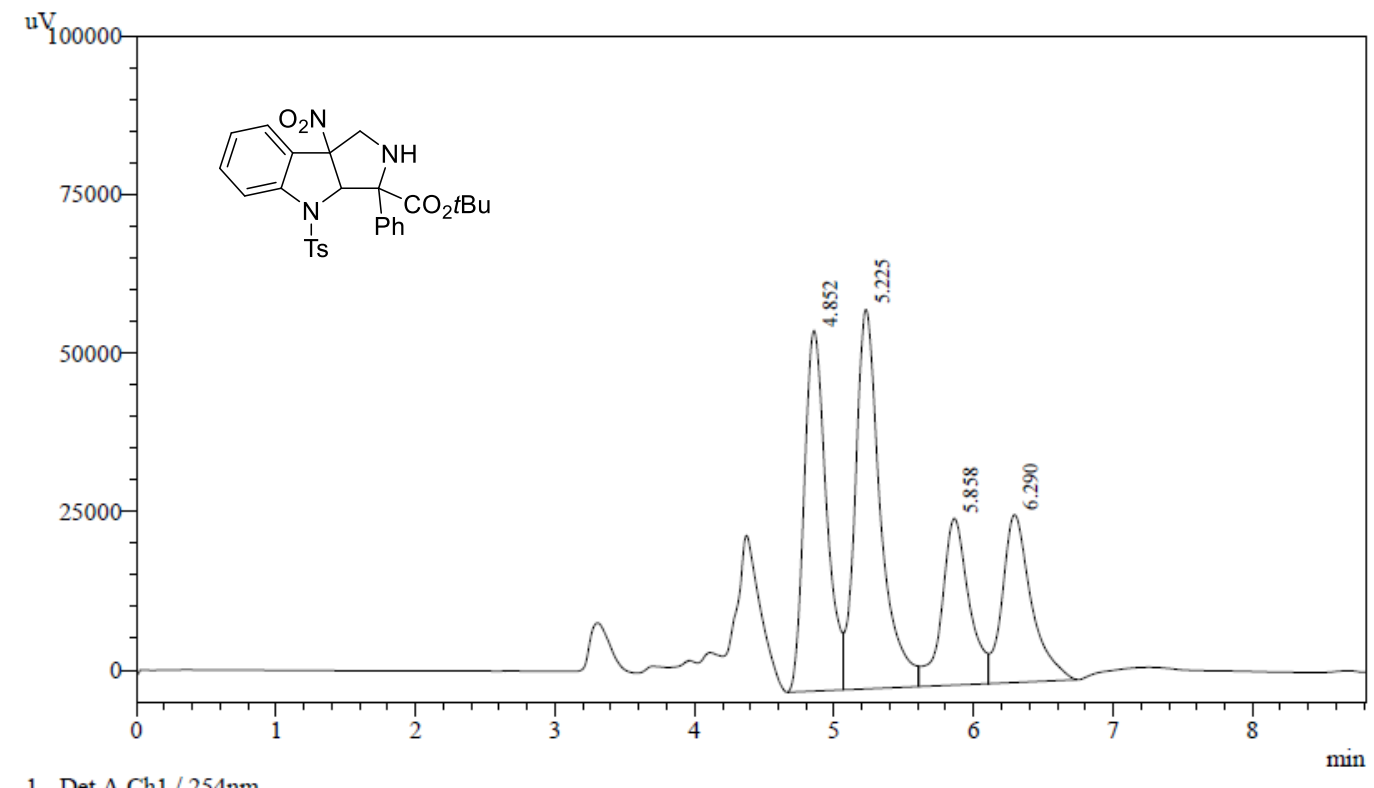

1 Det.A Ch1 / 254nm

Detector A Ch1 254nm

\begin{tabular}{|r|r|r|r|r|r|}
\hline \multicolumn{1}{|c|}{ Peak\# } & Ret. Time & \multicolumn{1}{c|}{ Area } & \multicolumn{1}{c|}{ Height } & \multicolumn{1}{c|}{ Area \% } & Height \% \\
\hline 1 & 4.852 & 630898 & 56902 & 29.713 & 33.581 \\
\hline 2 & 5.225 & 751545 & 59793 & 35.395 & 35.287 \\
\hline 3 & 5.858 & 358043 & 26283 & 16.862 & 15.511 \\
\hline 4 & 6.290 & 382823 & 26470 & 18.030 & 15.621 \\
\hline Total & & 2123310 & 169448 & 100.000 & 100.000 \\
\hline
\end{tabular}

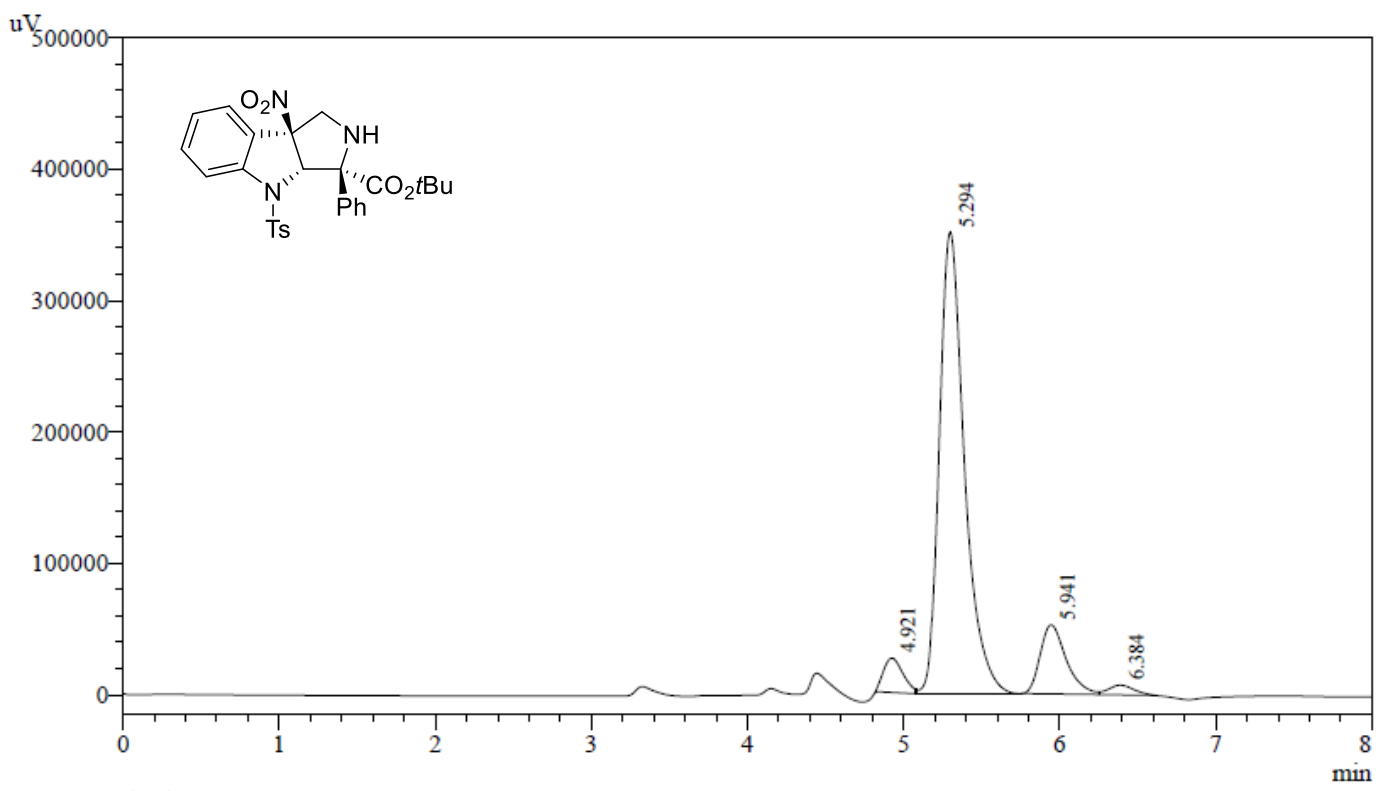

1 Det.A Ch1 / 254nm

Detector A Ch1 254nm

\begin{tabular}{|r|r|r|r|r|r|}
\hline \multicolumn{1}{|c|}{ Peak\# } & \multicolumn{1}{|c|}{ Ret. Time } & \multicolumn{1}{c|}{ Area } & \multicolumn{1}{c|}{ Height } & \multicolumn{1}{c|}{ Area \% } & \multicolumn{1}{c|}{ Height \% } \\
\hline 1 & 4.921 & 227166 & 26141 & 4.688 & 5.973 \\
\hline 2 & 5.294 & 3923527 & 351621 & 80.967 & 80.341 \\
\hline 3 & 5.941 & 610077 & 52481 & 12.590 & 11.991 \\
\hline 4 & 6.384 & 85089 & 7419 & 1.756 & 1.695 \\
\hline Total & & 4845860 & 437663 & 100.000 & 100.000 \\
\hline
\end{tabular}


${ }^{1} \mathrm{H}$ NMR, ${ }^{13} \mathrm{C}$ NMR and HPLC of $5 b$

(300 $\mathrm{MHz}, \mathrm{CDCl}_{3},{ }^{1} \mathrm{H}$ NMR)

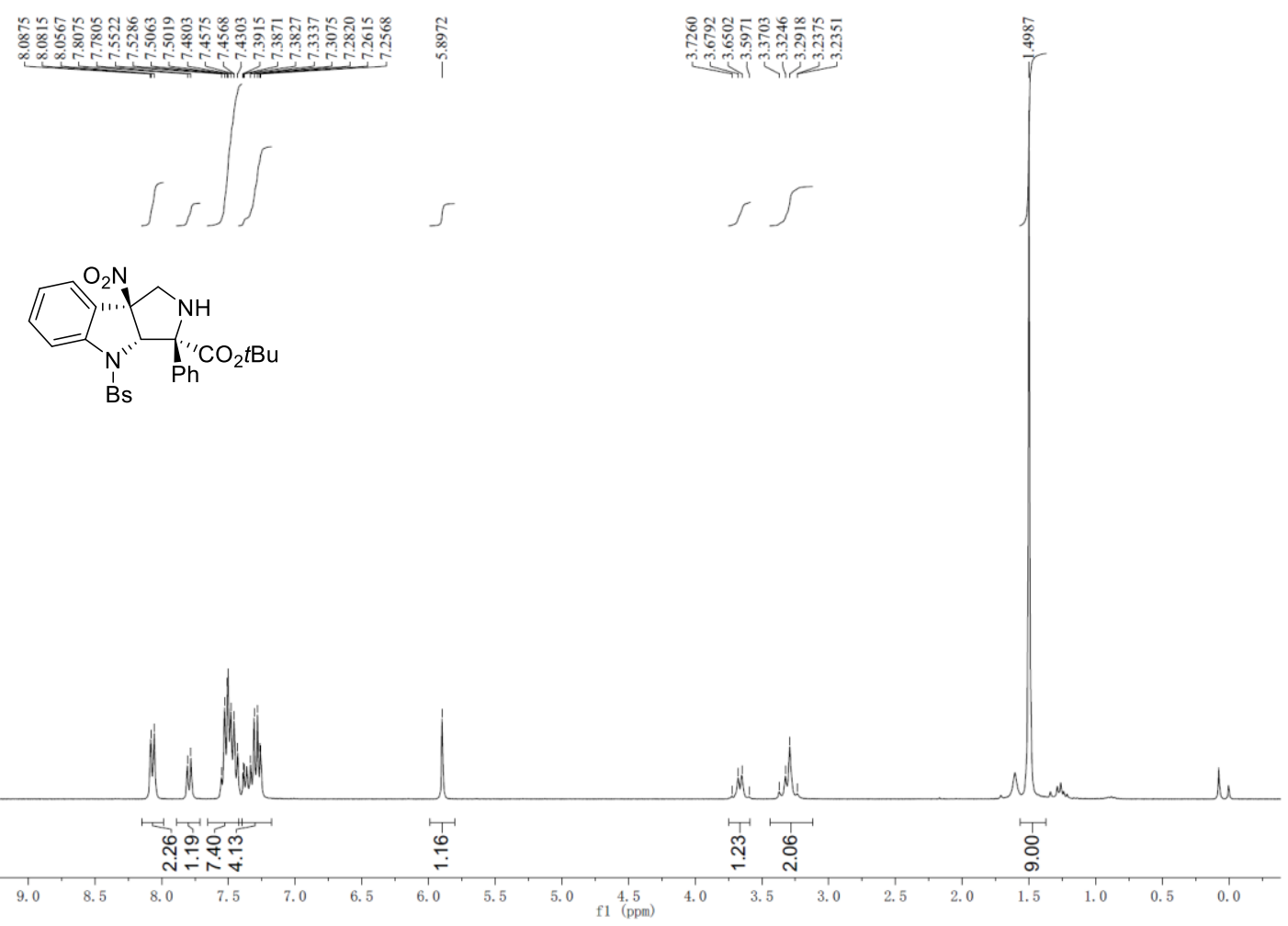

$\left(75 \mathrm{MHz}, \mathrm{CDCl}_{3},{ }^{13} \mathrm{C} \mathrm{NMR}\right)$
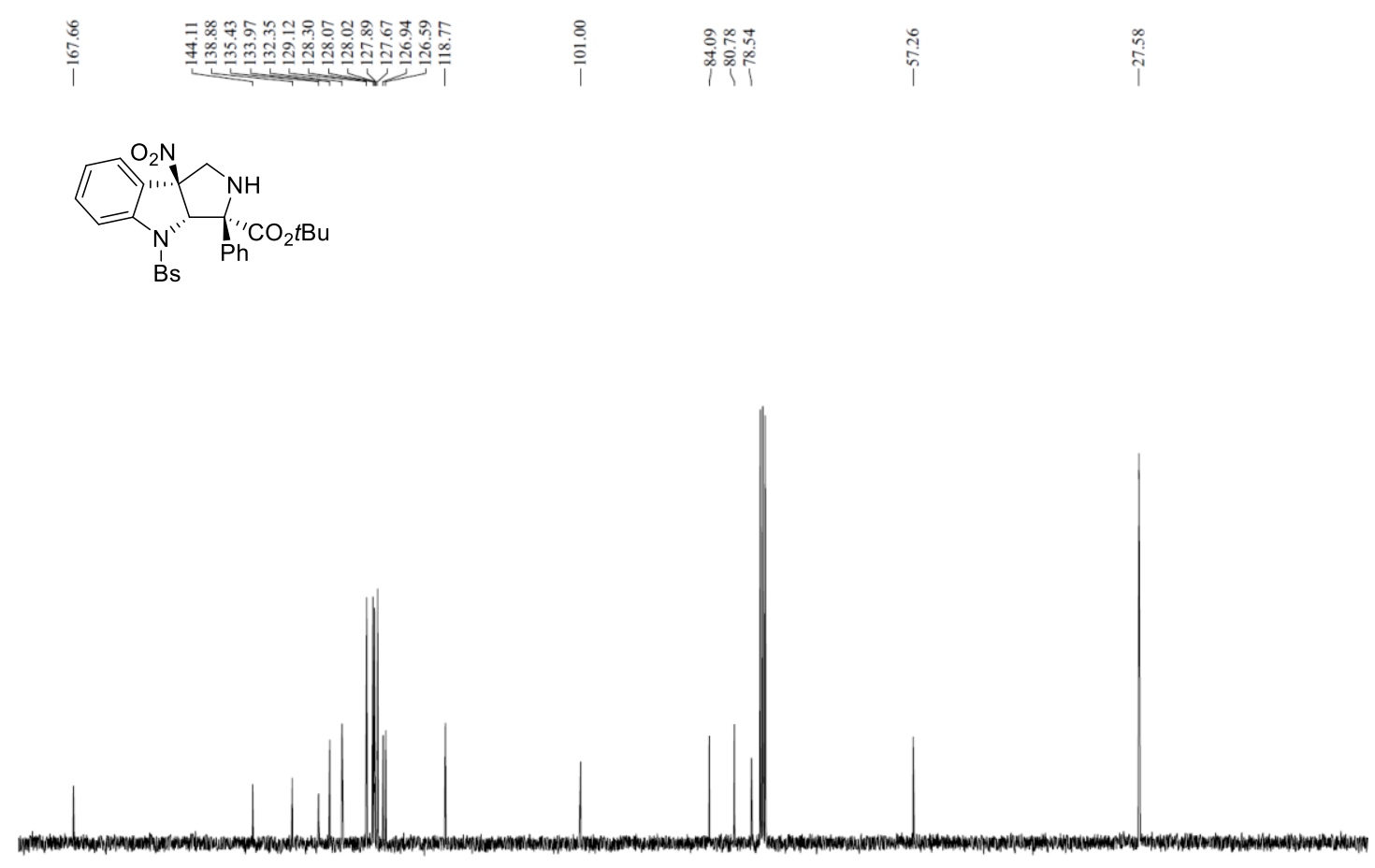


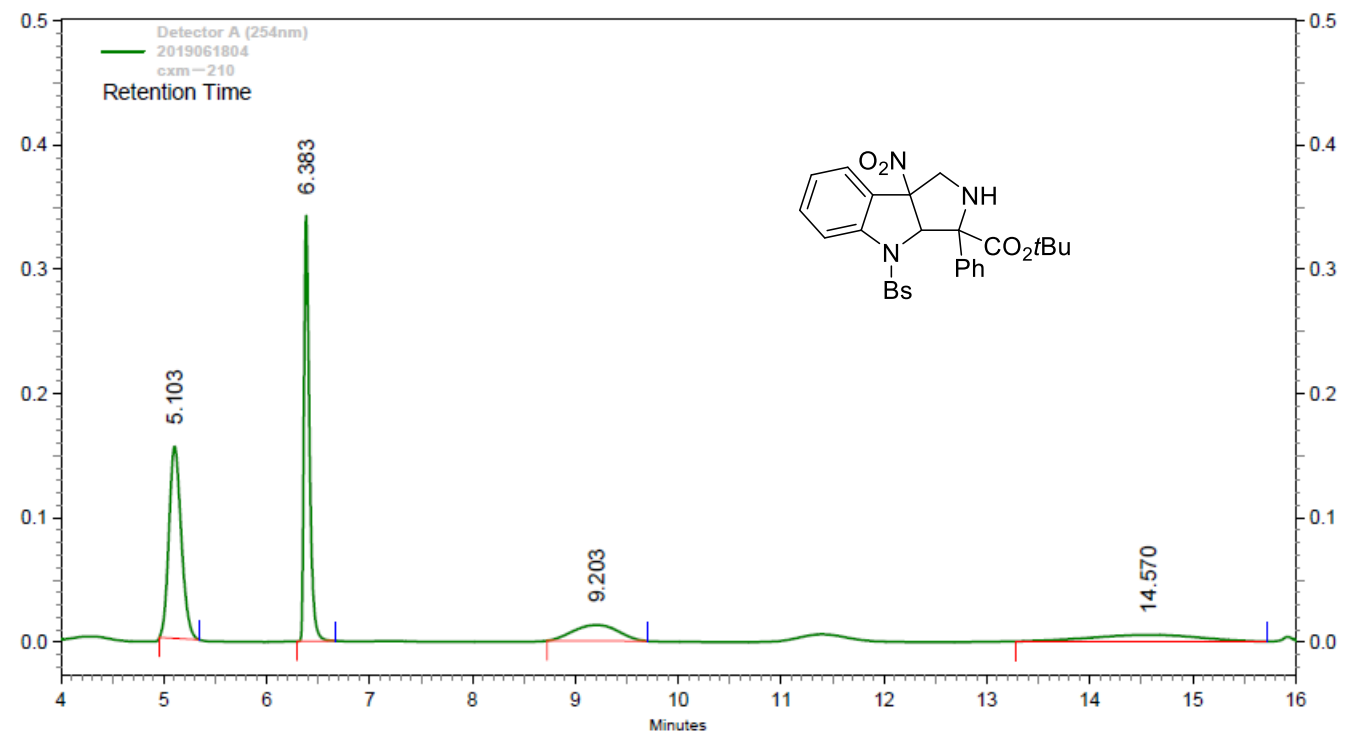

Detector

A (254nm)

Pk \# Retention Time

\begin{tabular}{rrrr} 
Height & $\begin{array}{c}\text { Height } \\
\text { Percent }\end{array}$ & Area & Area Percent \\
\hline 154288 & 29.98 & 1286927 & 38.82 \\
342417 & 66.53 & 1263241 & 38.11 \\
12766 & 2.48 & 388530 & 11.72 \\
5206 & 1.01 & 376043 & 11.34
\end{tabular}

\begin{tabular}{|c|c|c|c|c|}
\hline \multirow[t]{2}{*}{ Totals } & & & & \\
\hline & 514677 & 100.00 & 3314741 & 100.00 \\
\hline
\end{tabular}

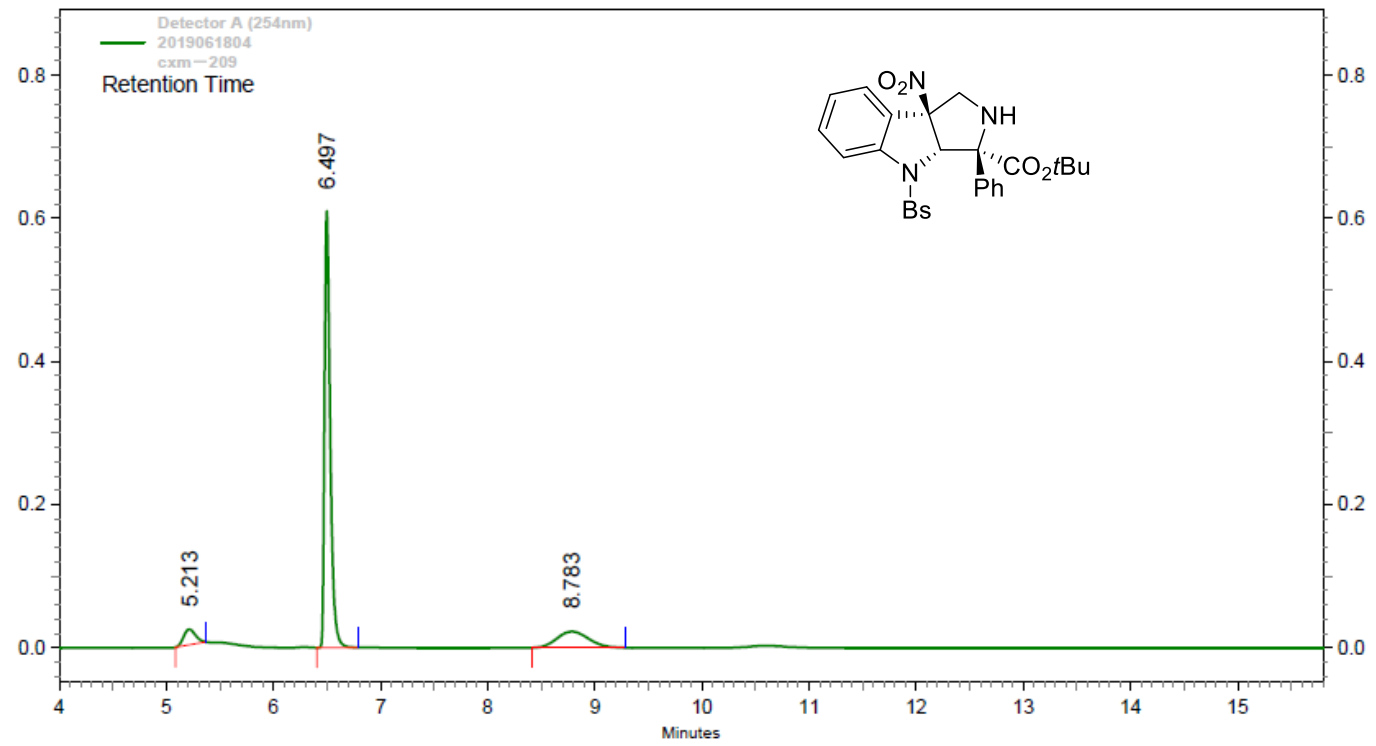

Detector

A (254nm)

Pk \# Retention Time

Height

Height

Area Area Percent

\begin{tabular}{|c|c|c|c|c|c|}
\hline & & & ercent & & \\
\hline 1 & 5.213 & 21750 & 3.32 & 167511 & 5.82 \\
\hline 2 & 6.497 & 610371 & 93.24 & 2246956 & 78.06 \\
\hline 3 & 8.783 & 22500 & 3.44 & 464154 & 16.12 \\
\hline
\end{tabular}

\begin{tabular}{|c|c|c|c|c|}
\hline Totals & & & & \\
\hline & 654621 & 100.00 & 2878621 & 100.00 \\
\hline
\end{tabular}


${ }^{1} \mathrm{H}$ NMR, ${ }^{13} \mathrm{C}$ NMR and HPLC of $5 \mathrm{c}$

(300 $\mathrm{MHz}, \mathrm{CDCl}_{3},{ }^{1} \mathrm{H} \mathrm{NMR}$ )

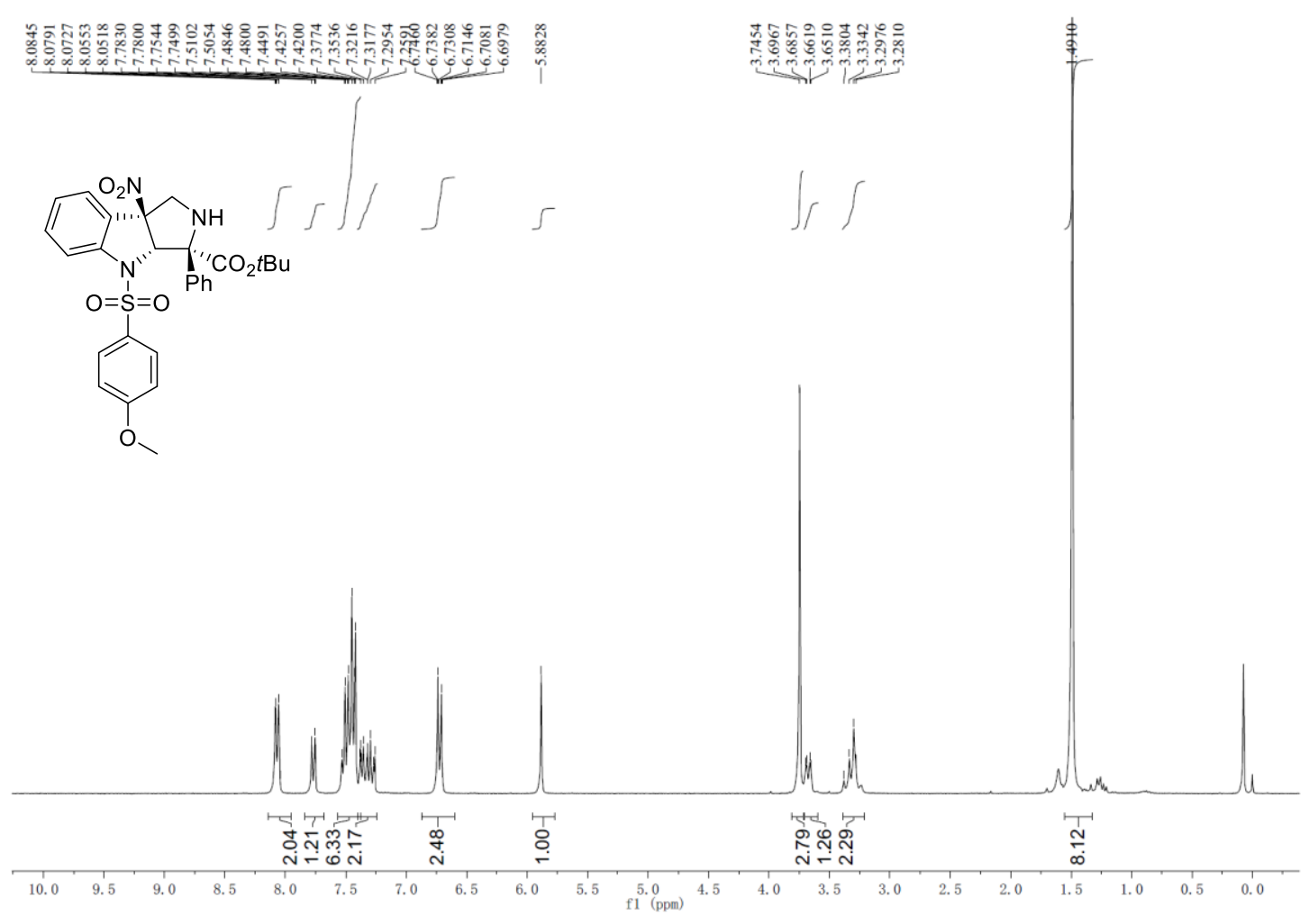

(75 $\left.\mathrm{MHz}, \mathrm{CDCl}_{3},{ }^{13} \mathrm{C} \mathrm{NMR}\right)$

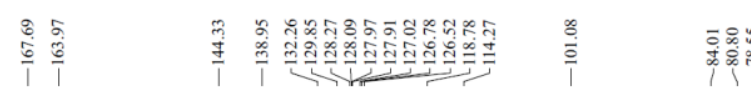
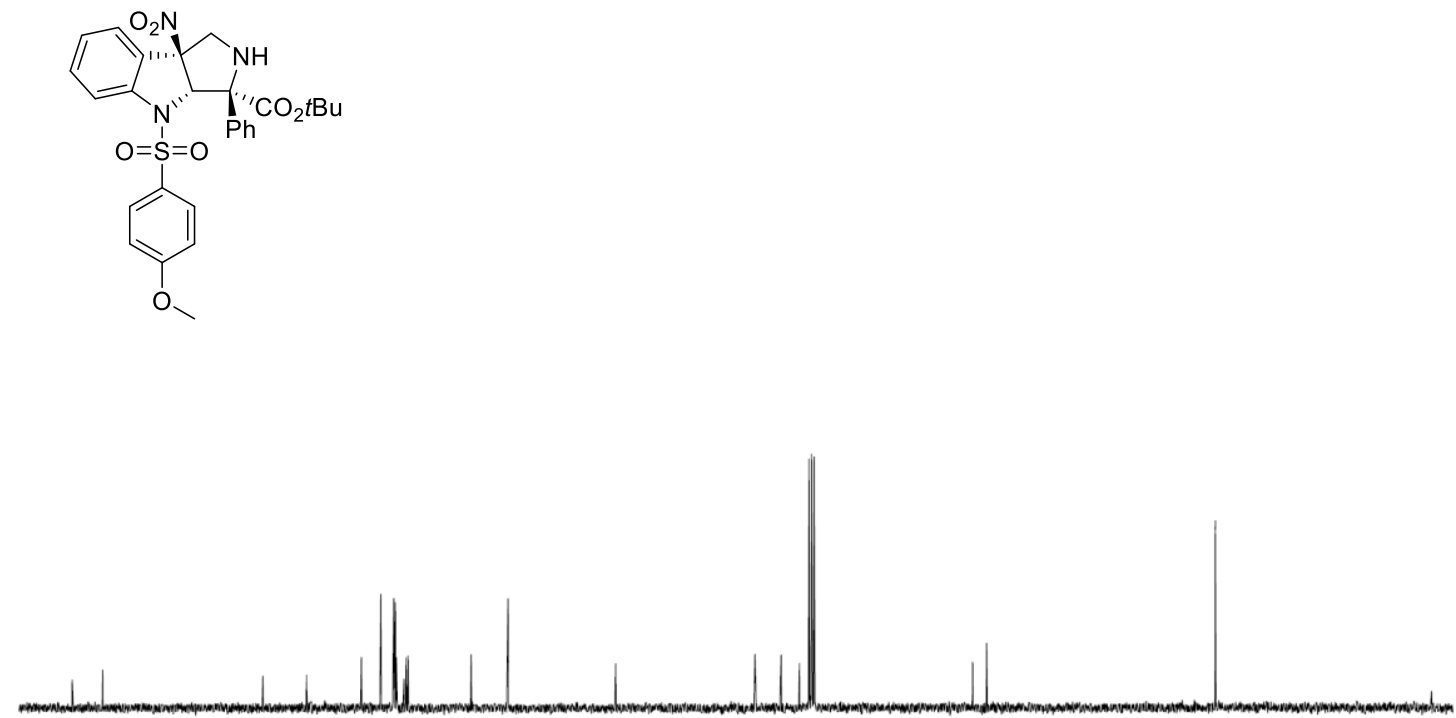

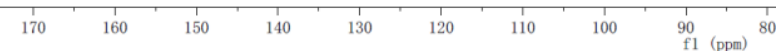




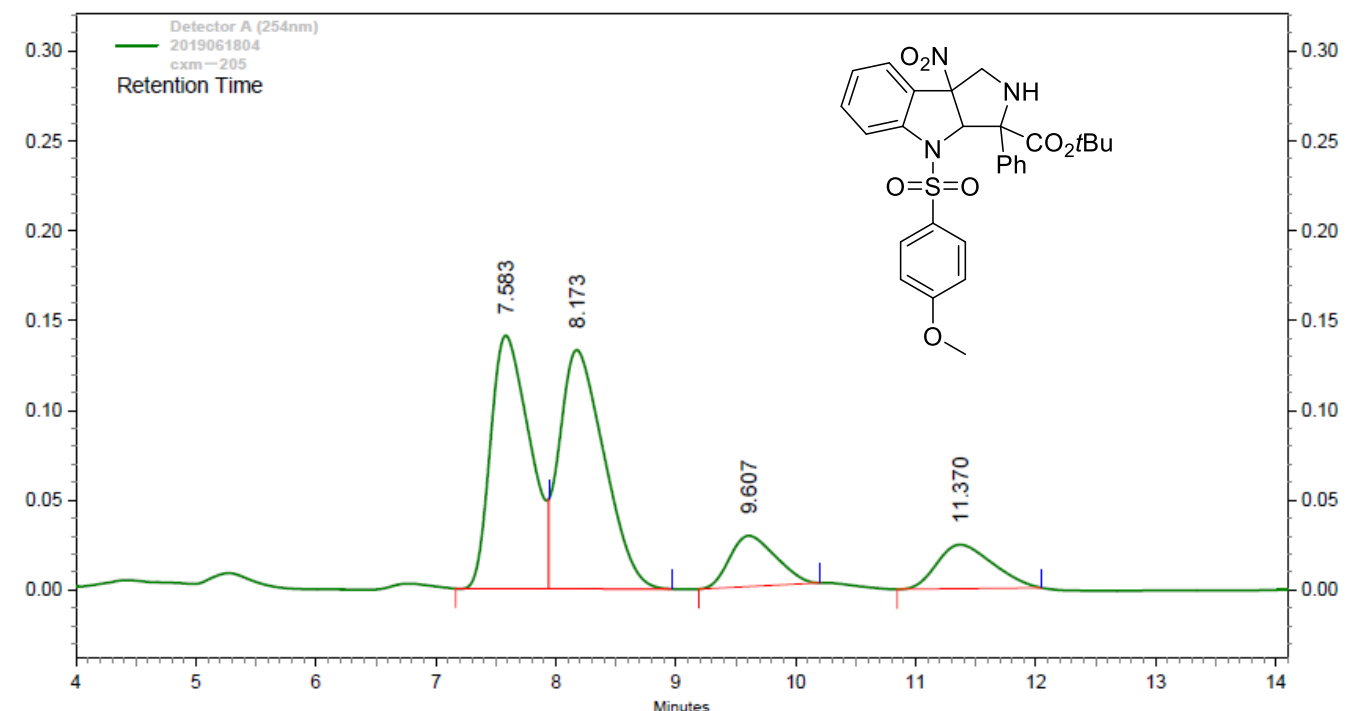

Detector

A $(254 \mathrm{~nm})$

Pk \# Retention Time

Height

Height

Area Area Percent

\begin{tabular}{|c|c|c|c|c|c|}
\hline & & & ercent & & \\
\hline 1 & 7.583 & 140928 & 43.13 & 3210510 & 39.00 \\
\hline 2 & 8.173 & 132992 & 40.71 & 3477838 & 42.25 \\
\hline 3 & 9.607 & 28250 & 8.65 & 748679 & 9.09 \\
\hline 4 & 11.370 & 24548 & 7.51 & 795033 & 9.66 \\
\hline
\end{tabular}

\begin{tabular}{|r|r|rr|}
\hline Totals & 326718 & 100.00 & 8232060 \\
\end{tabular}

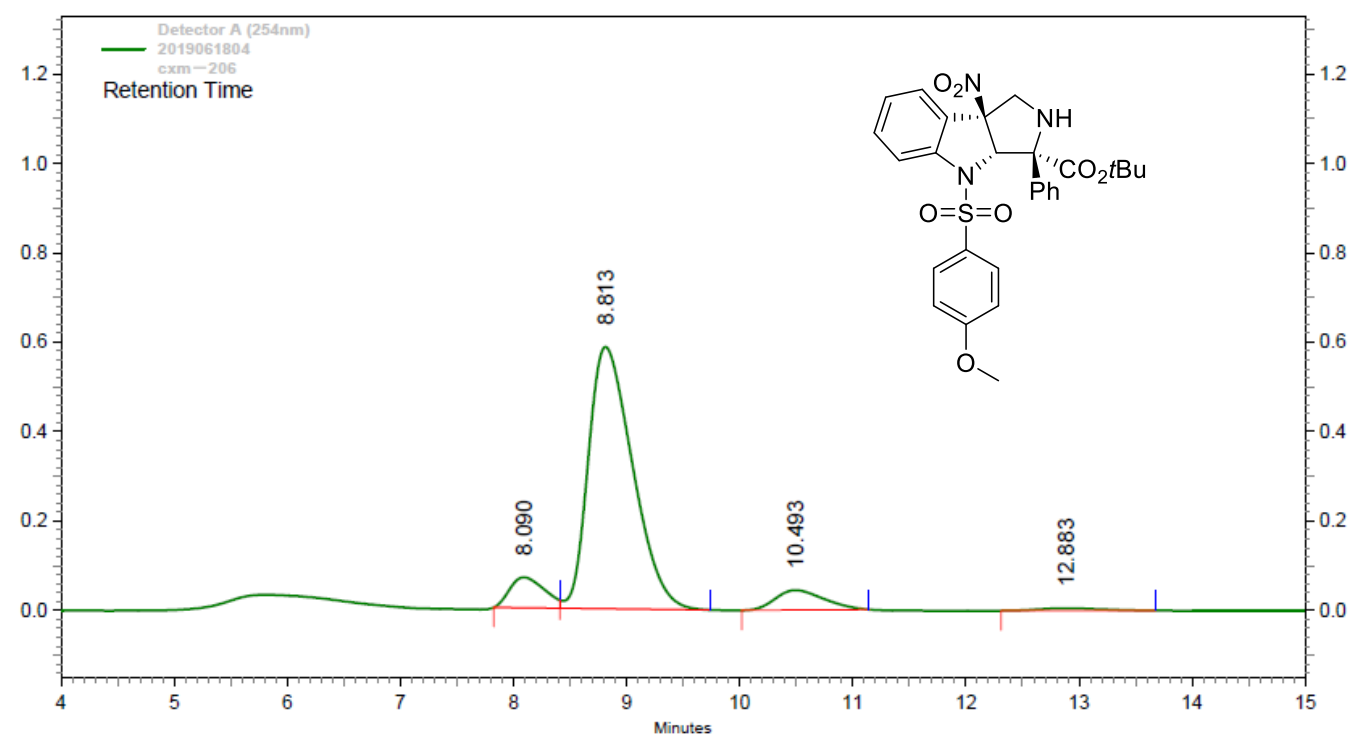

Detector

\begin{tabular}{|c|c|c|c|c|c|}
\hline $\begin{array}{r}\mathrm{A}(254 \mathrm{~nm}) \\
\mathrm{Pk} \#\end{array}$ & Retention Time & Height & $\begin{array}{l}\text { Height } \\
\text { Percent }\end{array}$ & Area & Area Percent \\
\hline 1 & 8.090 & 68128 & 9.69 & 1445033 & 7.64 \\
\hline 2 & 8.813 & 585402 & 83.29 & 15947174 & 84.34 \\
\hline 3 & 10.493 & 44283 & 6.30 & 1317240 & 6.97 \\
\hline 4 & 12.883 & 5020 & 0.71 & 199079 & 1.05 \\
\hline
\end{tabular}

\begin{tabular}{|c|c|c|c|c|}
\hline Totals & & & & \\
\hline & 702833 & 100.00 & 18908526 & 100.00 \\
\hline
\end{tabular}


${ }^{1} \mathrm{H}$ NMR, ${ }^{13} \mathrm{C}$ NMR, ${ }_{19} \mathrm{~F}$ NMR and HPLC of $5 d$ (300 MHz, $\mathrm{CDCl}_{3},{ }^{1} \mathrm{H}$ NMR)

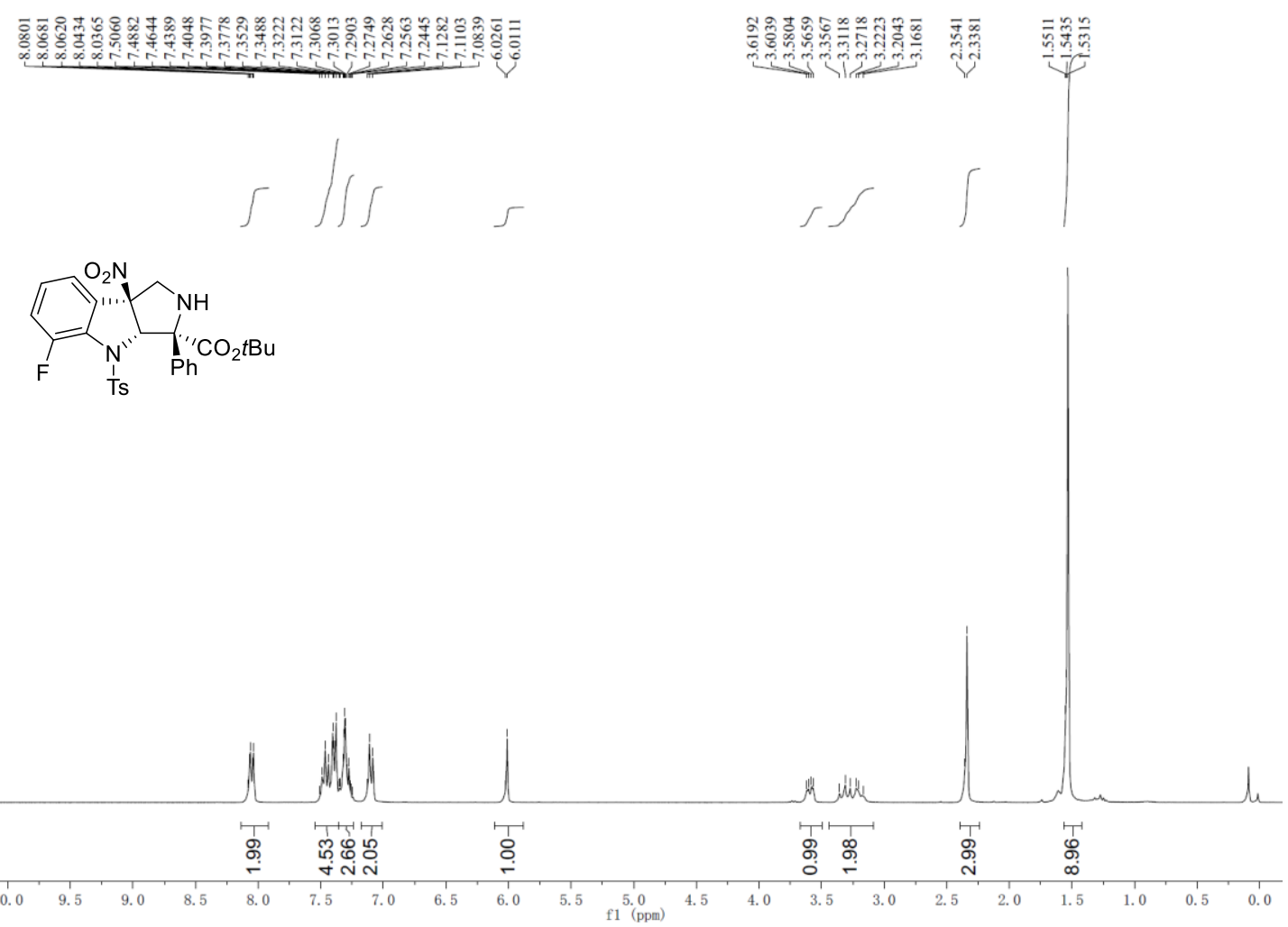

(75 MHz, $\mathrm{CDCl}_{3},{ }^{13} \mathrm{C}$ NMR)
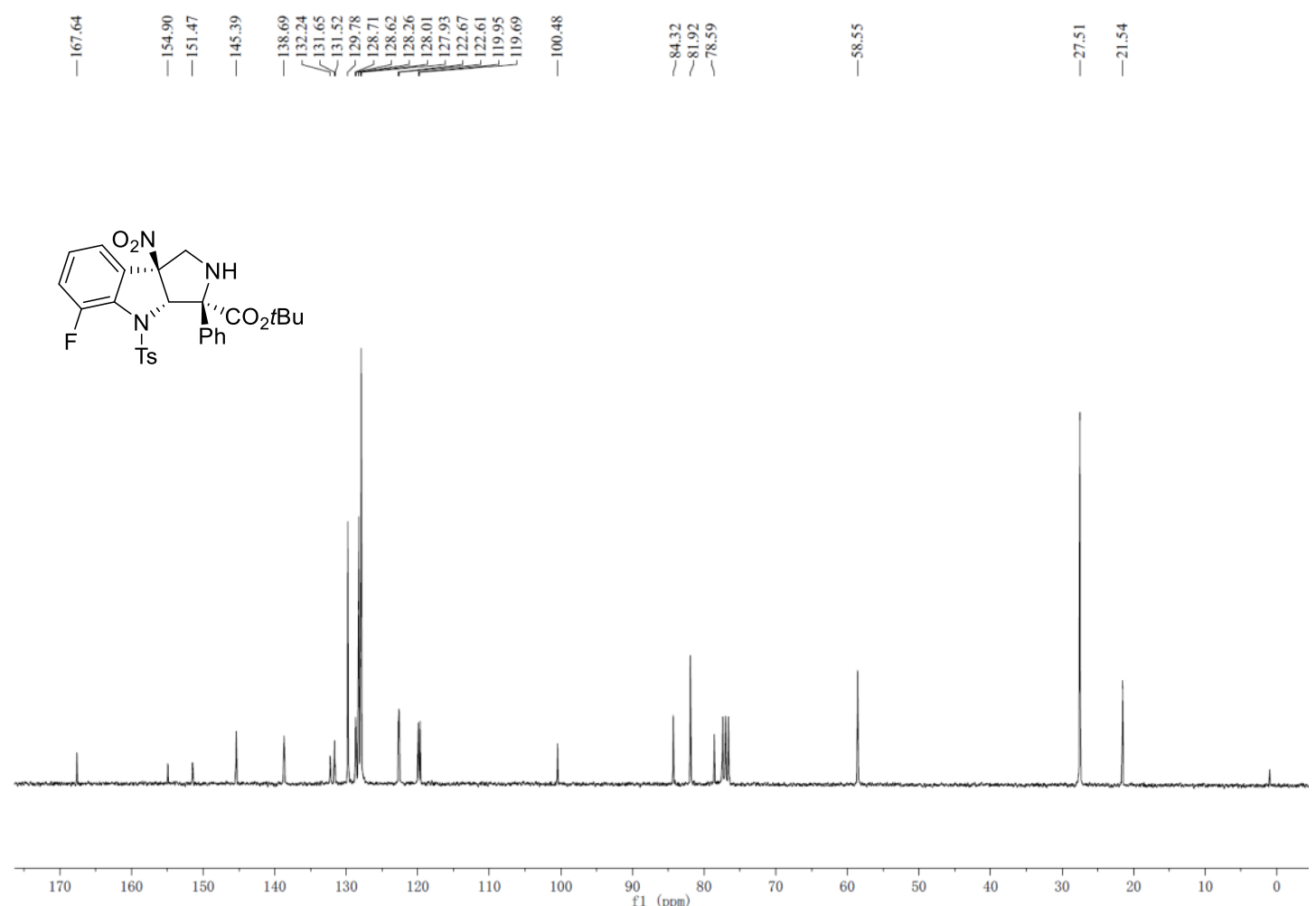
(376 MHz, $\mathrm{CDCl}_{3},{ }^{19} \mathrm{~F}$ NMR)

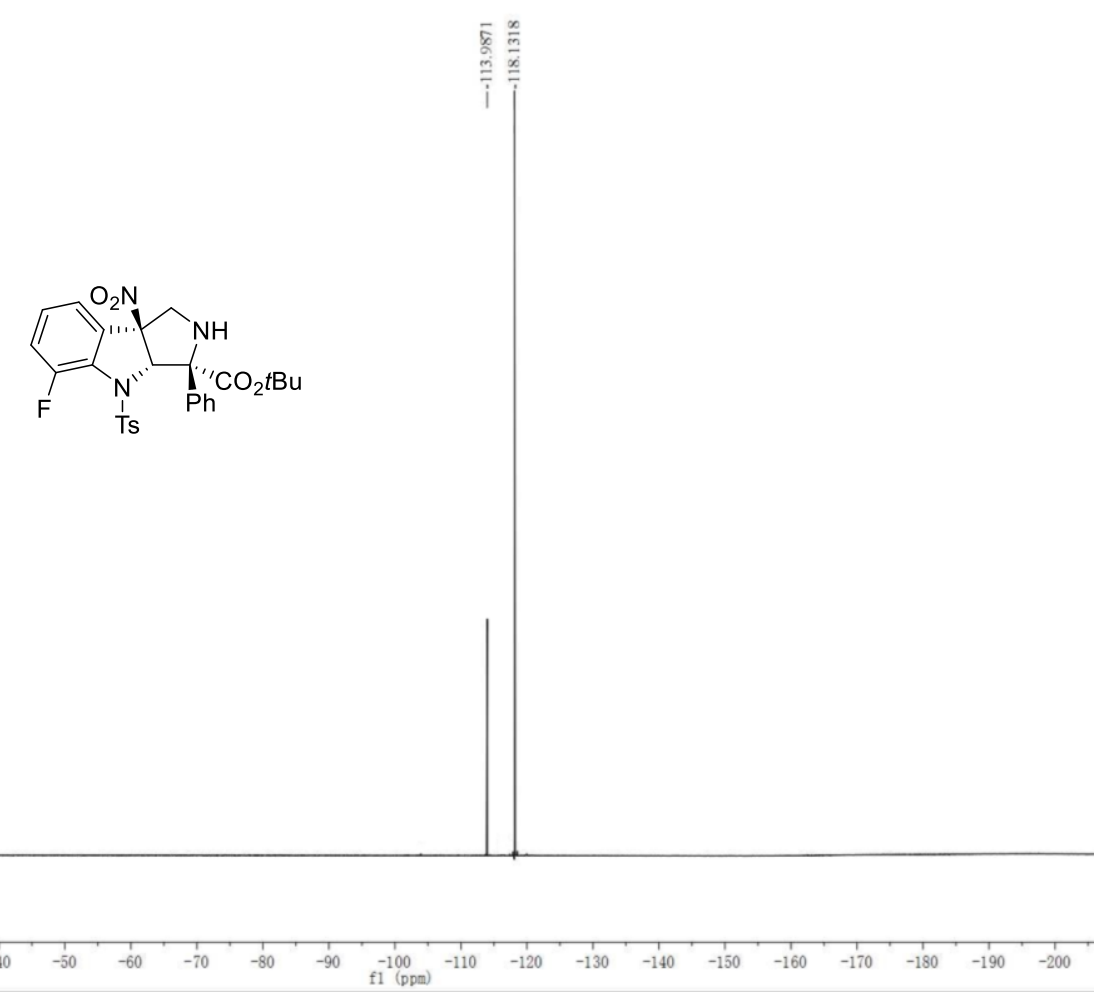




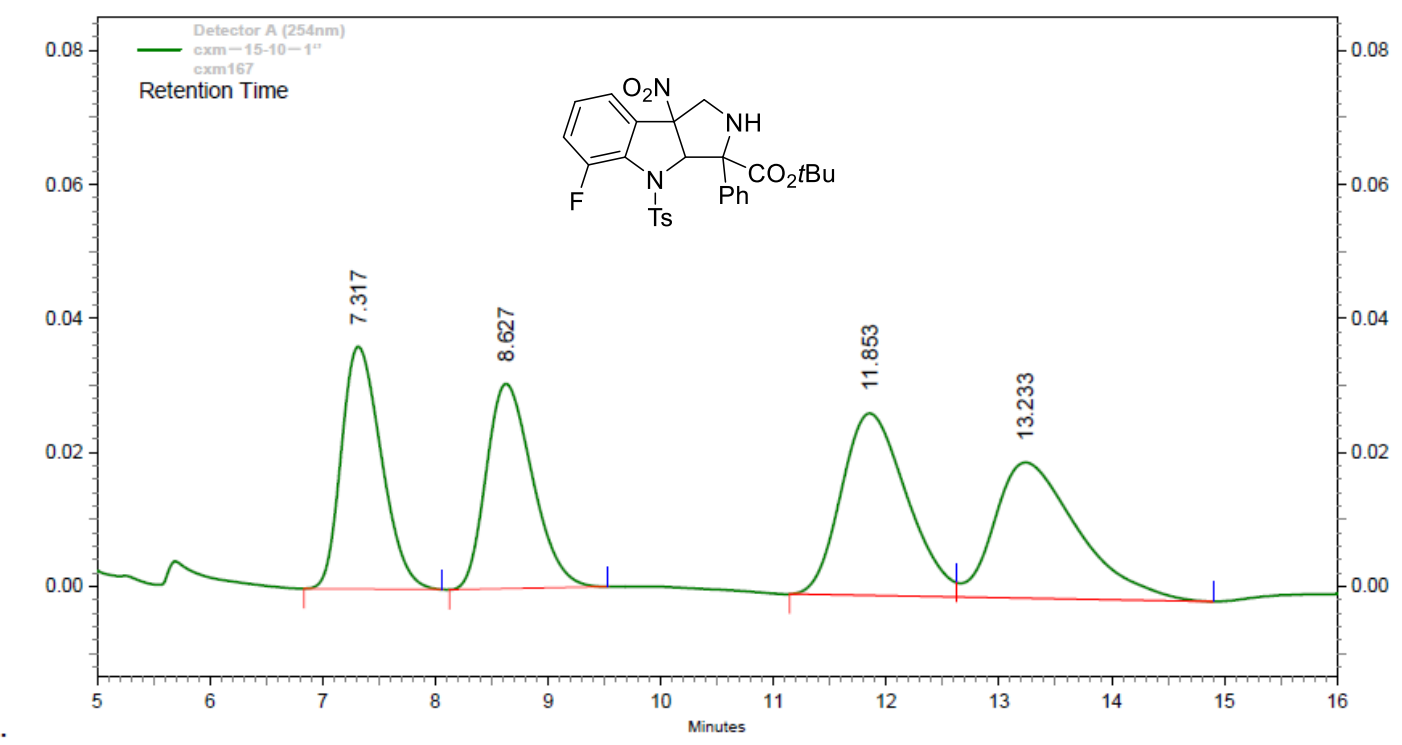

Detector

\begin{tabular}{|c|c|c|c|c|c|}
\hline \multicolumn{6}{|l|}{ A (254nm) } \\
\hline Pk \# & Retention Time & Height & $\begin{array}{l}\text { Height } \\
\text { Percent }\end{array}$ & Area & Area Percent \\
\hline 1 & 7.317 & 36156 & 31.68 & 891150 & 22.84 \\
\hline 2 & 8.627 & 30562 & 26.78 & 871872 & 22.35 \\
\hline 3 & 11.853 & 27164 & 23.80 & 1095718 & 28.09 \\
\hline 4 & 13.233 & 20246 & 17.74 & 1042521 & 26.72 \\
\hline
\end{tabular}

\begin{tabular}{|c|c|c|c|c|}
\hline \multirow{2}{*}{ Totals } & & & & \\
\hline & 114128 & 100.00 & 3901261 & 100.00 \\
\hline
\end{tabular}

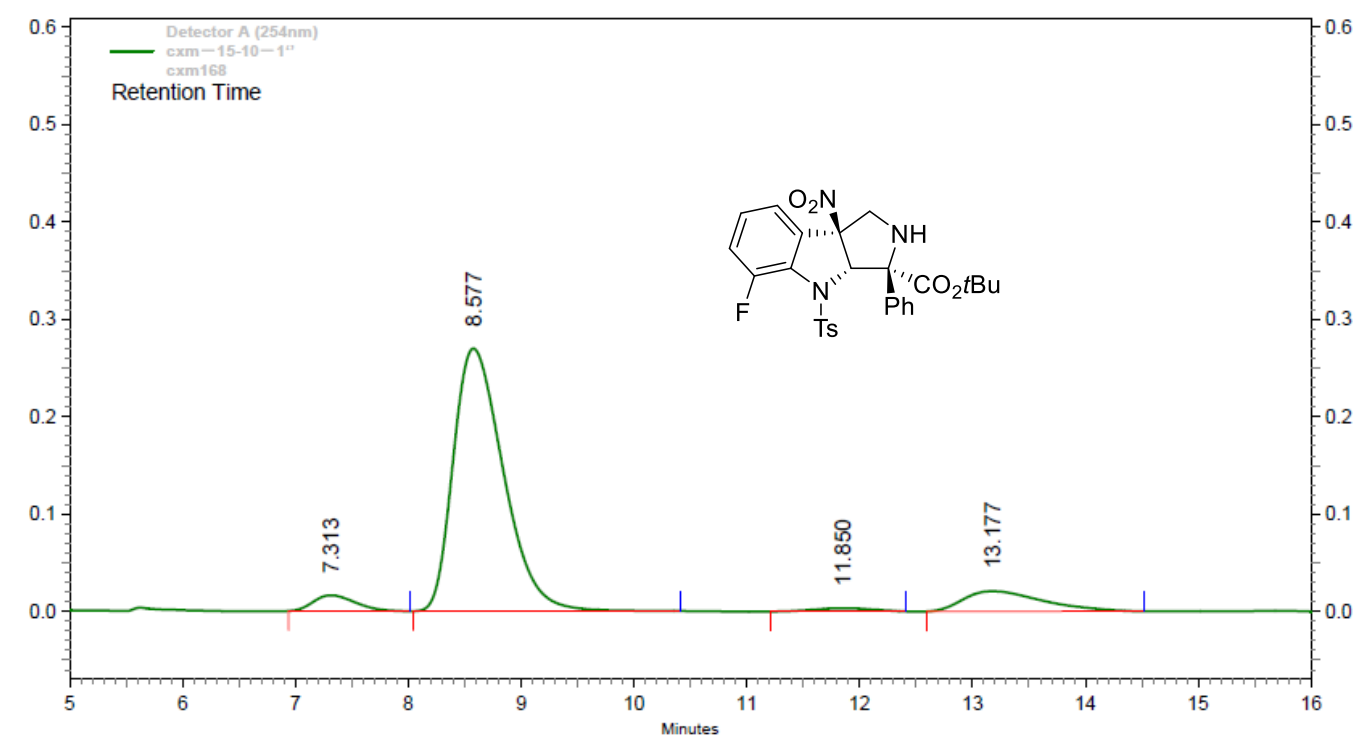

Detector

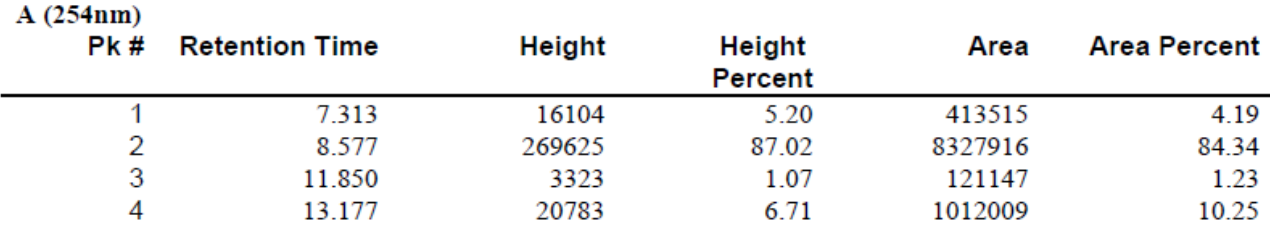

\begin{tabular}{|c|c|c|c|c|}
\hline Totals & & & & \\
\hline & 309835 & 100.00 & 9874587 & 100.00 \\
\hline
\end{tabular}


${ }^{1} \mathrm{H}$ NMR, ${ }^{13} \mathrm{C}$ NMR and HPLC of $5 \mathrm{e}$

(300 $\mathrm{MHz}, \mathrm{CDCl}_{3},{ }^{1} \mathrm{H}$ NMR)
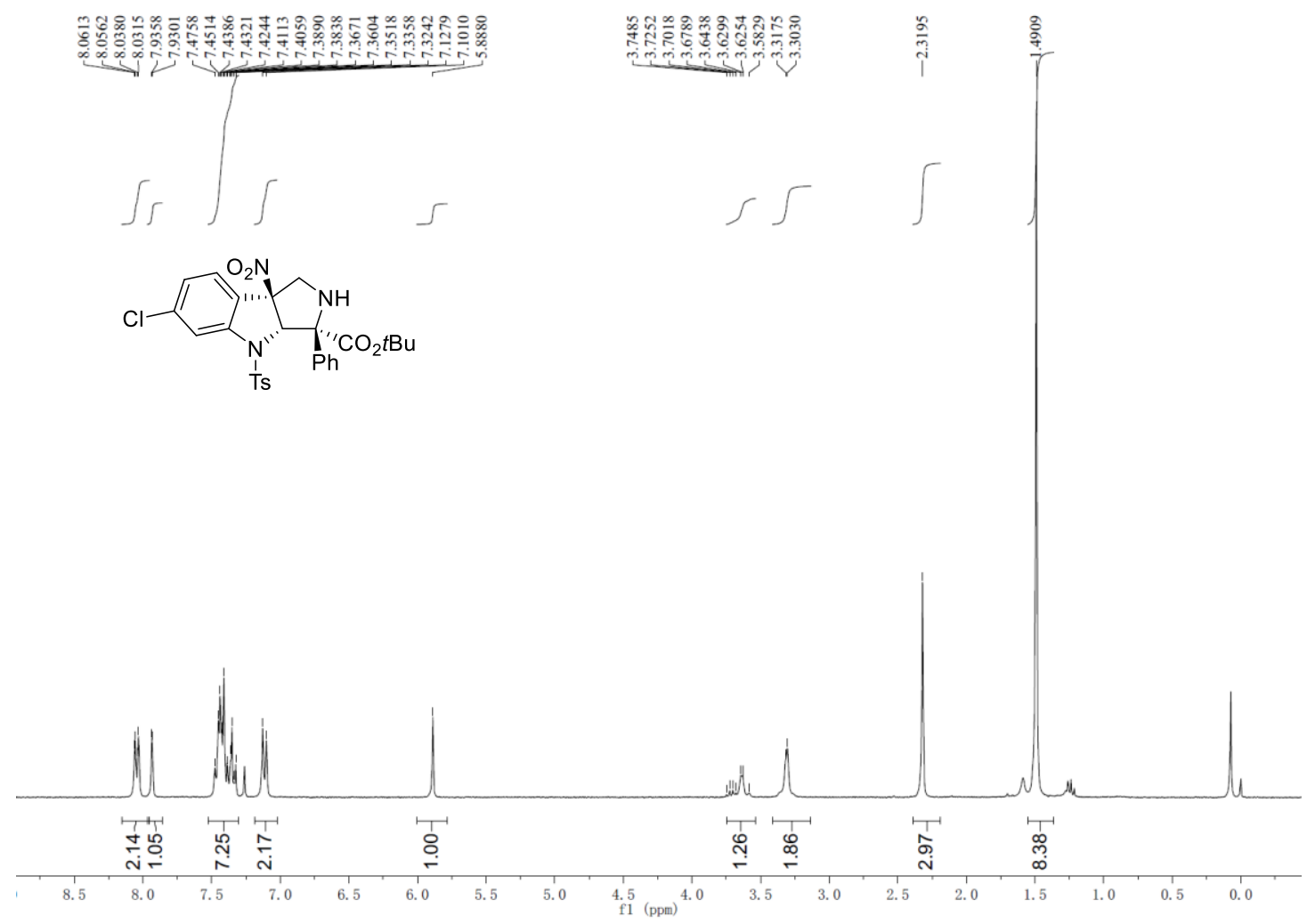

(75 MHz, $\mathrm{CDCl}_{3},{ }^{13} \mathrm{C}$ NMR)
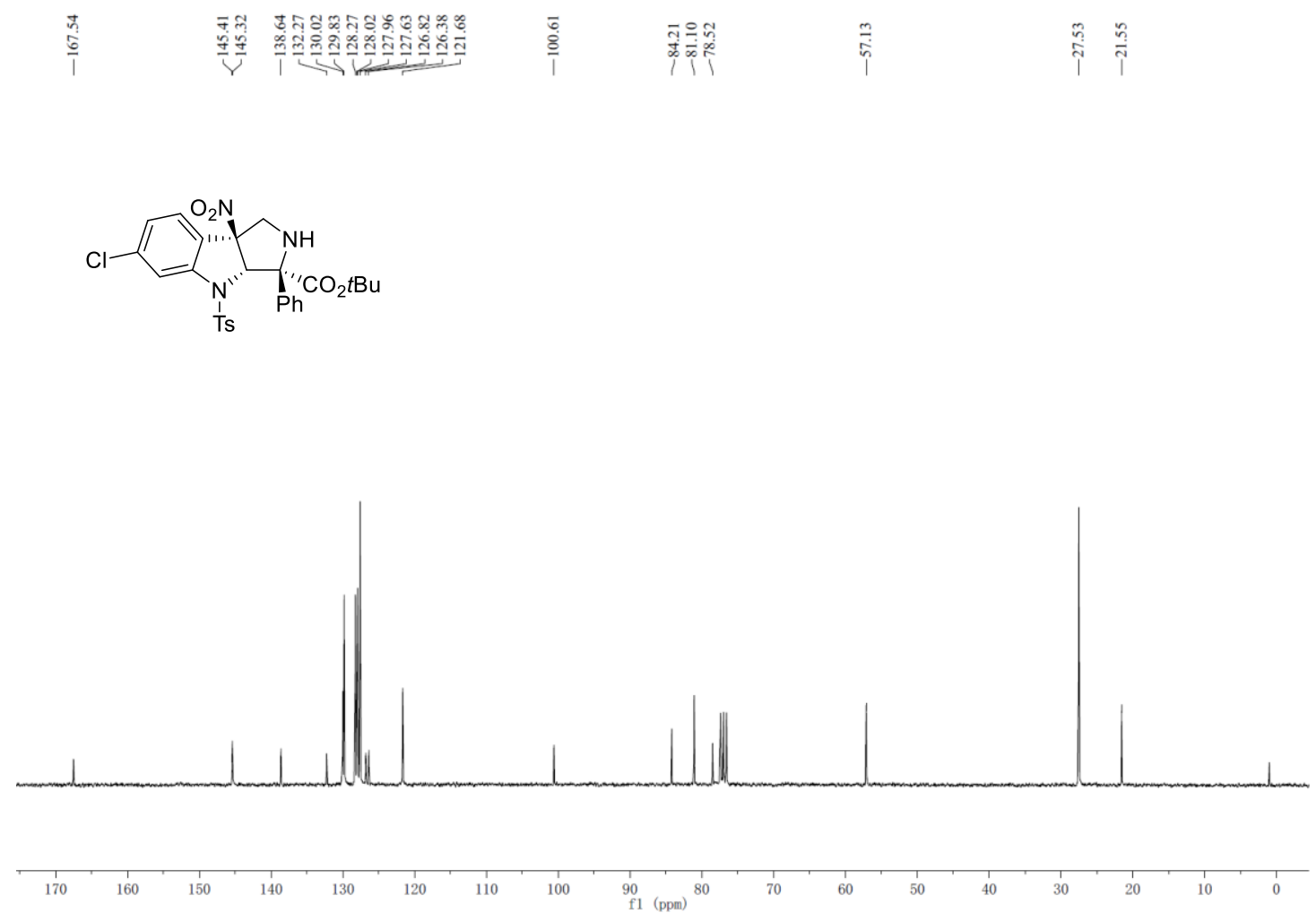


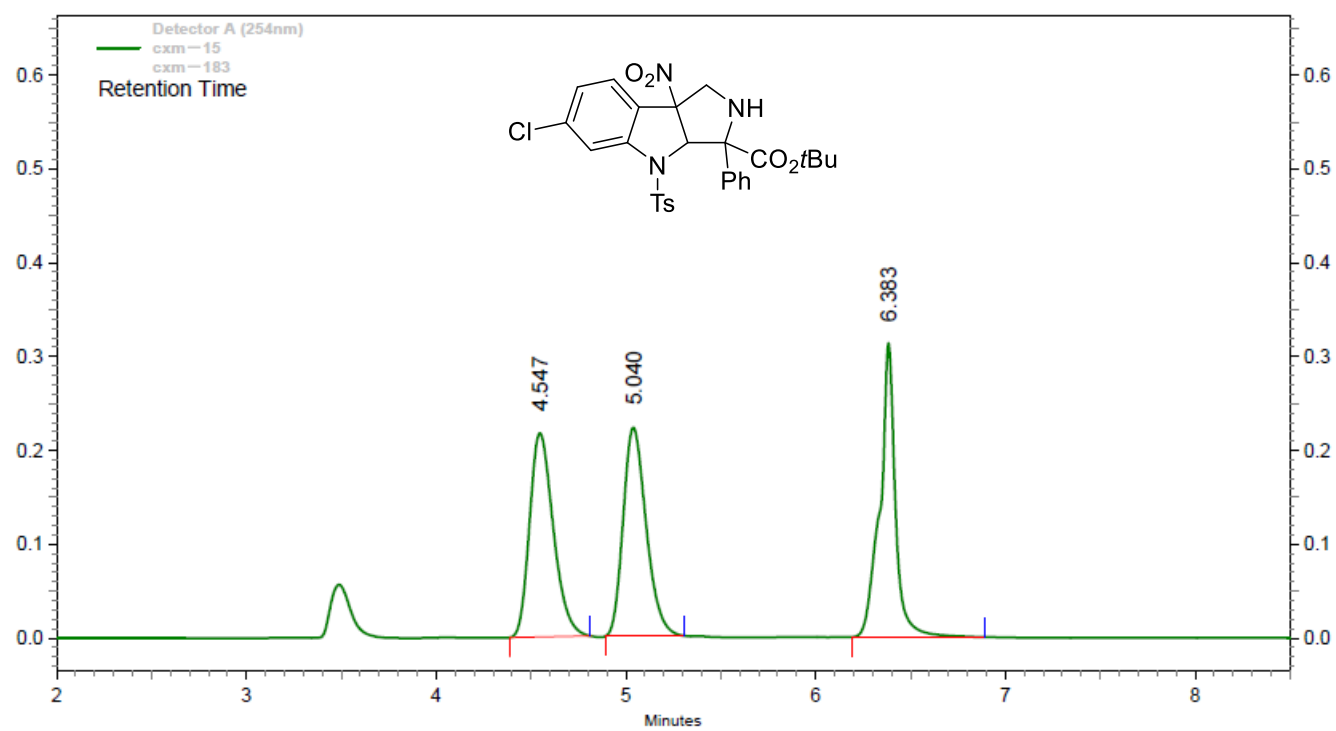

Detector

\begin{tabular}{rrrrrr}
$\begin{array}{r}\text { A (254nm) } \\
\text { Pk \# }\end{array}$ & Retention Time & Height & $\begin{array}{c}\text { Height } \\
\text { Percent }\end{array}$ & Area & Area Percent \\
\hline 1 & 4.547 & 217115 & 28.87 & 1893171 & 34.11 \\
2 & 5.040 & 221793 & 29.50 & 1873297 & 33.75 \\
3 & 6.383 & 313025 & 41.63 & 1783303 & 32.13
\end{tabular}

\begin{tabular}{|c|c|c|c|c|}
\hline \multirow[t]{2}{*}{ Totals } & & & & \\
\hline & 751933 & 100.00 & 5549771 & 100.00 \\
\hline
\end{tabular}

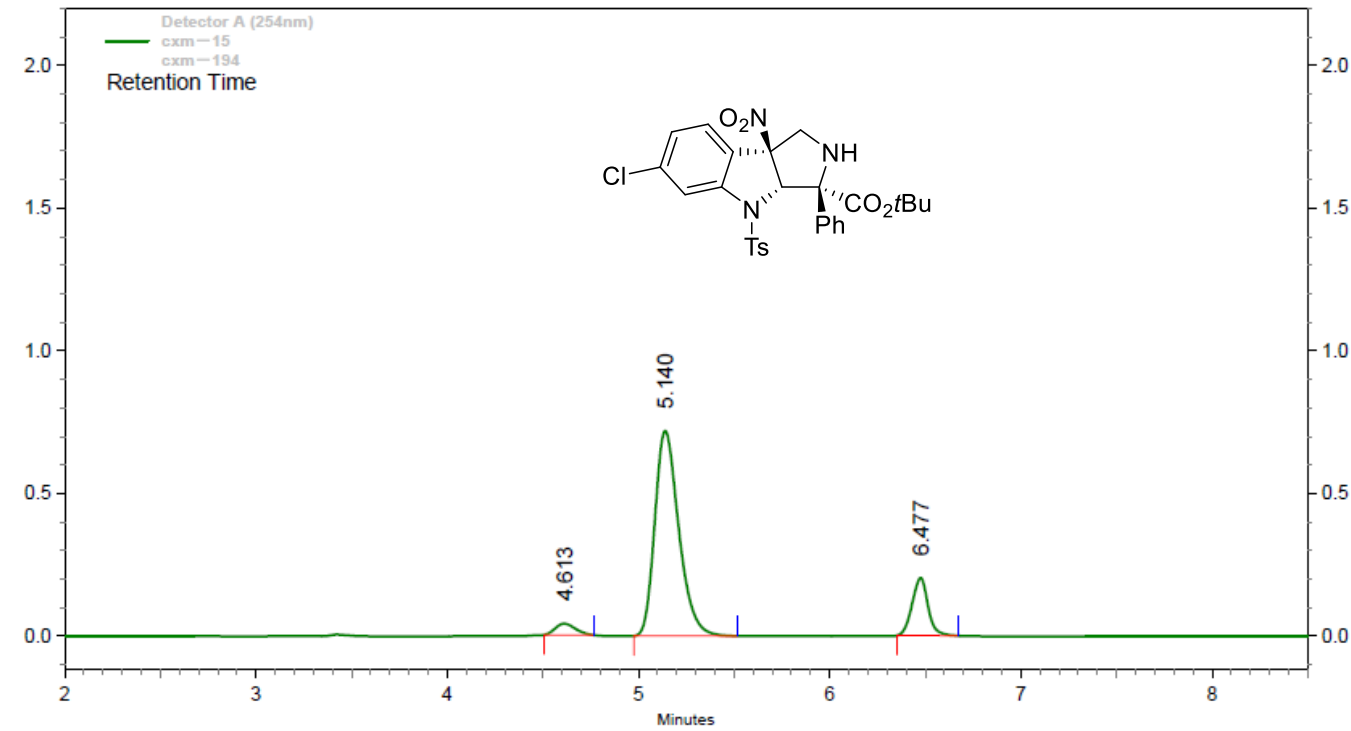

Detector

A (254nm)

Pk \# Retention Time

Height

Height

Area Area Percent

\begin{tabular}{|c|c|c|c|c|c|}
\hline & & & ercent & & \\
\hline 1 & 4.613 & 39629 & 4.12 & 288847 & 3.73 \\
\hline 2 & 5.140 & 719000 & 74.82 & 6283635 & 81.15 \\
\hline 3 & 6.477 & 202329 & 21.05 & 1170641 & 15.12 \\
\hline
\end{tabular}

Totals

960958

100.00

7743123

100.00 
${ }^{1} \mathrm{H}$ NMR, ${ }^{13} \mathrm{C}$ NMR and HPLC of $5 f$

(300 $\mathrm{MHz}, \mathrm{CDCl}_{3},{ }^{1} \mathrm{H}$ NMR)

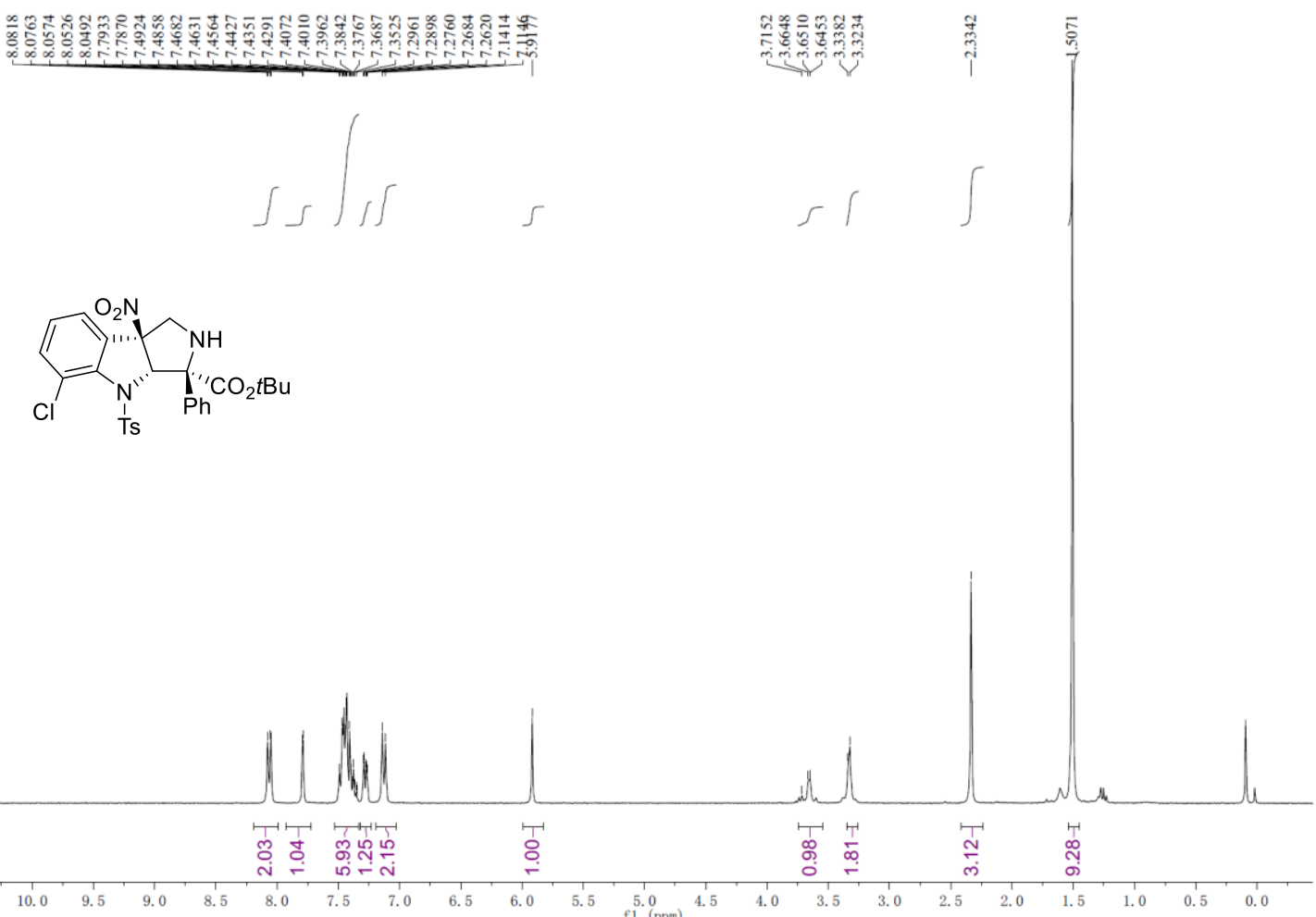

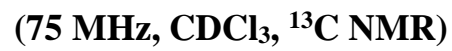

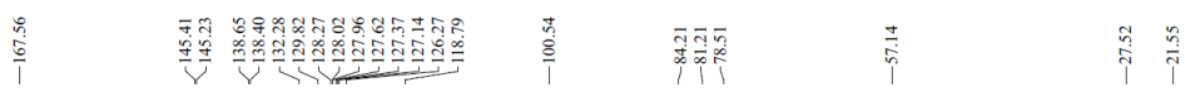
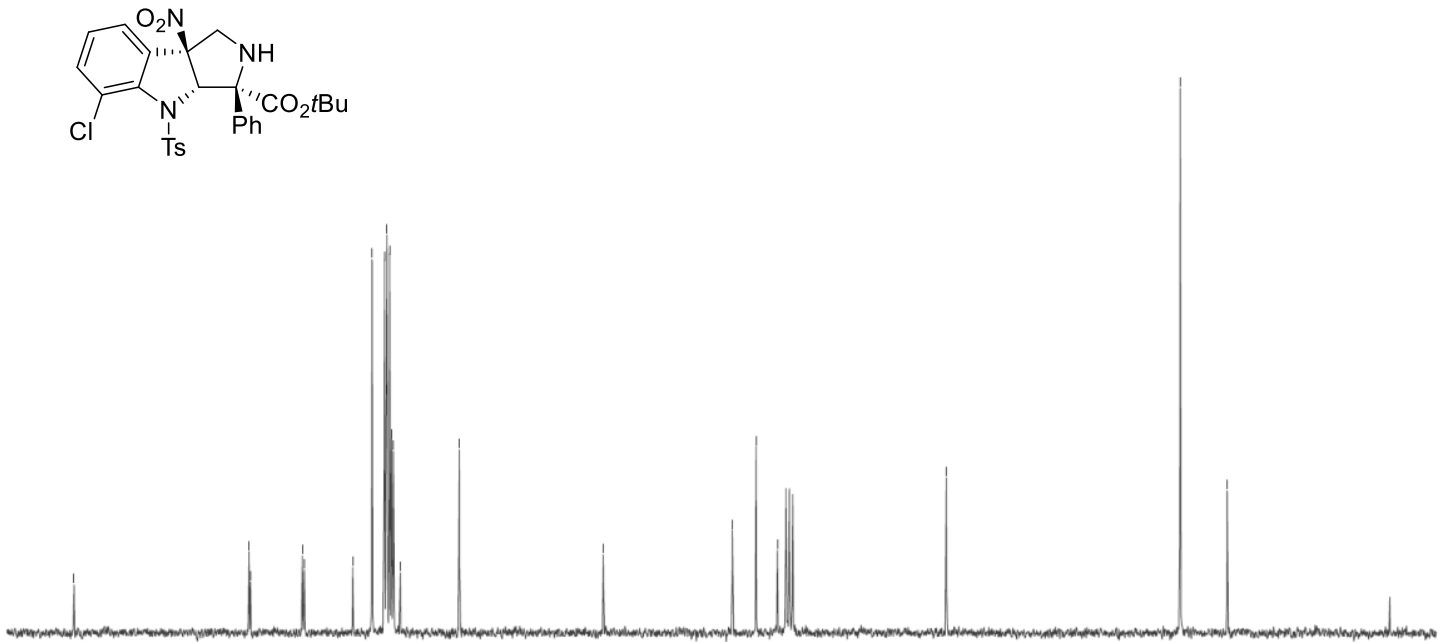

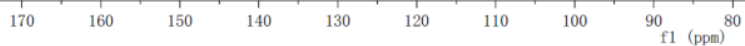




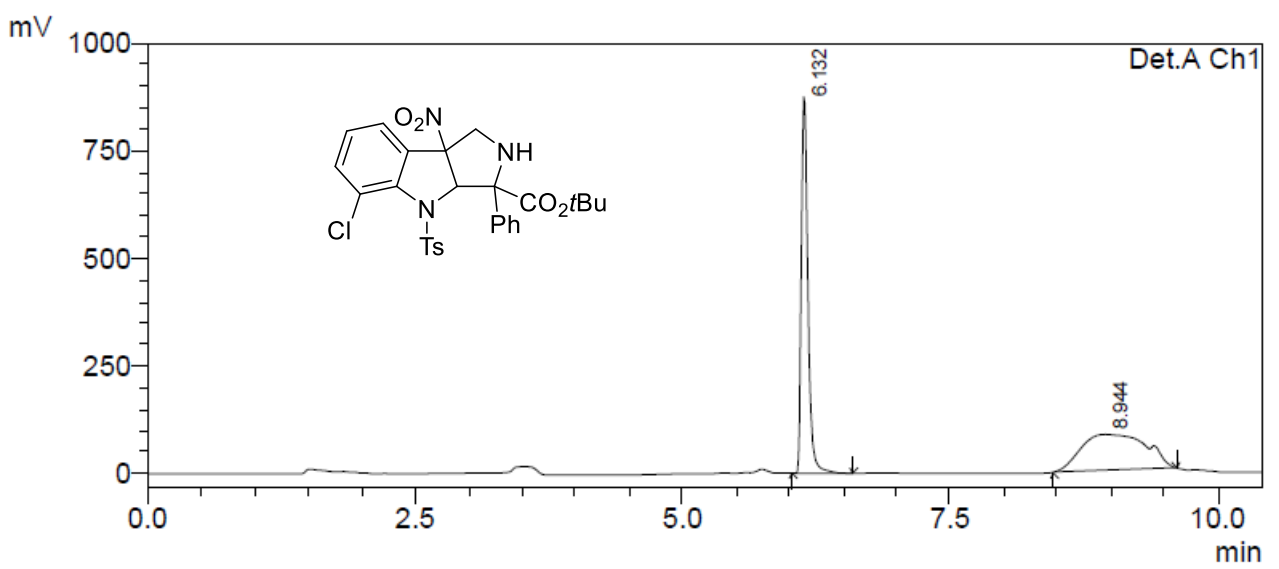

1 Det.A Ch1/254nm

PeakTable
\begin{tabular}{|c|c|c|c|c|} 
Detector A Ch1 254nm \\
\hline Peak\# & Ret. Time & Area & Height & Area \% \\
\hline 1 & 6.132 & 3770889 & 873433 & 51.881 \\
\hline 2 & 8.944 & 3497419 & 82951 & 48.119 \\
\hline Total & & 7268308 & & 100.000 \\
\hline
\end{tabular}

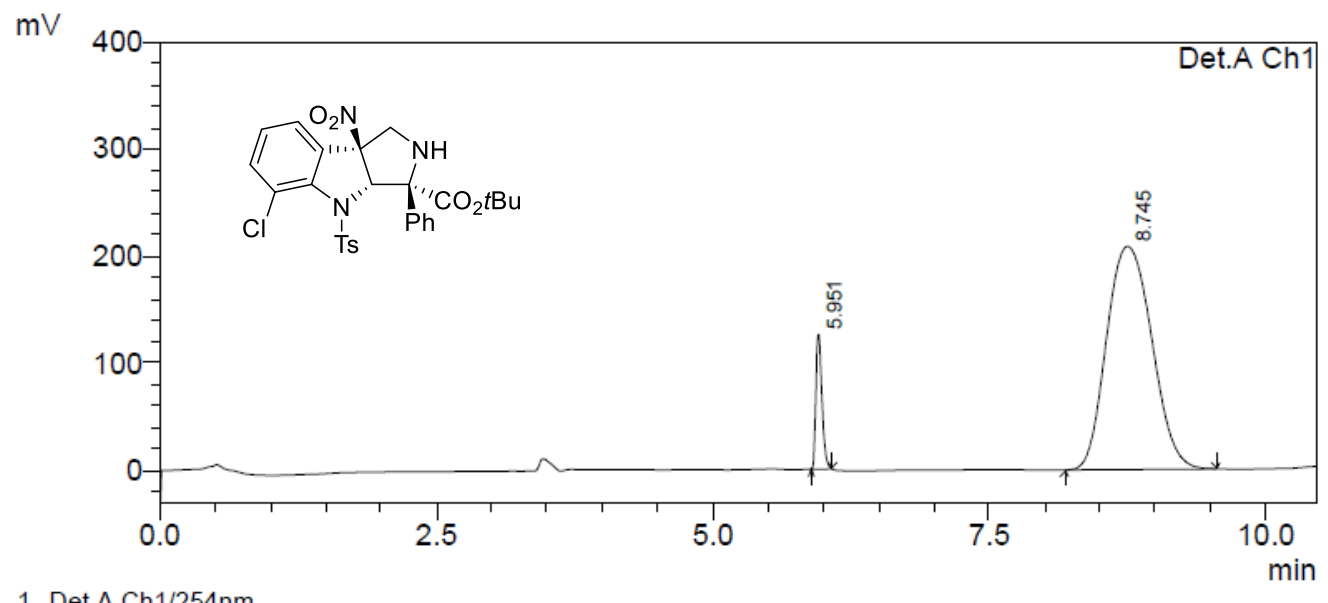

1 Det.A Ch1/254nm

PeakTable

Detector A Ch1 254nm

\begin{tabular}{|c|c|c|c|c|}
\hline Peak\# & Ret. Time & Area & Height & Area \% \\
\hline 1 & 5.951 & 480173 & 125890 & 7.471 \\
\hline 2 & 8.745 & 5946786 & 208182 & 92.529 \\
\hline Total & & 6426959 & & 100.000 \\
\hline
\end{tabular}


${ }^{1} \mathrm{H}$ NMR, ${ }^{13} \mathrm{C}$ NMR and HPLC of $5 \mathrm{~g}$

(300 $\mathrm{MHz}, \mathrm{CDCl}_{3},{ }^{1} \mathrm{H}$ NMR)

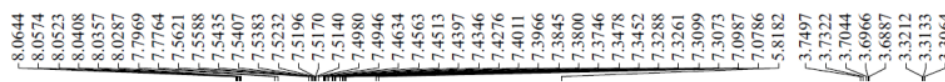

$\left.\int\right\lrcorner / \|_{j} \int$

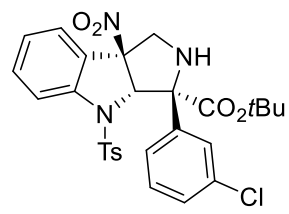

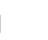

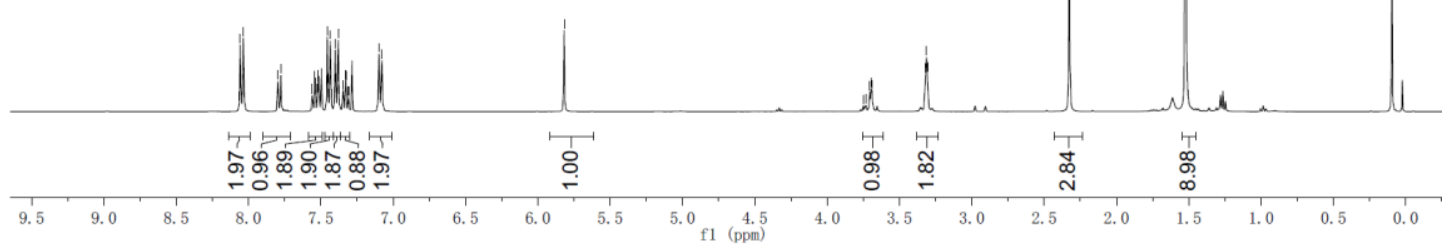

(75 $\mathrm{MHz}, \mathrm{CDCl}_{3},{ }^{13} \mathrm{C}$ NMR)
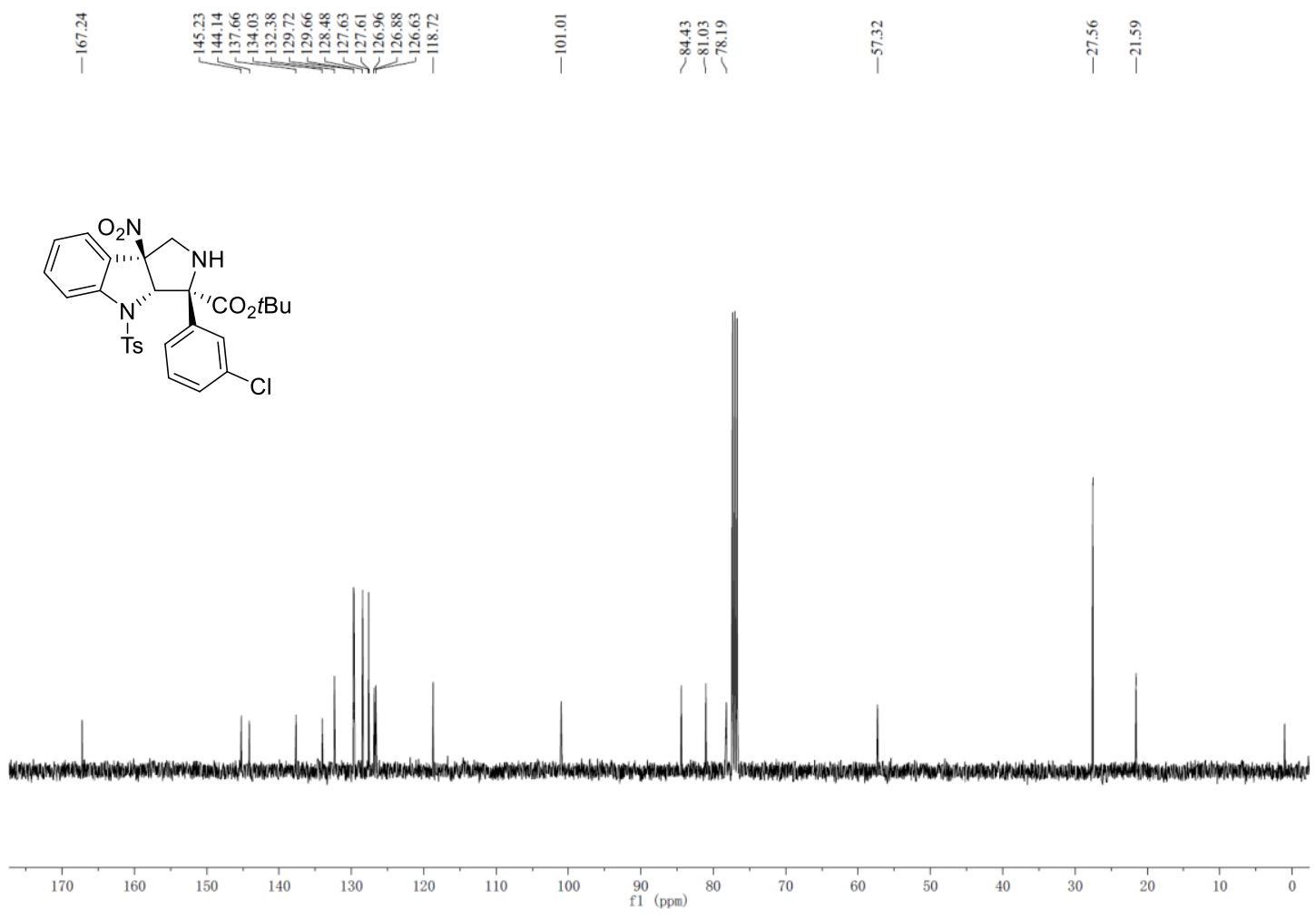
$\mathrm{mV}$

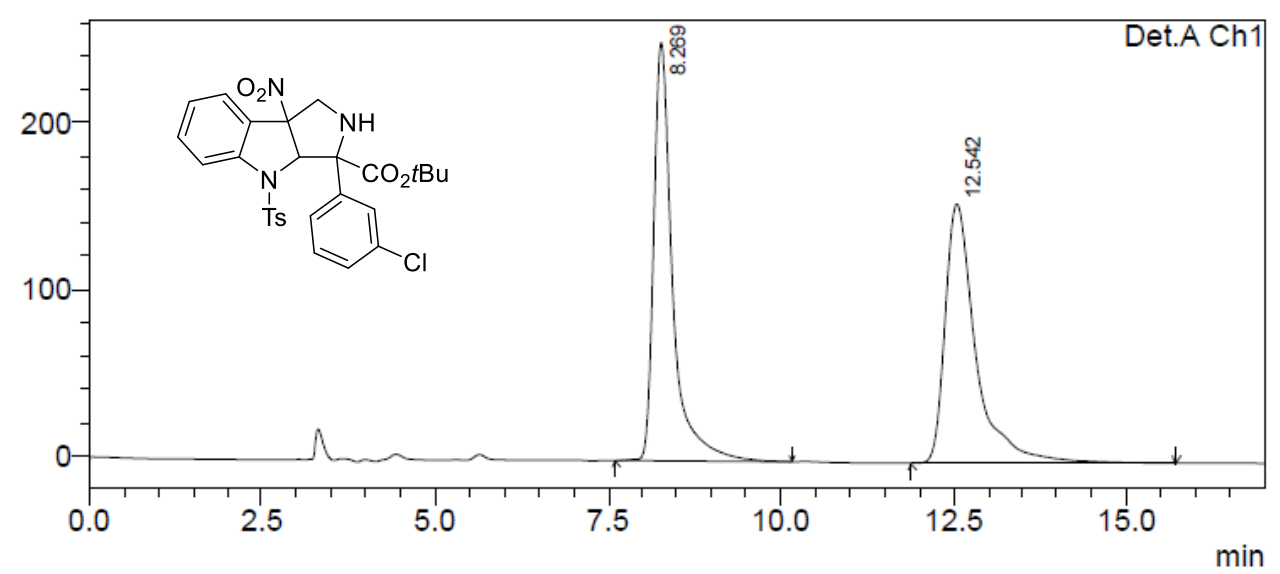

1 Det.A Ch1/254nm

PeakTable
\begin{tabular}{|c|c|c|c|c|}
\hline Petector A Ch1 $254 \mathrm{~nm}$ \\
\hline 1 & Ret. Time & Area & Height & Area $\%$ \\
\hline 2 & 8.269 & 4762520 & 250700 & 49.736 \\
\hline Total & 12.542 & 4813053 & 155253 & 50.264 \\
\hline
\end{tabular}

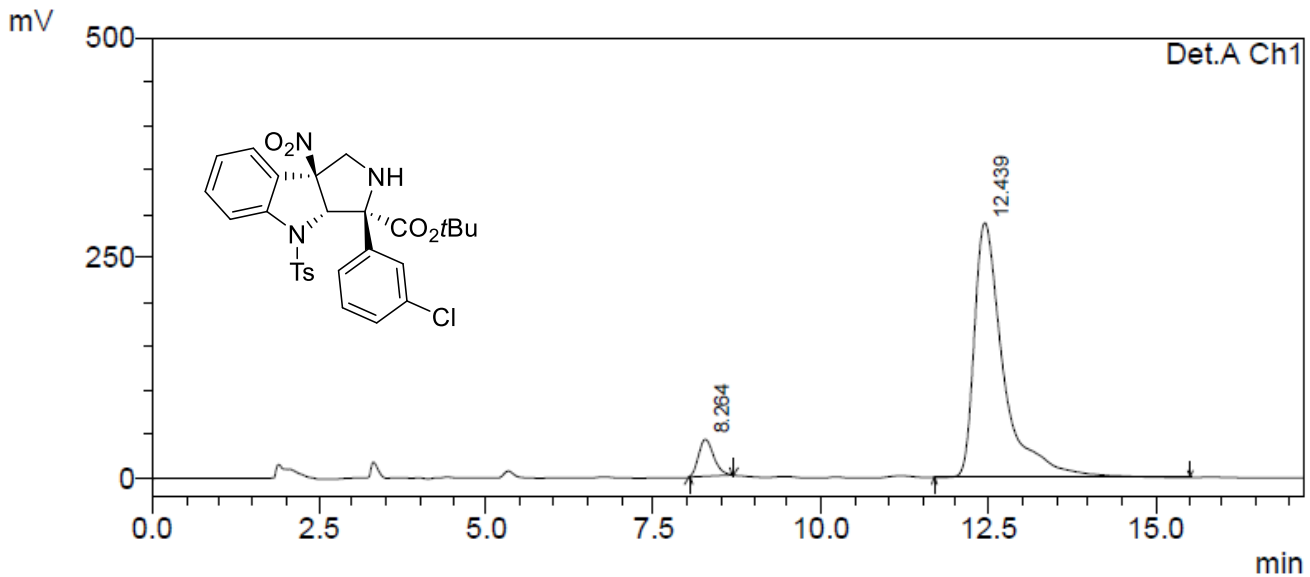

1 Det.A Ch1/254nm

PeakTable

Detector A Ch1 $254 \mathrm{~nm}$

\begin{tabular}{|c|c|c|c|c|}
\hline Peak\# & Ret. Time & Area & Height & Area \% \\
\hline 1 & 8.264 & 658732 & 41832 & 6.942 \\
\hline 2 & 12.439 & 8829894 & 288673 & 93.058 \\
\hline Total & & 9488626 & & 100.000 \\
\hline
\end{tabular}


${ }^{1} \mathrm{H}$ NMR, ${ }^{13} \mathrm{C}$ NMR and HPLC of $5 \mathrm{~h}$

(300 $\mathrm{MHz}, \mathrm{CDCl}_{3},{ }^{1} \mathrm{H}$ NMR)

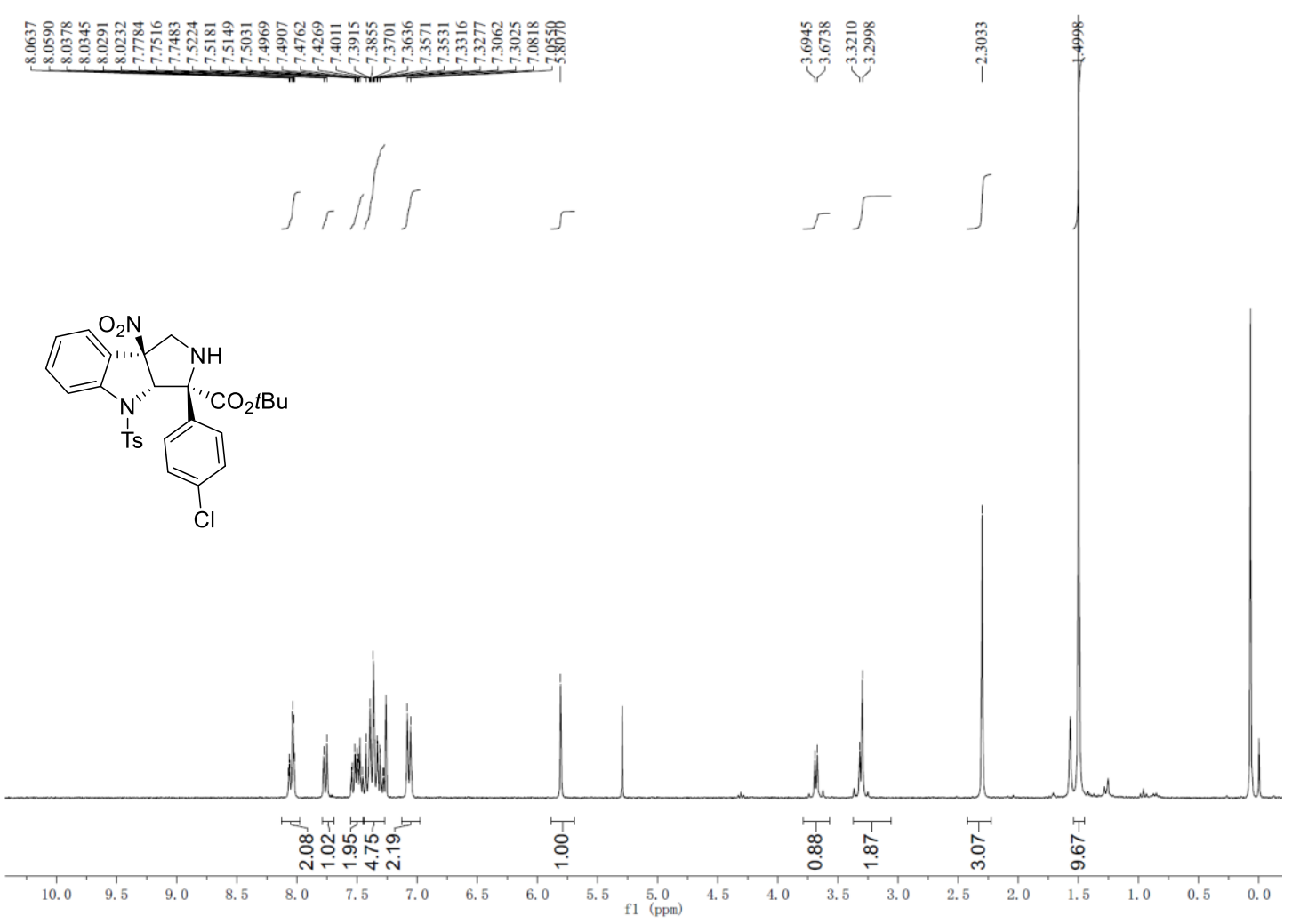

$\left(75 \mathrm{MHz}, \mathrm{CDCl}_{3},{ }^{13} \mathrm{C} \mathrm{NMR}\right)$
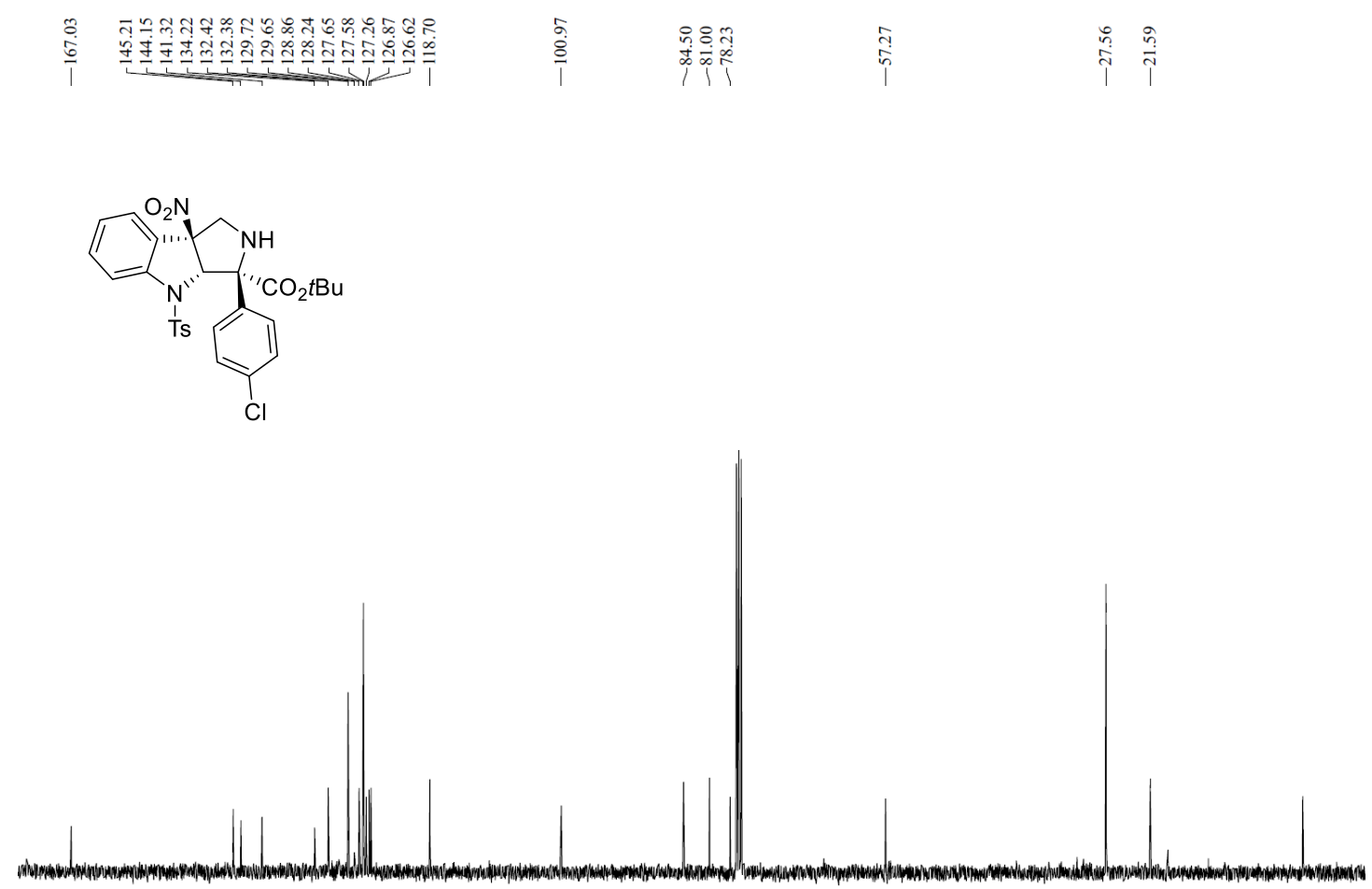

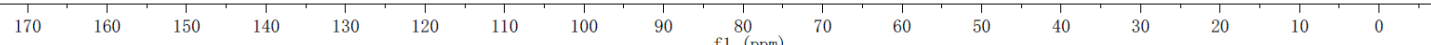


$\mathrm{mV}$

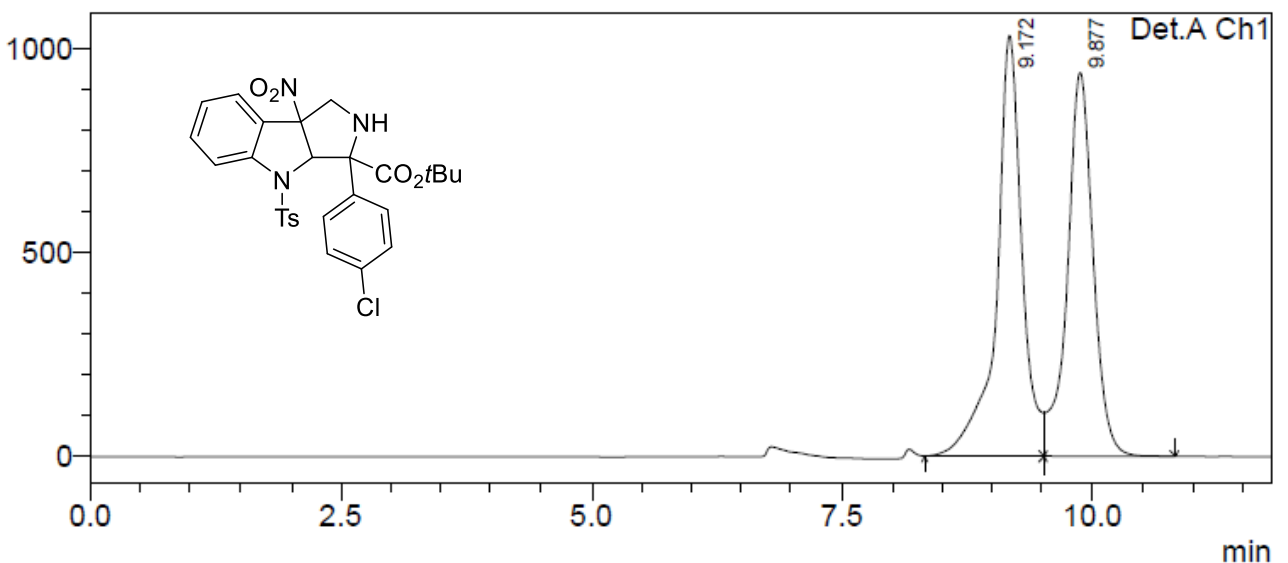

1 Det.A Ch1/254nm

Detector A Ch1 254nm

PeakTable

\begin{tabular}{|c|c|c|c|c|}
\hline Peak\# & Ret. Time & Area & Height & Area \% \\
\hline 1 & 9.172 & 18140624 & 1032595 & 51.715 \\
\hline 2 & 9.877 & 16937357 & 942151 & 48.285 \\
\hline Total & & 35077981 & & 100.000 \\
\hline
\end{tabular}

$\mathrm{m} \vee$

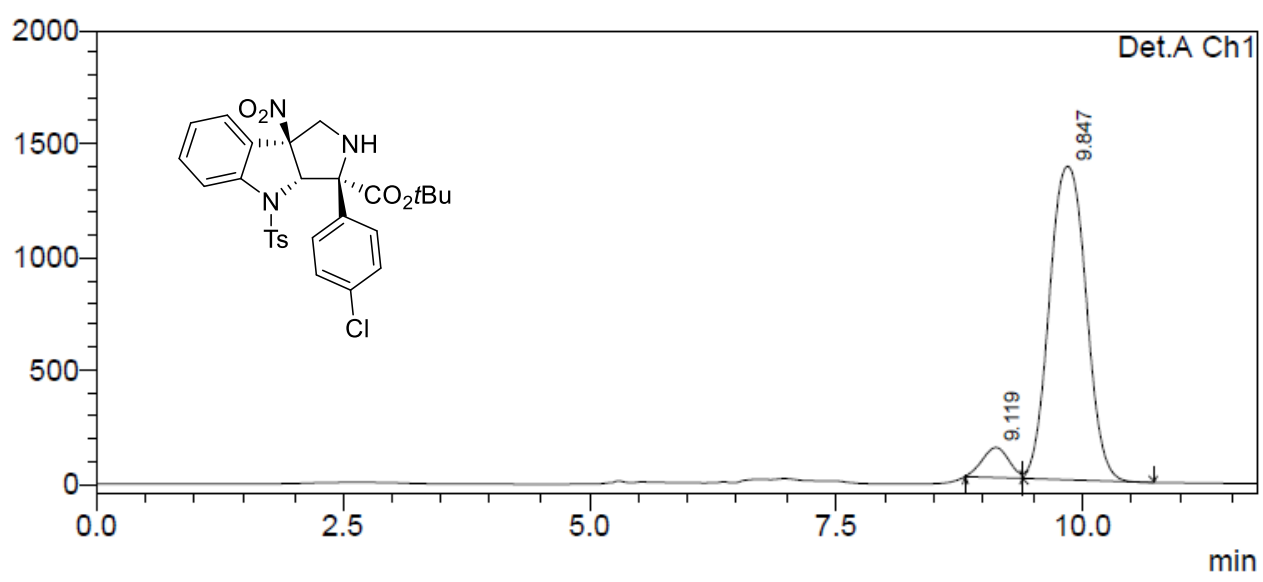

1 Det.A Ch1/254nm

PeakTable

Detector A Ch1 254nm

\begin{tabular}{|c|c|c|c|c|}
\hline Peak\# & Ret. Time & Area & Height & Area \% \\
\hline 1 & 9.119 & 2511400 & 132604 & 6.554 \\
\hline 2 & 9.847 & 35809544 & 1382042 & 93.446 \\
\hline Total & & 38320945 & & 100.000 \\
\hline
\end{tabular}


${ }^{1} \mathrm{H}$ NMR, ${ }^{13} \mathrm{C}$ NMR and HPLC of 6

(300 MHz, $\mathrm{CDCl}_{3},{ }^{1} \mathrm{H}$ NMR)

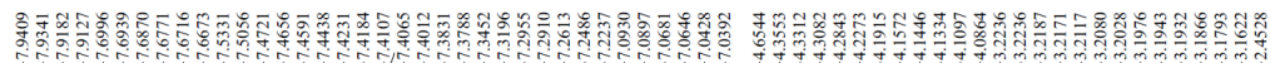

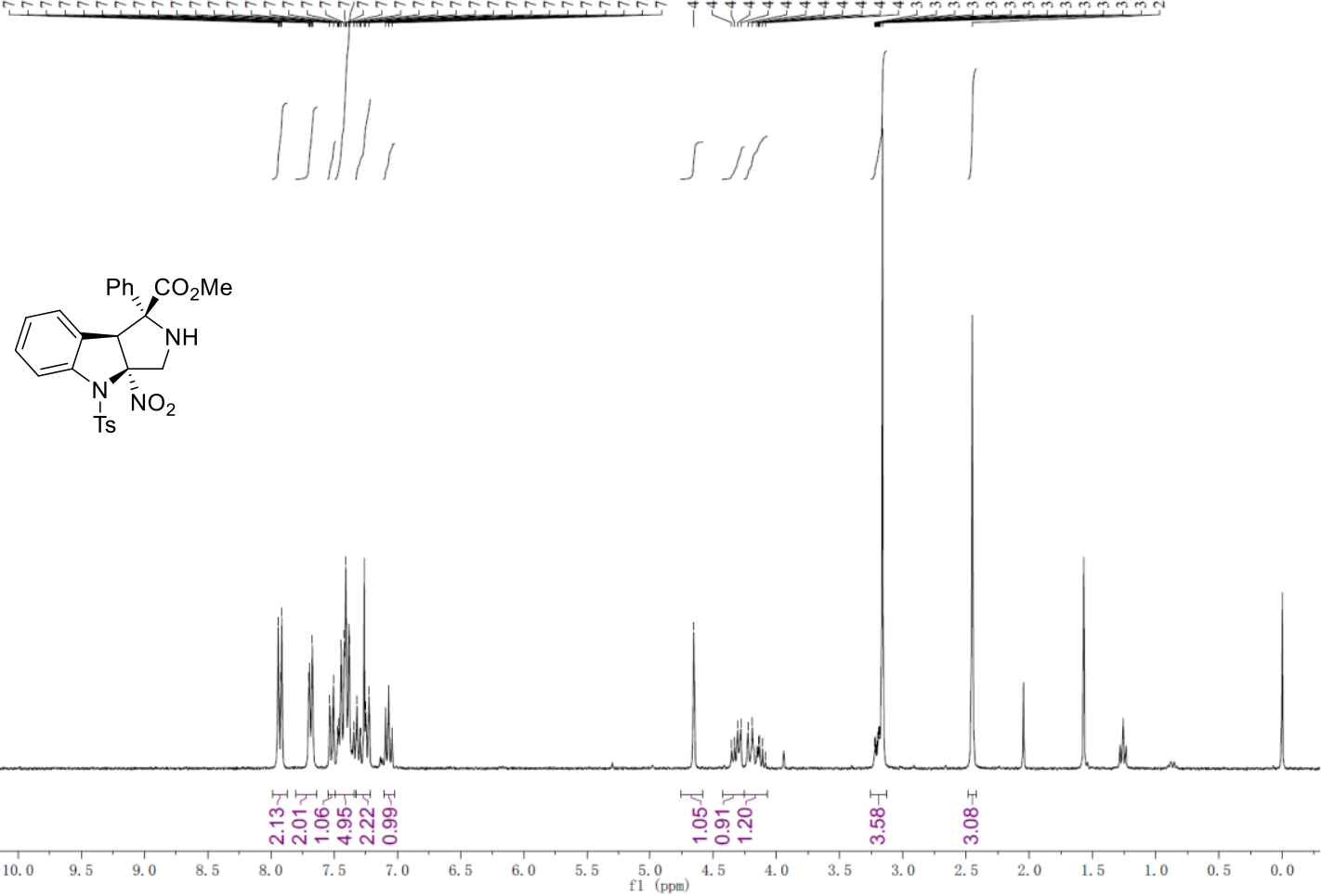

(75 $\left.\mathrm{MHz}, \mathrm{CDCl}_{3},{ }^{13} \mathrm{C} \mathrm{NMR}\right)$

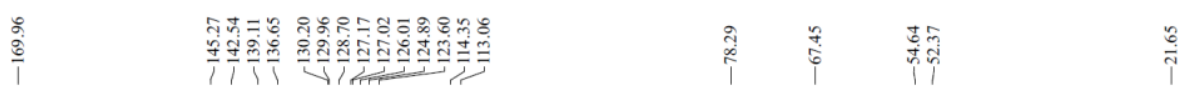
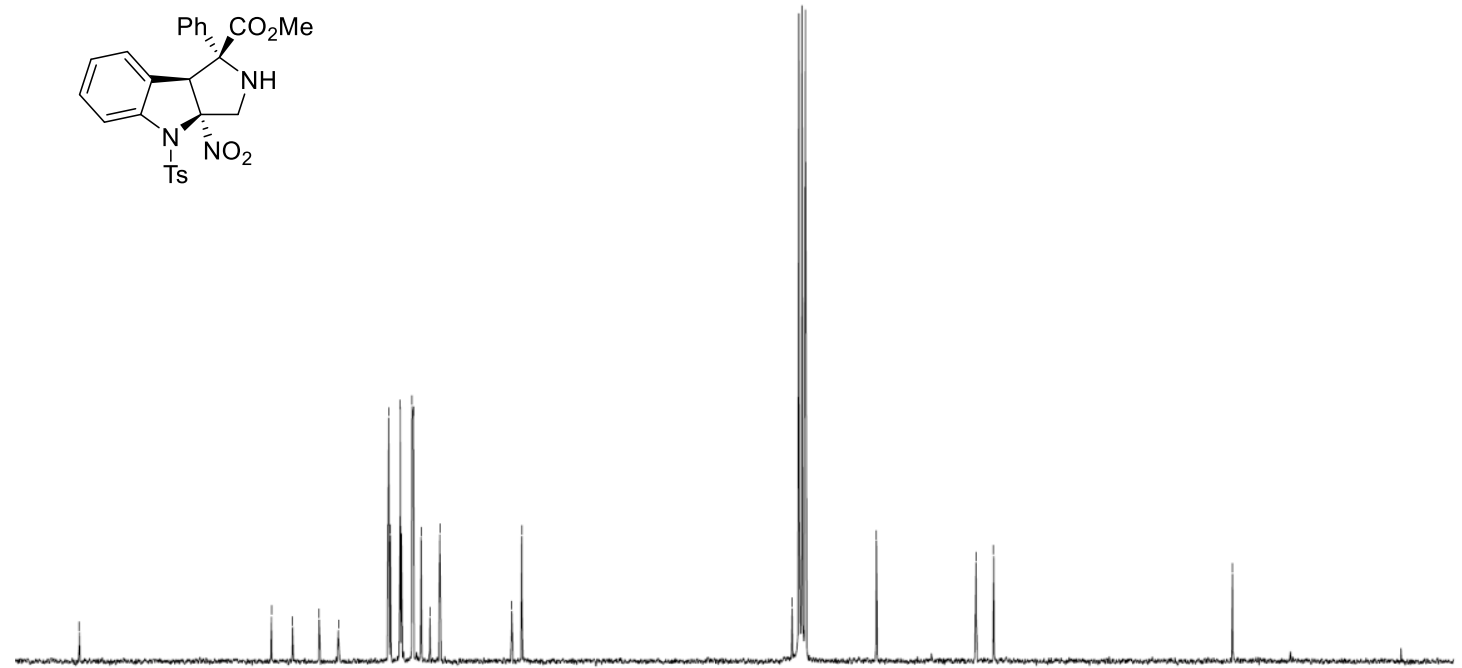
$\mathrm{mV}$

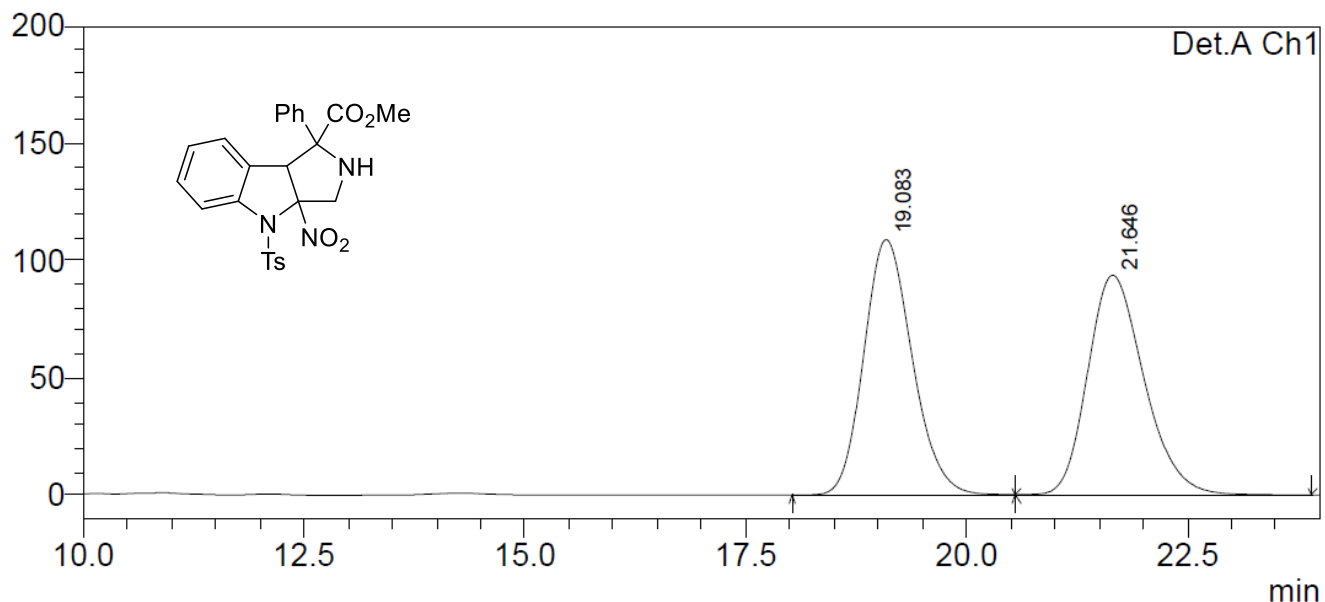

1 Det.A Ch1/254nm

Detector A Ch1 254nm

PeakTable

\begin{tabular}{|c|c|c|c|c|}
\hline Peak\# & Ret. Time & Area & Height & Area \% \\
\hline 1 & 19.083 & 4164500 & 108815 & 49.997 \\
\hline 2 & 21.646 & 4164925 & 93626 & 50.003 \\
\hline Total & & 8329425 & & 100.000 \\
\hline
\end{tabular}

$\mathrm{mV}$

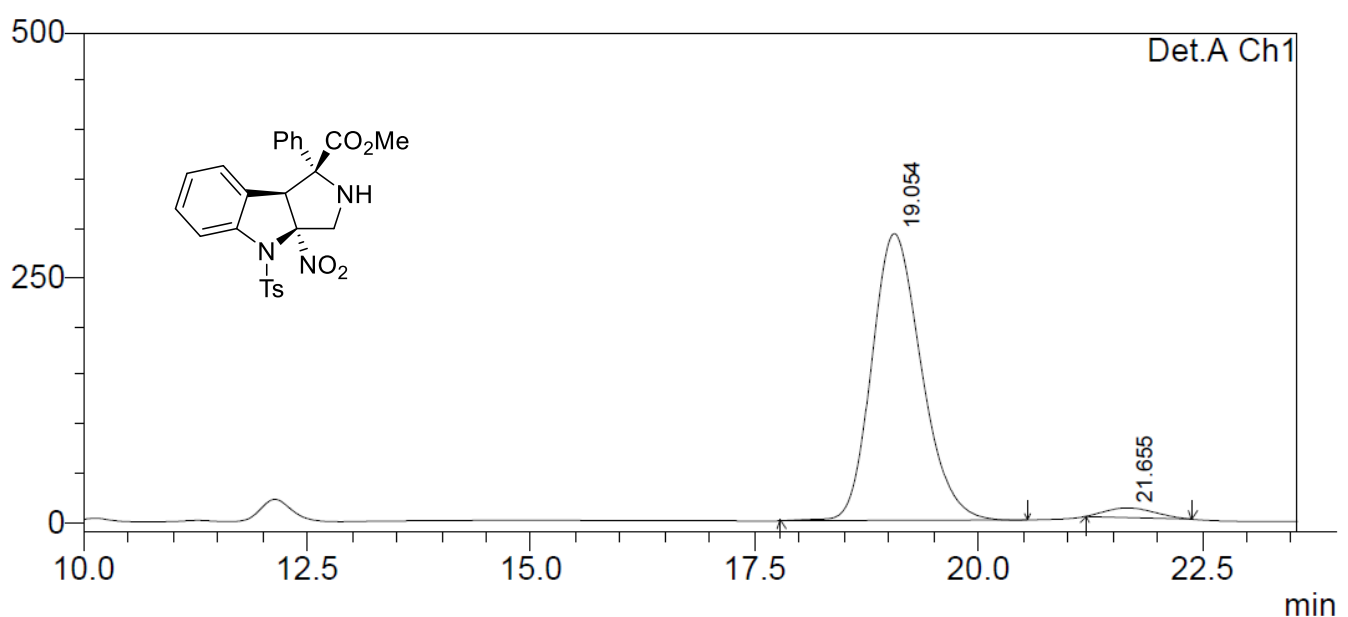

1 Det.A Ch1/254nm

PeakTable

Detector A Ch1 254nm

\begin{tabular}{|c|c|c|c|c|}
\hline Peak\# & Ret. Time & Area & Height & Area \% \\
\hline 1 & 19.054 & 11310558 & 292695 & 96.757 \\
\hline 2 & 21.655 & 379049 & 9858 & 3.243 \\
\hline Total & & 11689607 & & 100.000 \\
\hline
\end{tabular}


${ }^{1} \mathrm{H}$ NMR, ${ }^{13} \mathrm{C}$ NMR and HPLC of 7

(300 $\mathrm{MHz}, \mathrm{CDCl}_{3},{ }^{1} \mathrm{H}$ NMR)
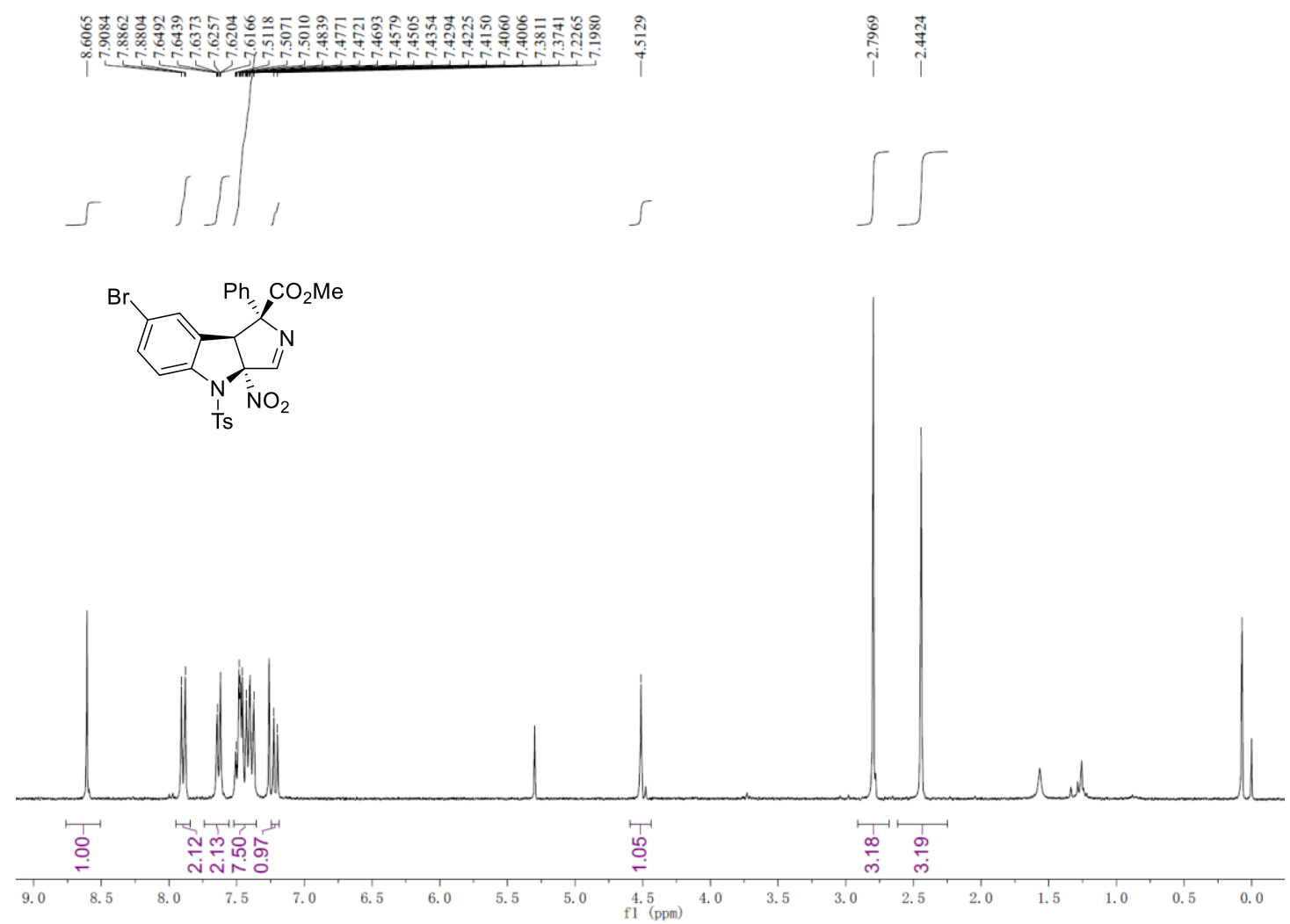

(75 $\mathrm{MHz}, \mathrm{CDCl}_{3},{ }^{13} \mathrm{C}$ NMR)

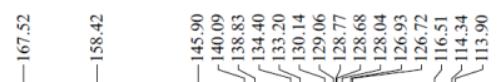
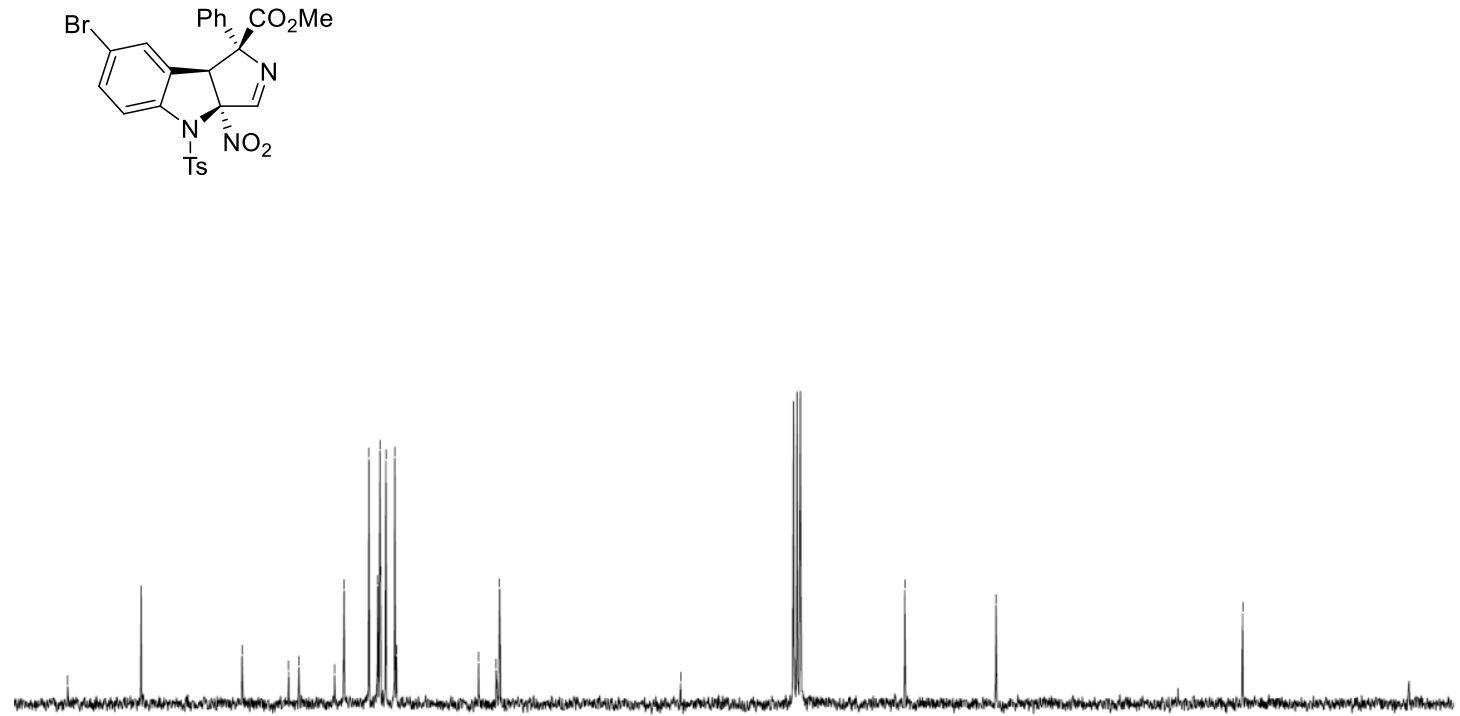

$\begin{array}{lllllllll}170 & 160 & 150 & 140 & 130 & 120 & 110 & 100 & 90 \\ & & & & & & & 80(\mathrm{ppm})\end{array}$ 
$\mathrm{mV}$

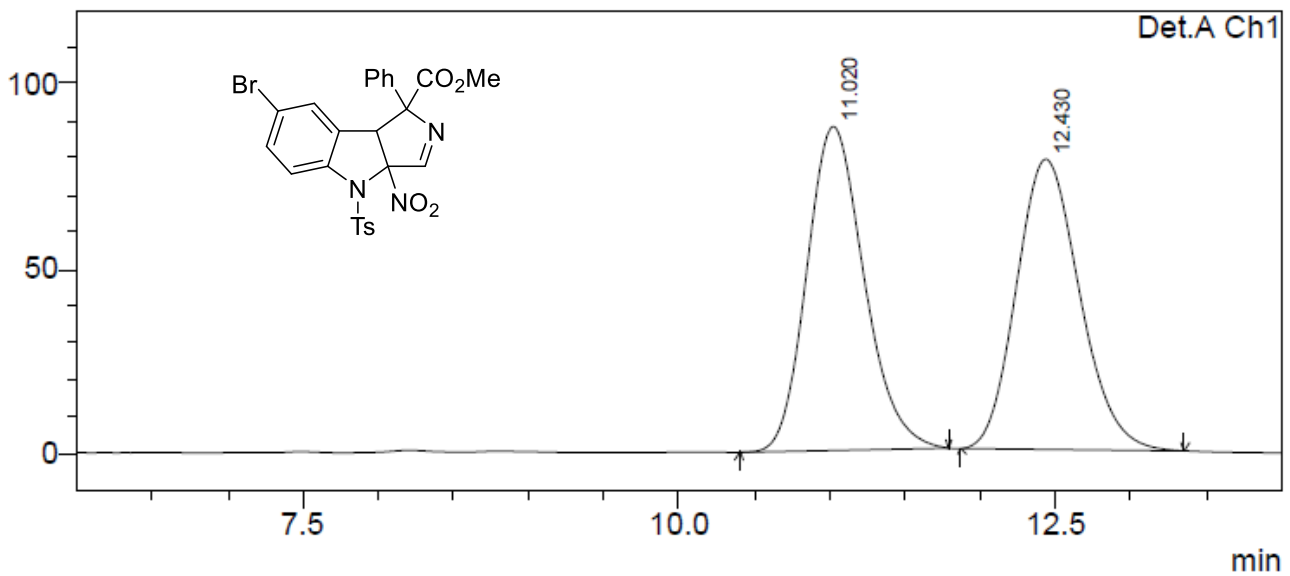

1 Det.A Ch1/254nm

PeakTable

Detector A Ch1 254nm

\begin{tabular}{|c|c|c|c|c|}
\hline Peak\# & Ret. Time & Area & Height & Area \% \\
\hline 1 & 11.020 & 2319178 & 87644 & 50.287 \\
\hline 2 & 12.430 & 2292668 & 78554 & 49.713 \\
\hline Total & & 4611846 & & 100.000 \\
\hline
\end{tabular}

$\mathrm{mV}$

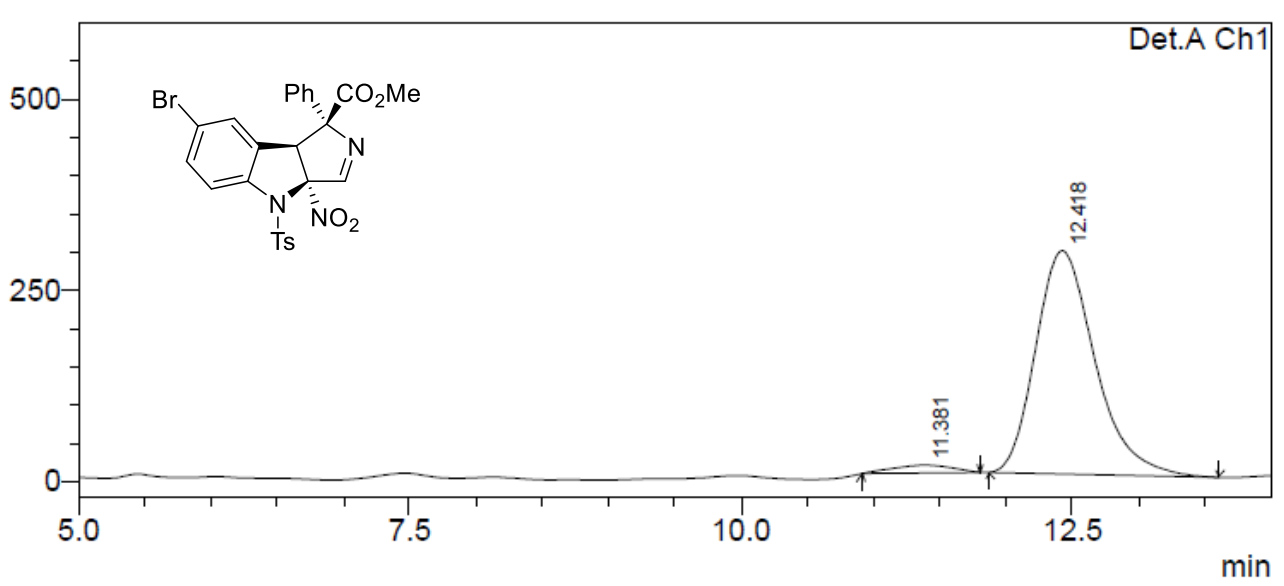

1 Det.A Ch1/254nm

PeakTable

Detector A Ch1 254nm

\begin{tabular}{|c|c|c|c|c|}
\hline Peak\# & Ret. Time & Area & Height & Area \% \\
\hline 1 & 11.381 & 318749 & 10203 & 3.392 \\
\hline 2 & 12.418 & 9078893 & 291250 & 96.608 \\
\hline Total & & 9397643 & & 100.000 \\
\hline
\end{tabular}


${ }^{1} \mathrm{H}$ NMR, ${ }^{13} \mathrm{C}$ NMR and HPLC of 8

(300 $\left.\mathrm{MHz}, \mathrm{CDCl}_{3},{ }^{1} \mathrm{H} \mathrm{NMR}\right)$

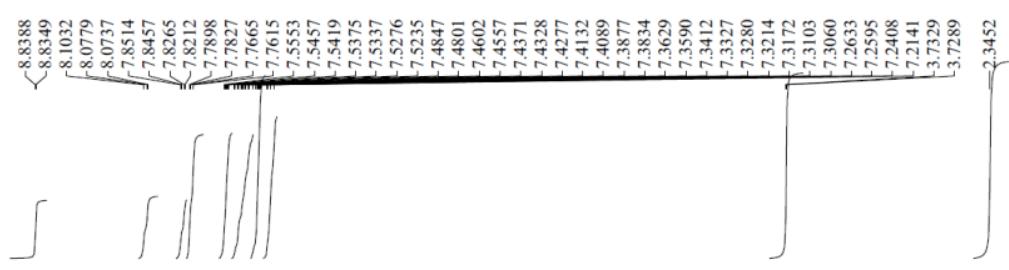

(1)

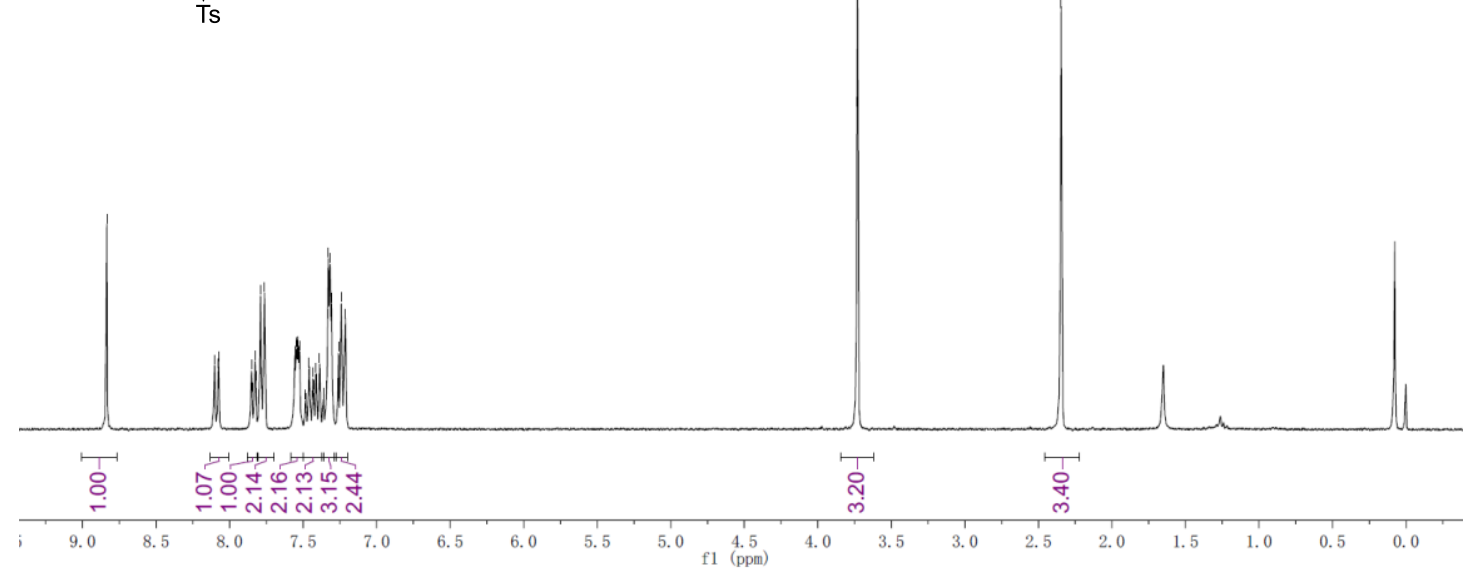

(75 MHz, $\mathrm{CDCl}_{3},{ }^{13} \mathrm{C}$ NMR)
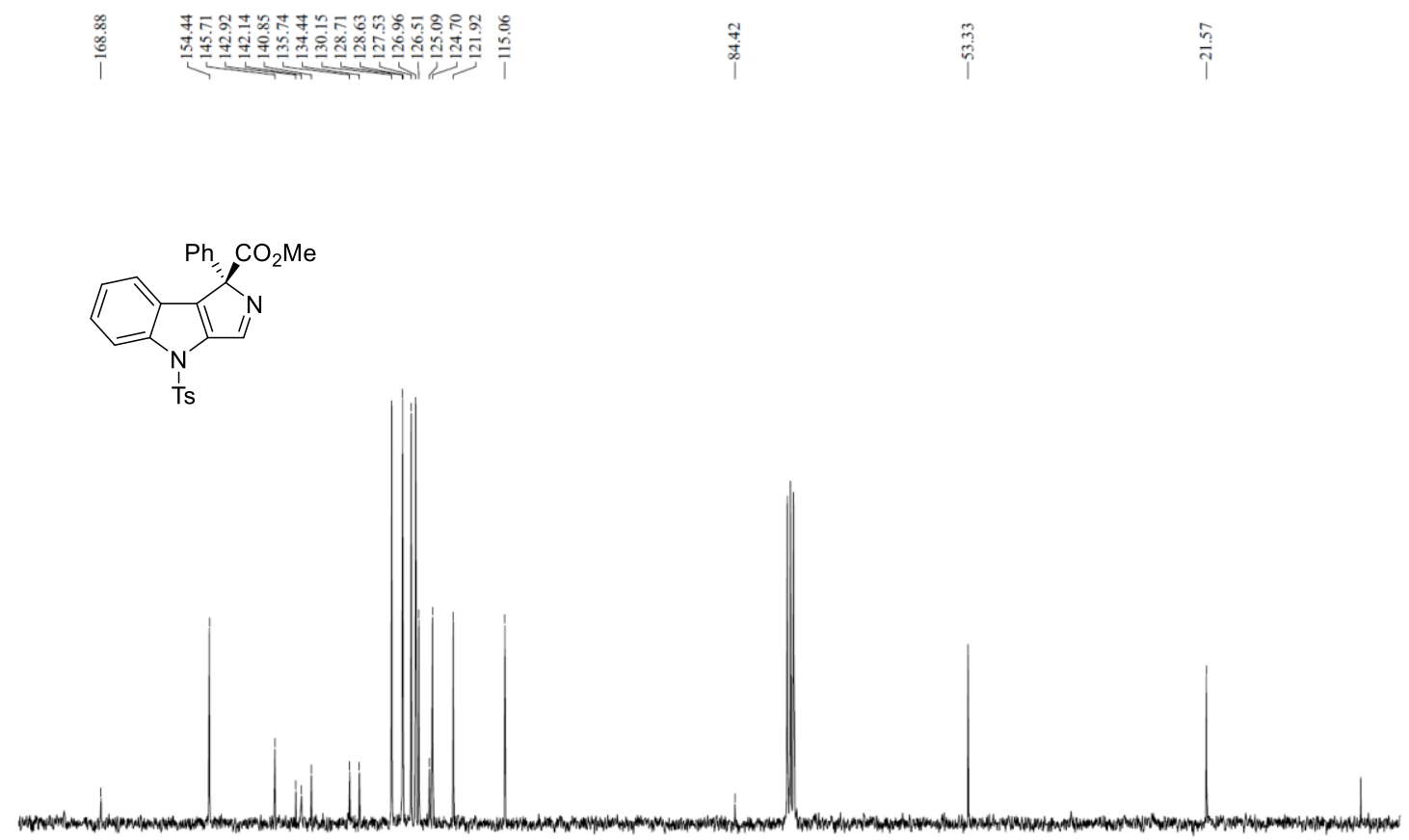

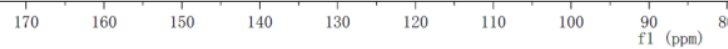


$\mathrm{mV}$

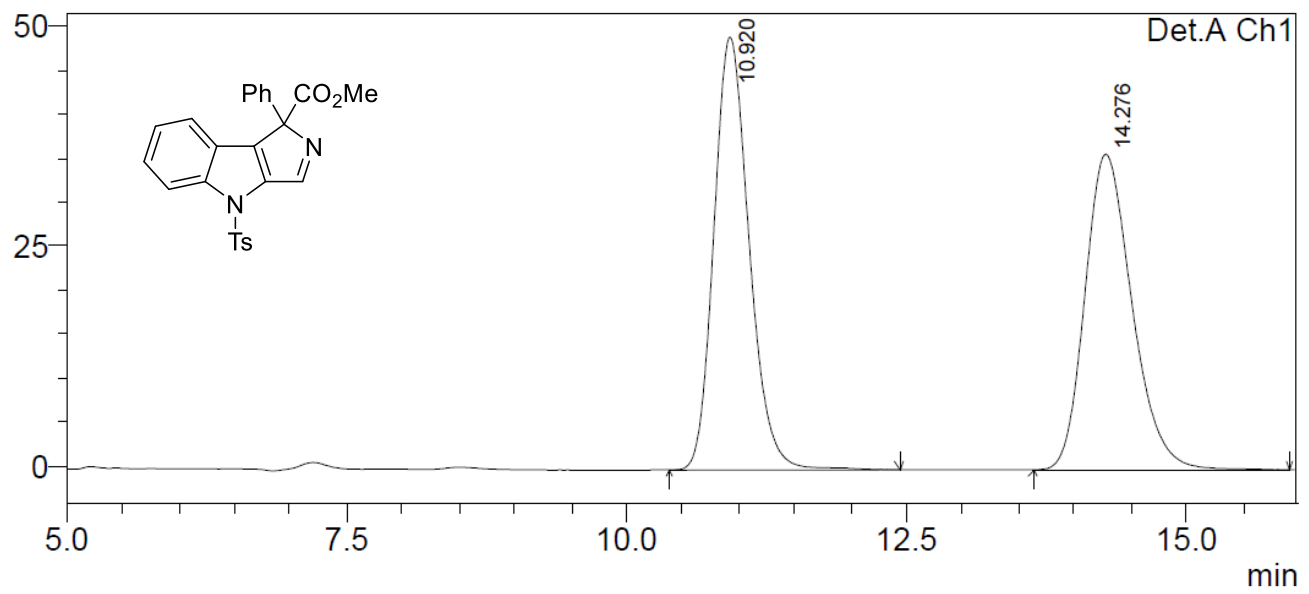

1 Det.A Ch1/254nm

PeakTable

Detector A Ch1 254nm

\begin{tabular}{|c|c|c|c|c|}
\hline Peak\# & Ret. Time & Area & Height & Area \% \\
\hline 1 & 10.920 & 1078303 & 49109 & 50.694 \\
\hline 2 & 14.276 & 1048789 & 35815 & 49.306 \\
\hline Total & & 2127093 & & 100.000 \\
\hline
\end{tabular}

$\mathrm{mV}$

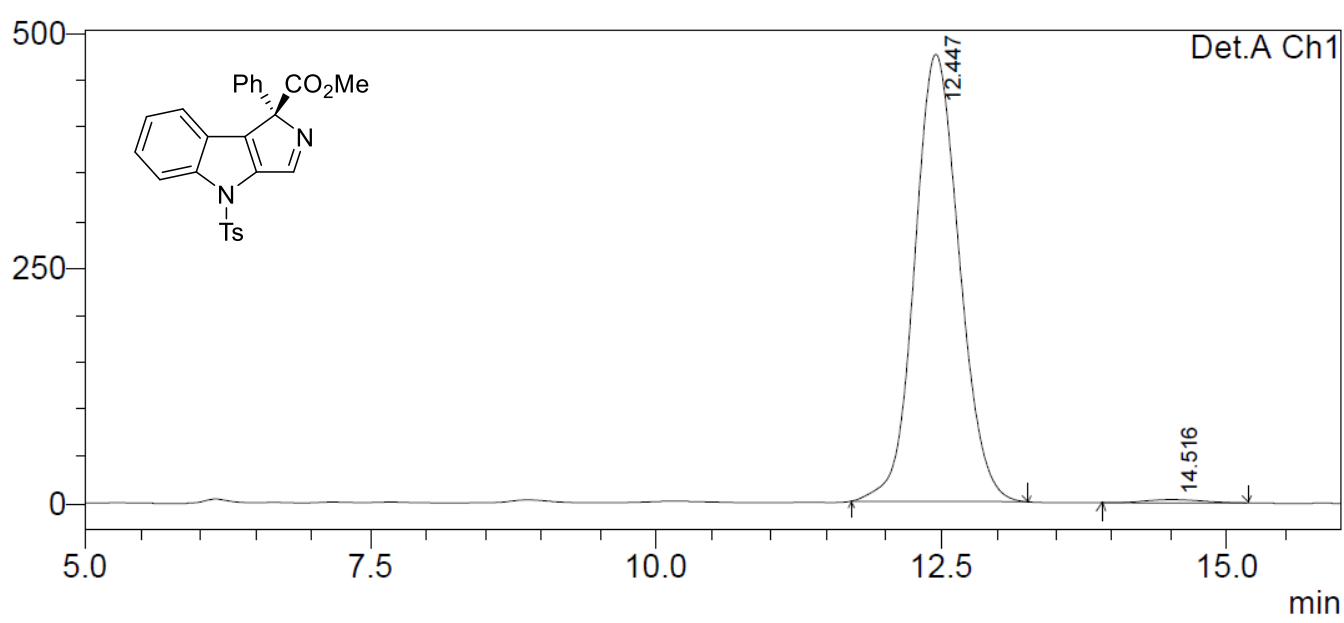

1 Det.A Ch $1 / 254 \mathrm{~nm}$

PeakTable

Detector A Ch1 254nm

\begin{tabular}{|c|c|c|c|c|}
\hline Peak\# & Ret. Time & Area & Height & Area \% \\
\hline 1 & 12.447 & 12955266 & 475187 & 99.065 \\
\hline 2 & 14.516 & 122211 & 3631 & 0.935 \\
\hline Total & & 13077477 & & 100.000 \\
\hline
\end{tabular}


${ }^{1} \mathrm{H}$ NMR, ${ }^{13} \mathrm{C}$ NMR and HPLC of 9

(300 $\mathrm{MHz}, \mathrm{CDCl}_{3},{ }^{1} \mathrm{H}$ NMR)

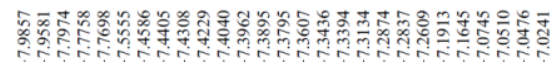

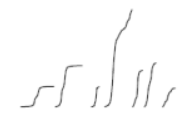

Ts

(75 $\mathrm{MHz}, \mathrm{CDCl}_{3},{ }^{13} \mathrm{C}$ NMR)

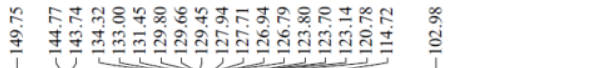

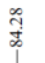

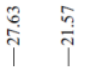
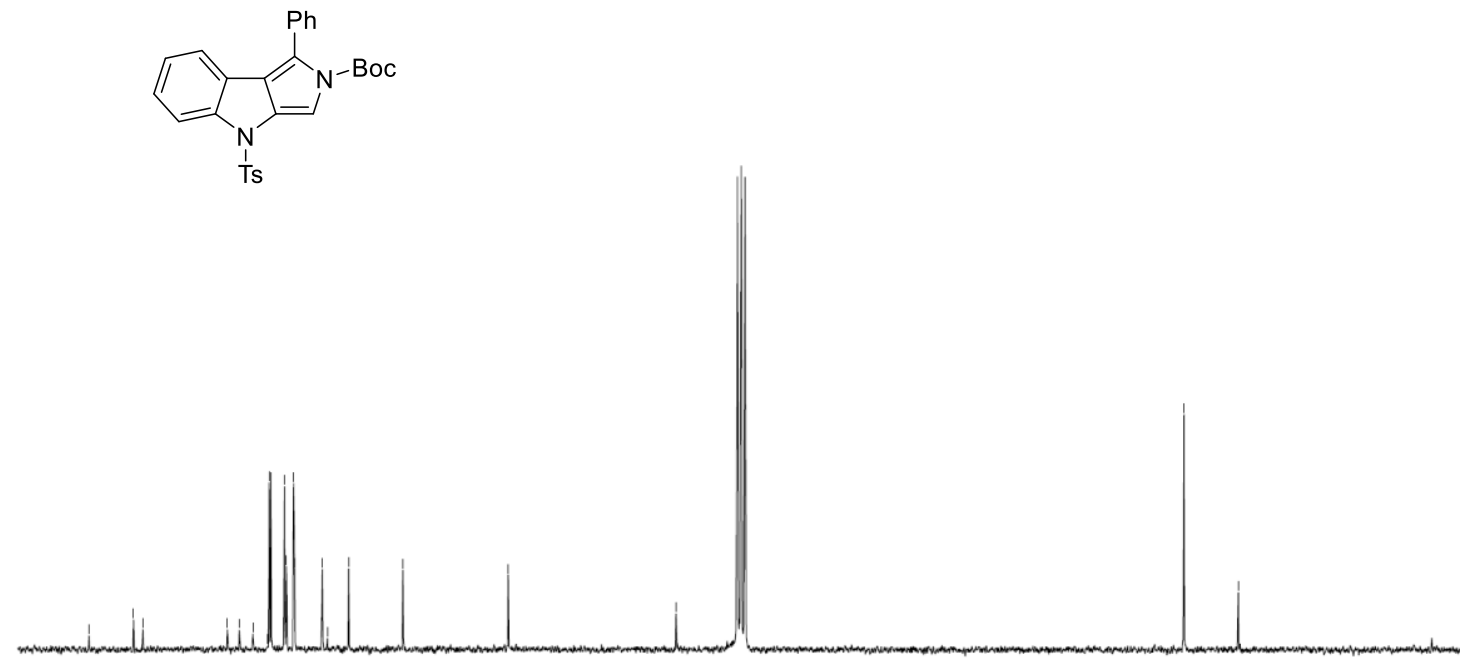

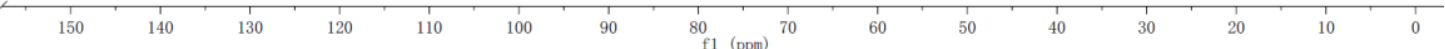


${ }^{1} \mathrm{H}$ NMR, ${ }^{13} \mathrm{C}$ NMR and HPLC of 10

(300 $\mathrm{MHz}, \mathrm{CDCl}_{3},{ }^{1} \mathrm{H}$ NMR)

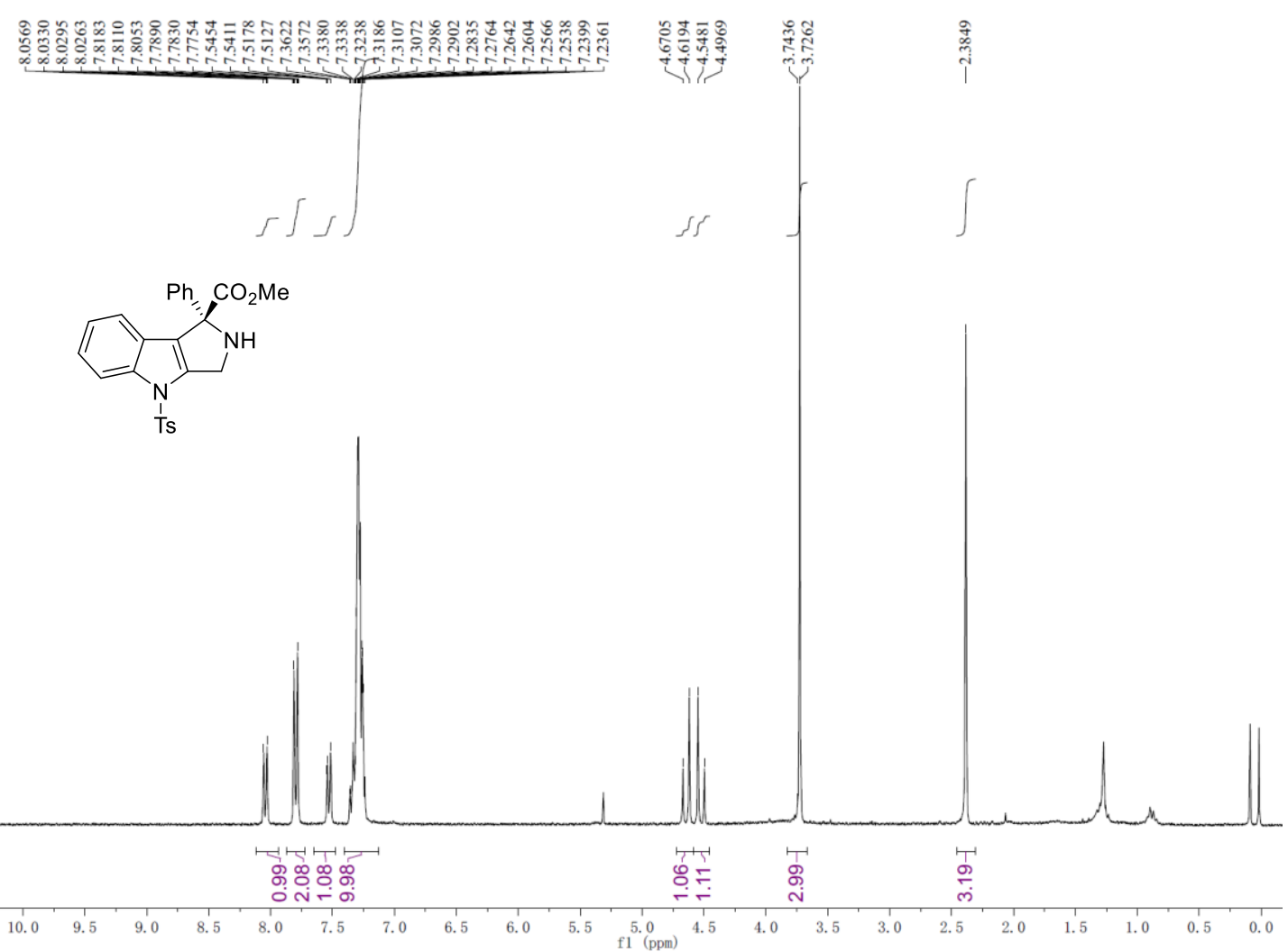

(75 $\left.\mathrm{MHz}, \mathrm{CDCl}_{3},{ }^{13} \mathrm{C} \mathrm{NMR}\right)$

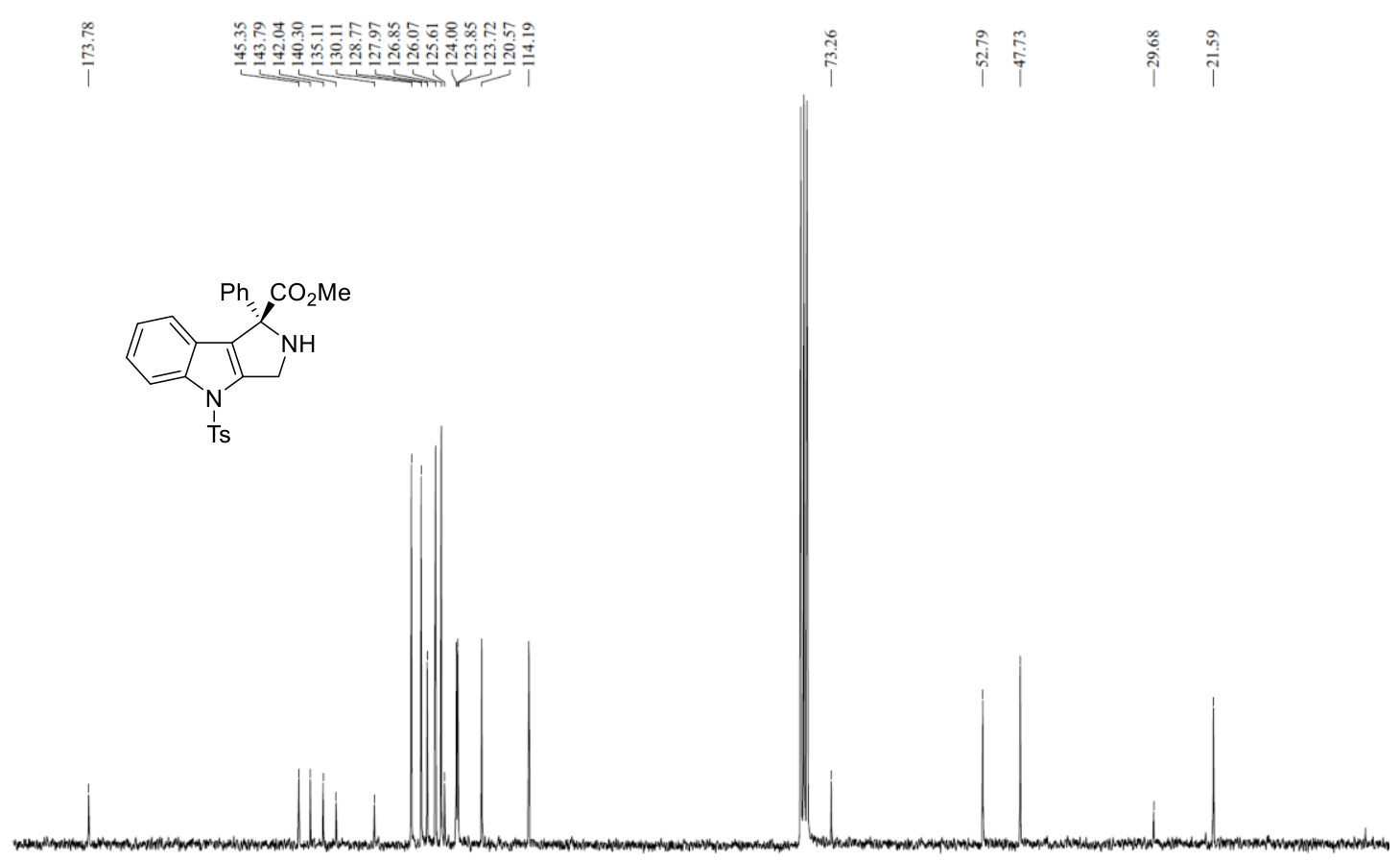

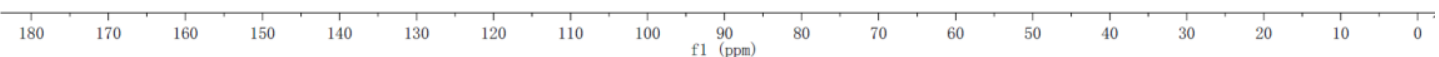


$\mathrm{mv}$

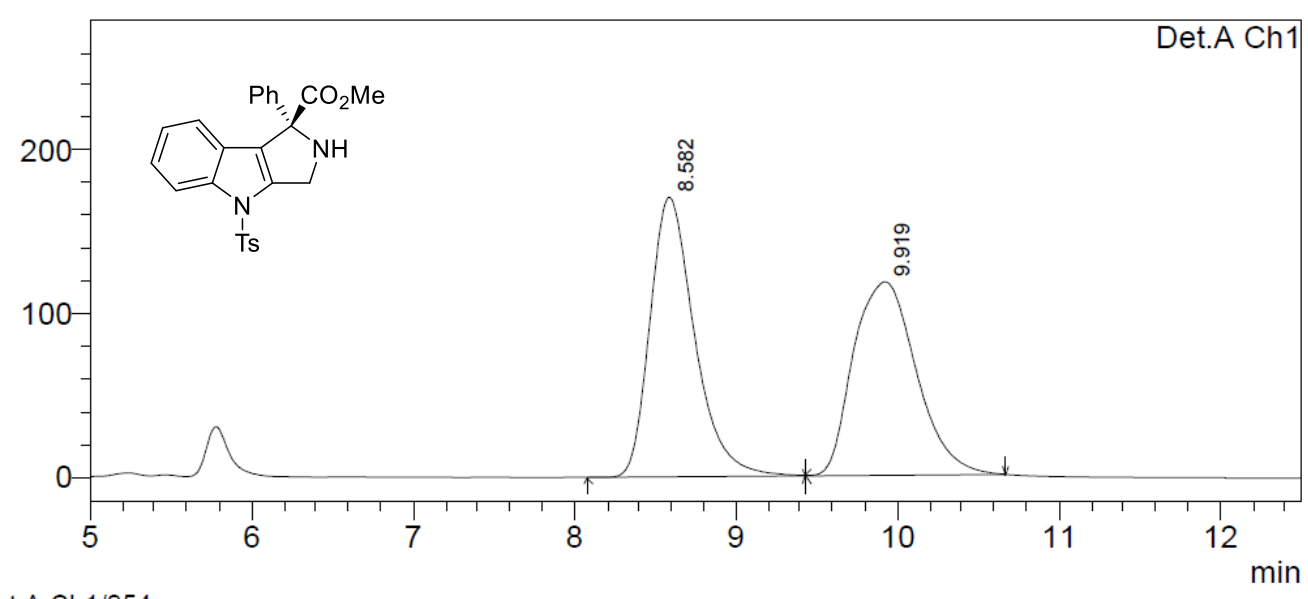

1 Det.A Ch1/254nm

PeakTable

Detector A Ch1 254nm

\begin{tabular}{|c|c|c|c|c|}
\hline Peak\# & Ret. Time & Area & Height & Area \% \\
\hline 1 & 8.582 & 3277667 & 170945 & 50.513 \\
\hline 2 & 9.919 & 3211076 & 118320 & 49.487 \\
\hline Total & & 6488744 & & 100.000 \\
\hline
\end{tabular}

$\mathrm{mV}$

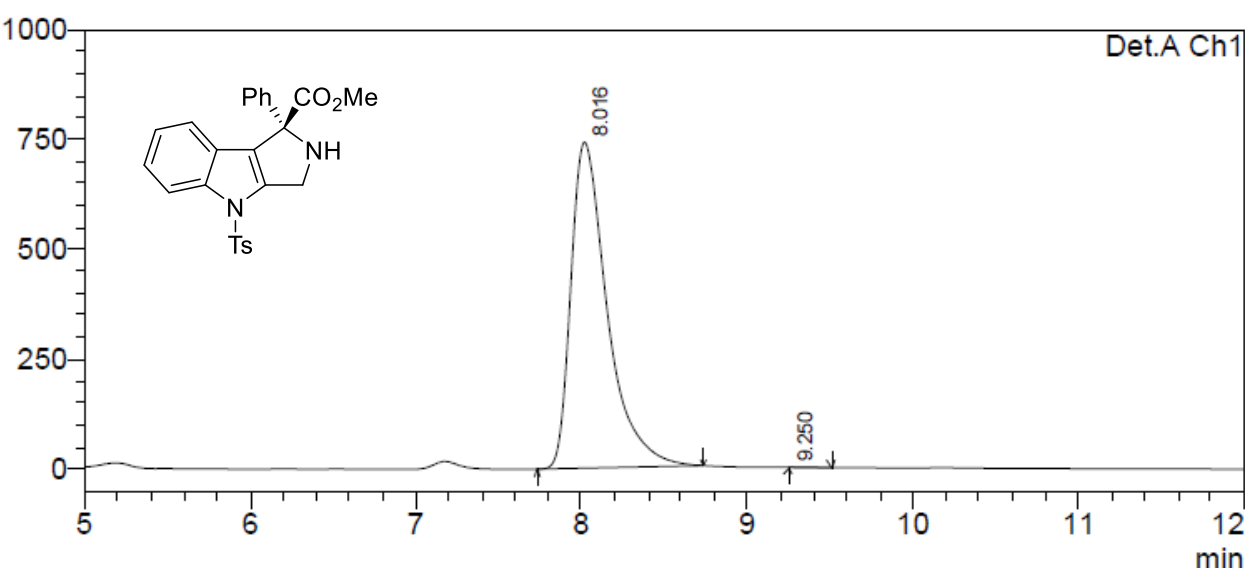

1 Det.A Ch1/254nm

PeakTable

Detector A Ch1 $254 \mathrm{~nm}$
\begin{tabular}{|c|c|c|c|c|}
\hline Peak\# & Ret. Time & Area & Height & Area $\%$ \\
\hline 1 & 8.016 & 11260902 & 740746 & 99.996 \\
\hline 2 & 9.250 & 473 & 0 & 0.004 \\
\hline Total & & 11261375 & & 100.000 \\
\hline
\end{tabular}

